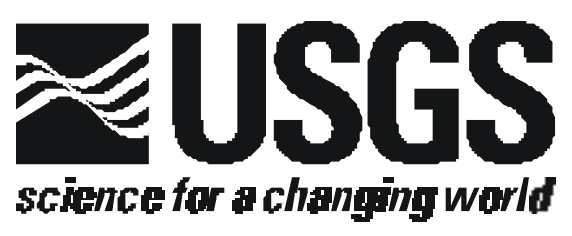

\title{
MAGNETOTELLURIC DATA ACROSS THE BATTLE MOUNTAIN-EUREKA AND CARLIN TRENDS, NEAR THE $41^{\text {ST }}$ PARALLEL, NEVADA
}

\author{
by \\ Jackie M. Williams \\ and \\ Brian D. Rodriguez 1
}

Open-File Report 01-278

on-line edition

2001

This report is preliminary and has not been reviewed for conformity with U.S. Geological Survey editorial standards and stratigraphic nomenclature. Any use of trade, product, or firm names is for descriptive purposes only and does not imply endorsement by the U.S.

Government.

U.S. DEPARTMENT OF THE INTERIOR

U.S. GEOLOGICAL SURVEY

1 Denver, Colorado 
TABLE OF CONTENTS

INTRODUCTION

MAGNETOTELLURIC METHOD 3

MAGNETOTELLURIC SURVEY

MAGNETOTELLURIC DATA

REFERENCES CITED 9

APPENDIX - MAGNETOTELLURIC DATA PLOTS 12 


\section{INTRODUCTION}

Many sediment-hosted gold deposits occur along linear trends in northern Nevada. The distribution and genesis of these deposits along the Battle Mountain-Eureka and Carlin gold trends is not fully understood. In general, most models agree that regional structures played an important role in the spatial distribution of these deposits (e.g. Arehart and others, 1993; Ilchik and Barton, 1997; Radtke, 1985; Shawe, 1991; Sillitoe and Bonham, 1990; Tosdal, 1998). To investigate crustal structures that may be related to the genesis of gold deposits along these trends, a regional westeast profile of magnetotelluric (MT) soundings was acquired in 1998 and 2000 (line MT5-MT5', Figure 1). Resistivity modeling of the MT data can be used to infer the deep resistivity structure of the crust to investigate possible tectonic controls on the emplacement of mineral deposits along these linear trends that may be used to help improve critical gold endowment estimates in the Humboldt River Basin. The purpose of this report is to release the MT sounding data; no interpretation of the data is included.

\section{MAGNETOTELLURIC METHOD}

The magnetotelluric (MT) method is a passive surface geophysical technique, which uses the earth's natural electromagnetic fields to investigate the electrical resistivity structure of the subsurface. The resistivity of geologic units is largely dependent upon their fluid content, porosity, degree of fracturing, temperature, and conductive mineral content (Keller, 1989). Saline fluids within the pore spaces and fracture openings can reduce resistivities in a resistive rock matrix. Also, resistivity can be lowered by the presence of conductive clay minerals, carbon, and metallic mineralization. It is common for altered volcanic rocks to contain authigenic minerals that have resistivities ten times lower than those of the surrounding rocks (Nelson and Anderson, 1992). Increased temperatures cause higher ionic mobility and mineral activation energy, reducing rock resistivities significantly. Unaltered, unfractured igneous rocks are normally very resistive (typically 1,000 ohm-m or greater), whereas fault zones will show low resistivity (less than $100 \mathrm{ohm}-\mathrm{m}$ ) when they are comprised of rocks fractured enough to have hosted fluid transport and consequent mineralogical alteration (Eberhart-Phillips and others, 1995). Carbonate rocks are moderately to highly resistive (hundreds to thousands of ohm-m) depending upon their fluid content, porosity, fracturing, and impurities. Marine shales, mudstones, and clay-rich alluvium are normally very conductive 
(a few ohm-m to tens of ohm-m). Unaltered, metamorphic rocks (non-graphitic) are moderately to highly resistive (hundreds to thousands of ohm-m). Tables of electrical resistivity for a variety of rocks, minerals and geological environments may be found in Keller (1987) and Palacky (1987).

The MT method can be used to probe the crust from depths of tens of meters to depths of tens of kilometers (Vozoff, 1991). Natural variations of the Earth's magnetic and electric field are measured and recorded at each MT station. The primary frequency bands used by the MT method are 10,000 $\mathrm{Hz}$ to $1 \mathrm{~Hz}$ from worldwide lightning activity and $1 \mathrm{~Hz}$ to $0.0001 \mathrm{~Hz}$ from geomagnetic micro-pulsations. Natural electric and magnetic fields propagate vertically in the earth because the very large resistivity contrast between the air and the earth causes a vertical refraction of both fields transmitted into the earth (Vozoff, 1972).

The natural electric and magnetic fields are recorded in two orthogonal, horizontal directions. The vertical magnetic field ("tipper") is also recorded. The resulting time-series signals are used to derive the tensor apparent resistivity and phase. First the signals are converted to complex crossspectra using FFT (fast-Fourier-transform) techniques. Then, least-squares, cross-spectral analysis (Bendat and Piersol, 1971) is used to solve for a transfer function that relates the observed electric fields to the magnetic fields under the assumption that the Earth consists of a two-input, two-output, linear system with the magnetic fields as input and the electric fields as output (Rodriguez and others, 1996). Prior to conversion to apparent resistivity and phase, the tensor is normally rotated into principal directions that correspond to the direction of maximum and minimum apparent resistivity. For a two-dimensional (2-D) Earth, the MT fields can be decoupled into transverse electric (TE) and transverse magnetic (TM) modes; 2-D modeling is generally done to fit both modes. When the geology satisfies the 2-D assumption, the MT data for the TE mode is for the electric field parallel to geologic strike, and the data for the TM mode is for the electric field across strike. The MT method is well suited for studying complicated geological environments because the electric and magnetic relations are sensitive to vertical and horizontal variations in resistivity. The method is capable of establishing whether the electromagnetic fields are responding to subsurface terranes of effectively 1-, 2-, or 3-dimensions. An introduction to the MT method and references for a more advanced understanding are contained in Dobrin and Savit (1988) and Vozoff (1991). 


\section{MAGNETOTELLURIC SURVEY}

Seventeen MT soundings were located along or near profile MT5-MT5" (Figure 1) with spacing that varied from 1.5 to 13.0 kilometers. The profile is oblique to the Battle MountainEureka and Carlin trends. All stations were collected with a portable EMI MT-1 system (EMI, 1996). Horizontal electric fields were sensed using an L-shaped, three-electrode array with dipole lengths of 30 meters. The orthogonal, horizontal magnetic fields in the direction of the electric-field measurement array were sensed using permalloy-cored induction coils (Stanley and Tinkler, 1983). The vertical magnetic field was recorded only at stations 53, 48, 59, and 37 . Frequencies sampled ranged from 100 to $0.004 \mathrm{~Hz}$ using single station recordings of both orthogonal horizontal components of the electric and magnetic fields. Sampling this frequency range in previous areas of widely varying geology has allowed us to probe the crust from depths of hundreds of meters to depths of tens of kilometers.

The recorded time-series data were transformed to the frequency domain and processed to determine a two-dimensional apparent resistivity and phase tensor at each site. Rotation of the impedance tensor to maximum and minimum directions allows for decoupling into the $\mathrm{TE}$ and $\mathrm{TM}$ modes. Local reference sensors to help reduce bias in the impedance determinations due to instrument or environmental noise (Gamble and others, 1979a; Clarke and others, 1983) were used at all stations except station 37. Although true remote reference techniques were not used in our survey, we did sort cross-power files to select optimal signal-to-noise data sets (see Appendix).

The effects of near-surface resistivity anomalies cause "static shifts" (Sternberg and others, 1988) in the data. Static shifts of this data set ranged from 0.0 to 1.5 of a $10 \mathrm{~g}$ decade. Only stations 61, 57, and 32 had static shifts of 0.4 of a log decade or greater. The remainder of the stations had an average of 0.1 of a log decade static shift.

\section{MAGNETOTELLURIC DATA}

The following table shows seventeen magnetotelluric (MT) station locations (from southwest to northeast). Coordinates are referenced to the 1866 Clarke spheroid and North American 1927 Western United States datum. Longitude and latitude format below is decimal degrees. Elevation is in meters. 


\begin{tabular}{|c|c|c|c|}
\hline Station & Longitude & Latitude & Elev (m) \\
\hline 53 & $\overline{-117.46039}$ & $\overline{41.03561}$ & 1450 \\
\hline 61 & -117.37602 & 41.05213 & 1430 \\
\hline 48 & -117.23083 & 41.04530 & 1340 \\
\hline 62 & -117.12730 & 41.03736 & 1350 \\
\hline 45 & -116.99049 & 40.99599 & 1420 \\
\hline 60 & -116.95005 & 41.01850 & 1460 \\
\hline 43 & -116.88703 & 41.00584 & 1650 \\
\hline 42 & -116.80737 & 41.00304 & 1600 \\
\hline 59 & -116.64933 & 40.98375 & 1530 \\
\hline 58 & -116.54706 & 40.98970 & 1650 \\
\hline 57 & -116.45419 & 41.00731 & 1700 \\
\hline 34 & -116.41038 & 40.98637 & 1600 \\
\hline 37 & -116.39134 & 40.98808 & 1650 \\
\hline 33 & -116.36519 & 40.98794 & 1700 \\
\hline 32 & -116.33897 & 40.99409 & 1800 \\
\hline 35 & -116.26639 & 40.99996 & 1800 \\
\hline 56 & -116.22838 & 40.99611 & 1740 \\
\hline
\end{tabular}

The figures in the Appendix represent the field-processed MT data for each station after the time series data were converted to the frequency domain and the tensor-transfer function was rotated into principal directions as described above in the "Magnetotelluric Method" section.

For each station, nine separate plots are given:

1. Apparent Resistivity for the rotated maximum ( $x$ symbol) and minimum (o symbol) modes

2. Impedance Phase for the rotated maximum ( $x$ symbol) and minimum (o symbol) modes

3. Rotation Angle for the impedance tensor (corresponds to the direction of maximum apparent resistivity)

4. Impedance skew for the impedance tensor

5. Multiple Coherency for the rotated maximum ( $x$ symbol) and minimum (o symbol) modes of the electric field

6. Impedance Polar Plots (at 12 selected frequencies)

7. Tipper Magnitude for the vertical magnetic field

8. Tipper strike for the vertical magnetic field, and

9. $\mathrm{HzHx}$ ( $x$ symbol) and HzHy (o symbol) Coherency

Error bars on the Apparent Resistivity, Impedance Phase, Skew, Tipper Magnitude, and Tipper Strike plots represent probable errors within one standard deviation of the sample variance (Gamble and others, 1979b).

Apparent resistivity is a measure of the magnitude of the electric field strength over the magnetic field strength for a 
given frequency. The impedance phase is proportional to the slope of the apparent resistivity curve on a log-log plot, but from a baseline at -45 degrees (Vozoff, 1991). A measure of the dimensionality for MT data is provided by the impedance skew of the impedance tensor (Vozoff, 1972). If the effective measured resistivity response to the geology beneath a MT station is truly $1-D$ or 2-D, then the skew will be zero. Both instrument and environmental sources of noise contribute to non-zero skew values, but are typically small (about 0.1) for relatively low noise level recordings. Higher skews (above 0.2) are an indication of either the resistivity response to 3-D geology or higher levels of noise. Man-made electrical noise, such as power lines, power generators, moving vehicles and trains can have a negative effect on MT data quality. All these local disturbances produce an incoherent noise mainly affecting frequencies above $1 \mathrm{~Hz}$. Other man-made electrical noise, such as direct current electric trains and active cathodic protection of pipelines produce coherent electromagnetic signals mainly affecting frequencies below 1 $\mathrm{Hz}$.

In the survey area, noise from a number of small power lines and small moving vehicles was negligible at distances of $0.4 \mathrm{~km}$ and greater from the noise source. Power line levels were measured at each site and were typically less than $20 \%$ of the maximum recordable signals. Noise from larger power lines, power generators, pipelines, railroads, and steamdriven trains, mostly near mining operations, was negligible at least $5 \mathrm{~km}$ from them. Recordings were not made when noise from moving vehicles affected the magnetic signals. Local lightning, wind, and rainstorms can also degrade data quality, but these were avoided by not recording during active thunderstorm periods. Wind noise was minimized by burying the magnetic induction coils.

Predicted values of the electric field can be computed from the measured values of the magnetic field (Vozoff, 1991). The coherence of the predicted electric field with the measured electric field is a measure of the signal-to-noise ratio provided in the multiple coherency plot. Values are normalized between 0 and 1 , where values at 0.5 signify signal levels equal to noise levels. For this data set, coherencies were generally at an acceptable level, except at times in the "dead band" (0.1 to $1 \mathrm{~Hz})$ and at times in the lower frequencies $(0.004$ to 0.1$)$. The lower frequency ionospheric signals are related to sunspot activity whose levels typically follow an 11-year cycle. The sunspot activity was near the highest level of the cycle during the 1998 and 2000 surveys. 
The figures in the Appendix represent the field-processed MT data at each station, which includes some data scatter and poor signal-to-noise ratios. Our only effort at removing noisy data points was to visually inspect and select the best signal-to-noise field data to combine into the final data plots.

The impedance polar plots provide a measure of the MT data dimensionality (Reddy and others, 1977). For 1-D resistivity structures, the principal impedance polar diagram (dashed line) is a circle. For 2-D or 3-D resistivity structures, the principal impedance polar diagram (dashed line) elongates either parallel or perpendicular to strike direction. Over resistors, the principal impedance polar diagram elongates perpendicular to strike direction and over conductors, the principal impedance polar diagram elongates parallel to strike direction. Also, for 2-D resistivity structures, the additional impedance polar diagram (solid line) attains the shape of a symmetric clover leaf. For 3-D resistivity structures, the additional impedance polar diagram (solid line) elongates in one direction and its amplitude is comparable to that of the principal impedance polar diagram (dashed line). Sites whose polar plots indicated 3-D character in the lower frequencies were MT stations 53, 42, $58,57,37,33$, and 32 (Figure 1). MT station 61 was the only site whose polar plots indicated 3-D character in all the frequencies measured (from 100 to $.008 \mathrm{~Hz}$ ).

The tipper can be calculated when the vertical component of the magnetic field is measured. The tipper magnitude is a measure of the tipping of the magnetic field out of the horizontal plane (Vozoff, 1991). The magnitude is zero for the 1-D case and typically increases between 0.1 to 0.5 , and rarely as great as 1 , as it responds to vertical and subvertical structures. The tipper strike is typically used to help resolve the 90-degree ambiguity in the impedance rotation angle. The vertical component of the magnetic field was measured only at stations 53, 48, 59, and 37. The tipper magnitude of these stations was typically 0.1 to 0.4 over the lower frequencies indicating vertical structure at depth. Station 37 had even larger tipper magnitudes (over 0.5) in the higher frquencies indicating nearby vertical structure at depth. The $\mathrm{HzHx}$ and $\mathrm{HzHy}$ coherency is a measure of the signal-to-noise ratio of the vertical magnetic field with respect to each of the orthogonal horizontal magnetic field directions. Values are normalized between 0 and 1 , where values at 0.5 signify signal levels equal to noise levels. 
These three-component magnetic field coherencies provide a check on the signal-to-noise ratio of the measured values in the tipper magnitude and tipper strike plots.

\section{REFERENCES CITED}

Arehart, G.B., Foland, K.A., Naeser, C.W., and Kesler, S.E., 1993, 40Ar/39Ar, K/Ar, and fission track geochronology of sediment-hosted disseminated gold deposits at Post-Betze, Carlin Trend, northeastern Nevada: Economic Geology, vol. 88, no. 3, p. 622-646.

Bendat, J.S., and Piersol, A.G., 1971, Random Data: Analysis and Measurement Procedures: New York, Wiley Interscience, $407 \mathrm{p}$.

Clarke, J., Gamble, T.D., Goubau, W.M., Koch, R.H., and Miracky, R.F., 1983, Remote-reference magnetotellurics: Equipment and procedures: Geophysical prospecting, vol. 31, p. 149-170.

Dobrin, M.D., and Savit, C.H., 1988, Introduction to Geophysical Prospecting (4th ed.): New York, McGraw-Hill, $867 \mathrm{p}$.

Eberhart-Phillips, D., Stanley, W. D., Rodriguez, B. D. and Lutter, W. J., 1995, Surface seismic and electrical methods to detect fluids related to faulting: Journal of Geophysical Research, vol. 100, no. B7, pp. 12,91912,936 .

EMI, 1996, MT-1 magnetotelluric system operation manual, version 3.2: ElectroMagnetic Instruments, Inc., Richmond, California, $220 \mathrm{p}$.

Gamble, T.D., Goubau, W.M. and Clarke, J., 1979a, Magnetotellurics with a remote magnetic reference: Geophysics, v. 44, no. 1, p. 53-68.

Gamble, T.D., Goubau, W.M. and Clarke, J., 1979b, Error analysis for remote reference magnetotellurics: Geophysics, v. 44, no. 5, p. 959-968.

Ilchik, R.P. and Barton, M.D., 1997, An amagmatic origin of Carlin-type gold deposits: Economic Geology, vol. 92, no. 3 , p. 269-288. 
Keller, G.V., 1987, Rock and mineral properties, in

Electromagnetic Methods in Applied Geophysics Theory: M.N. Nabighian, Ed., Society of Exploration Geophysicists, Tulsa, Oklahoma, v. 1, p. 1351.

Keller, G.V., 1989, Electrical properties, in Carmichael, R.S., Ed., Practical handbook of physical properties of rocks and minerals: CRC Press, Boca Raton, Florida, p. 359-427.

Nelson, P.H. and Anderson, L.A., 1992, Physical properties of ash flow tuff from Yucca Mountain, Nevada: Journal of Geophysical Research, vol. 97, no. B5, p. 6823-6841.

Palacky, G.J., 1987, Resistivity characteristics of geologic targets, in Electromagnetic Methods in Applied Geophysics Theory: M.N. Nabighian, Ed., Society of Exploration Geophysicists, Tulsa, Oklahoma, vol. 1, p. 53129.

Radtke, A.S., 1985, Geology of the Carlin gold deposit, Nevada: U.S. Geological Survey Professional Paper 1267, $124 \mathrm{p}$.

Reddy, I.K., Rankin, D., and Phillips, R.J., 1977, Threedimensional modeliing in magnetotelluric and magnetic variational sounding: Geophysics Journal of the Royal Astronomical society, vol. 51, p. 313-325.

Rodriguez, B.D., Stanley, W.D., and Williams, J.M., 1996, Axial structures within the Reelfoot rift delineated with magnetotelluric surveys: U.S. Geological Survey Professional Paper $1538-\mathrm{K}, 30 \mathrm{p}$.

Shawe, D.R., 1991, Structurally controlled gold trends imply large gold resources in Nevada, in Geology and ore deposits of the Great Basin, Symposium Proceedings: Raines, G.L., Lisle, R.E., Schafe, R.W., Wilkinson, W.H., Eds., Geological Society of Nevada, Reno, vol. 1, p. 199212 .

Sillitoe, R.H. and Bonham, H.F., 1990, Sediment-hosted gold deposits; distal products of magmatic-hydrothermal systems: Geology, vol. 18, no. 2, p. 157-161.

Stanley, W.D., 1978, New magnetotelluric field processing system: U.S. Geological Survey Professional Paper 1100, $174 \mathrm{p}$. 
Stanley, W.D., and Tinkler, R.D., 1983, A practical, low-noise coil system for magnetotellurics: U.S. Geological Survey Open-File Report 83-85, $18 \mathrm{p}$.

Sternberg, B.K., Washburne, J.C., and Pellerin, L., 1988, Correction for the static shift in magnetotellurics using transient electromagnetic soundings: Geophysics, vol. 53, p. $1459-1468$.

Struhsacker, E.M., Jones, E., and Green, S.M., 1996, Roadside geology and precious-metal mineralization along the I-80 corridor, Reno to Elko, Nevada, in Struhsacker, E.M. and Green, S.M., eds., Geology and ore deposits of the American Cordillera - Field Trip Guidebook Compendium: Geological Society of Nevada, Reno, Nevada, p. 3.

Tosdal, R.M., 1998, Contributions to the gold metallogeny of northern Nevada: U.S. Geological Survey Open-File Report $98-338,290 \mathrm{p}$.

Vozoff, K., 1972, The magnetotelluric method in the exploration of sedimentary basins: Geophysics, vol. 37, p. 98-141.

Vozoff, K., 1991, The magnetotelluric method, in Electromagnetic methods in applied geophysics: M.N. Nabighian, Ed., Society of Exploration Geophysicists, Tulsa, Oklahoma, vol. 2, part B, p. 641-711. 


\section{APPENDIX \\ MAGNETOTELLURIC DATA PLOTS}

There are nine separate plots for stations 53, 48, 59, and 37 :

1. Apparent Resistivity for the rotated maximum ( $x$ symbol) and minimum (o symbol) modes

2. Impedance Phase for the rotated maximum ( $x$ symbol) and minimum (o symbol) modes

3. Rotation Angle for the impedance tensor (corresponds to the direction of maximum apparent resistivity)

4. Impedance skew for the impedance tensor

5. Multiple Coherency for the rotated maximum (x symbol) and minimum (o symbol) modes of the electric field

6. Impedance polar plots (at 12 selected frequencies)

7. Tipper Magnitude for the vertical magnetic field

8. Tipper Strike for the vertical magnetic field, and

9. $\mathrm{HzHx}$ (x symbol) and HzHy (o symbol) Coherency

All other stations have only the first six plots above, since the vertical magnetic field data (Tipper, Hz) was not acquired. Refer to the "Magnetotelluric Data" section in this report for an explanation of these plots. 


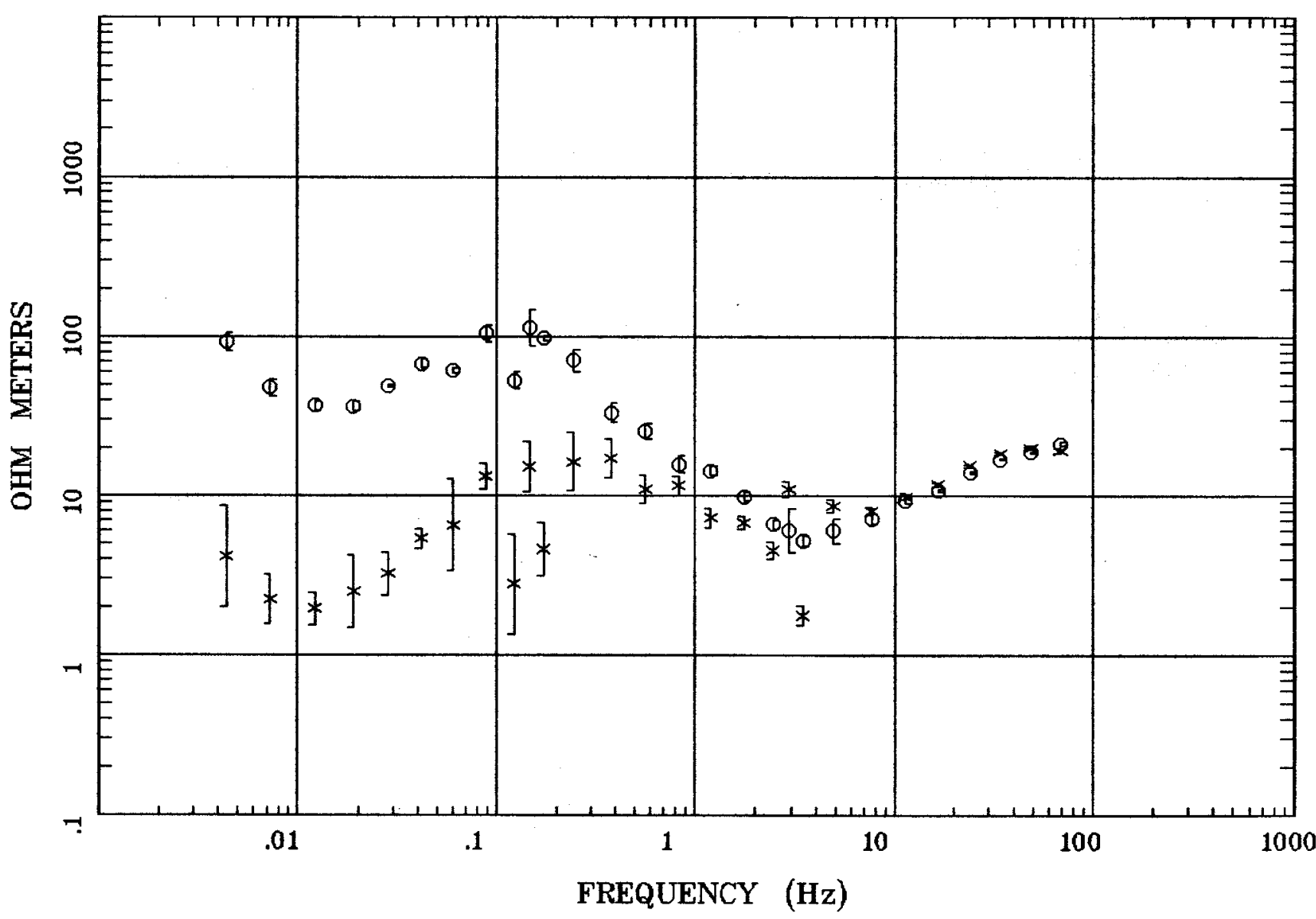

Client:

Remote: Local B

Acquired: 10:1 Jul 30, 1998 Survey Co:USGS GD-MRP Denver
Rotation:

Filename: hr53all.avg

Channels: Ch1 Ch2 Ch3 Ch4 Ch5 Ch8 Ch9 Plotted: 13:55 Dec 07, 2000

< EMI - ElectroMagnetic Instruments 


\section{Station 53}

IMPEDANCE PHASE

Humboldt River Line 1

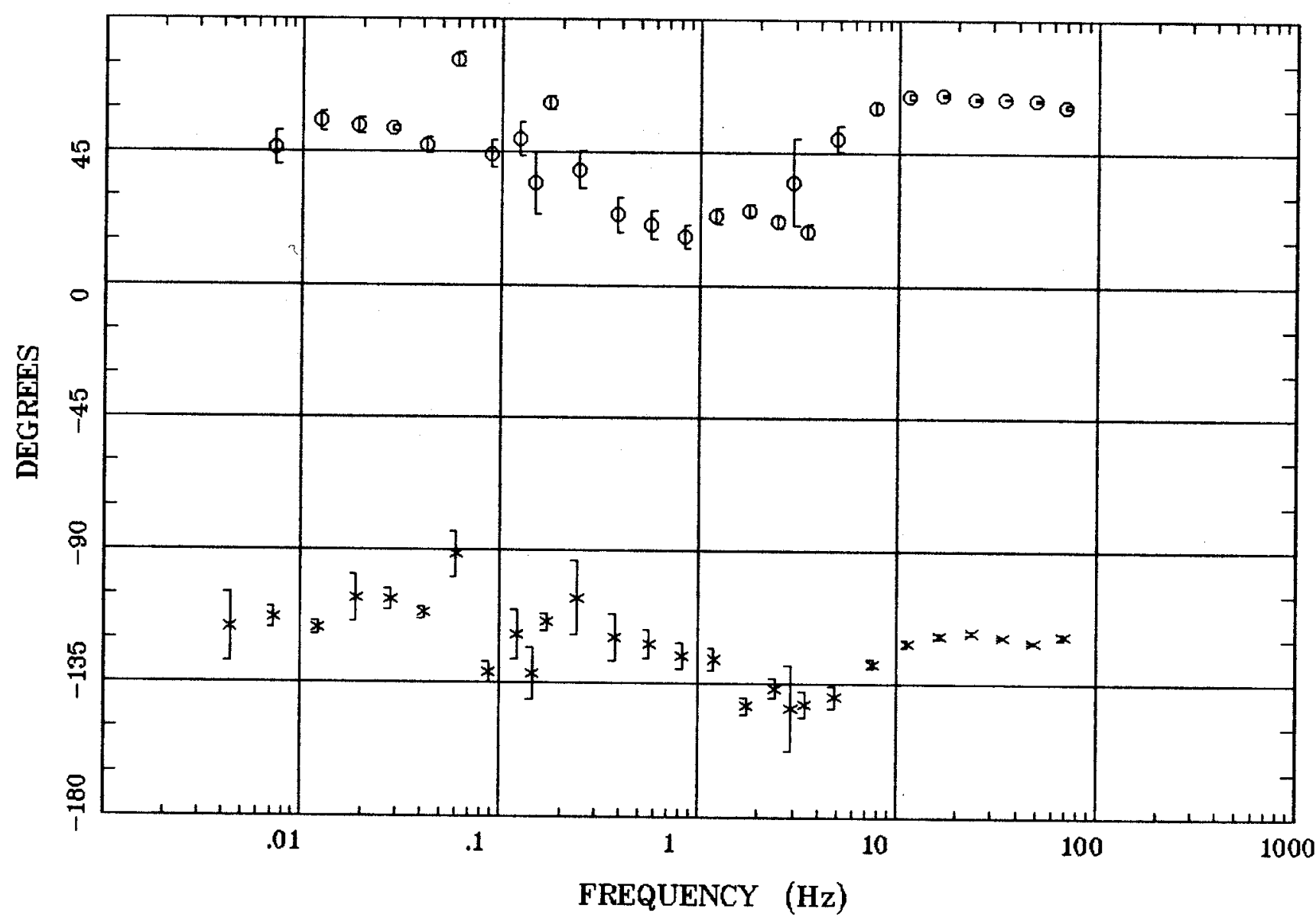

Client:

Remote: Local B

Acquired: $10: 1$ Jul 30,1998

Survey Co:USGS GD-MRP Denver
Rotation:

Filename: hr53all.avg

Channels: Ch1 Ch2 Ch3 Ch4 Ch5 Ch8 Ch9

Plotted: 13:55 Dec 07, 2000

< EMI - ElectroMagnetic Instruments > 


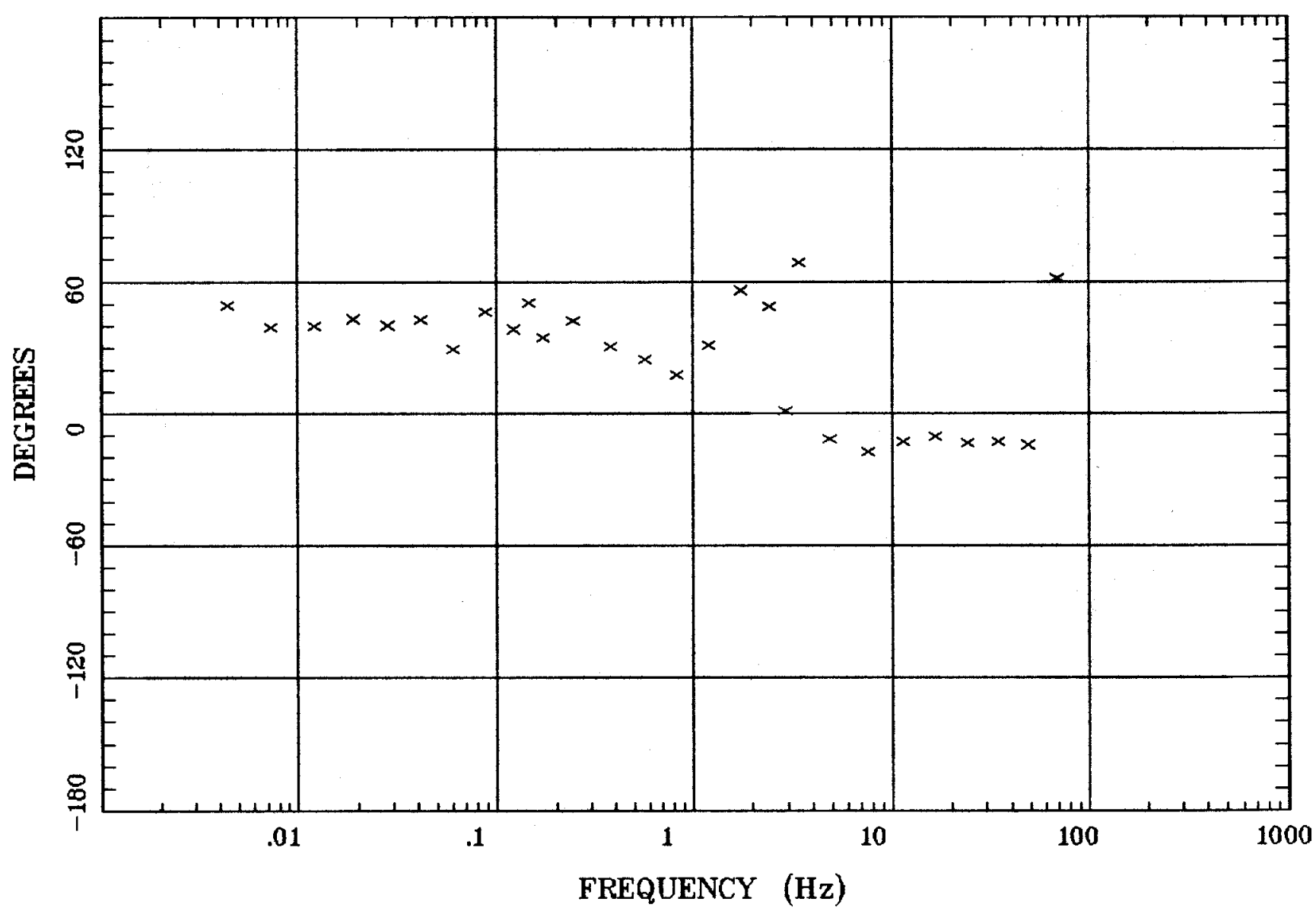

Client:

Remote: Local B

Acquired: 10:1 Jul 30, 1998 Survey Co:USGS GD-MRP Denver
Rotation:

Filename: hr53all.avg

Channels: Ch1 Ch2 Ch3 Ch4 Ch5 Ch8 Ch9

Plotted: 13:55 Dec 07, 2000

$<$ EMI - ElectroMagnetic Instruments 


\section{Station 53}

IMPEDANCE SKEW

Humboldt River Line 1

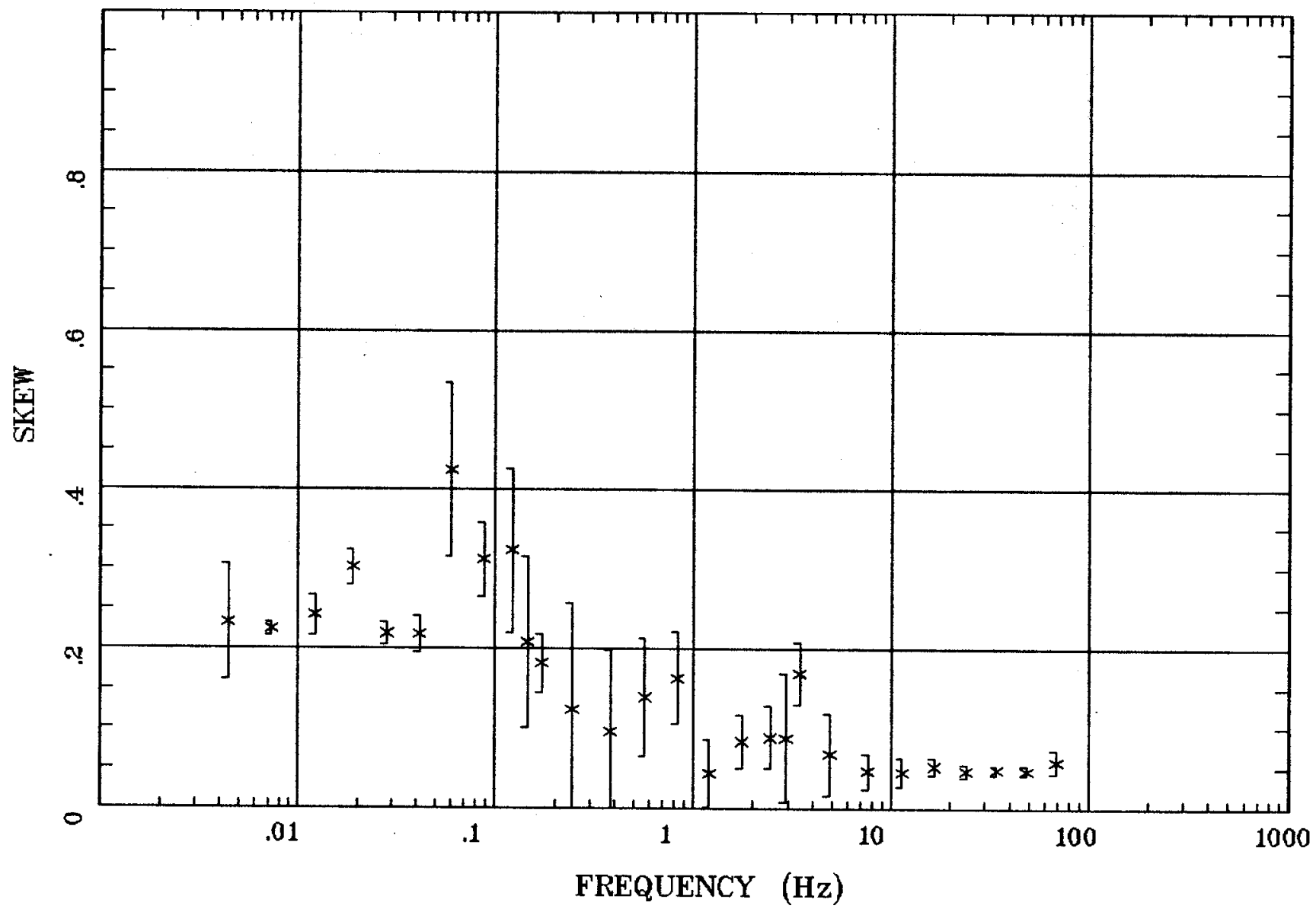

Client:

Remote: Local B

Acquired: 10:1 Jul 30, 1998

Survey Co:USGS GD-MRP Denver
Rotation:

Filename: hr53all.avg

Channels: Ch1 Ch2 Ch3 Ch4 Ch5 Ch8 Ch9

Plotted: 13:55 Dec 07, 2000

< EMI - ElectroMagnetic Instruments 


\section{Station 53}

E MULT Coh.

Humboldt River Line 1

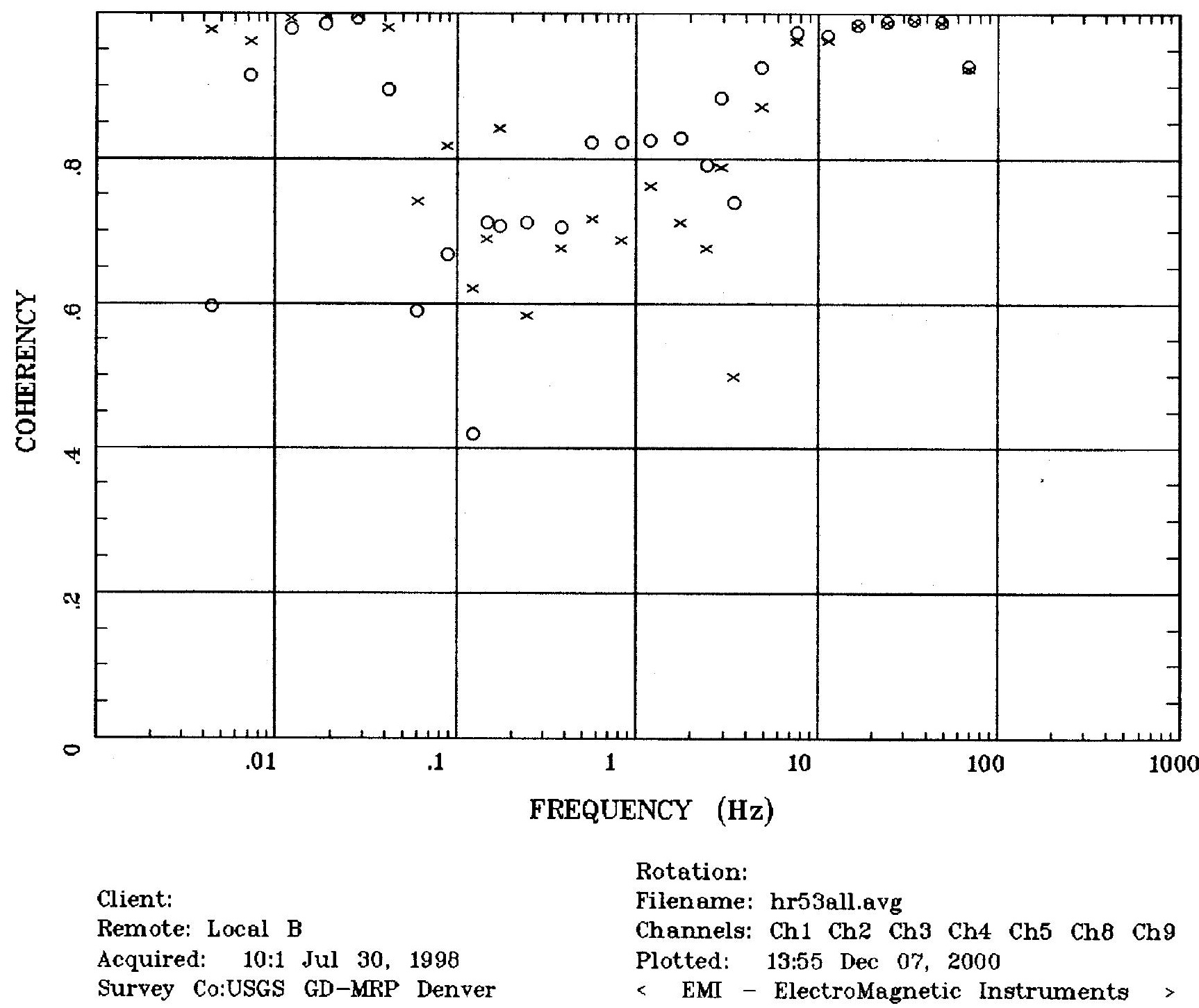




\section{POLAR PLOTS}

Humboldt River Line 1

Station 53

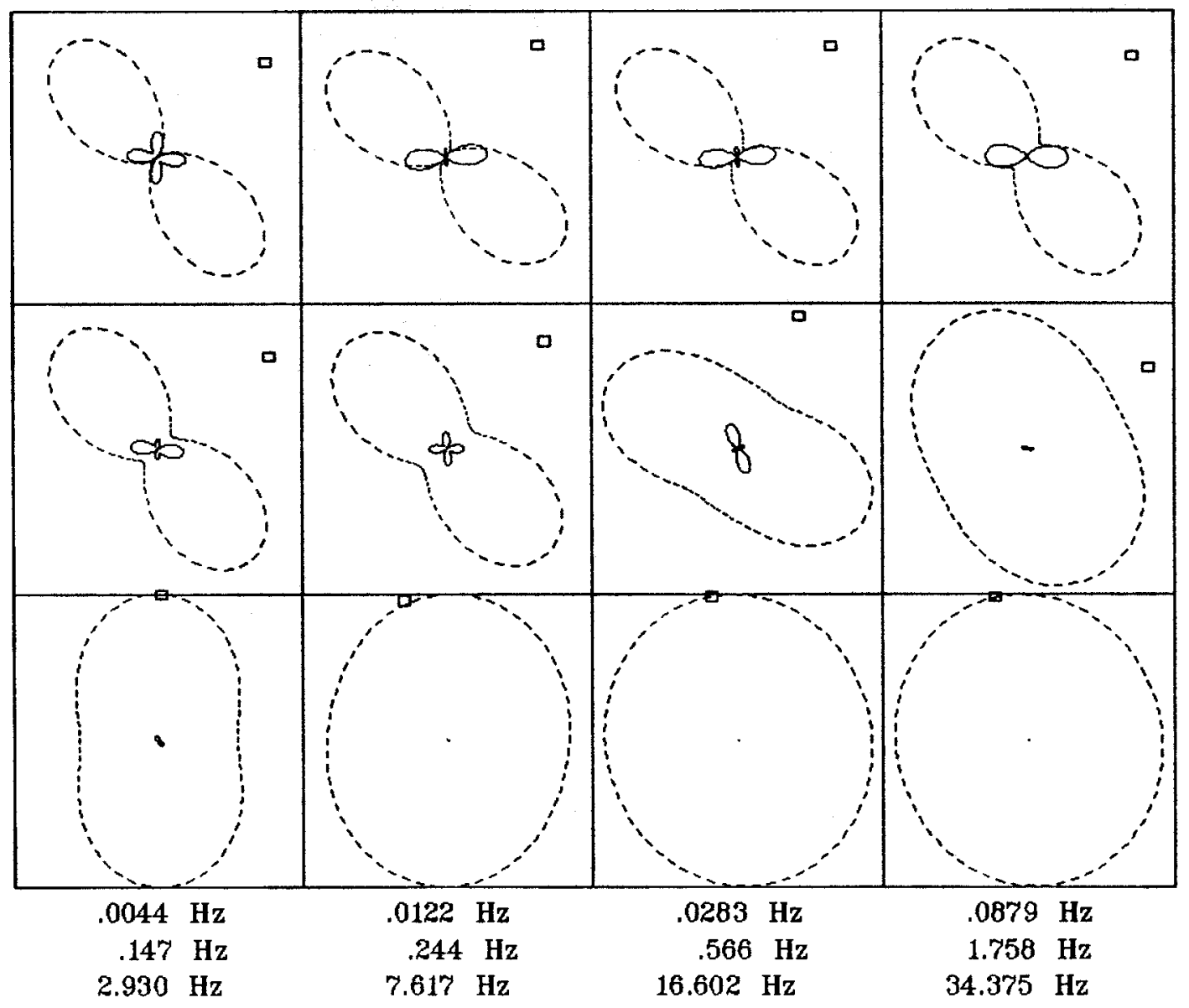

Rotation:

Client:

Remate: Local $B$

Acquired: 10:1 Jul 30, 1998

Survey Co:USGS GD-MRP Denver
Filename: hr53allavg

Channels: Ch1 Ch2 Ch3 Ch4 Ch5 Ch8 Ch9

Plotted: 13:55 Dec 07, 2000

< EMI - ElectroMagnetic Instruments > 


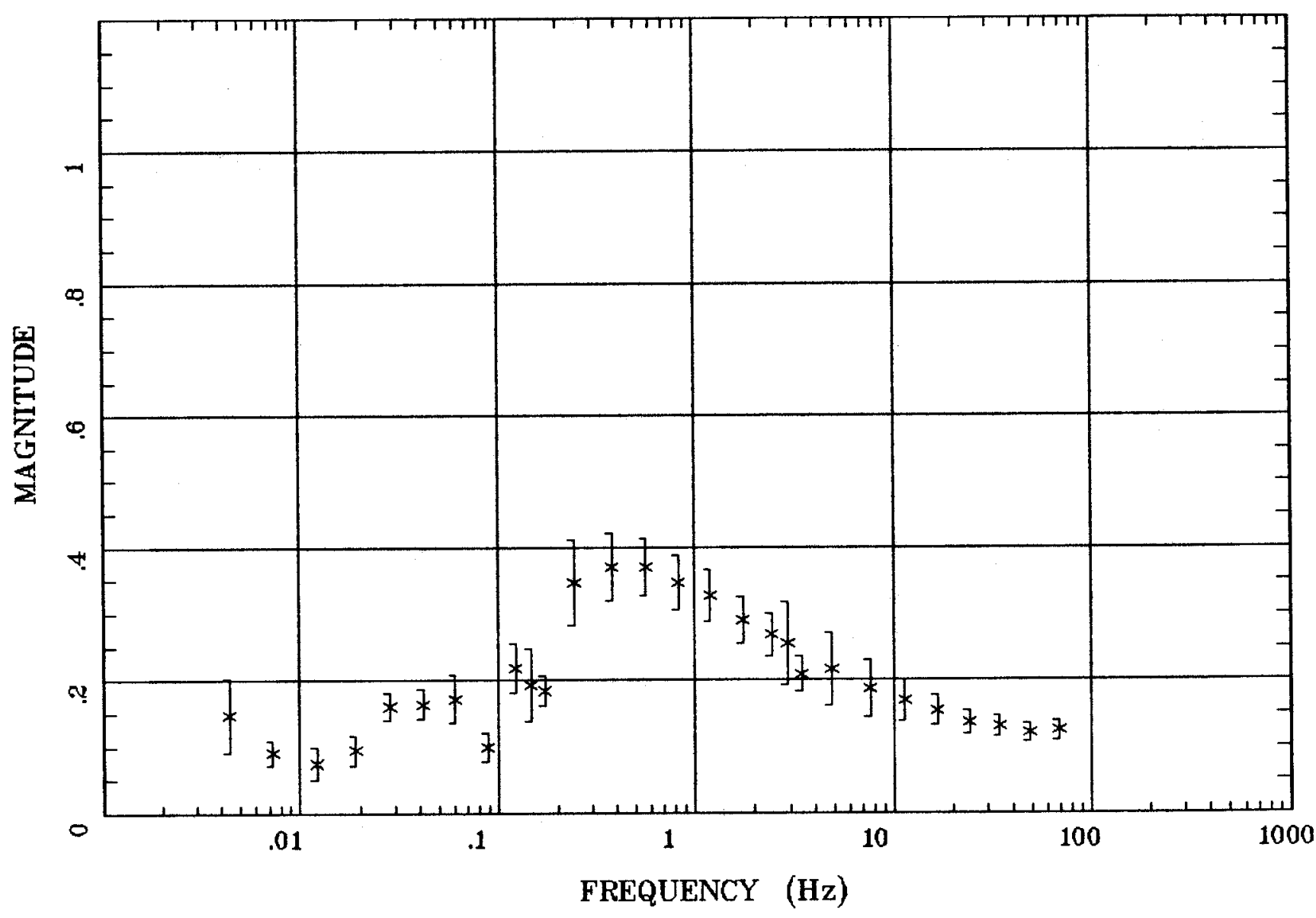

Client:

Remote: Local B

Acquired: $10: 1 \mathrm{Jul} 30,1998$ Survey Co:USGS GD-MRP Denver
Rotation:

Filename: hr53all.avg

Channels: Ch1 Ch2 Ch3 ch4 Ch5 Ch8 Ch9 Plotted: 13:55 Dec 07, 2000

< EMI - ElectroMagnetic Instruments 


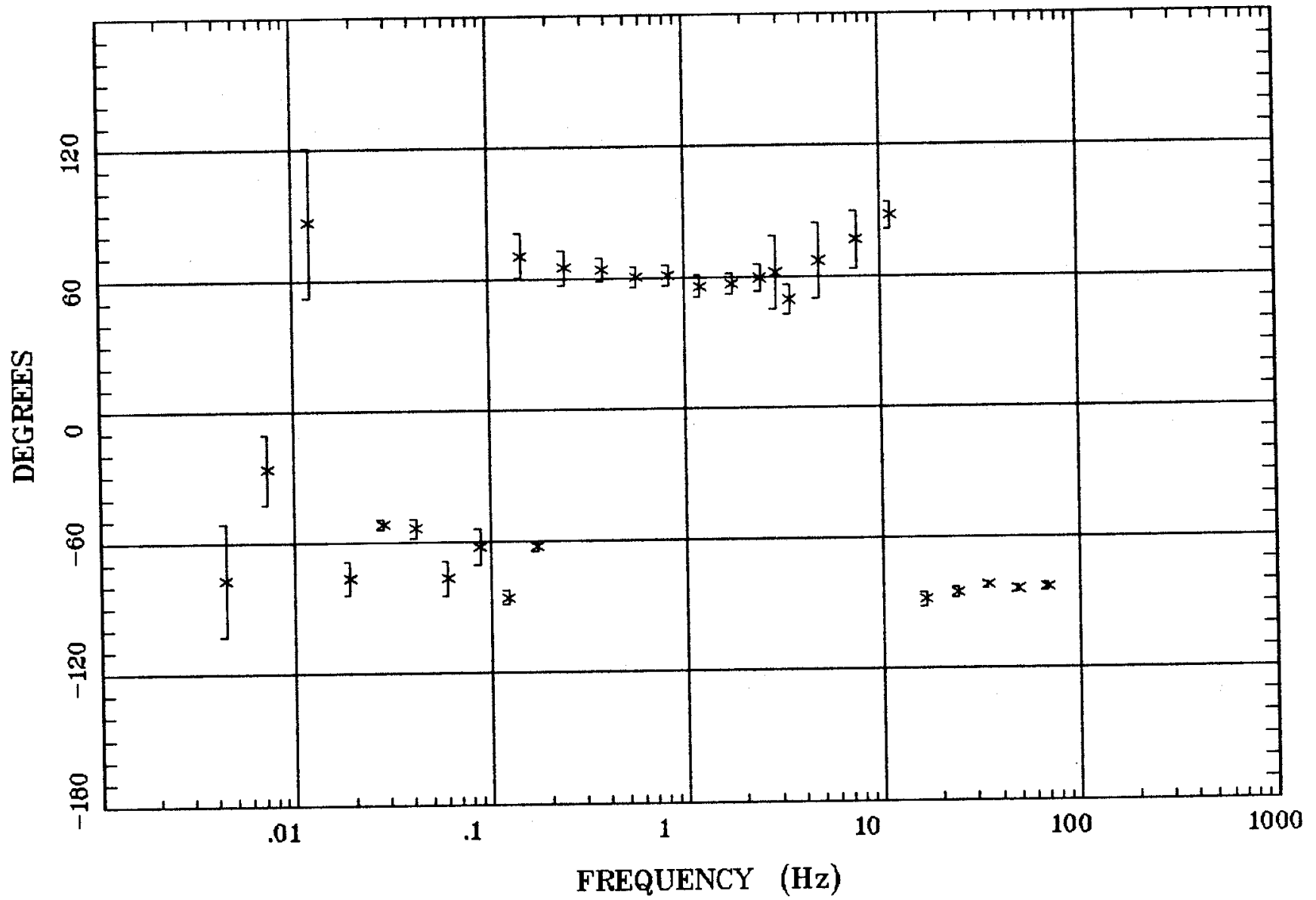

Client:

Remote: Local B Acquired: 10:1 Jul 30, 1998 Survey Co:USGS GD-MRP Denver
Humboldt River Line 1

\section{Rotation:}

Filename: hr53all.avg

Channels: Ch1 Ch2 Ch3 Ch4 Ch5 Ch8 Ch9

Plotted: 13:55 Dec 07, 2000

< EMI - ElectroMagnetic Instruments > 


\section{HzHx.x Coh HzHy.o}

\section{Humboldt River Line 1}

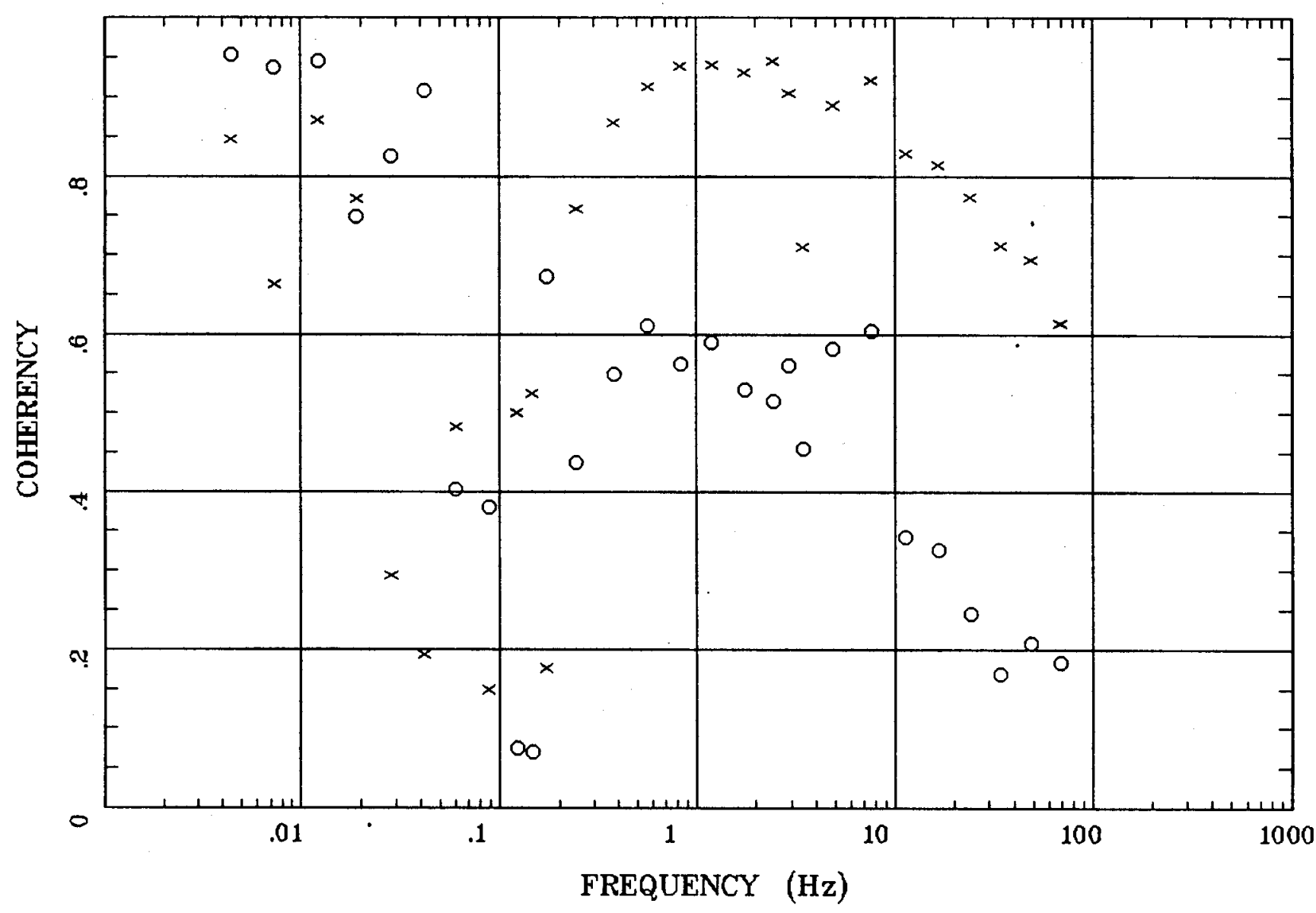

Client:

Remote: Local B

Acquired: 10:1 Jul 30, 1998 Survey Co:USGS GD-MRP Denver
Rotation:

Filename: hr53all.avg

Channels: Ch1 Ch2 Ch3 Ch4 Ch5 Ch8 Ch9 Plotted: 15:54 Dec 07, 2000

< EMI - ElectroMagnetic Instruments > 


\section{Station 61}

\section{APPARENT RESISTIVITY}

Humboldt River Line 1

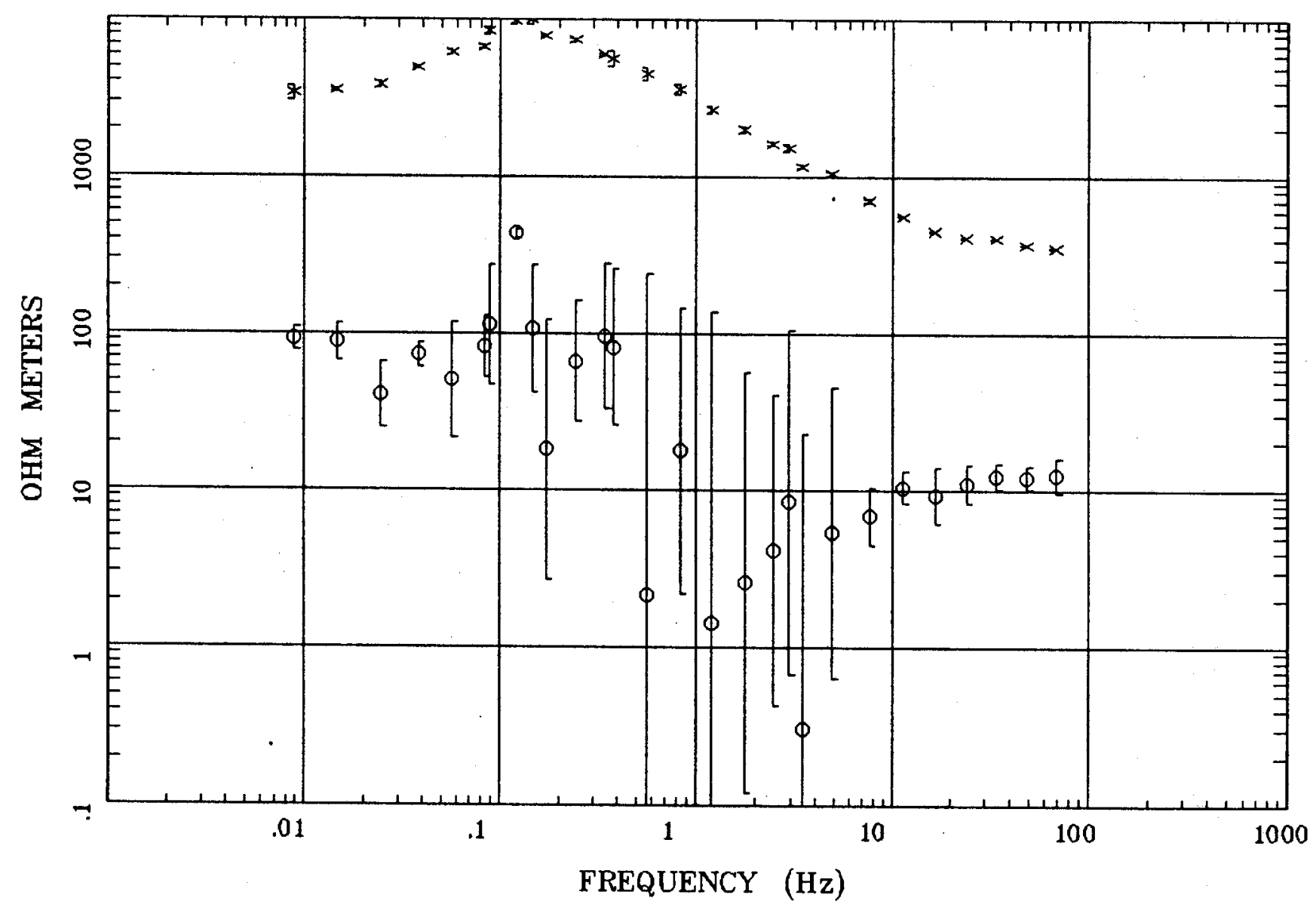

Client:

Remote: Local B

Acquired: 09:5 Aug 07, 1998

Survey Co:USGS GD-MRP Denver
Rotation:

Filename: hr61.avg

Channels: Ch1 Ch2 Ch3 Ch4 Ch5 Ch8 Ch9

Plotted: 14:04 Dec 07, 2000

$<$ EMI - ElectroMagnetic Instruments 


\section{Station 61}

IMPEDANCE PHASE

Humboldt River Line 1

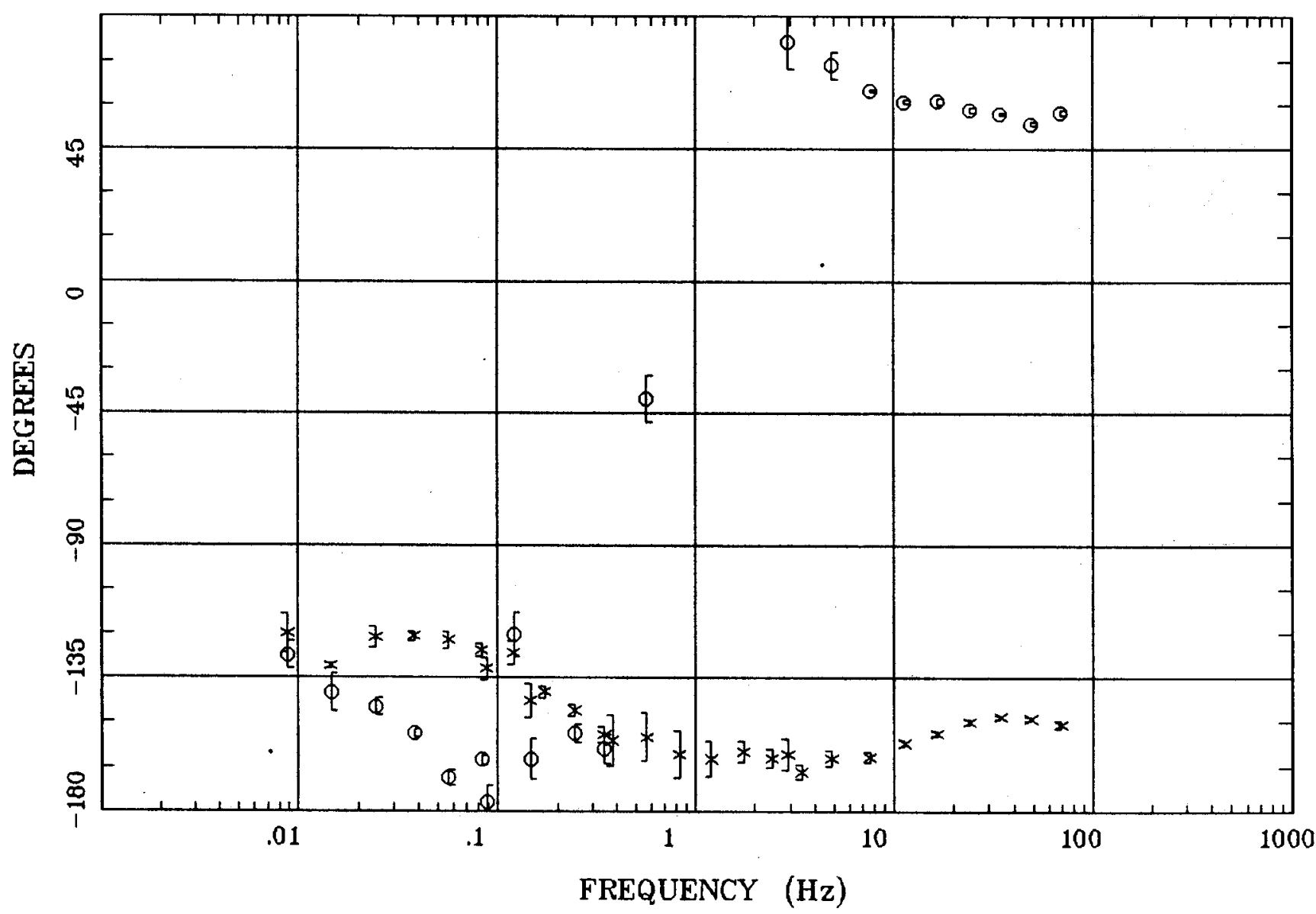

Client:

Remote: Local B

Acquired: 09:5 Aug 07, 1998

Survey Co:USGS GD-MRP Denver
Rotation:

Filename: hr61.avg

Channels: Ch1 Ch2 Ch3 Ch4 Ch5 Ch8 Ch9

Plotted: 14:04 Dec 07, 2000

$<$ EMI - ElectroMagnetic Instruments > 


\section{Station 61}

ROTATION ANGLE

Humboldt River Line 1

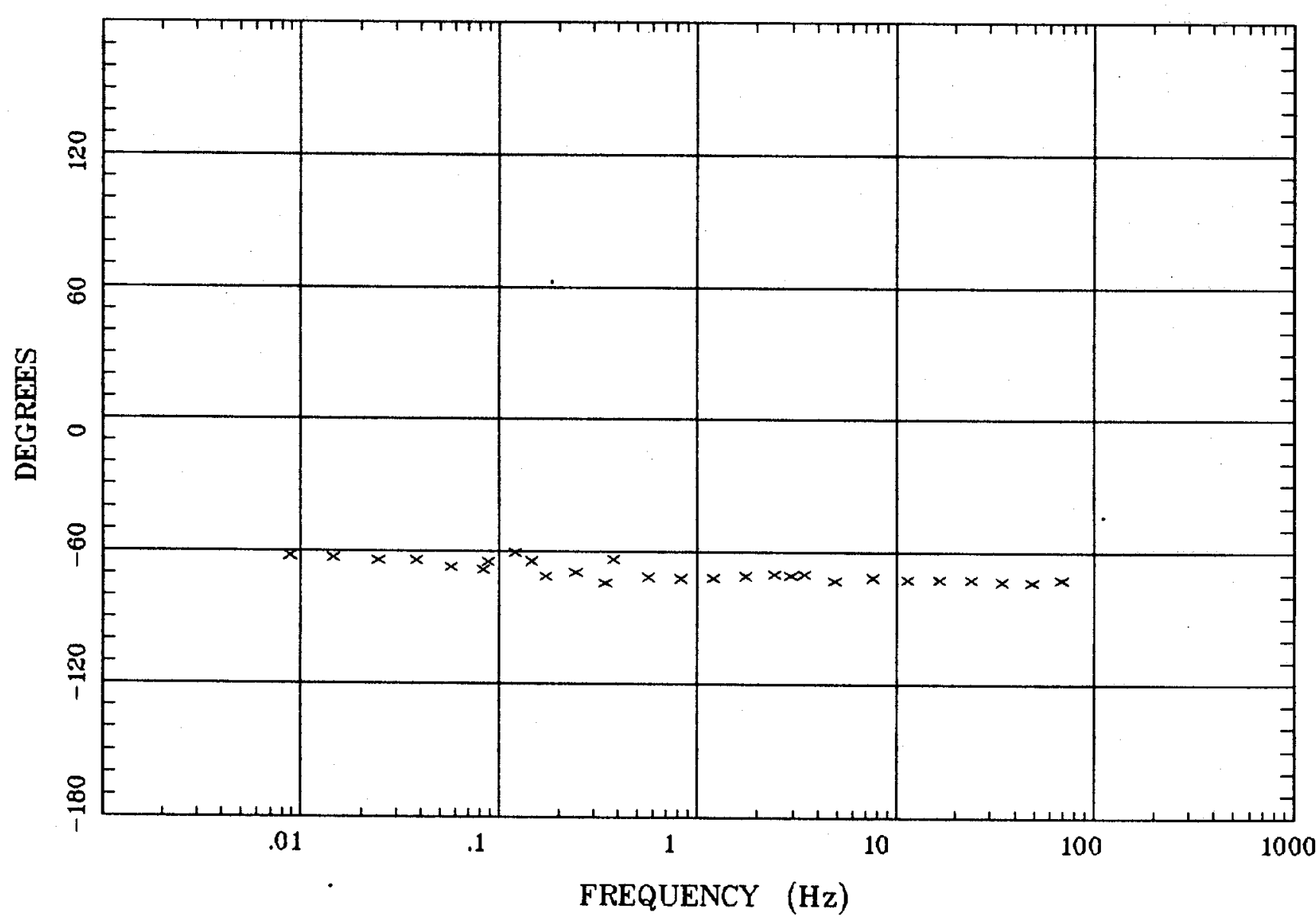

Client:

Remote: Local B

Acquired: 09:5 Aug 07, 1998

Survey Co:USGS GD-MRP Denver
Rotation:

Filename: hr61.avg

Channels: Ch1 Ch2 Ch3 Ch4 Ch5 Ch8 Ch9

Plotted: 14:04 Dec 07, 2000

$<$ EMI - ElectroMagnetic Instruments 
Station 61

IMPEDANCE SKEW

Humboldt River Line 1

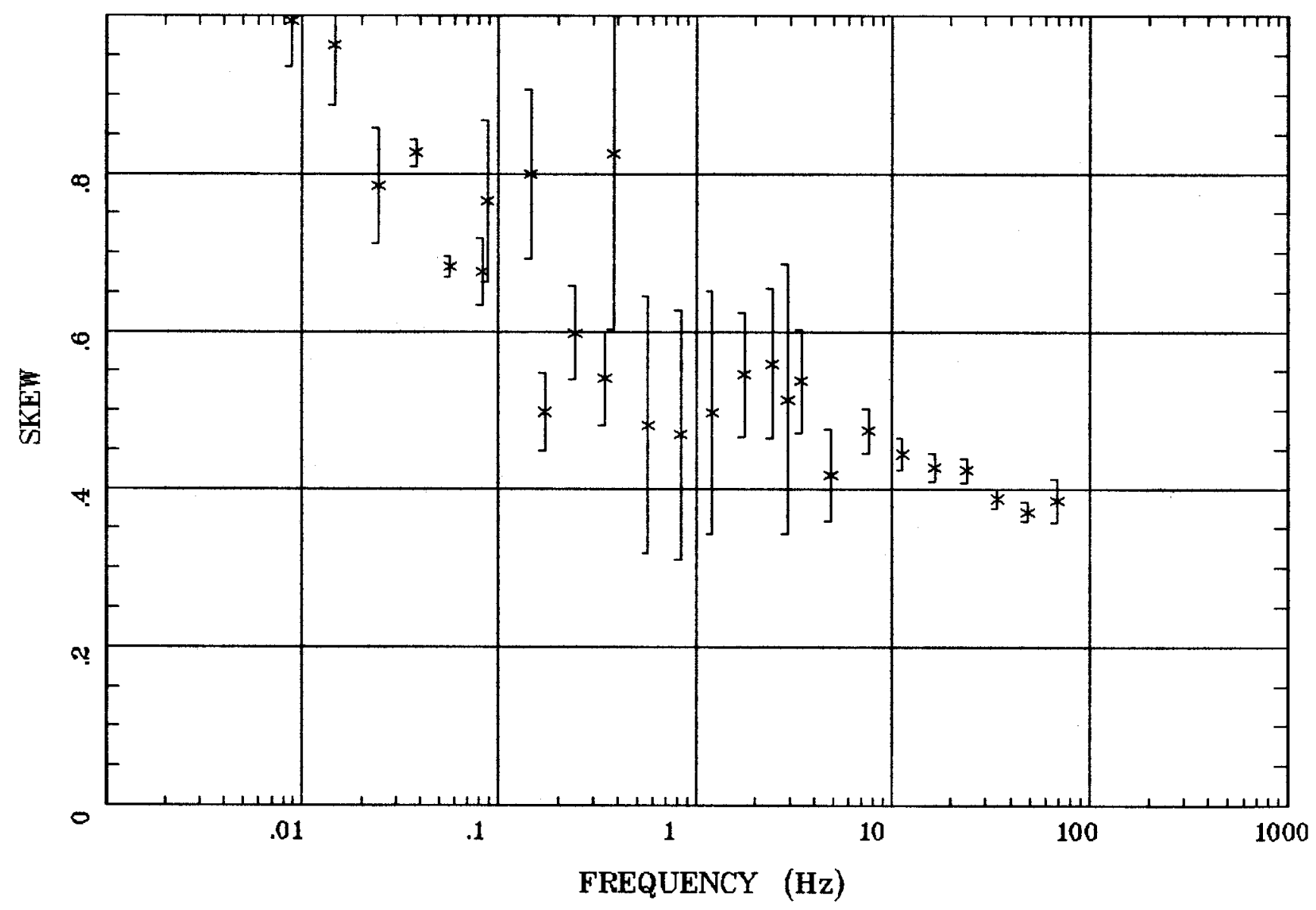

Client:

Remote: Local B

Acquired: 09:5 Aug 07, 1998 Survey Co:USGS GD-MRP Denver

Rotation:

Filename: hr61.avg

Channels: Ch1 Ch2 Ch3 Ch4 Ch5 Ch8 Ch9

Plotted: 14:04 Dec 07, 2000

< EMI - ElectroMagnetic Instruments 
Station 61

E MULT Coh.

Humboldt River Line 1

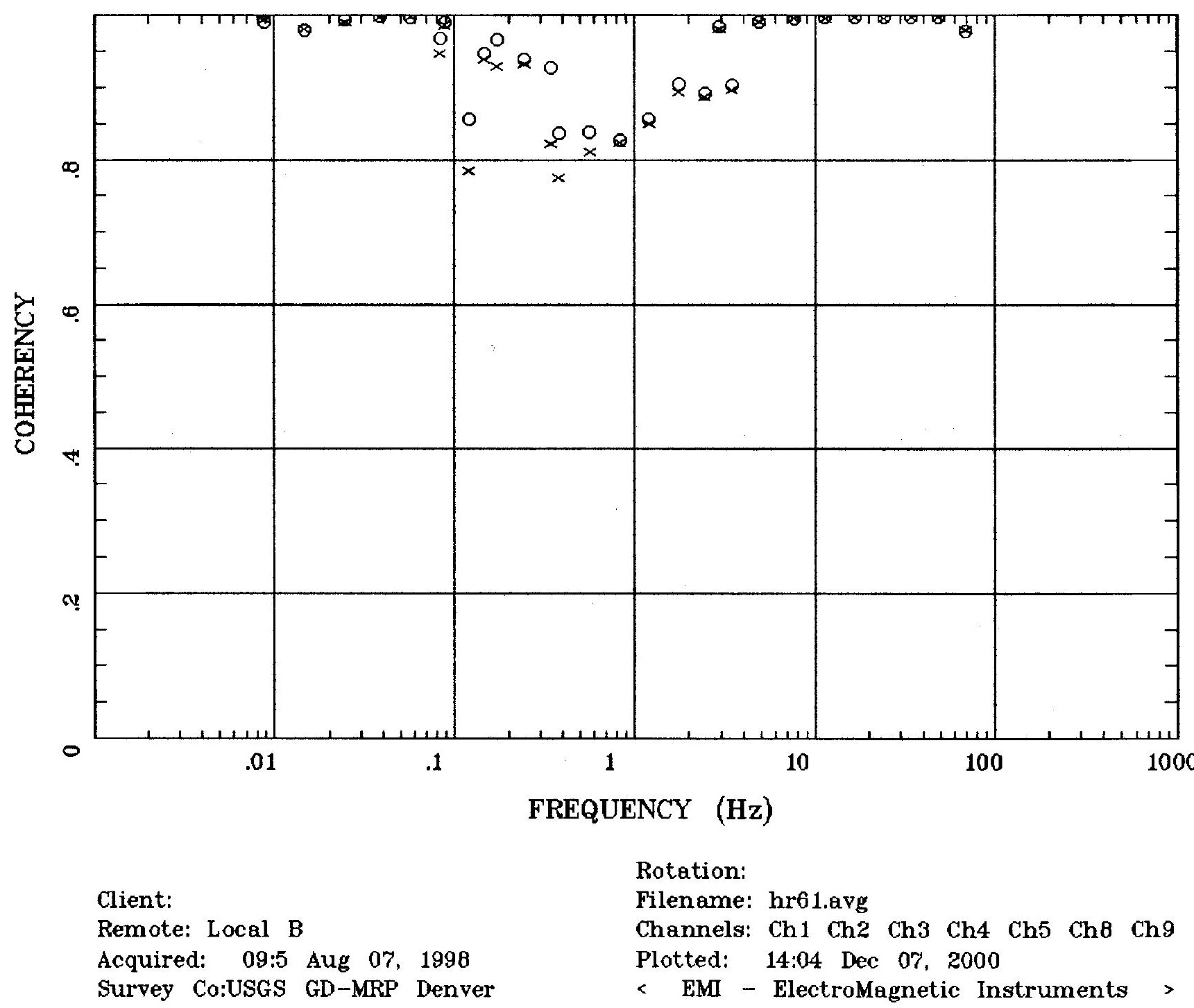




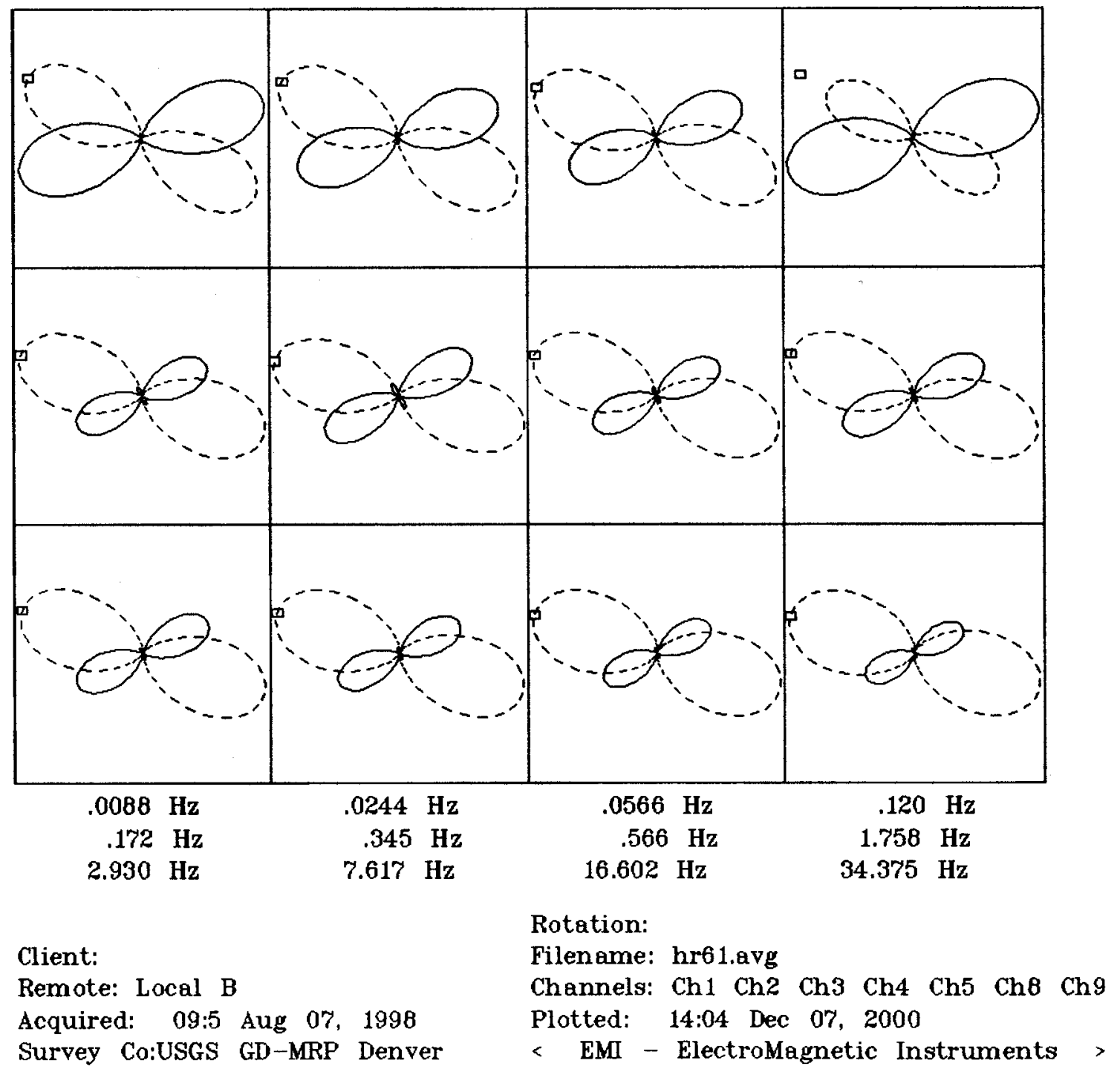




\section{APPARENT RESISTIVITY Osgood Mtns, NV 100K}

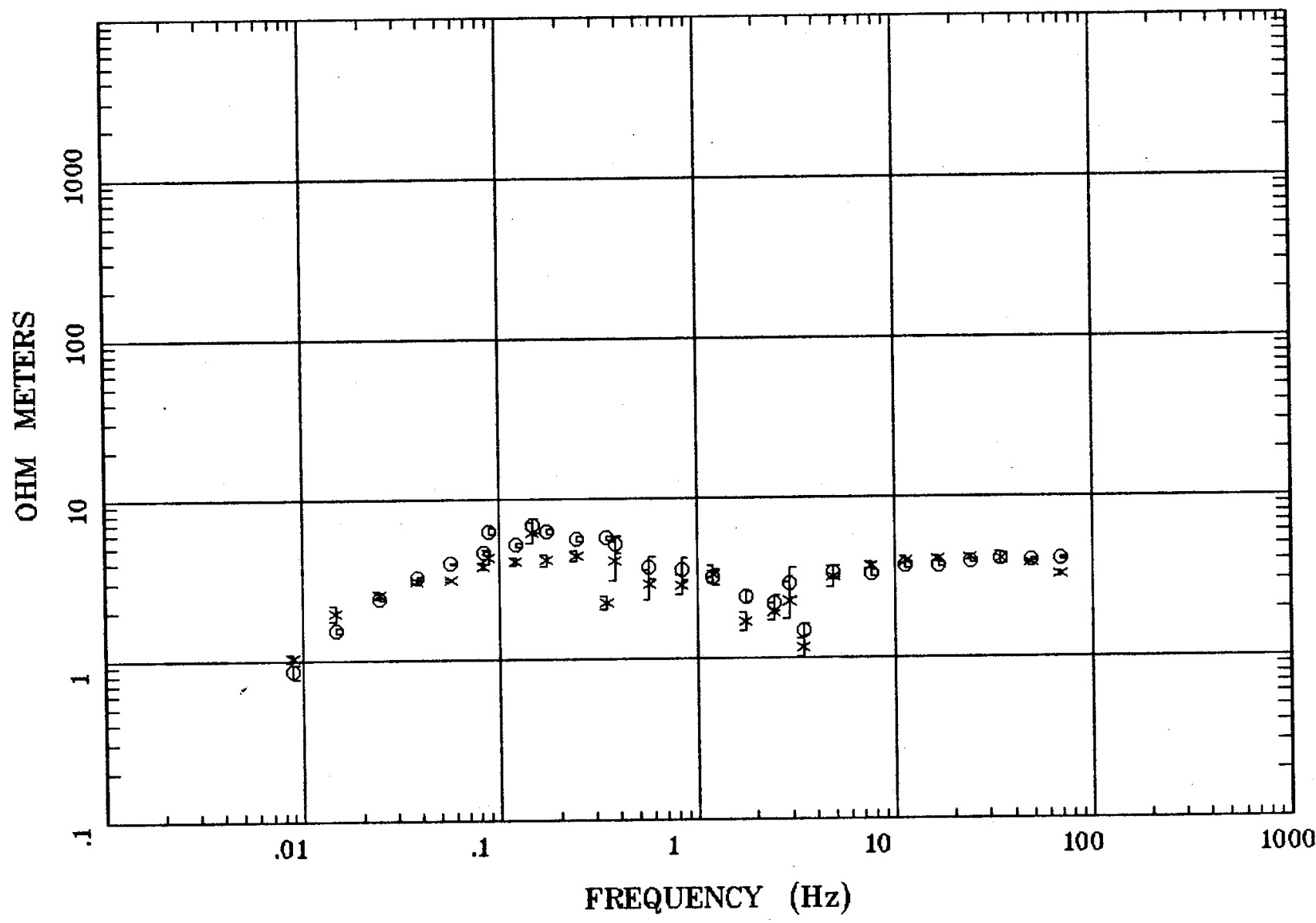

Client:

Remote: none

Acquired: $10: 4$ Jul 22, 2000 Survey Co:USGS
Rotation:

Filename: hr48c.avg

Channels: Ch1 Ch2 Ch3 Ch4 Ch5 Ch3 Ch4 Plotted: 11:05 Feb 06, 2001

$<$ EMI - ElectroMagnetic Instruments > 
IMPEDANCE PHASE

Osgood Mtns, NV 100K

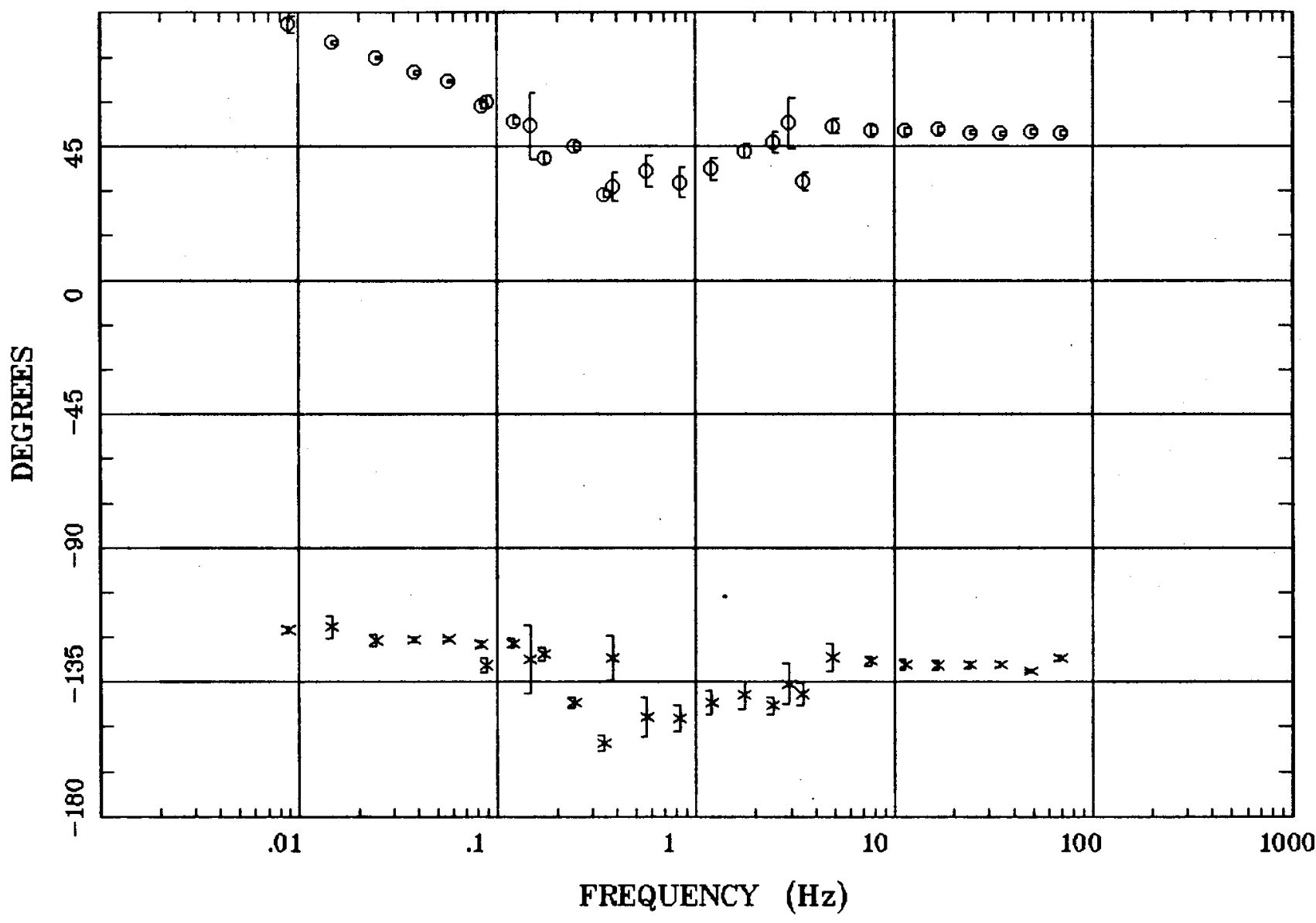

Client:

Remote: none

Acquired: 10:4 Jul 22, 2000

Survey Co:USGS
Rotation:

Filename: hr48c.avg

Channels: Ch1 Ch2 Ch3 Ch4 Ch5 Ch3 Ch4

Plotted: 11:05 Feb 06, 2001

< EMI - ElectroMagnetic Instruments 


\section{Station 48}

ROTATION ANGLE

Osgood Mtns, NV 100K

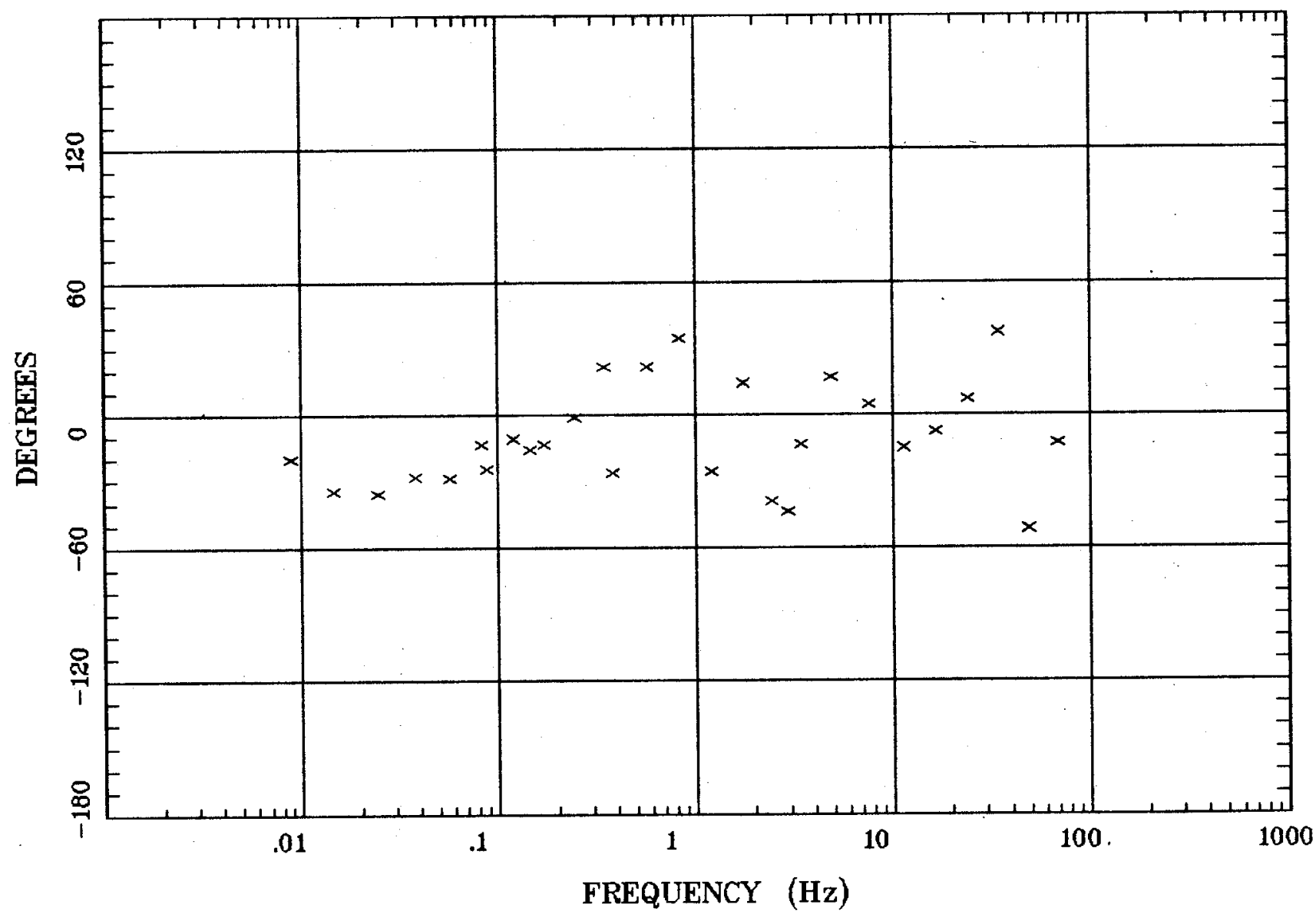

Client:

Remote: none

Acquired: 10:4 Jul 22, 2000 Survey Co:USGS
Rotation:

Filename: hr48c.avg

Channels: Ch1 Ch2 Ch3 Ch4 Ch5 Ch3 Ch4 Plotted: 11:05 Feb 06, 2001

< EMI - ElectroMagnetic Instruments > 


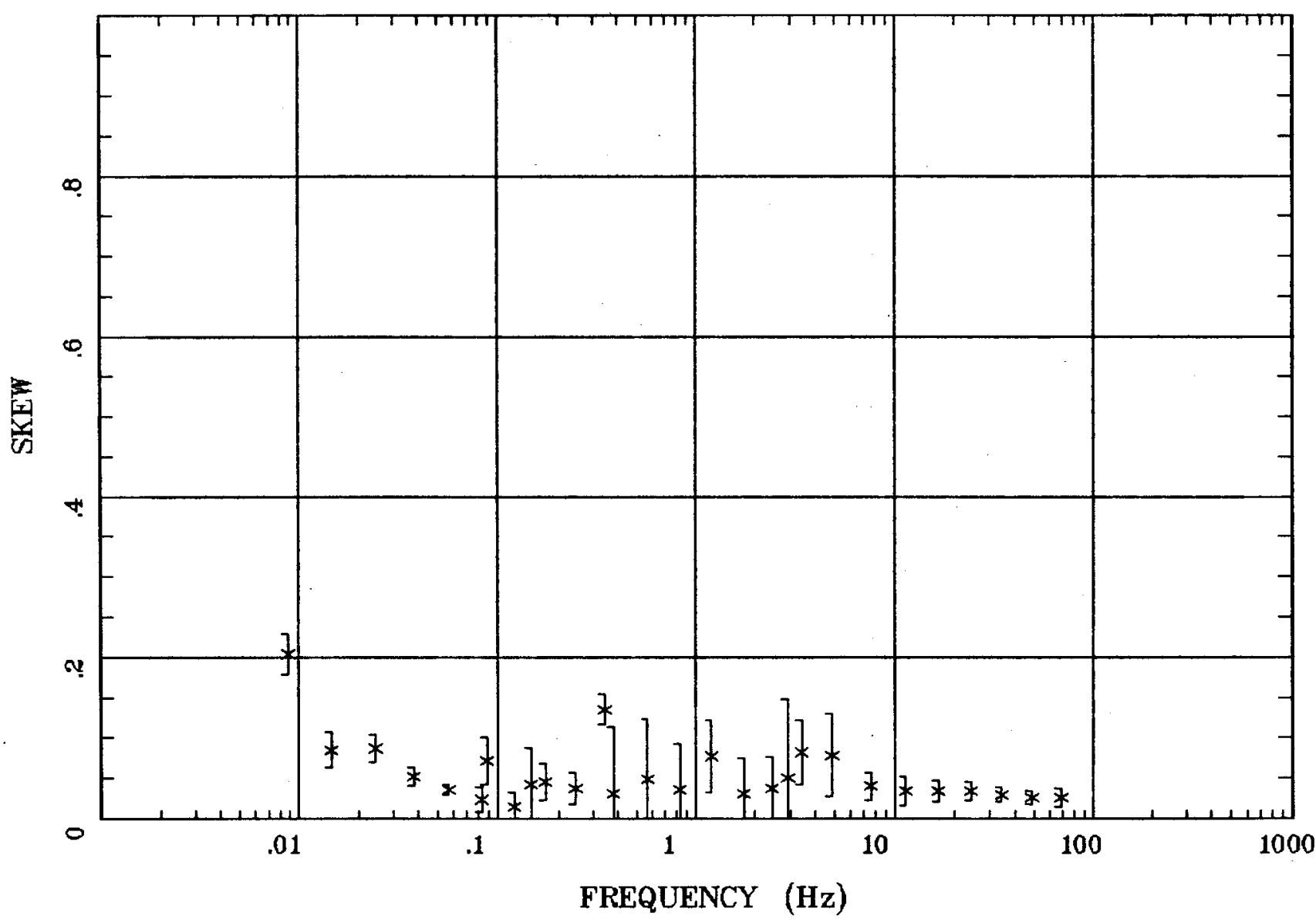

Client:

Remote: none

Acquired: 10:4 Jul 22, 2000 Survey Co:USGS
Rotation:

Filename: hr48c.avg

Channels: Ch1 Ch2 Ch3 Ch4 Ch5 Ch3 Ch4

Plotted: 11:05 Feb 06, 2001

$<$ EMI - ElectroMagnetic Instruments 
E MULT Coh. Osgood Mtns, NV 100K

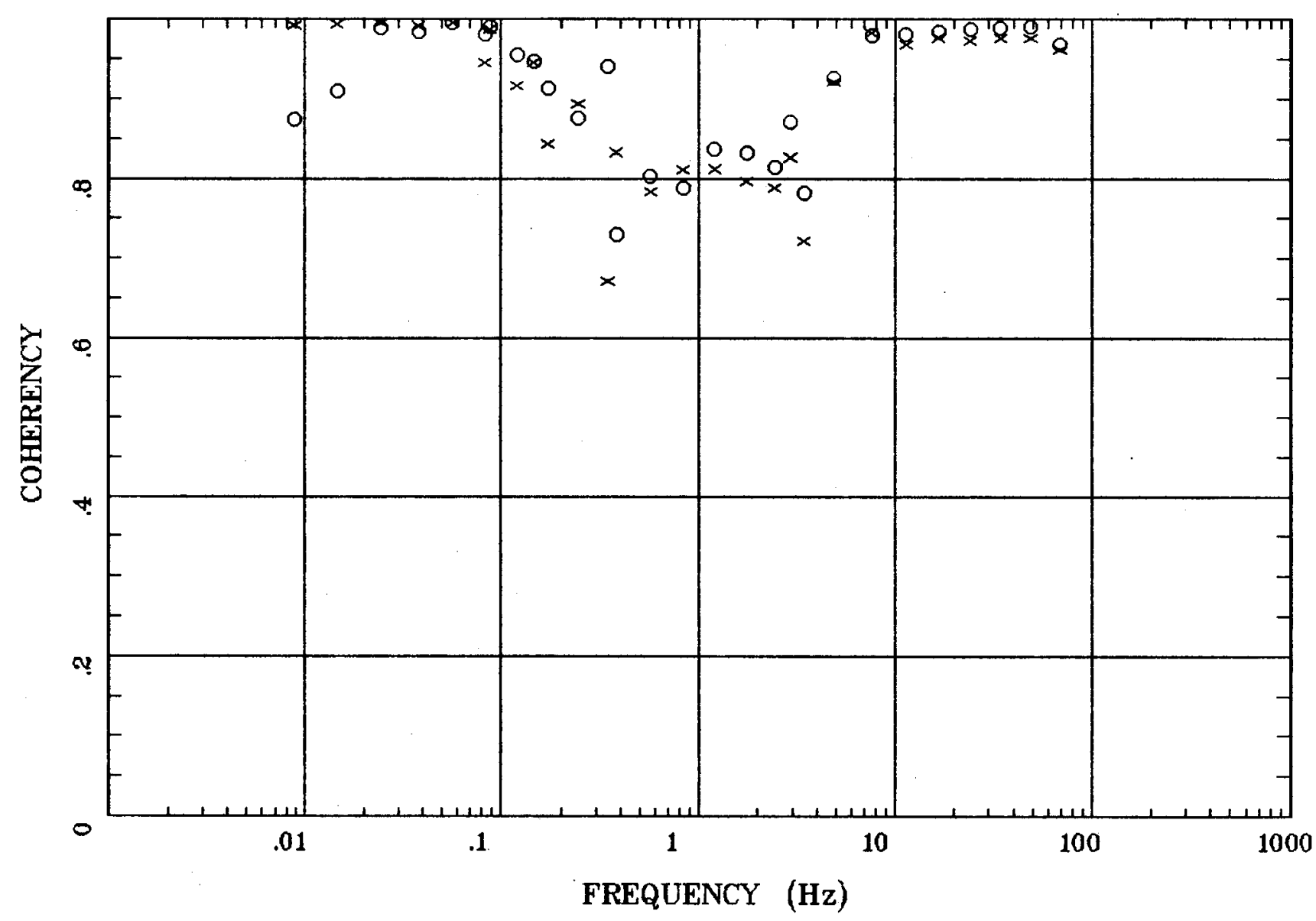

Client:

Remote: none

Acquired: 10:4 Jul 22, 2000 Survey co:USGS
Rotation:

Filename: hr48c.avg

Channels: Ch1 Ch2 Ch3 Ch4 Ch5 Ch3 Ch4

Plotted: 11:05 Feb 06, 2001

$<$ EMI - ElectroMagnetic Instruments 


\section{POLAR PLOTS}

Osgood Mtns, NV 100K

Station 48

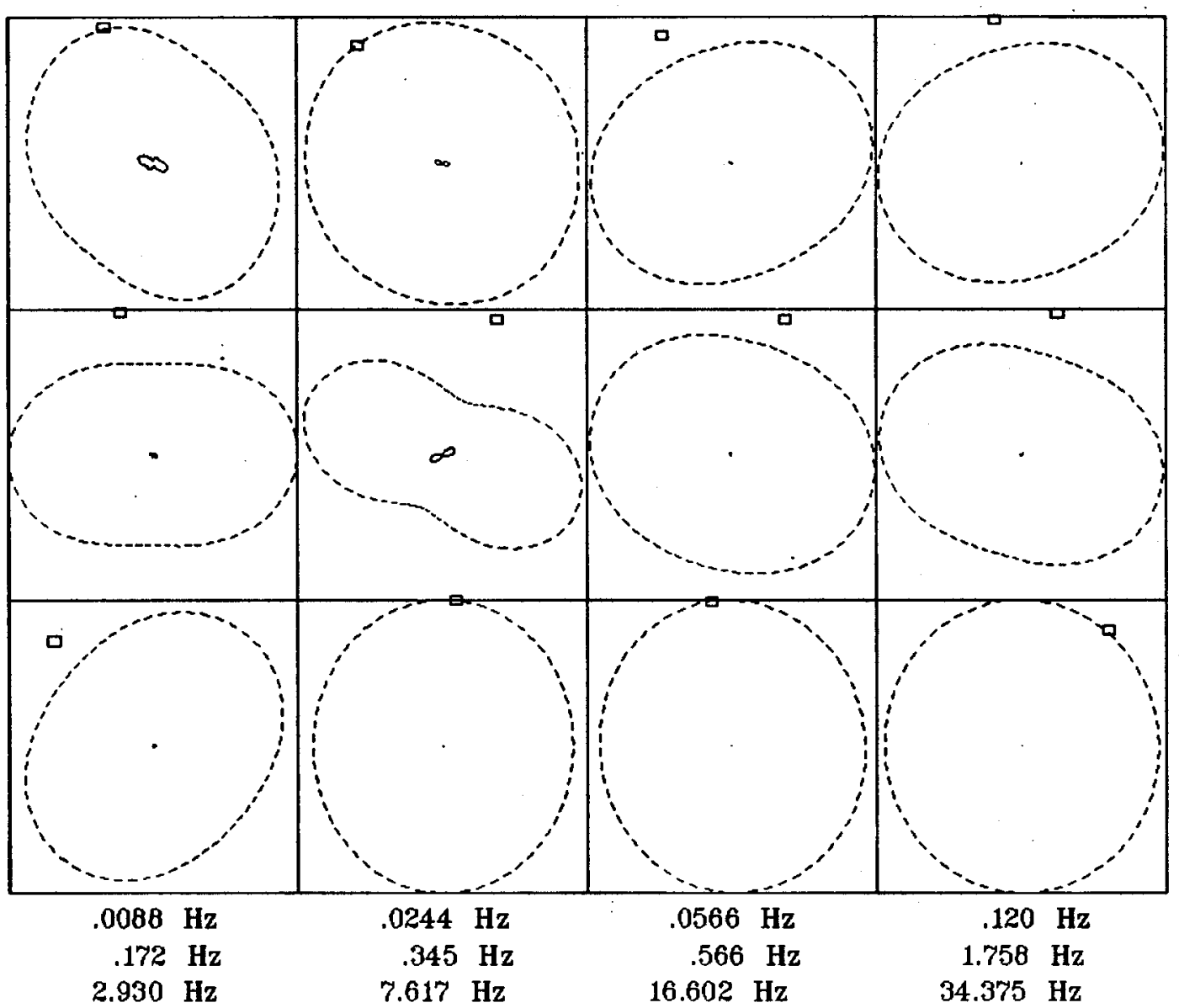

\section{Client:}

Remote: none

Acquired: 10:4 Jul 22, 2000

Survey Co:USGS
Rotation:

Filename: hr48c.avg

Channels: Ch1 Ch2 Ch3 Ch4 Ch5 Ch3 Ch4

Plotted: 11:05 Feb 06, 2001

< EMI - ElectroMagnetic Instruments > 
TIPPER MAGNITUDE

Osgood Mtns, NV 100K

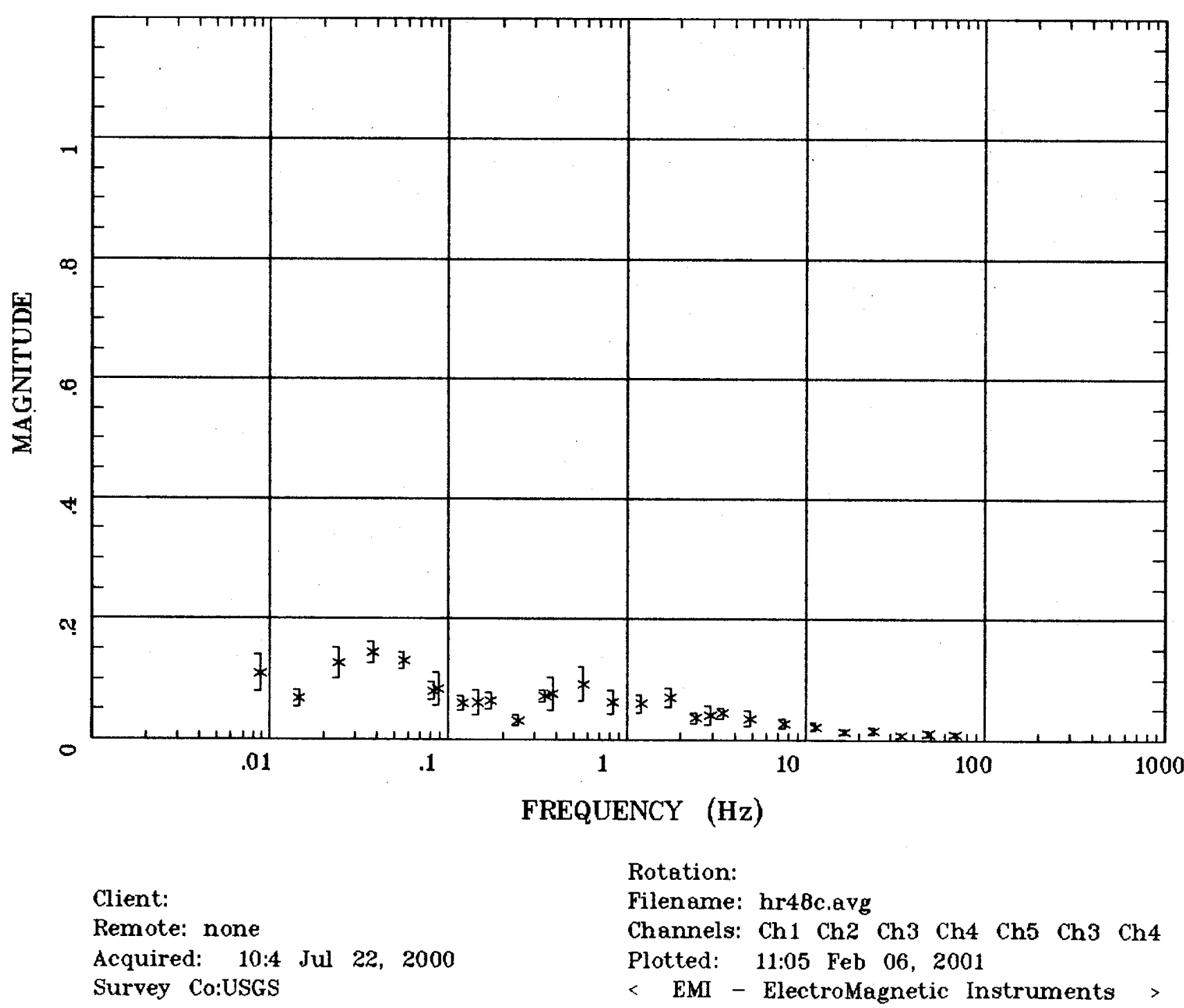




\section{Station 48}

TIPPER STRIKE

Osgood Mtns, NV 100K

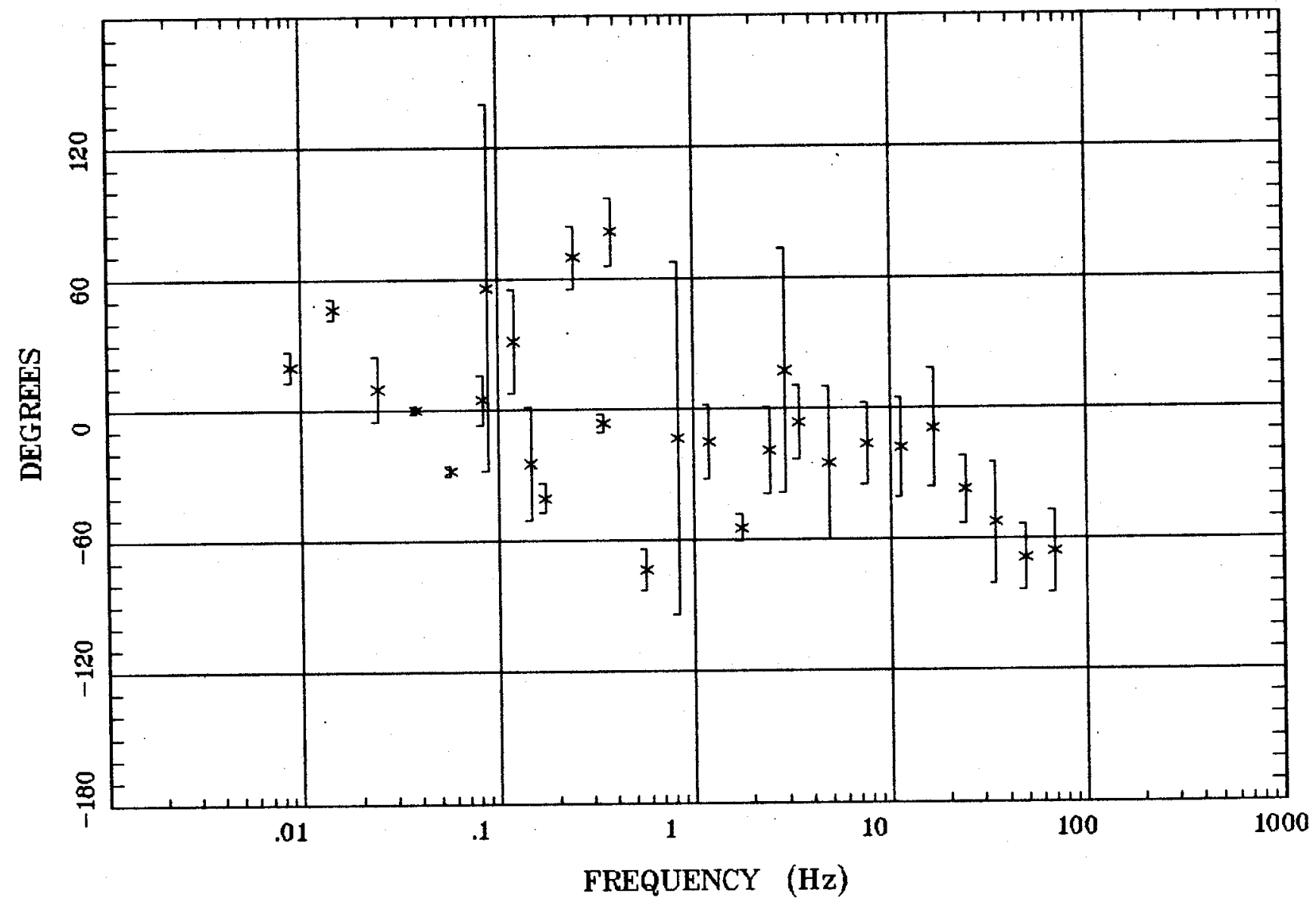

Client:

Remote: none

Rotation:

Acquired: 10:4 Jul 22, 2000

Survey Co:USGS

Filename: hr4bc.avg

Channels: Ch1 Ch2 Ch3 Ch4 Ch5 Ch3 Ch4

Plotted: 11:05 Feb 06, 2001

< EMI - ElectroMagnetic Instruments > 
HzHx.x Coh HzHy.o

Osgood Mtns, NV 100K

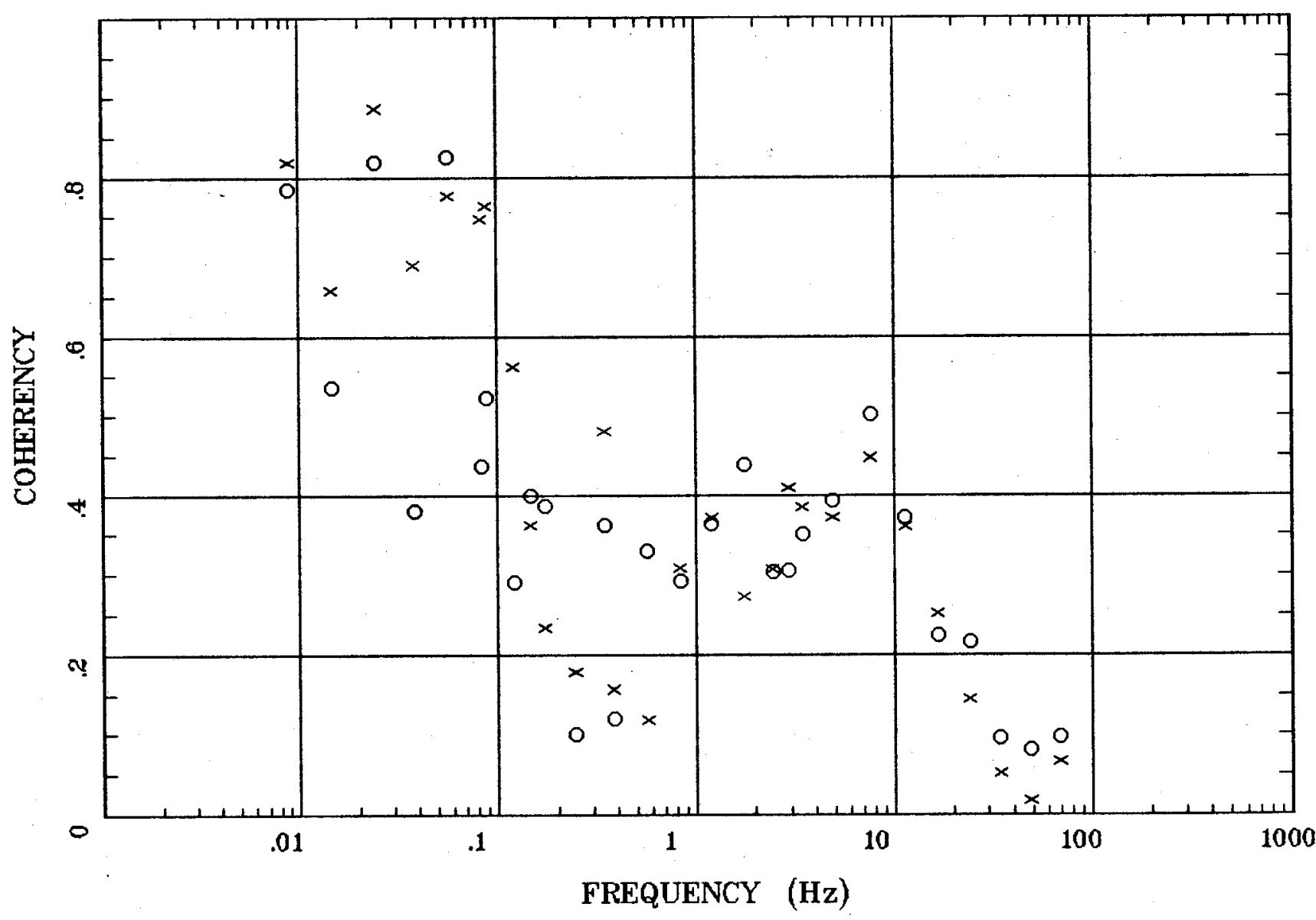

Client:

Remote: none

Acquired: 10:4 Jul 22, 2000

Survey Co:USGS
Rotation:

Filename: hr48c.avg

Channels: Ch1 Ch2 Ch3 Ch4 Ch5 Ch3 Ch4

Plotted: 11:05 Feb 06, 2001

$<$ EMI - ElectroMagnetic Instruments > 
Humboldt River Line 1

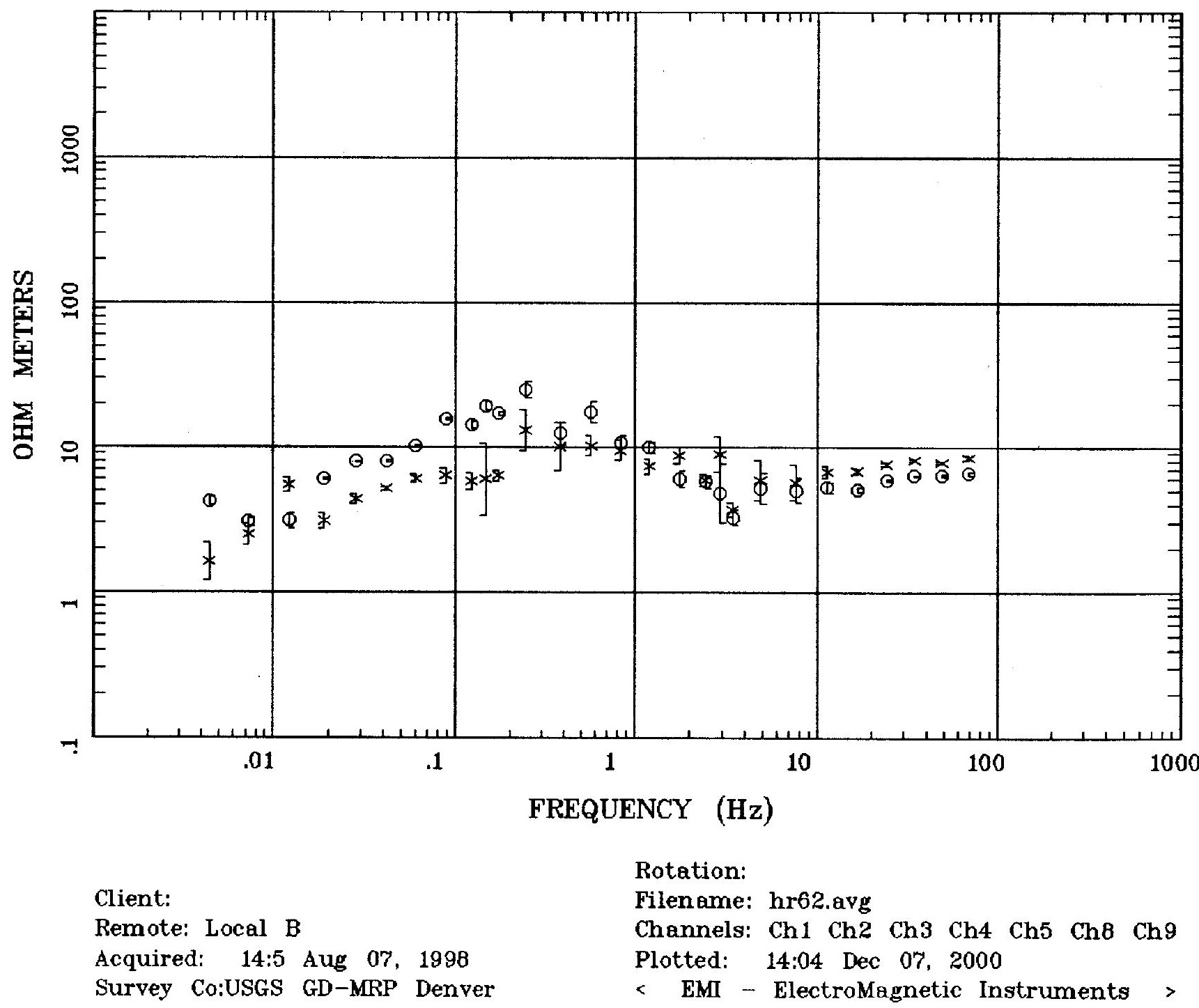


IMPEDANCE PHASE

Humboldt River Line 1

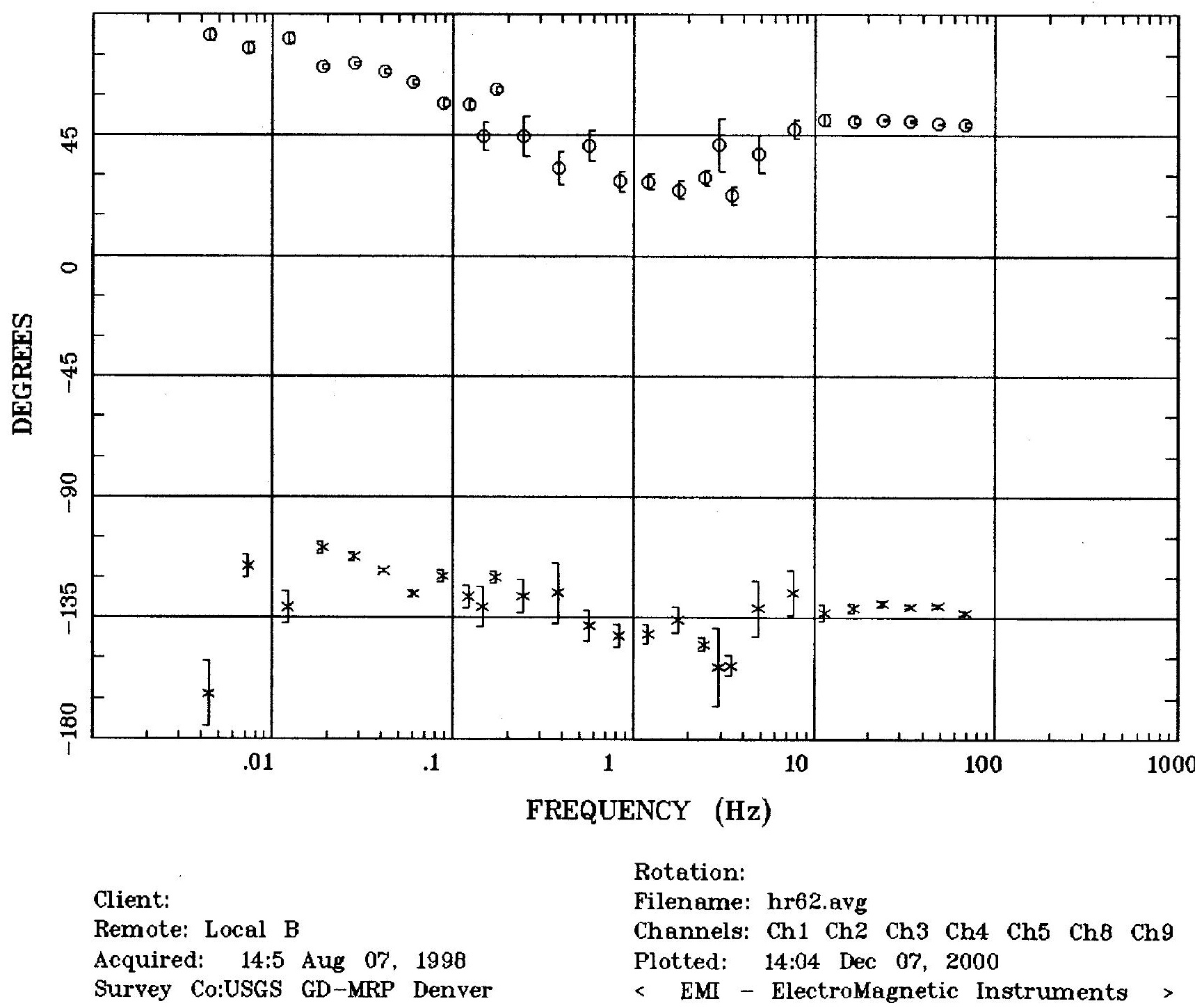




\section{Station 62}

Humboldt River Line 1

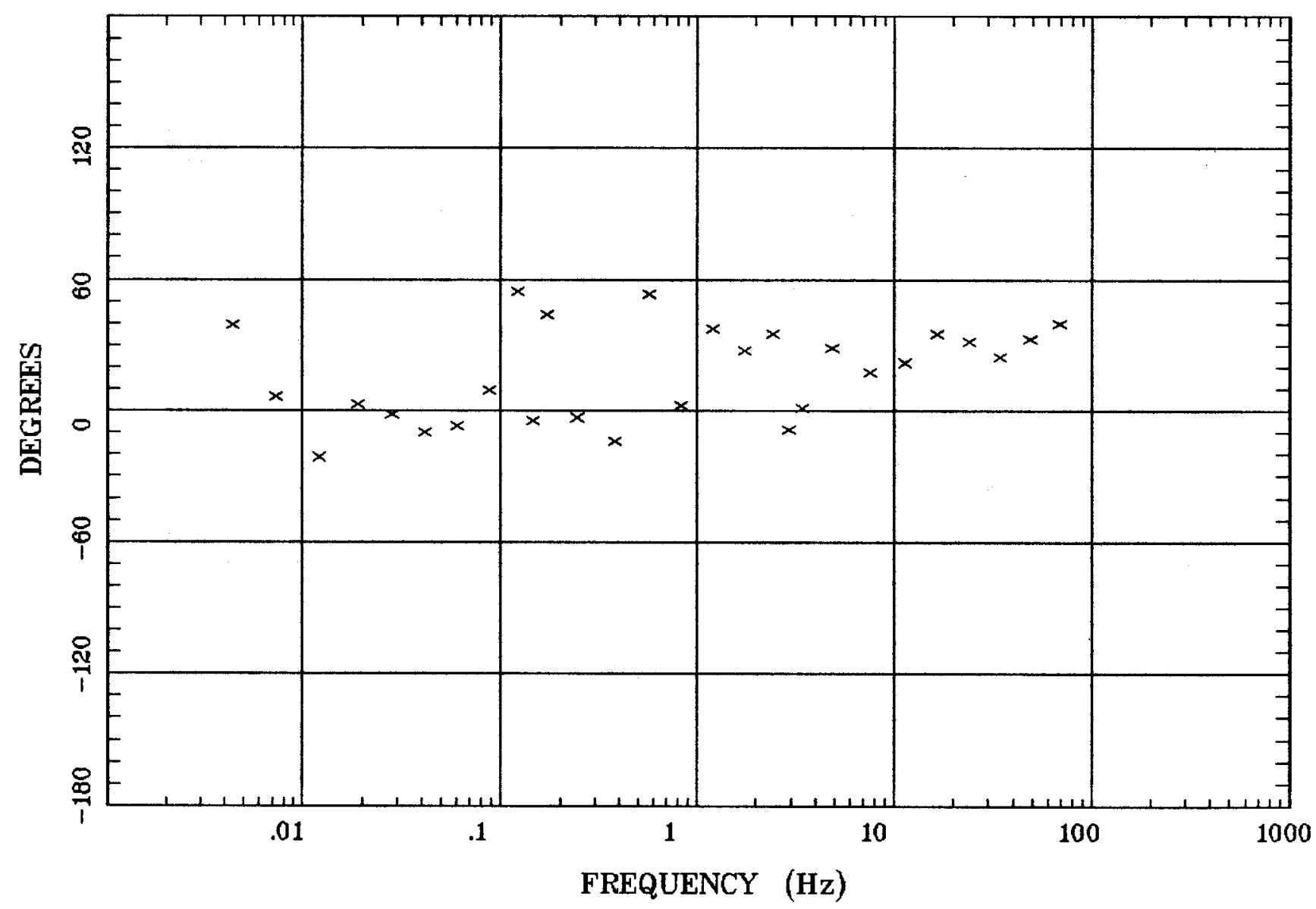

Client:

Remote: Local B

Acquired: 14:5 Aug 07, 1998 Survey Co:USGS GD-MRP Denver
Rotation:

Filename: hr62,avg

Channels: Ch1 Ch2 Ch3 Ch4 Ch5 Ch8 Ch9

Plotted: 14:04 Dec 07, 2000

$<$ EMI - ElectroMagnetic Instruments 
IMPEDANCE SKEW

Humboldt River Line 1

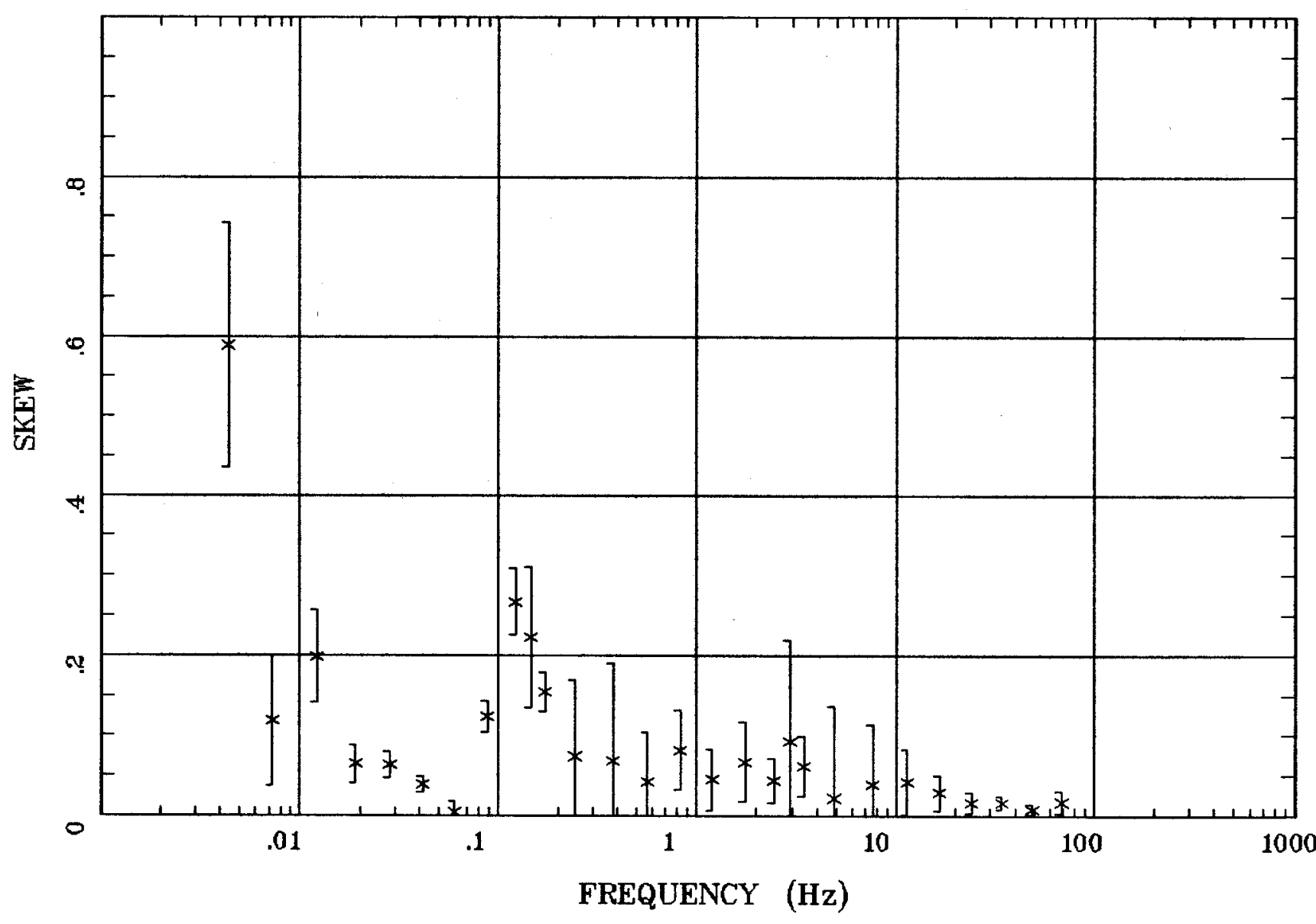

Client:

Remote: Local $B$

Acquired: 14:5 Aug 07, 1998

Survey Co:USGS GD-MRP Denver
Rotation:

Filename: hr62.avg

Channels: Ch1 Ch2 Ch3 Ch4 Ch5 ch8 Ch9

Plotted: 14:04 Dec 07, 2000

$<$ EMI - ElectroMagnetic Instruments > 
Station 62

E MULT Coh.

Humboldt River Line 1

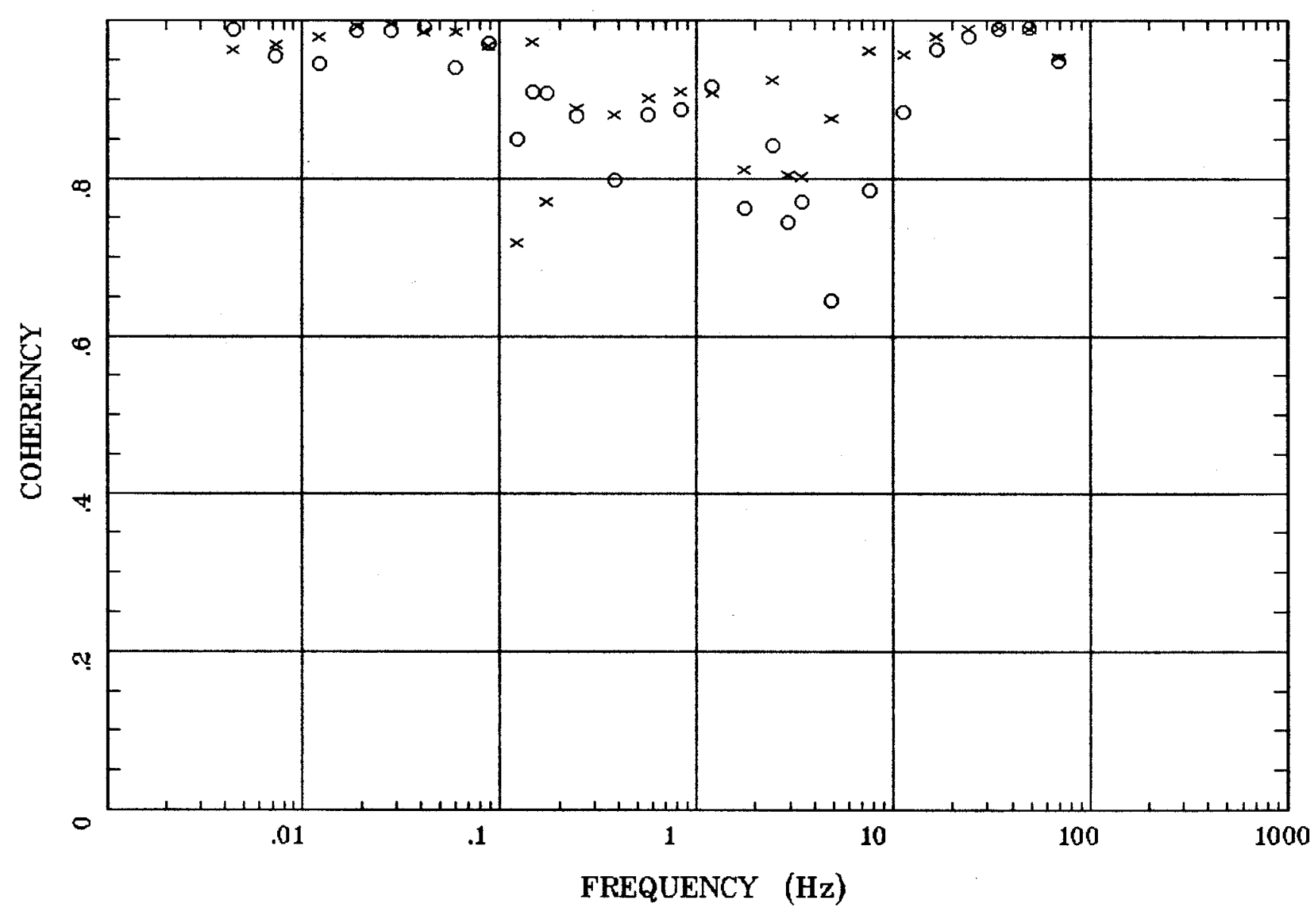

Client:

Remote: Local B

Acquired: 14:5 Aug 07, 1998 Survey Co:USGS GD-MRP Denver
Rotation:

Filename: hr62.avg

Channels: Ch1 Ch2 Ch3 Ch4 Ch5 Ch8 Ch9

Plotted: 14:05 Dec 07, 2000

< EMI - ElectroMagnetic Instruments 
Station 62

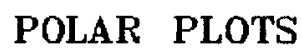

Humboldt River Line 1

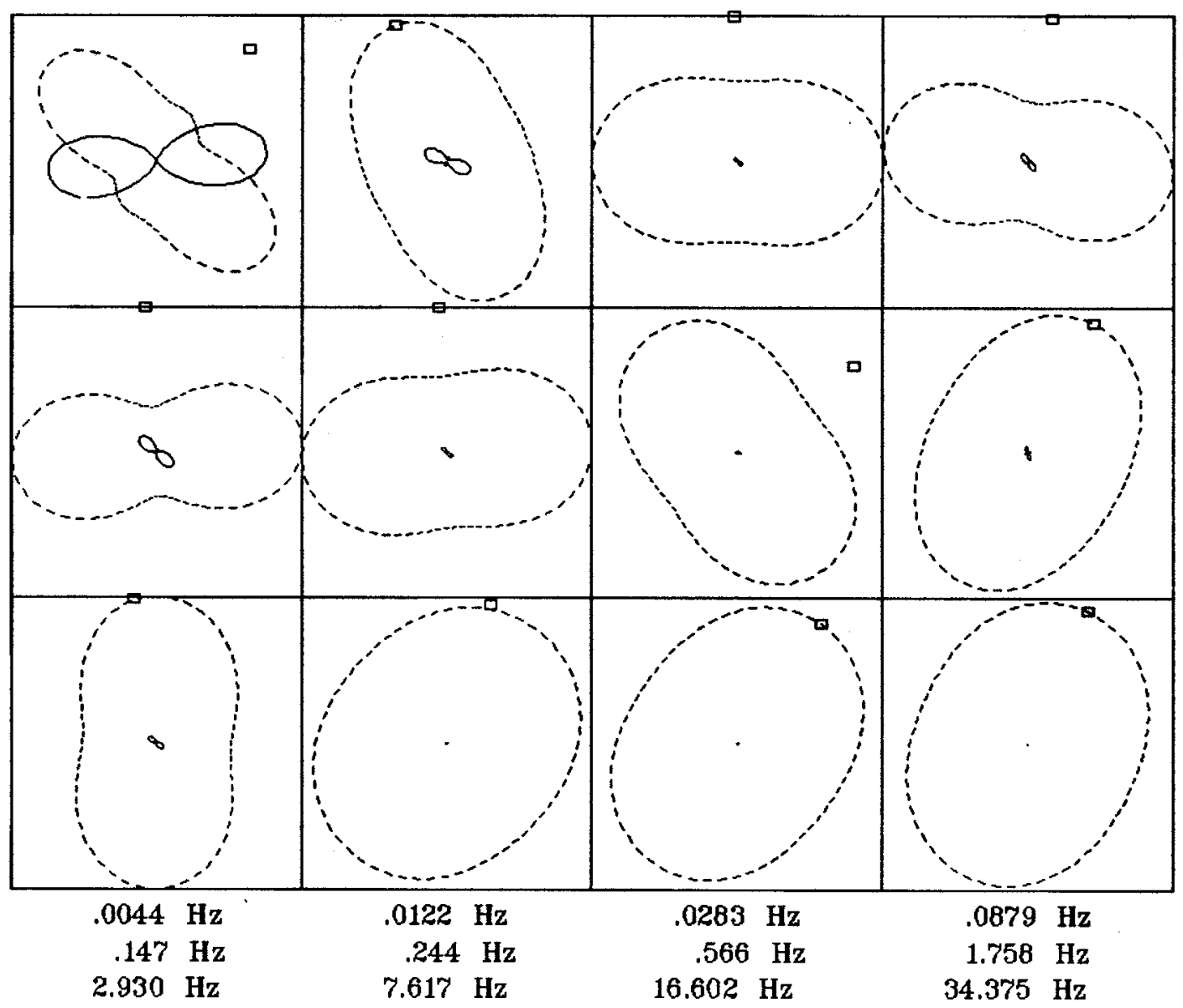

Client:

Remote: Local B

Acquired: 14:5 Aug 07, 1998 Survey Co:USGS GD-MRP Denver
Rotation:

Filename: hr62.avg

Channels: Ch1 Ch2 Ch3 Ch4 Ch5 Ch8 Ch9 Plotted: 14:05 Dec 07, 2000

$<$ EMI - ElectroMagnetic Instruments > 


\section{APPARENT RESISTIVITY}

Humboldt River Line 1

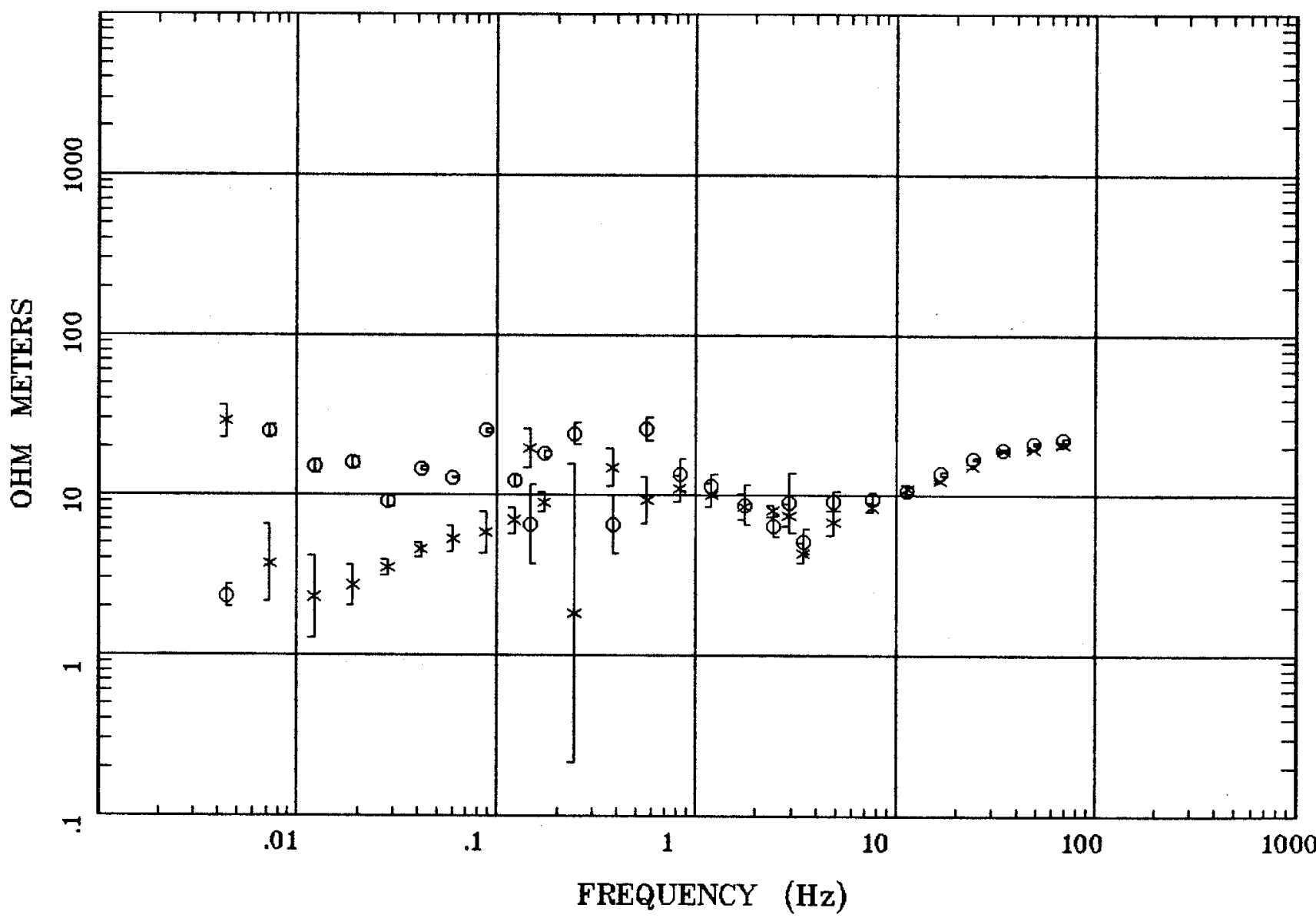

Client:

Remote: Local B

Acquired: 14:5 Aug 05, 1998

Survey Co:USGS GD-MRP Denver
Rotation:

Filename: hr45.avg

Channels: Ch1 Ch2 Ch3 Ch4 Ch5 Ch8 Ch9

Plotted: 13:53 Dec 07, 2000

$<$ EMI - ElectroMagnetic Instruments > 


\section{Station 45}

IMPEDANCE PHASE

Humboldt River Line 1

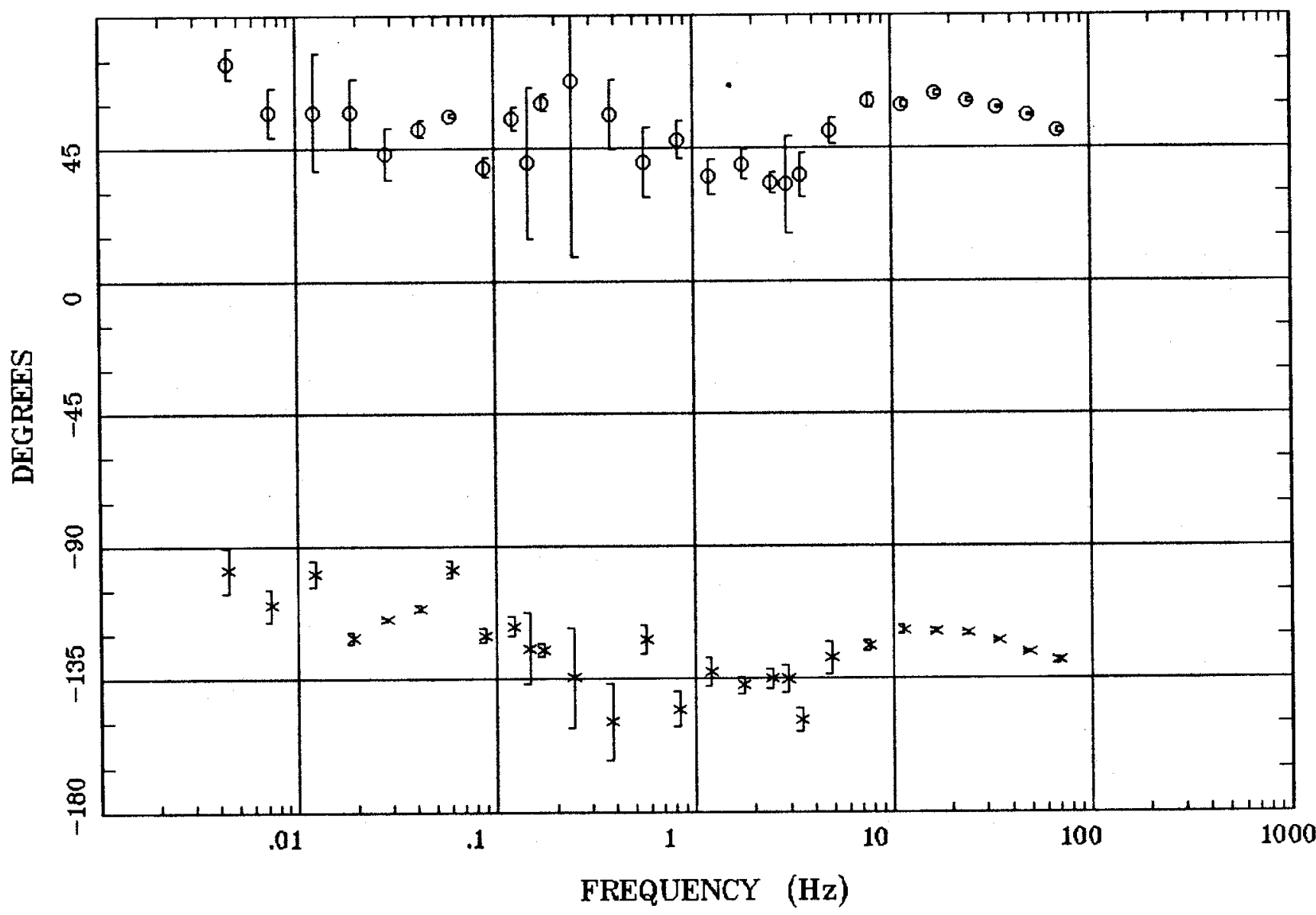

Client:

Remote: Local B

Acquired: 14:5 Aug 05, 1998 Survey Co:USGS GD-MRP Denver
Rotation:

Filename: hr45.avg

Channels: Ch1 Ch2 Ch3 Ch4 Ch5 Ch8 Ch9

Plotted: 13:53 Dec 07, 2000

$<$ EMI - ElectroMagnetic Instruments > 
Humboldt River Line 1

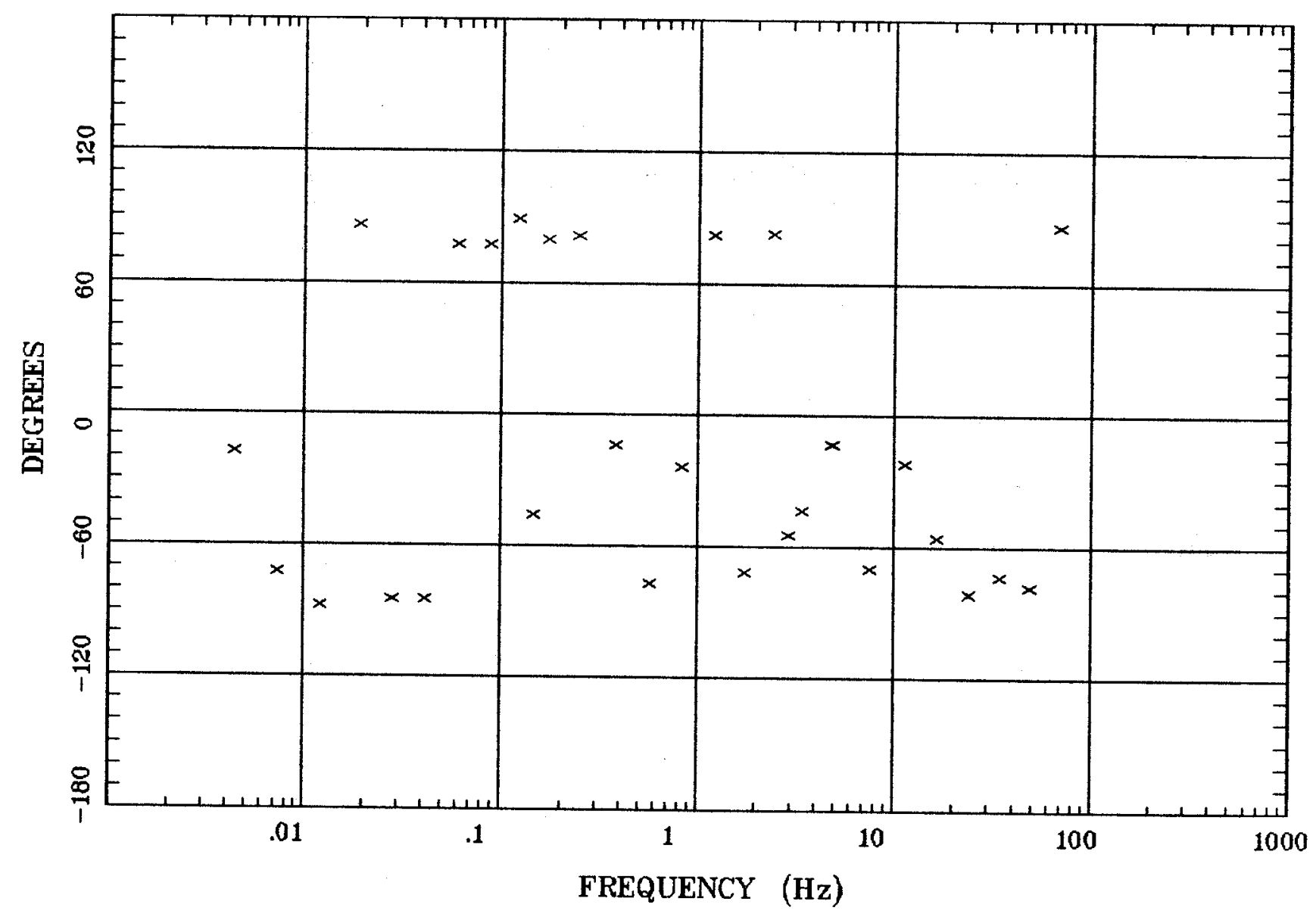

Client:

Remate: Local $B$

Acquired: 14:5 Aug 05, 1998

Survey Co:USGS GD-MRP Denver
Rotation:

Filename: hr45.avg

Channels: Ch1 Ch2 Ch3 Ch4 Ch5 Ch8 Ch9

Plotted: 13:53 Dec 07, 2000

< EMI - ElectroMagnetic Instruments > 
Humboldt River Line 1

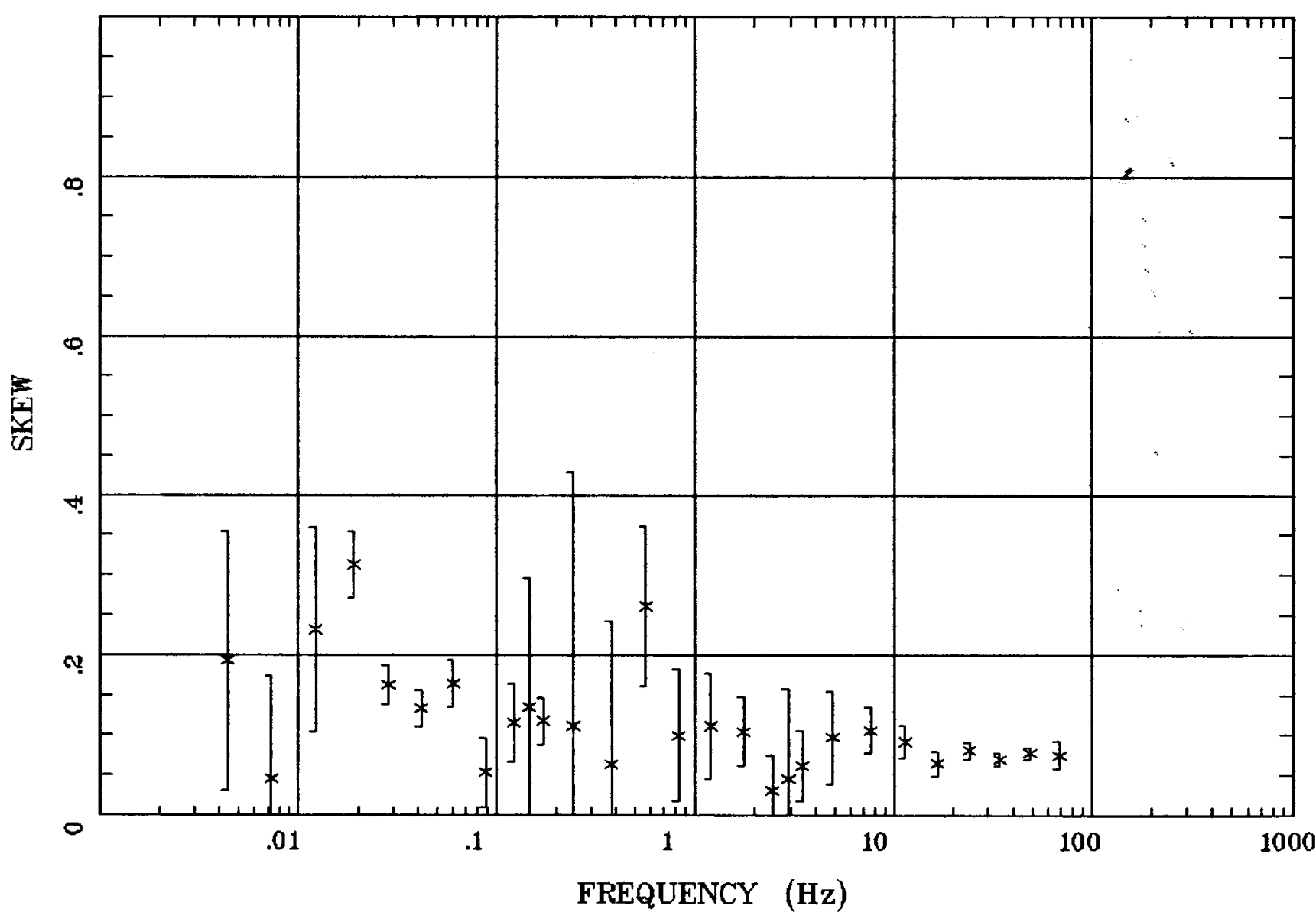

Client:

Remote: Local B

Acquired: 14:5 Aug 05, 1998

Survey Co:USGS GD-MRP Denver
Rotation:

Filename: hr45.avg

Channels: Ch1 Ch2 Ch3 Ch4 Ch5 Ch8 Ch9

Plotted: 13:53 Dec 07, 2000

< EMI - ElectroMagnetic Instruments > 
E MULT Coh.

Humboldt River Line 1

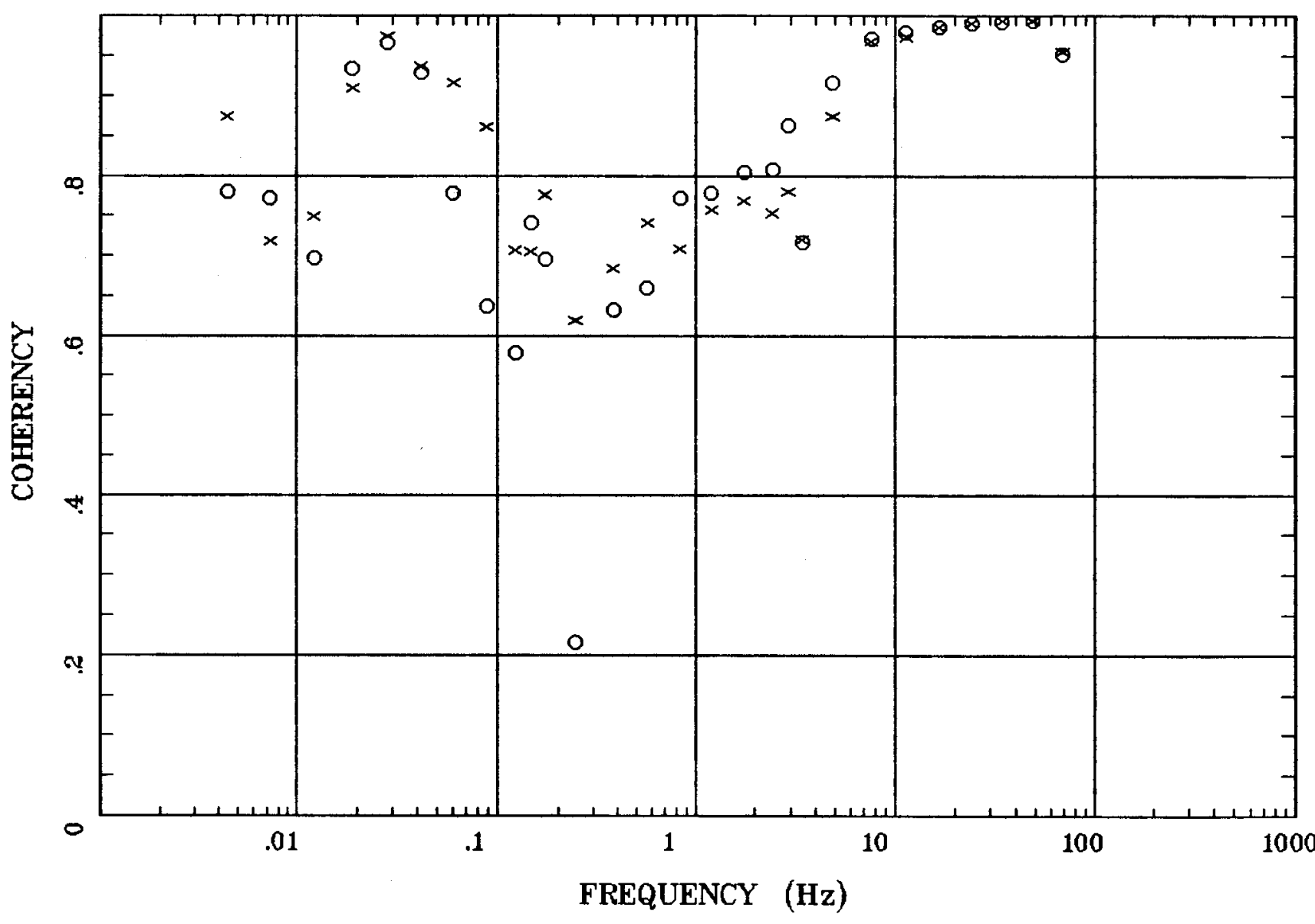

Client:

Remote: Local B

Acquired: 14:5 Aug 05, 1998

Survey Co:USGS GD-MRP Denver

Rotation:

Filename: hr45.avg

Channels: Ch1 Ch2 Ch3 Ch4 Ch5 Ch8 Ch9

Plotted: 13:53 Dec 07, 2000

< EMI - ElectroMagnetic Instruments 
Humboldt River Line 1

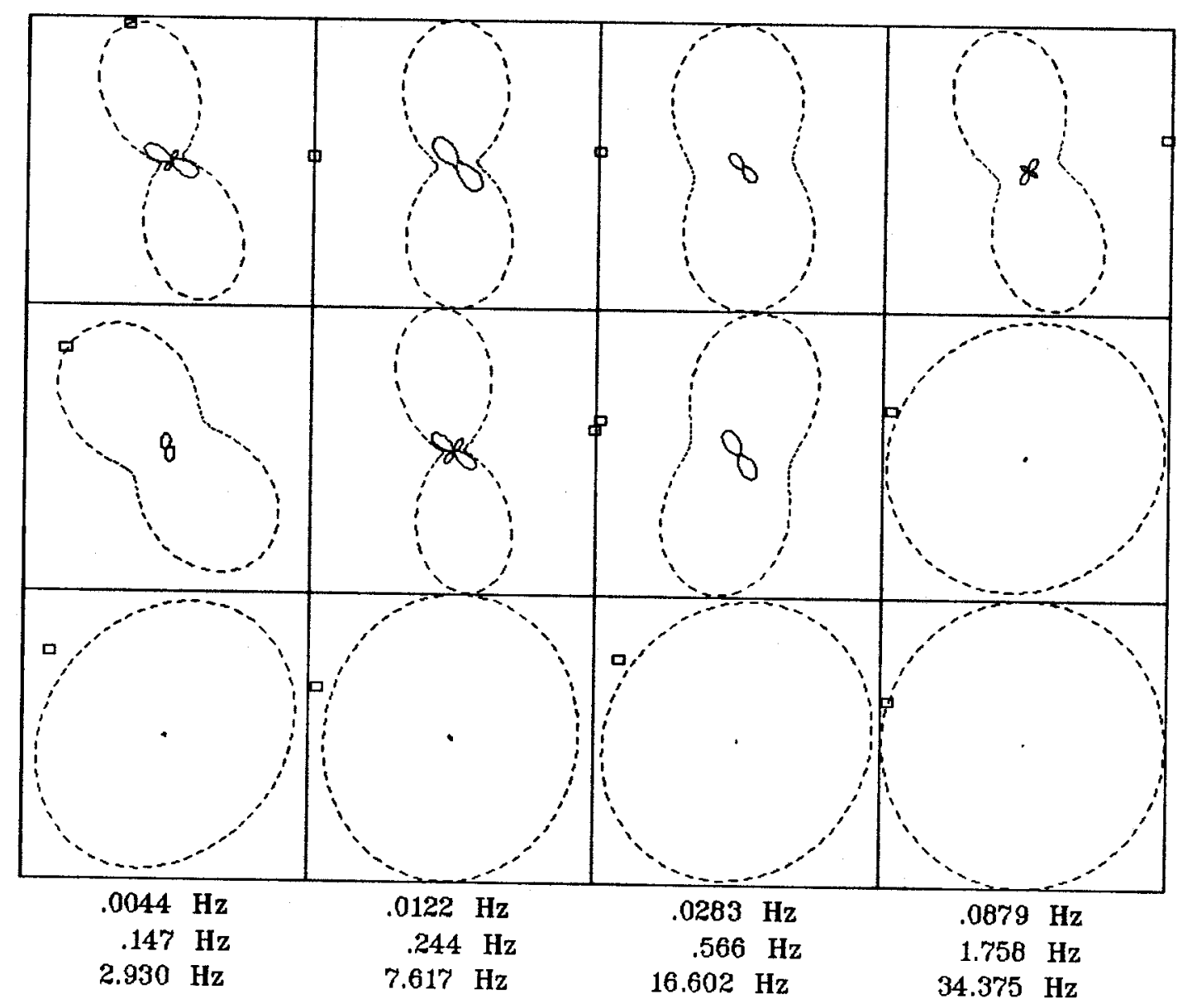

Client:

Remote: Local B

Acquired: 14:5 Aug 05, 1998 Survey Co:USGS GD-MRP Denver
Rotation:

Filename: hr45.avg

Channels: Ch1 Ch2 Ch3 Ch4 Ch5 Ch8 Ch9

Plotted: 13:53 Dec 07, 2000

< EMI - ElectroMagnetic Instruments > 
APPARENT RESISTIVITY

Humboldt River Line 1



Client:

Remote: Local B

Acquired: 11:0 Aug 06, 1998

Survey Co:USGS GD-MRP Denver
Rotation:

Filename: hr60.avg

Channels: Ch1 Ch2 Ch3 Ch4 Ch5 Ch8 Ch9

Plotted: 14:03 Dec 07, 2000

< EMI - ElectroMagnetic Instruments > 
Station 60

IMPEDANCE PHASE

Humboldt River Line 1

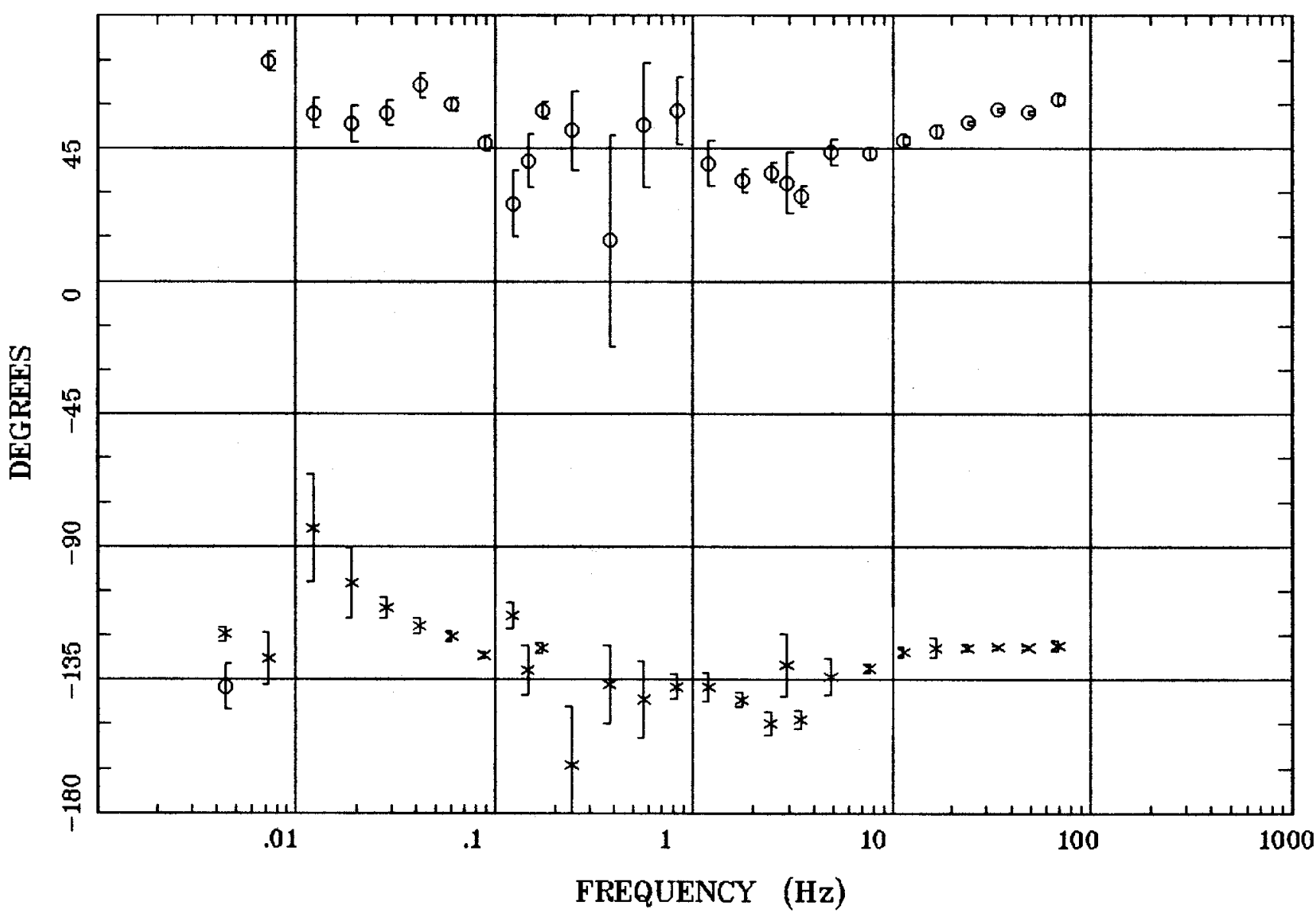

Client:

Remote: Local B

Acquired: 11:0 Aug 06, 1998

Filename: hr60.avg

Survey Co:USGS GD-MRP Denver

Channels: Ch1 Ch2 Ch3 Ch4 Ch5 Ch8 Ch9

Plotted: 14:03 Dec 07, 2000

< EMI - ElectroMagnetic Instruments > 


\section{ROTATION ANGLE}

Humboldt River Line 1

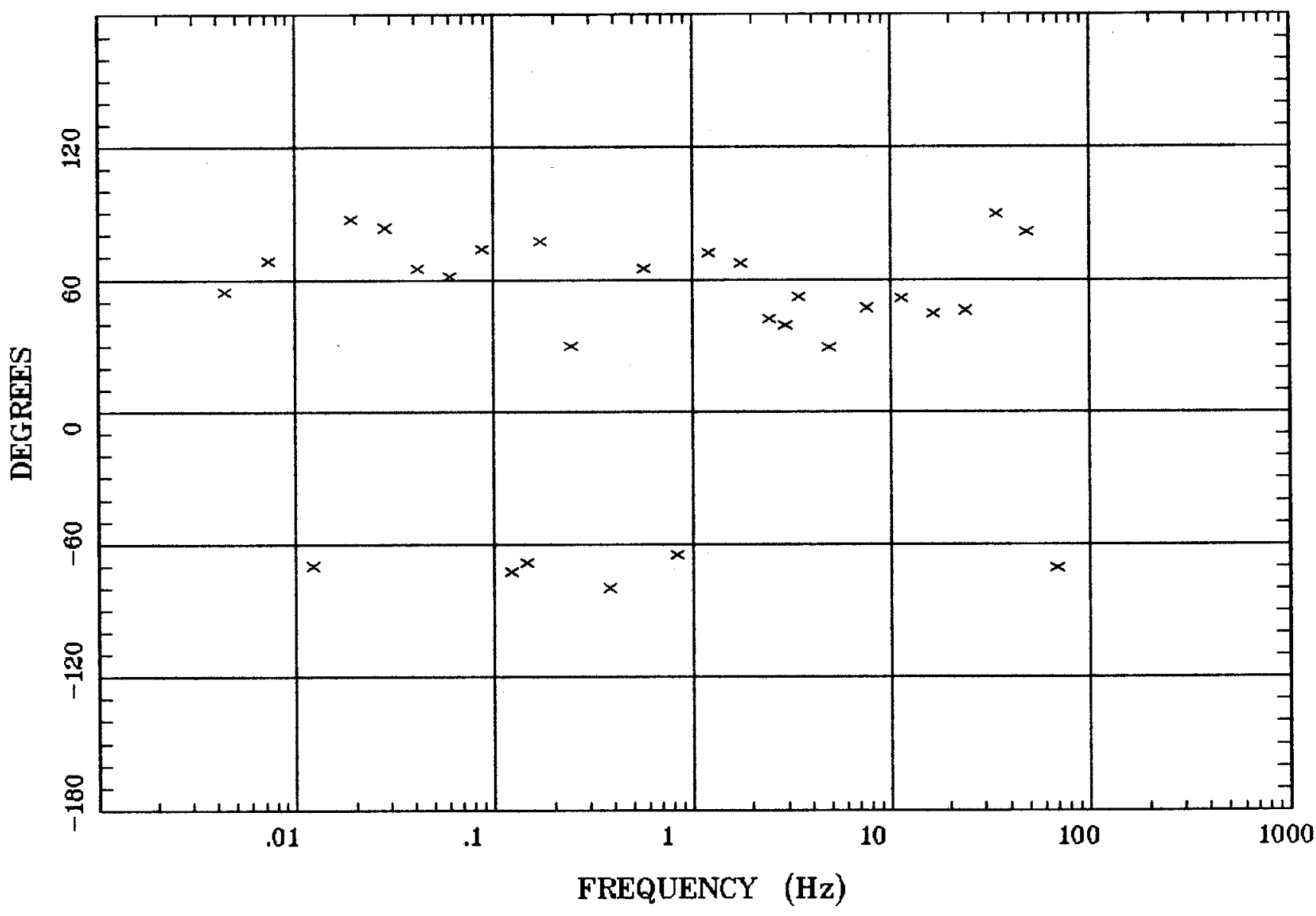

Client:

Remote: Local B

Acquired: 11:0 Aug 06, 1998 Survey Co:USGS GD-MRP Denver
Rotation:

Filename: hr60.avg

Channels: Ch1 Ch2 Ch3 Ch4 Ch5 Ch8 Ch9 Platted: 14:03 Dec 07, 2000

< EMI - ElectroMagnetic Instruments > 
IMPEDANCE SKEW

Humboldt River Line 1

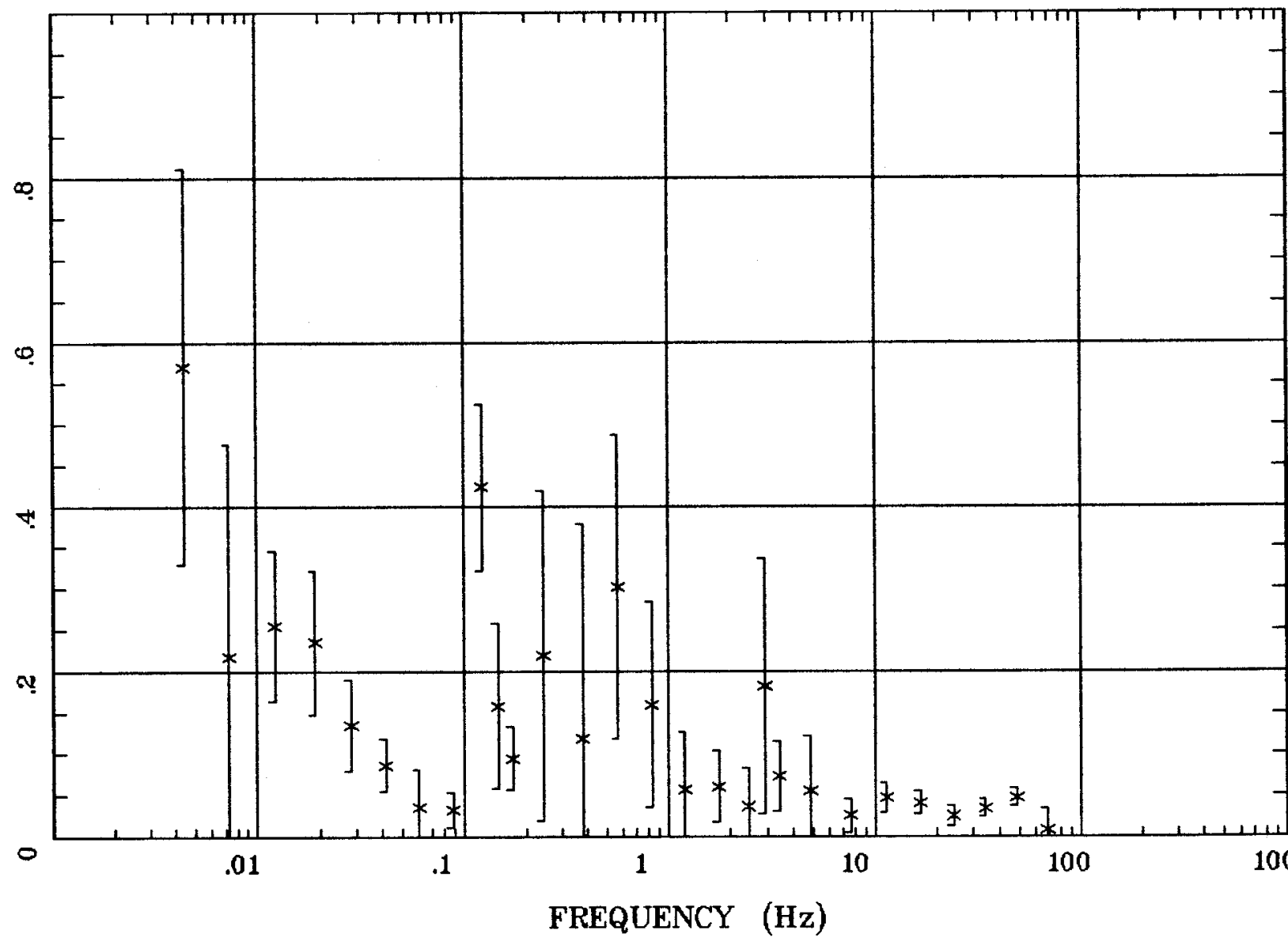

Client:

Remote: Local $B$

Acquired: 11:0 Aug 06, 1998

Survey Co:USGS GD-MRP Denver
Rotation:

Filename: hr60.avg

Channels: Ch1 Ch2 Ch3 Ch4 Ch5 Ch8 Ch9 Plotted: 14:03 Dec 07, 2000

$<$ EMI - ElectroMagnetic Instruments 
Station 60

E MULT Coh.

Humboldt River Line 1



Client:

Rotation:

Remote: Local B

Acquired: 11:0 Aug 06, 1998

Survey Co:USGS GD-MRP Denver

Filename: hr60,avg

Channels: Ch1 Ch2 Ch3 Ch4 Ch5 Ch8 Ch9

Plotted: 14:03 Dec 07, 2000

< EMI - ElectroMagnetic Instruments > 
Humboldt River Line 1

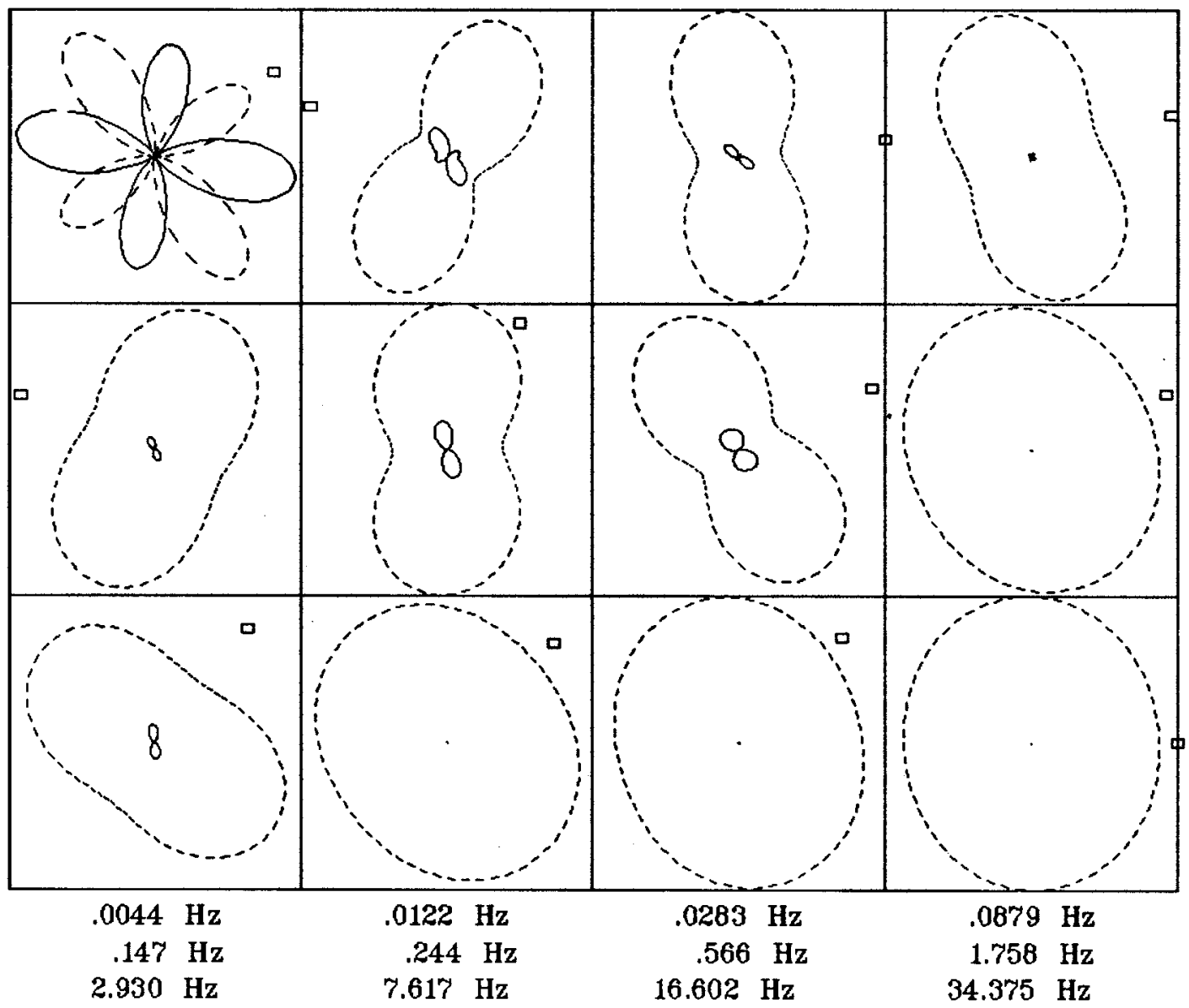

Client:

Remote: Local B

Acquired: 11:0 Aug 06, 1998 Survey Co:USGS GD-MRP Denver
Rotation:

Filename: hr60.avg

Channels: Ch1 Ch2 Ch3 Ch4 Ch5 Ch8 Ch9 Plotted: 14:03 Dec 07, 2000

< EMI - ElectroMagnetic Instruments > 
Station 43

APPARENT RESISTIVITY

Humboldt River Line 1

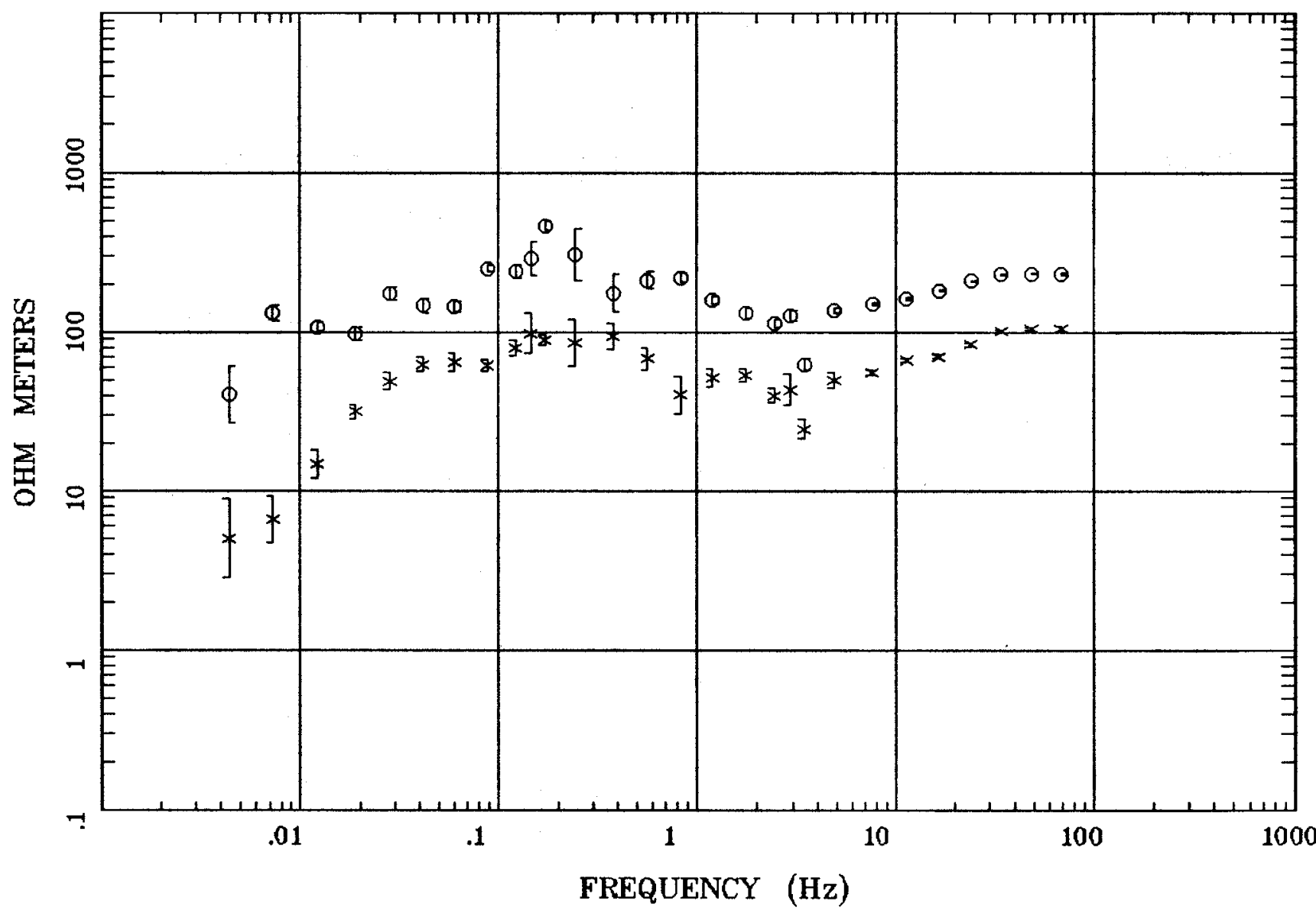

Client:

Remote: Local B

Acquired: 10:2 Aug 05, 1998

Survey Co:USGS GD-MRP Denver
Rotation:

Filename: hr43.avg

Channels: Ch1 Ch2 Ch3 Ch4 Ch5 Ch8 Ch9

Plotted: 13:52 Dec 07, 2000

<EMI - ElectroMagnetic Instruments > 


\section{Station $\mathbf{4 3}$}

IMPEDANCE PHASE

Humboldt River Line 1

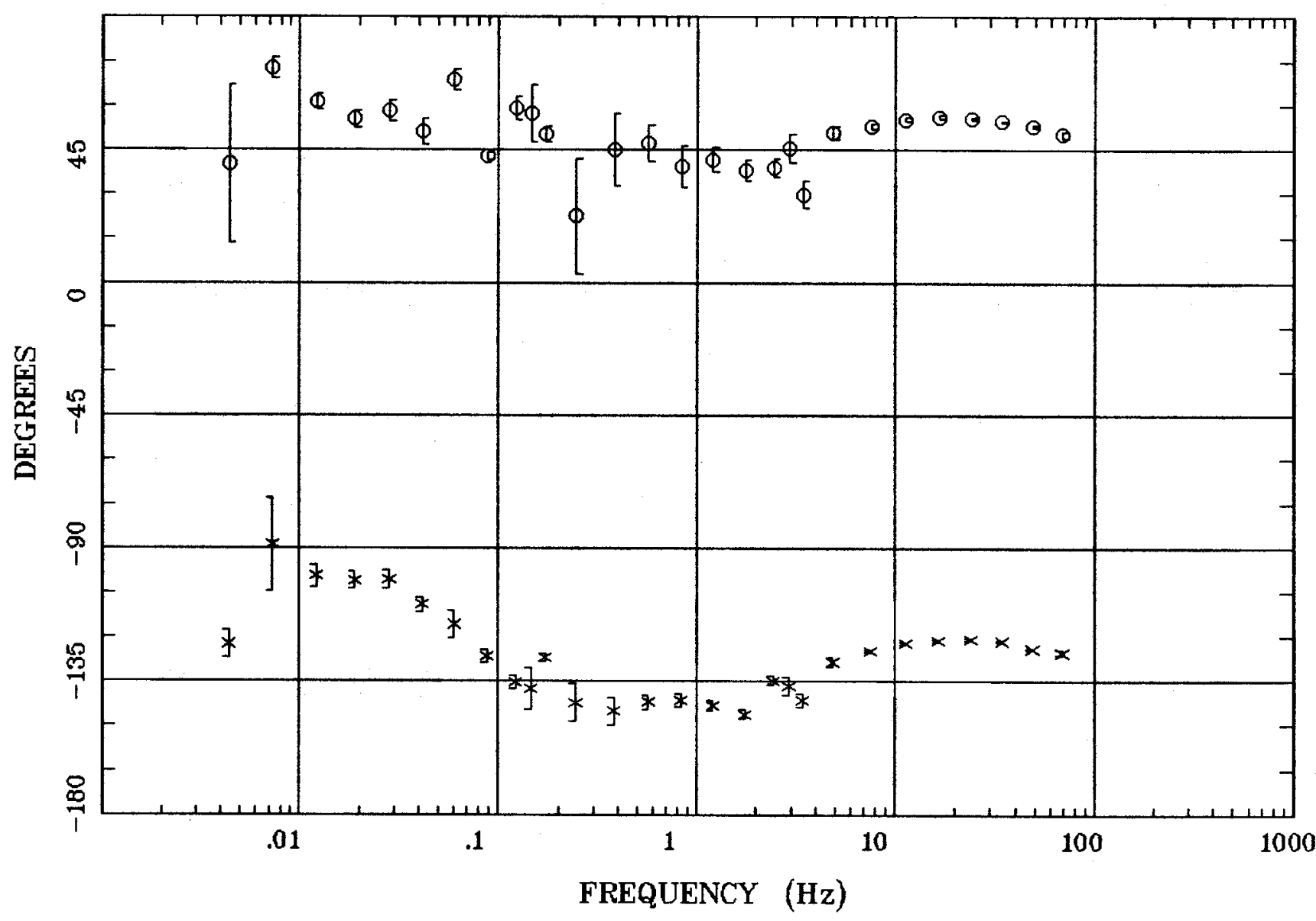

Client:

Remote: Local B

Acquired: 10:2 Aug 05, 1998

Survey Co:USGS GD-MRP Denver
Rotation:

Filename: hr43.avg

Channels: Ch1 Ch2 Ch3 Ch4 Ch5 Ch8 Ch9

Plotted: 13:52 Dec 07, 2000

$<$ EMI - ElectroMagnetic Instruments > 
Humboldt River Line 1

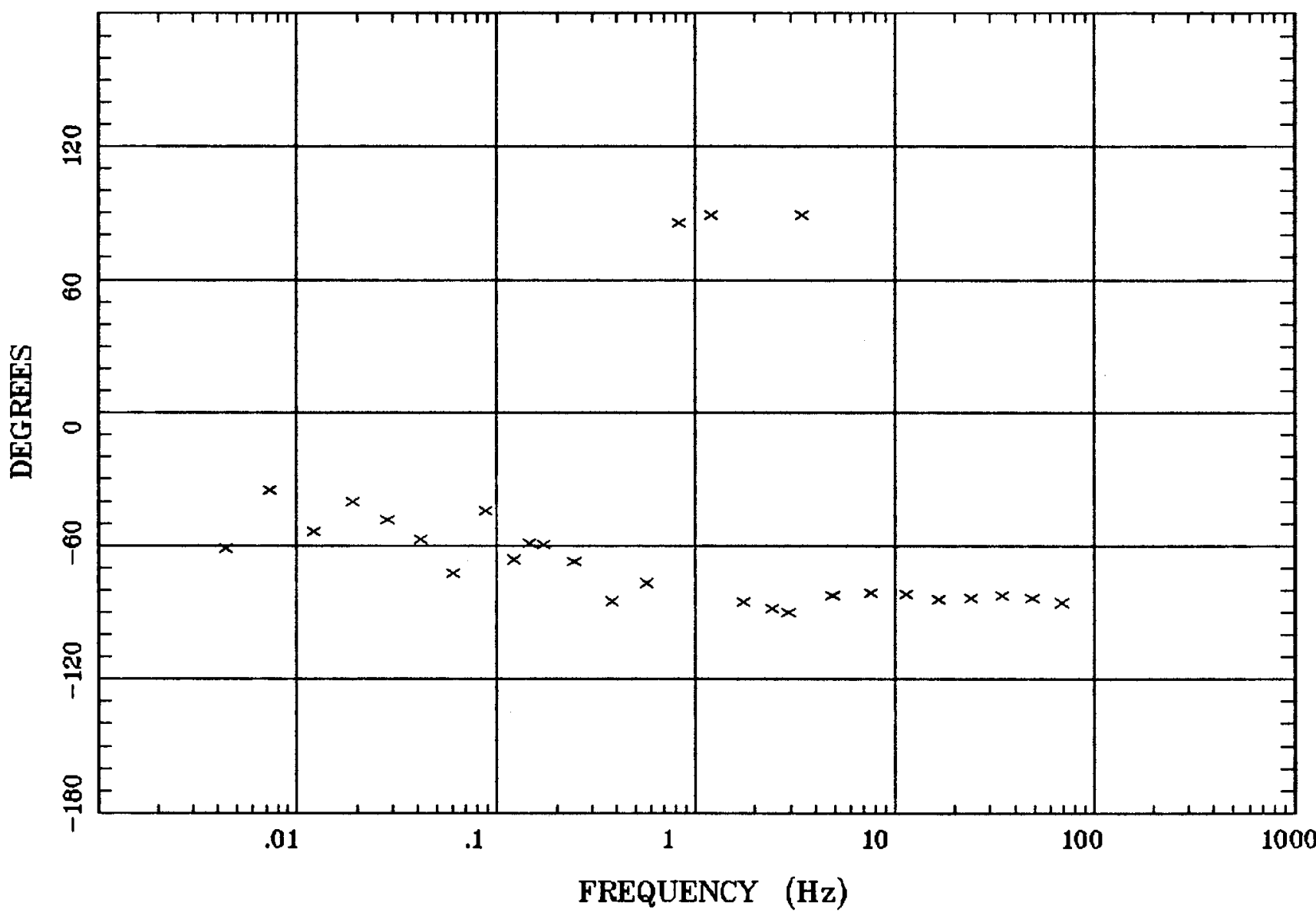

Client:

Remote: Local B

Acquired: 10:2 Aug 05, 1998

Survey Co:USGS GD-MRP Denver

Rotation:

Filename: hr43.avg

Channels: Ch1 Ch2 Ch3 Ch4 Ch5 Ch8 Ch9

Plotted: 13:52 Dec 07, 2000

< EMI - ElectroMagnetic Instruments > 
Station 43

IMPEDANCE SKEW

Humboldt River Line 1

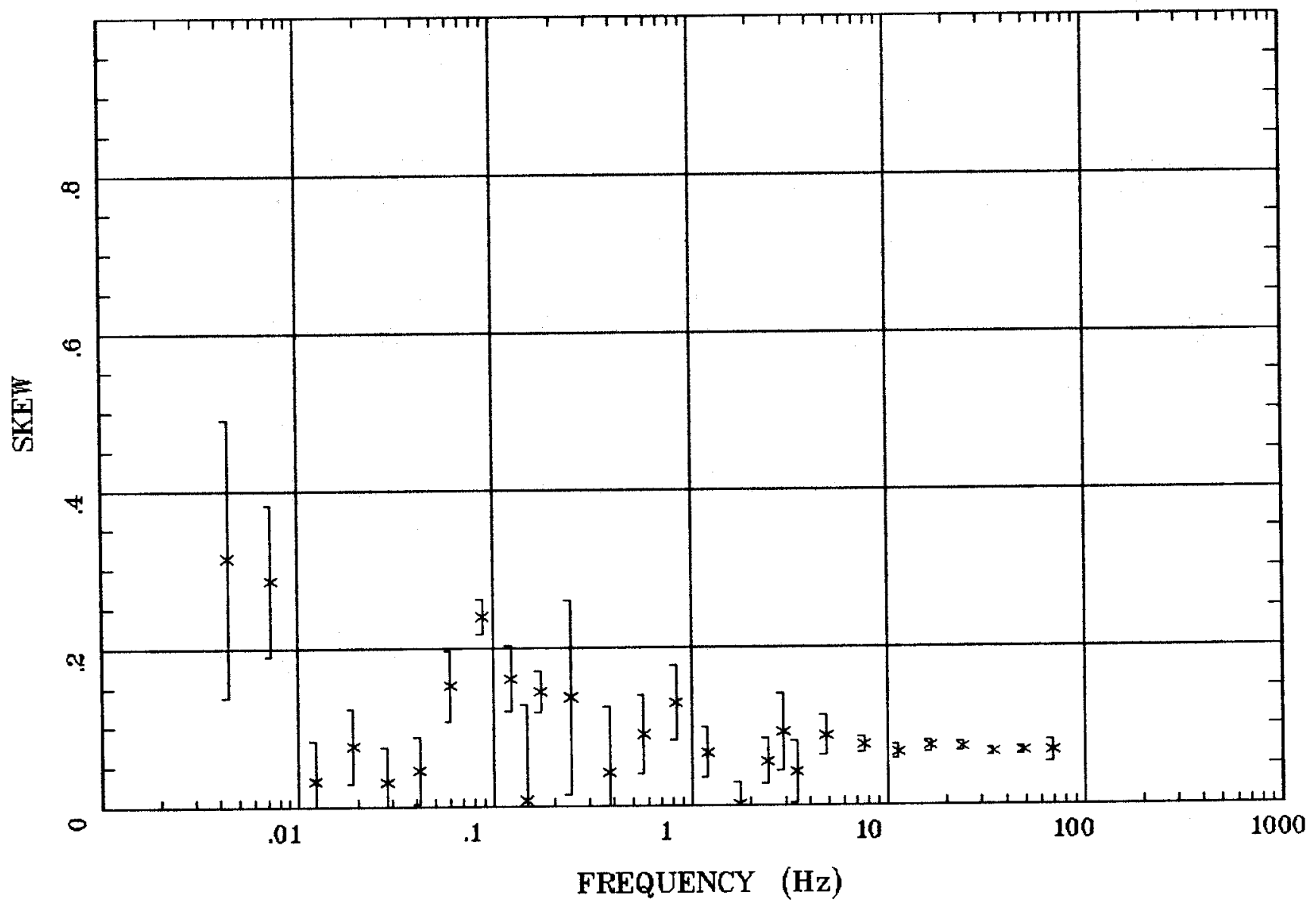

Client:

Remote: Local B

Acquired: 10:2 Aug 05, 1998

Survey Co:USGS GD-MRP Denver

Rotation:

Filename: hr43,avg

Channels: Ch1 Ch2 Ch3 Ch4 Ch5 Ch8 Ch9

Plotted: 13:52 Dec 07, 2000

< EMI - ElectroMagnetic Instruments > 
Station 43

E MULT Coh.

Humboldt River Line 1

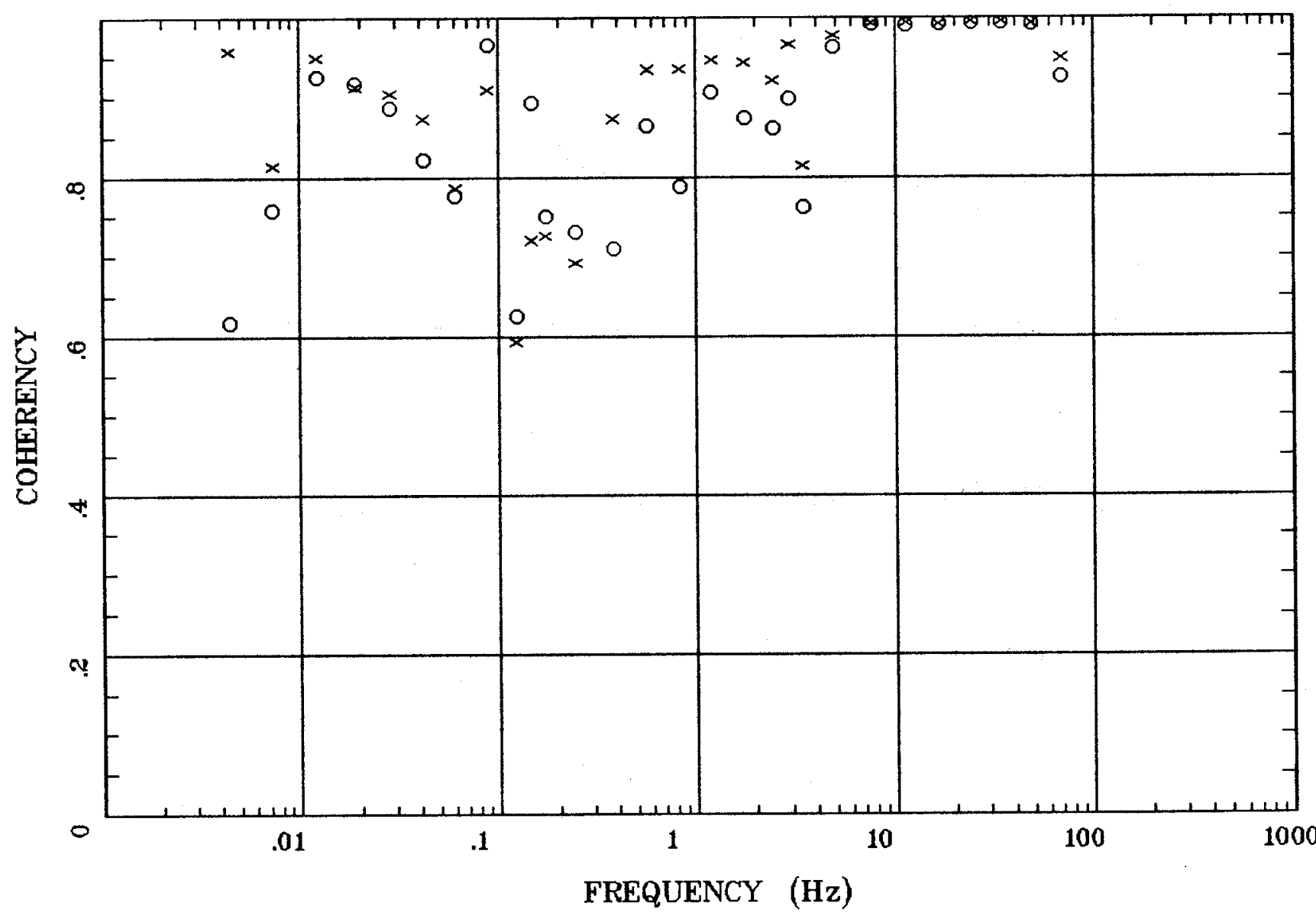

Client:

Remote: Local B

Acquired: 10:2 Aug 05, 1998 Survey Co:USGS GD-MRP Denver
Rotation:

Filename: hr43.avg

Channels: Ch1 Ch2 Ch3 Ch4 Ch5 Ch8 Ch9 Plotted: 13:52 Dec 07, 2000

$<$ EMI - ElectroMagnetic Instruments 


\section{POLAR PLOTS}

Humboldt River Line 1

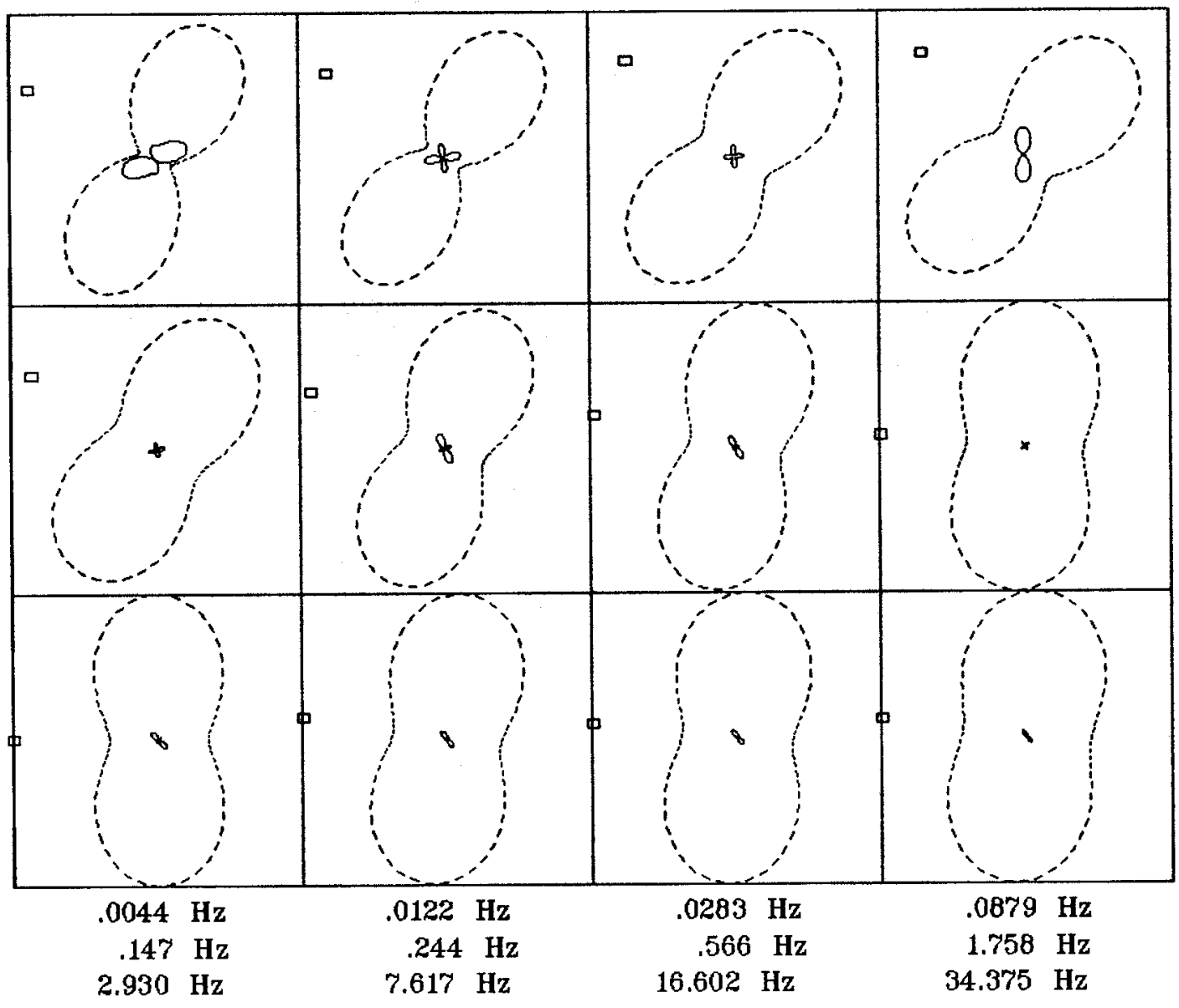

Rotation:

Client:

Remote: Local B

Acquired: 10:2 Aug 05, 1998

Filename: hr43.avg

Channels: Ch1 Ch2 Ch3 Ch4 Ch5 Ch8 Ch9

Plotted: 13:52 Dec 07, 2000

Survey Co:USGS GD-MRP Denver

< EMI - ElectroMagnetic Instruments 





Station 42

IMPEDANCE PHASE

Humboldt River Line 1

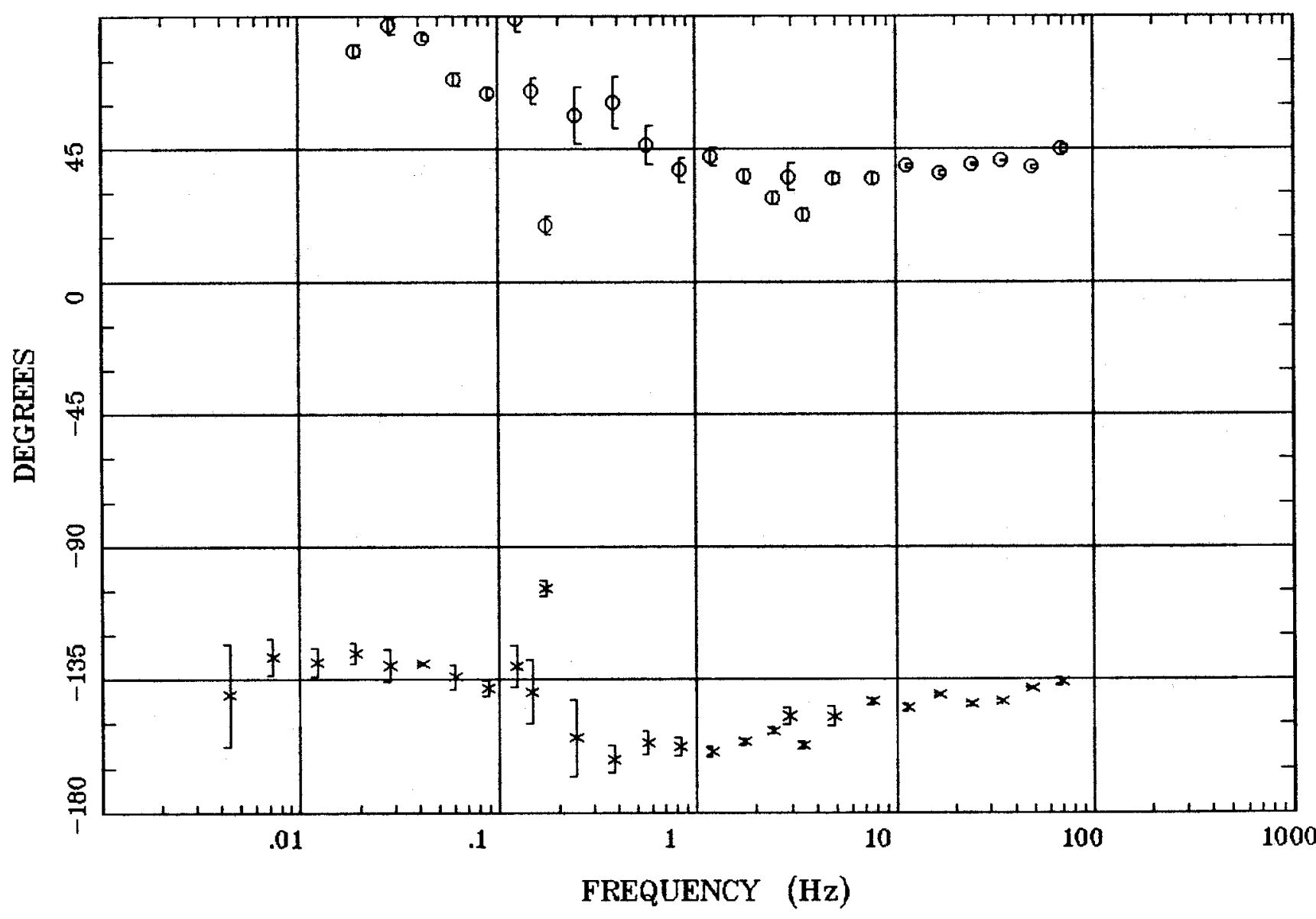

Client:

Remote: Local $B$

Acquired: 13:4 Aug 04, 1998

Survey Co:USGS GD-MRP Denver

Rotation:

Filename: hr42.avg

Channels: Ch1 Ch2 Ch3 Ch4 Ch5 Ch8 Ch9

Plotted: 13:50 Dec 07, 2000

< EMI - ElectroMagnetic Instruments 


\section{Station 42}

ROTATION ANGLE

Humboldt River Line 1

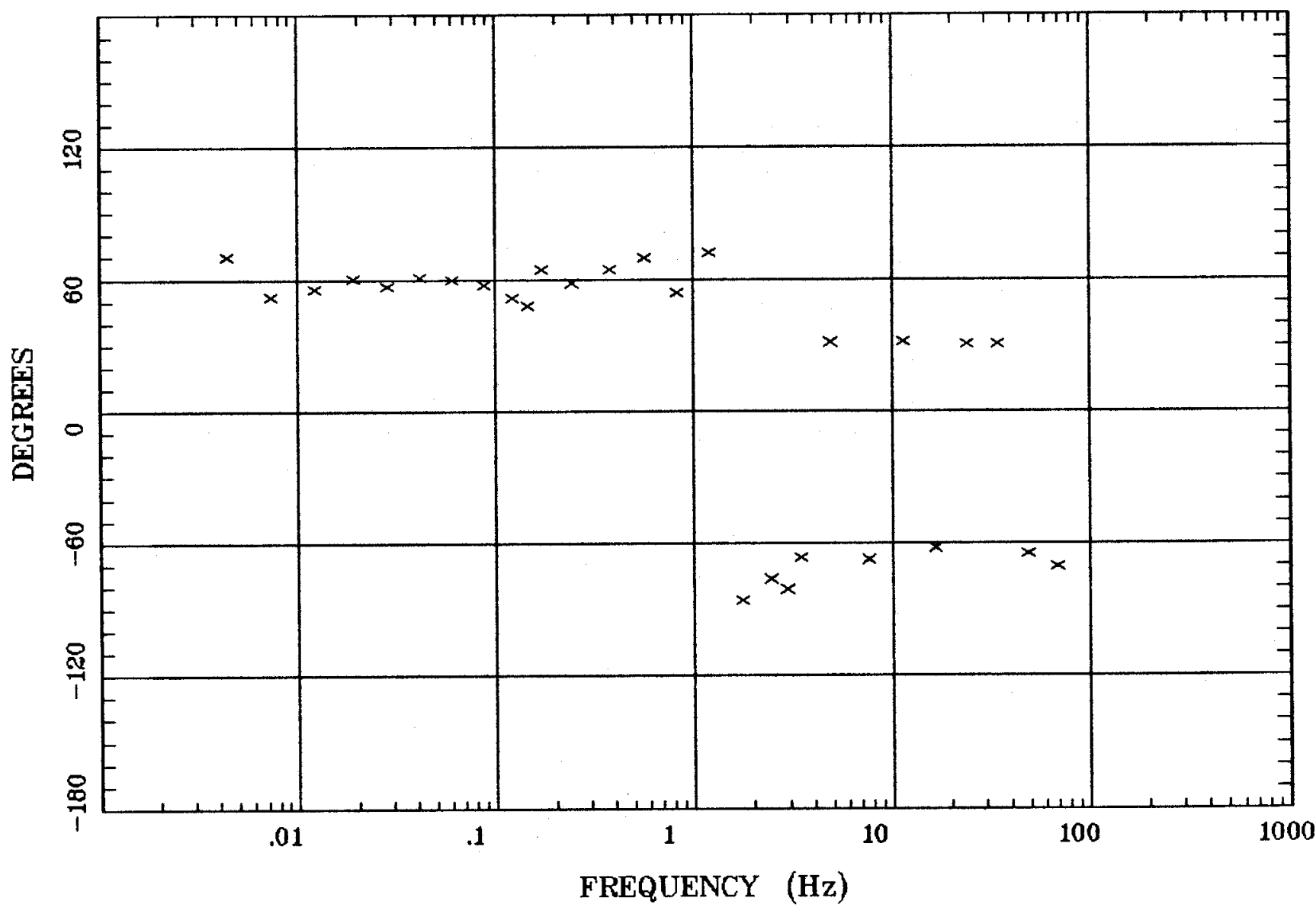

Client:

Remote: Local B

Acquired: 13:4 Aug 04, 1998

Survey Co:USGS GD-MRP Denver
Rotation:

Filename: hr42.avg

Channels: Ch1 Ch2 Ch3 ch4 Ch5 Ch8 Ch9

Platted: 13:50 Dec 07, 2000

$<$ EMI - ElectroMagnetic Instruments > 


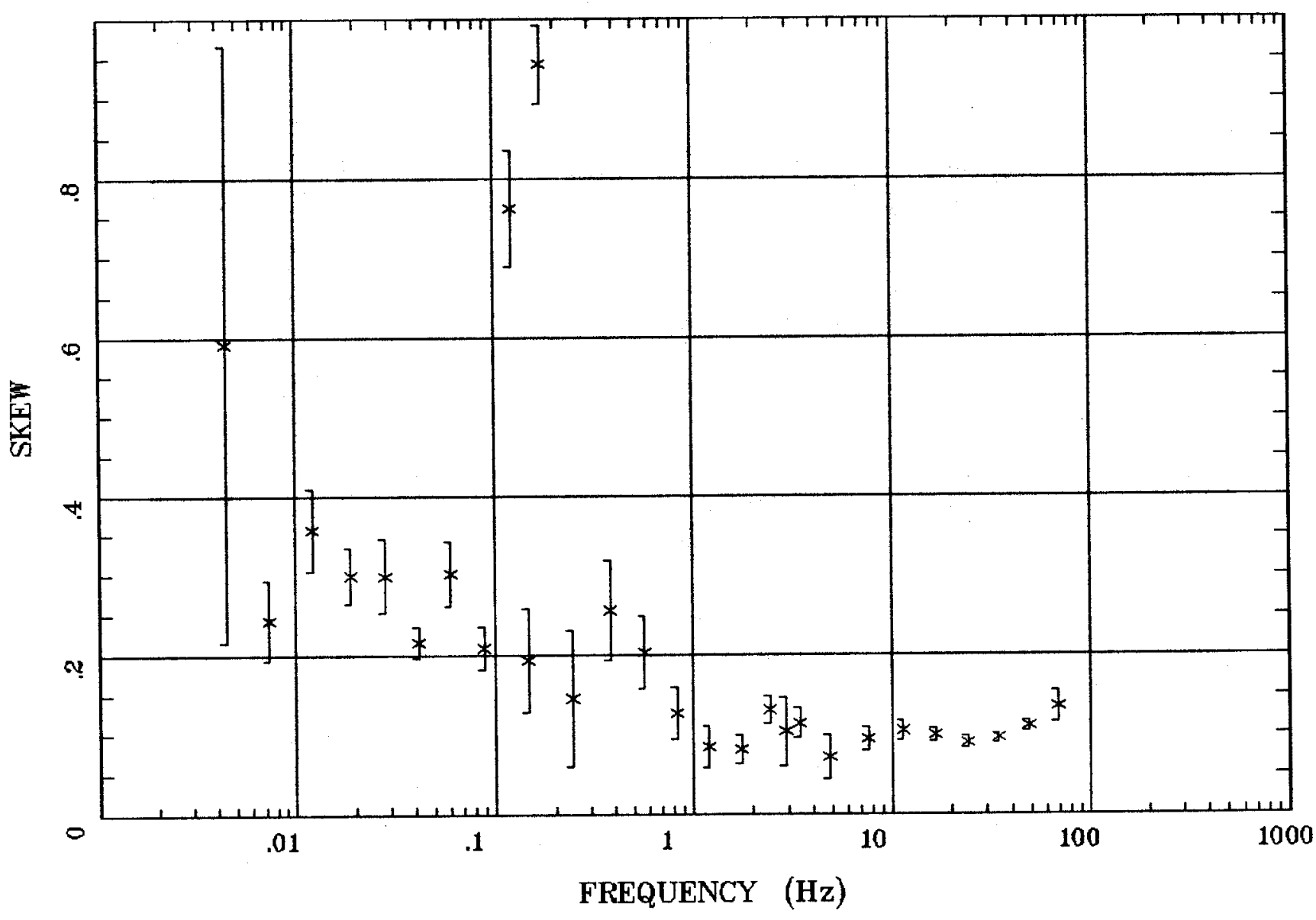

Client:

Remote: Local B

Acquired: 13:4 Aug 04, 1998

Survey Co:USGS GD-MRP Denver

\section{Rotation:}

Filename: hr42.avg

Channels: Ch1 Ch2 Ch3 ch4 ch5 Ch8 Ch9

Plotted: 13:50 Dec 07, 2000

< EMI - ElectroMagnetic Instruments > 
E MULT Coh.

Humboldt River Line 1

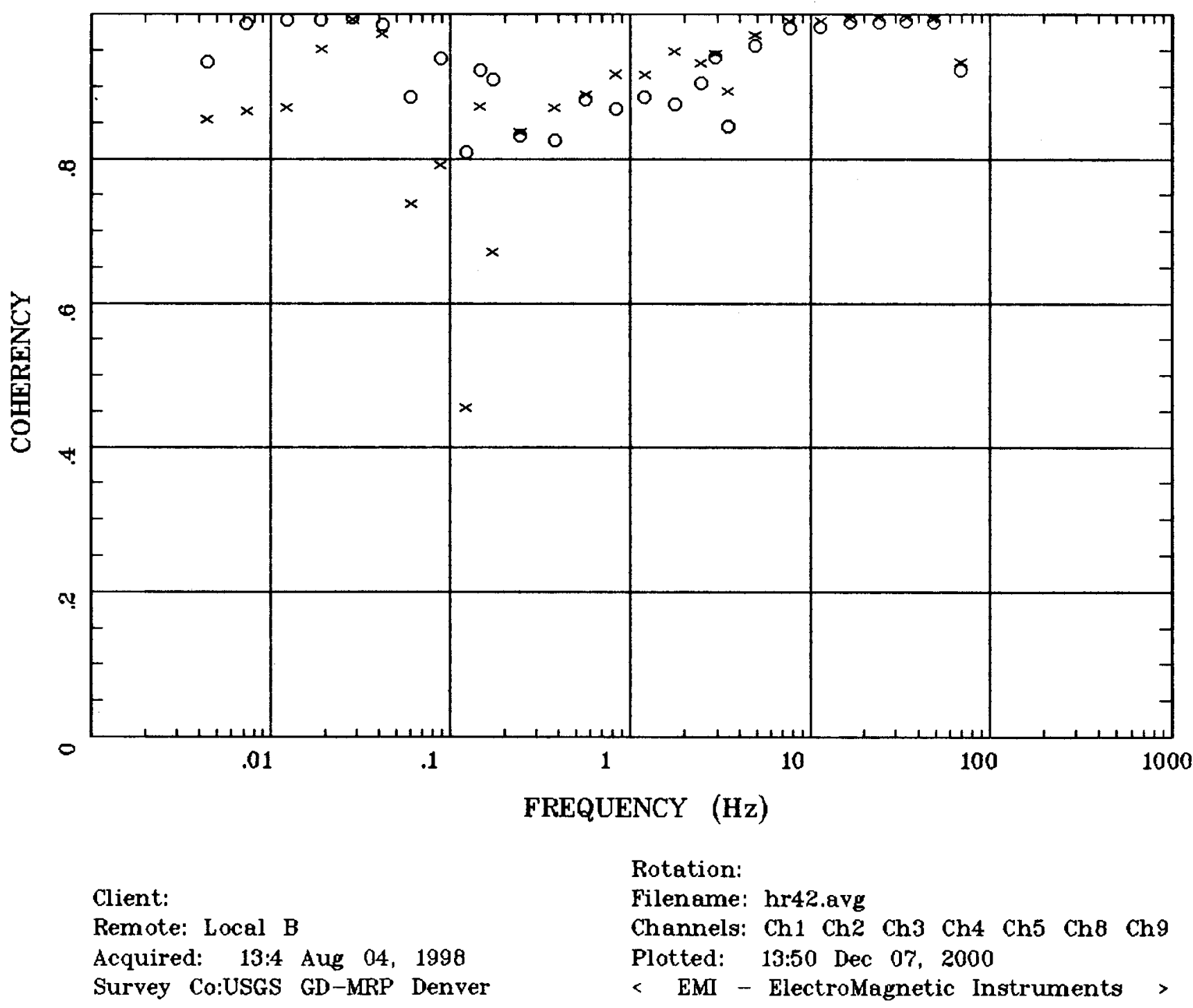


Humboldt River Line 1

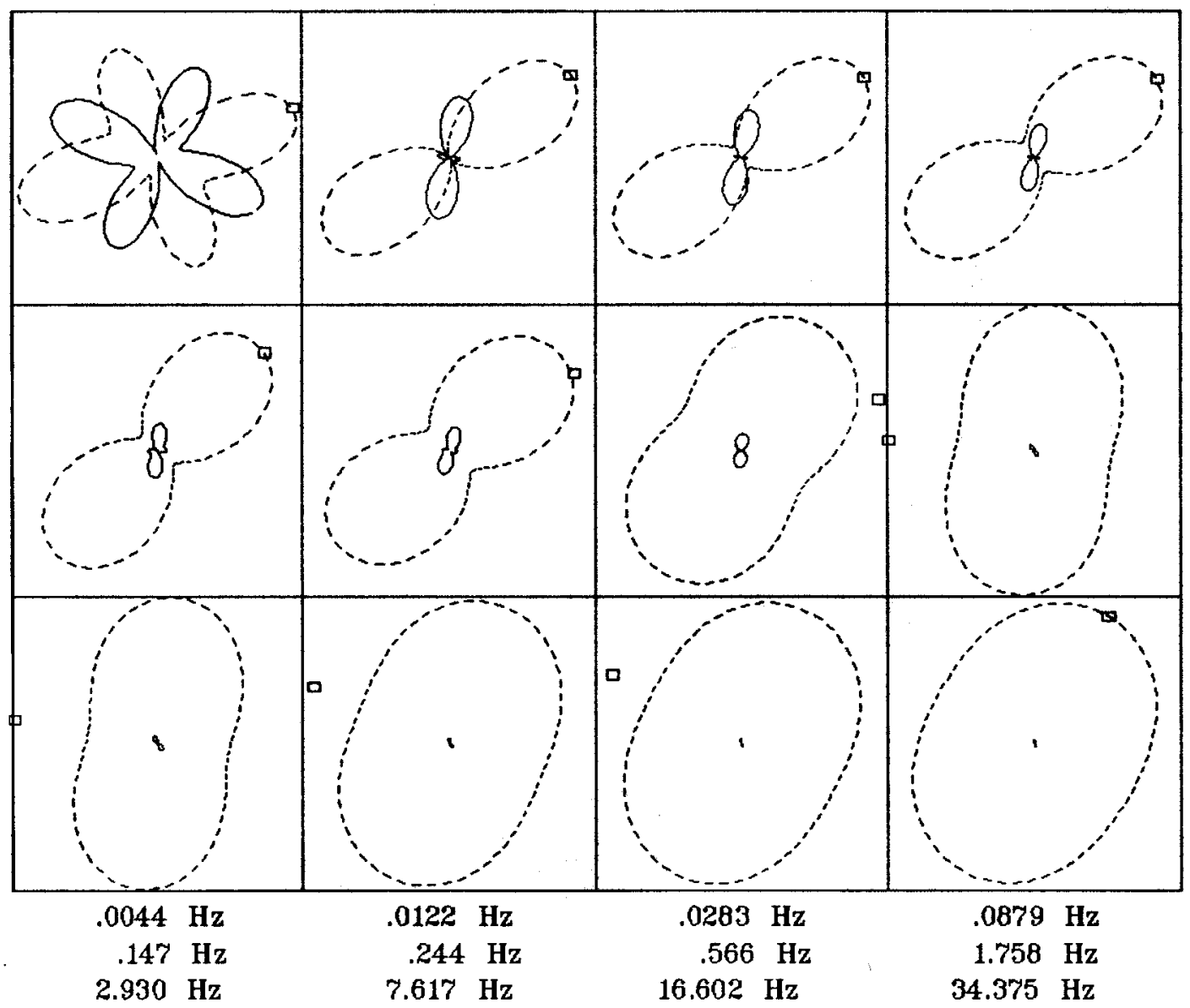

Client:

Remote: Local B

Acquired: 13:4 Aug 04, 1998

Survey Co:USGS GD-MRP Denver
Rotation:

Filename: hr42.avg

Channels: Ch1 Ch2 Ch3 Ch4 Ch5 Ch8 Ch9

Plotted: 13:50 Dec 07, 2000

< EMI - ElectroMagnetic Instruments > 
APPARENT RESISTIVITY

Humboldt River Line 1



Client:

Remote: Local B

Acquired: 09:4 Aug 04, 1998

Survey Co:USGS GD-MRP Denver
Rotation:

Filename: hr59.avg

Channels: Ch1 Ch2 Ch3 Ch4 Ch5 Ch8 Ch9

Plotted: 13:59 Dec 07, 2000

< EMI - ElectroMagnetic Instruments > 
IMPEDANCE PHASE

Humboldt River Line 1

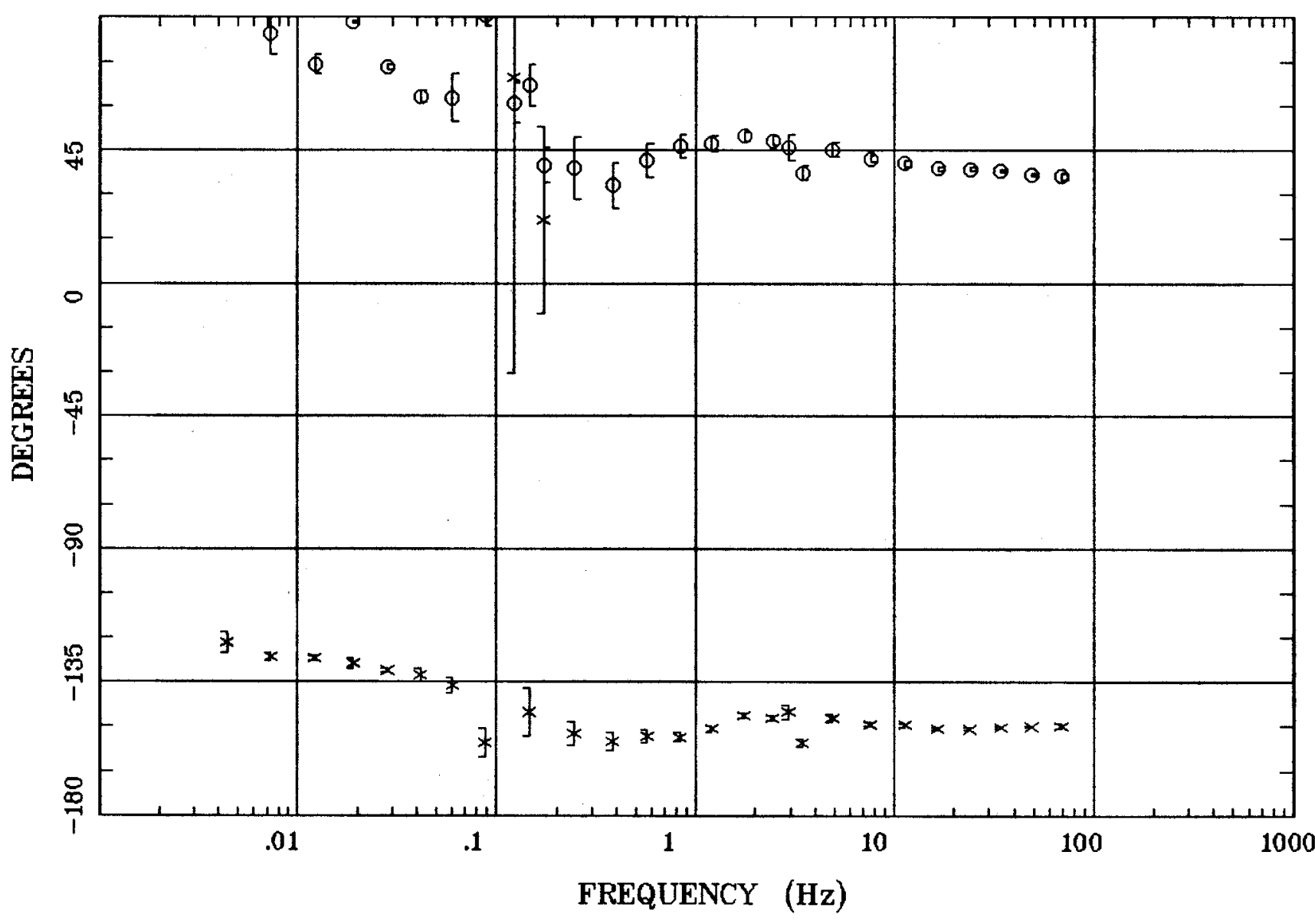

Client:

Remote: Local B

Acquired: 09:4 Aug 04, 1998

Survey Co:USGS GD-MRP Denver
Rotation:

Filename: hr59.avg

Channels: Ch1 Ch2 Ch3 Ch4 Ch5 Ch8 Ch9

Plotted: 13:59 Dec 07, 2000

< EMI - ElectroMagnetic Instruments > 


\section{ROTATION ANGLE}

Humboldt River Line 1

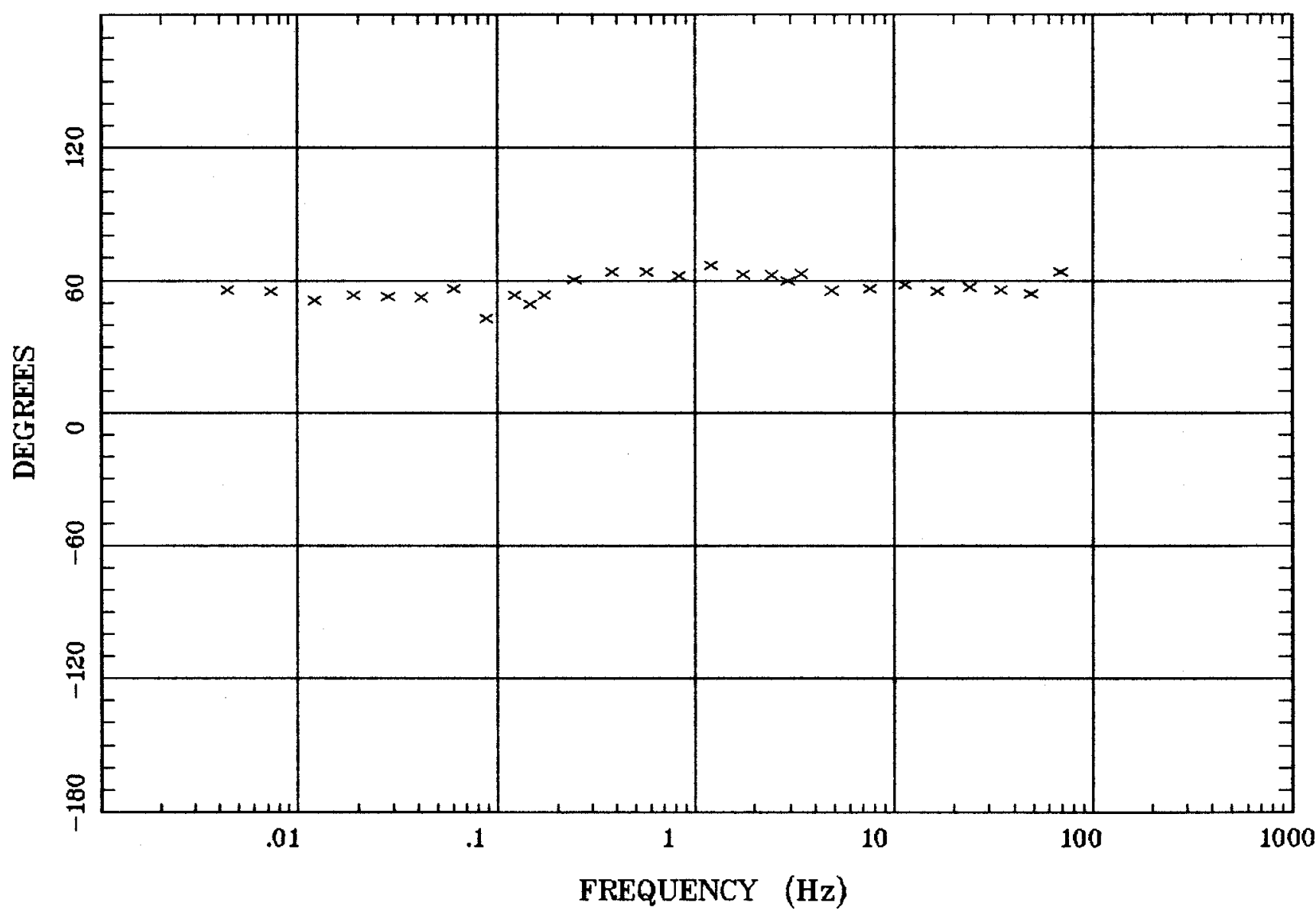

Client:

Remote: Local B

Acquired: 09:4 Aug 04, 1998 Survey Co:USGS GD-MRP Denver
Rotation:

Filename: hr59.avg

Channels: Ch1 Ch2 Ch3 Ch4 Ch5 Ch8 Ch9

Plotted: 13:59 Dec 07, 2000

< EMI - ElectroMagnetic Instruments 
IMPEDANCE SKEW

Humboldt River Line 1



Client:

Remote: Lacal B

Acquired: 09:4 Aug 04, 1998 Survey Co:USGS GD-MRP Denver
Rotation:

Filename: hr59.avg

Channels: Ch1 Ch2 Ch3 Ch4 Ch5 ch8 Ch9

Plotted: 13:59 Dec 07, 2000

$<$ EMI - ElectroMagnetic Instruments 
E MULT Coh.

Humboldt River Line 1

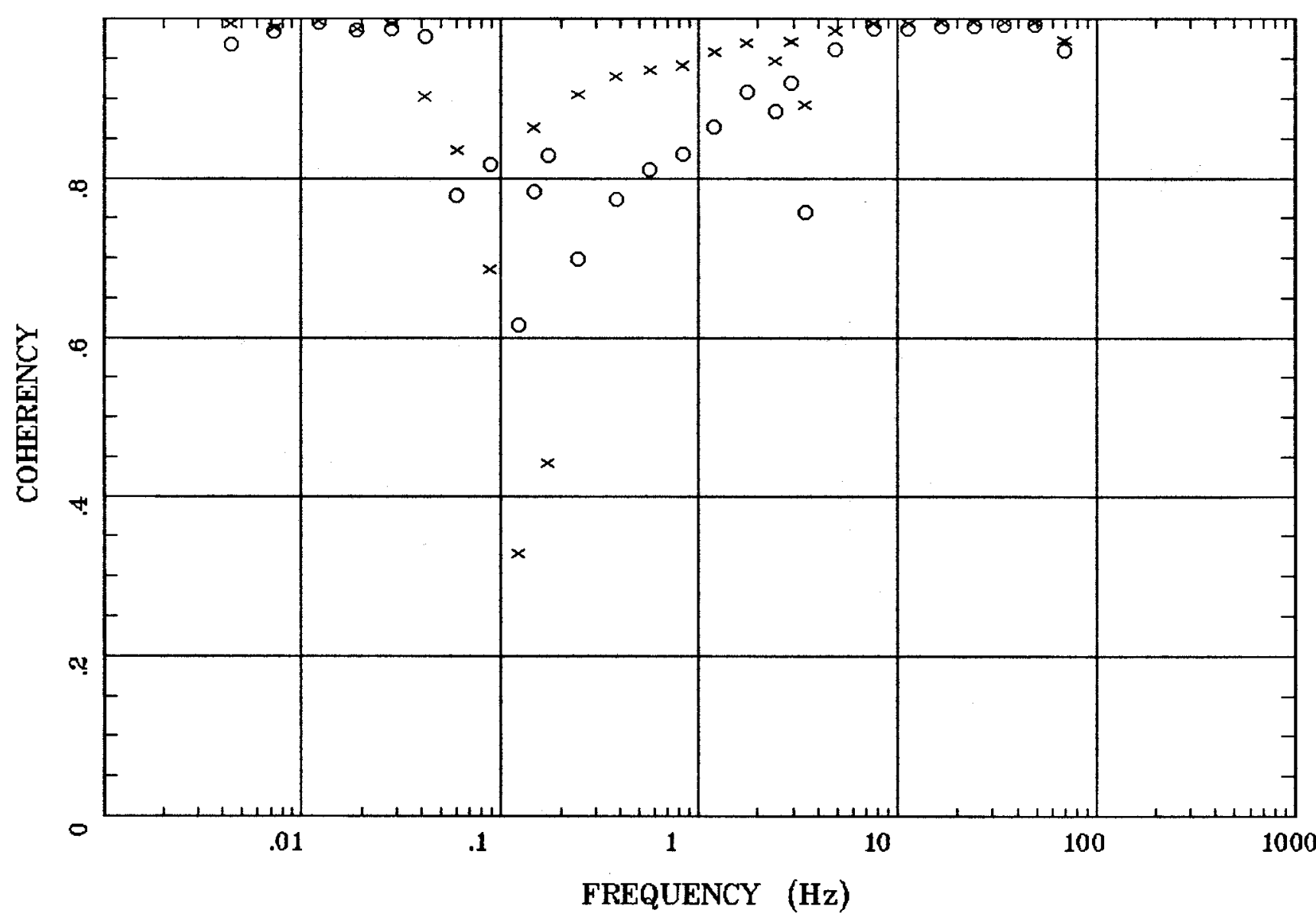

Client:

Remote: Local B

Acquired: 09:4 Aug 04, 1998

Survey Co:USGS GD-MRP Denver
Rotation:

Filename: hr59.avg

Channels: Ch1 Ch2 Ch3 Ch4 Ch5 Ch8 Ch9

Plotted: 13:59 Dec 07, 2000

< EMI - ElectroMagnetic Instruments 


\section{Station 59}

Humboldt River Line 1

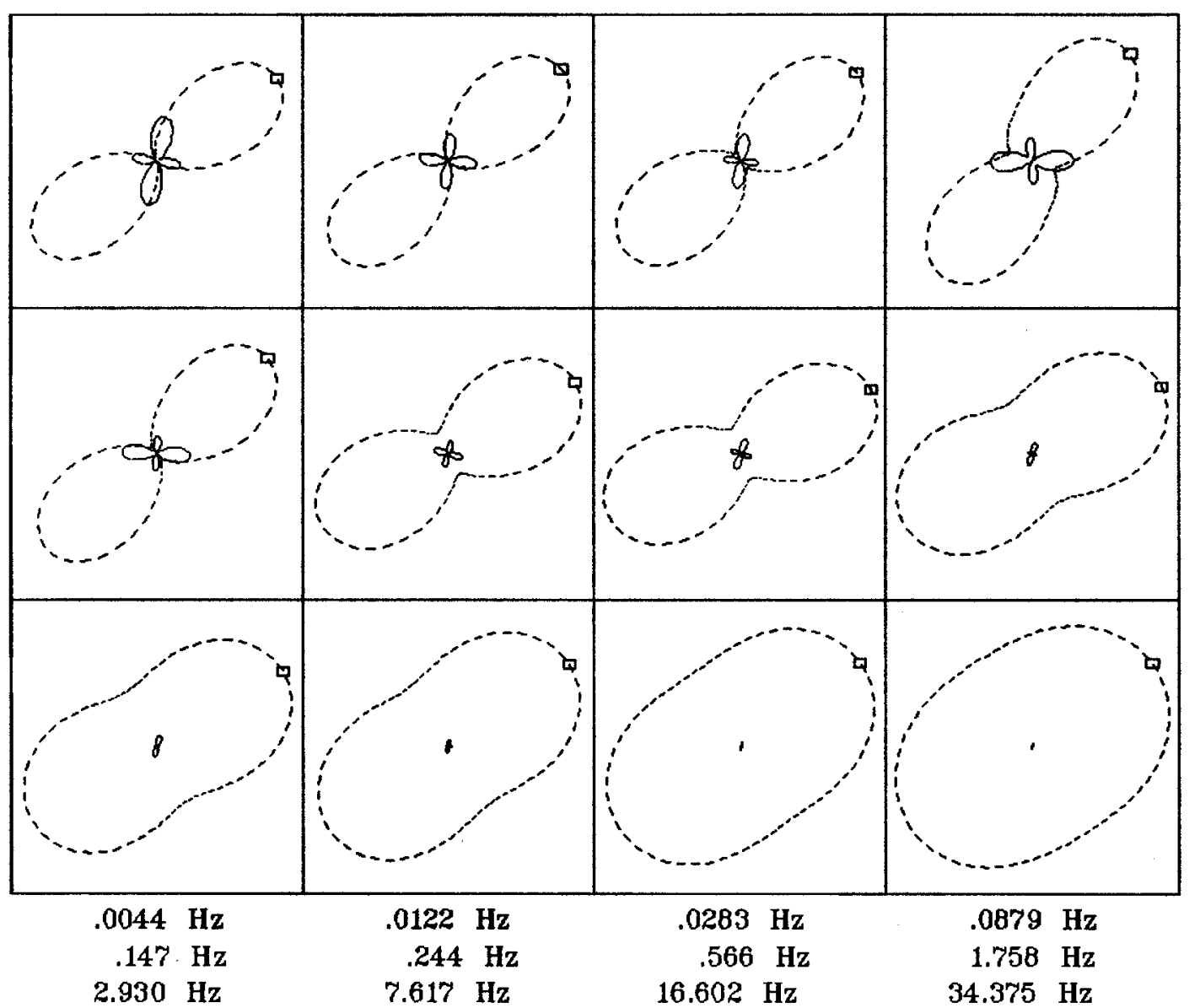

Client:

Remote: Local B

Acquired: 09:4 Aug 04, 1998

Survey Co:USGS GD-MRP Denver
Rotation:

Filename: hr59.avg

Channels: Ch1 Ch2 Ch3 Ch4 Ch5 Ch8 Ch9

Plotted: 13:59 Dec 07, 2000

< EMI - ElectroMagnetic Instruments > 
TIPPER MAGNITUDE

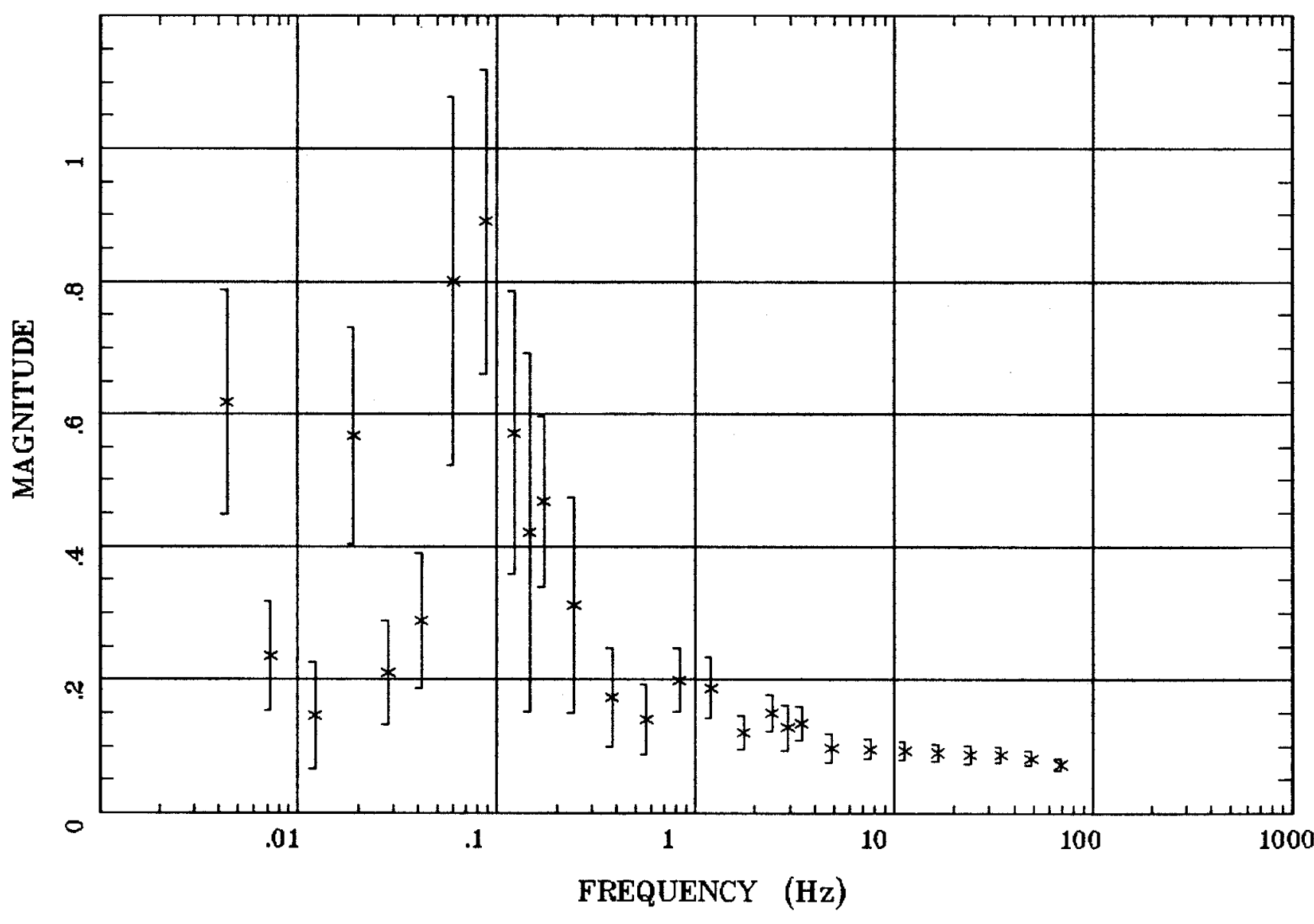

Client:

Remote: Local B

Acquired: 09:4 Aug 04, 1998

Survey Co:USGS GD-MRP Denver
Humboldt River Line 1

Rotation:

Filename: hr59.avg

Channels: Ch1 Ch2 Ch3 Ch4 Ch5 Ch8 Ch9

Plotted: 13:59 Dec 07, 2000

$<$ EMI - ElectroMagnetic Instruments 
Station 59

TIPPER STRIKE

Humboldt River Line 1

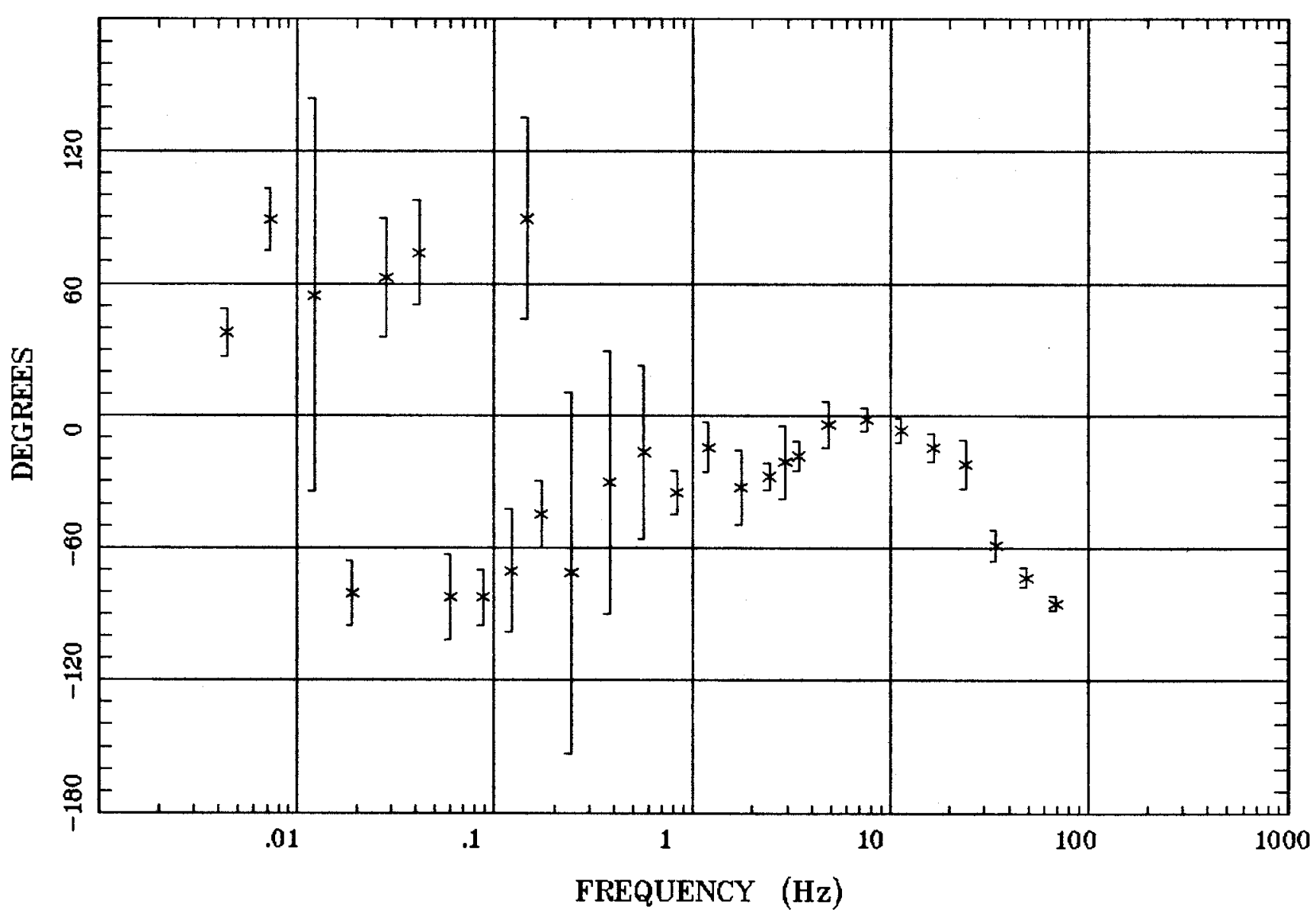

Client:

Remote: Local B

Acquired: 09:4 Aug 04, 1998

ation:

Filename: hr59.avg

Channels: Ch1 Ch2 Ch3 Ch4 Ch5 Ch8 Ch9

Survey Co:USGS GD-MRP Denver

Plotted: 13:59 Dec 07, 2000

$<$ EMI - ElectroMagnetic Instruments 


\section{Station 59}

HzHx.x Coh HzHy.o

Humboldt River Line 1

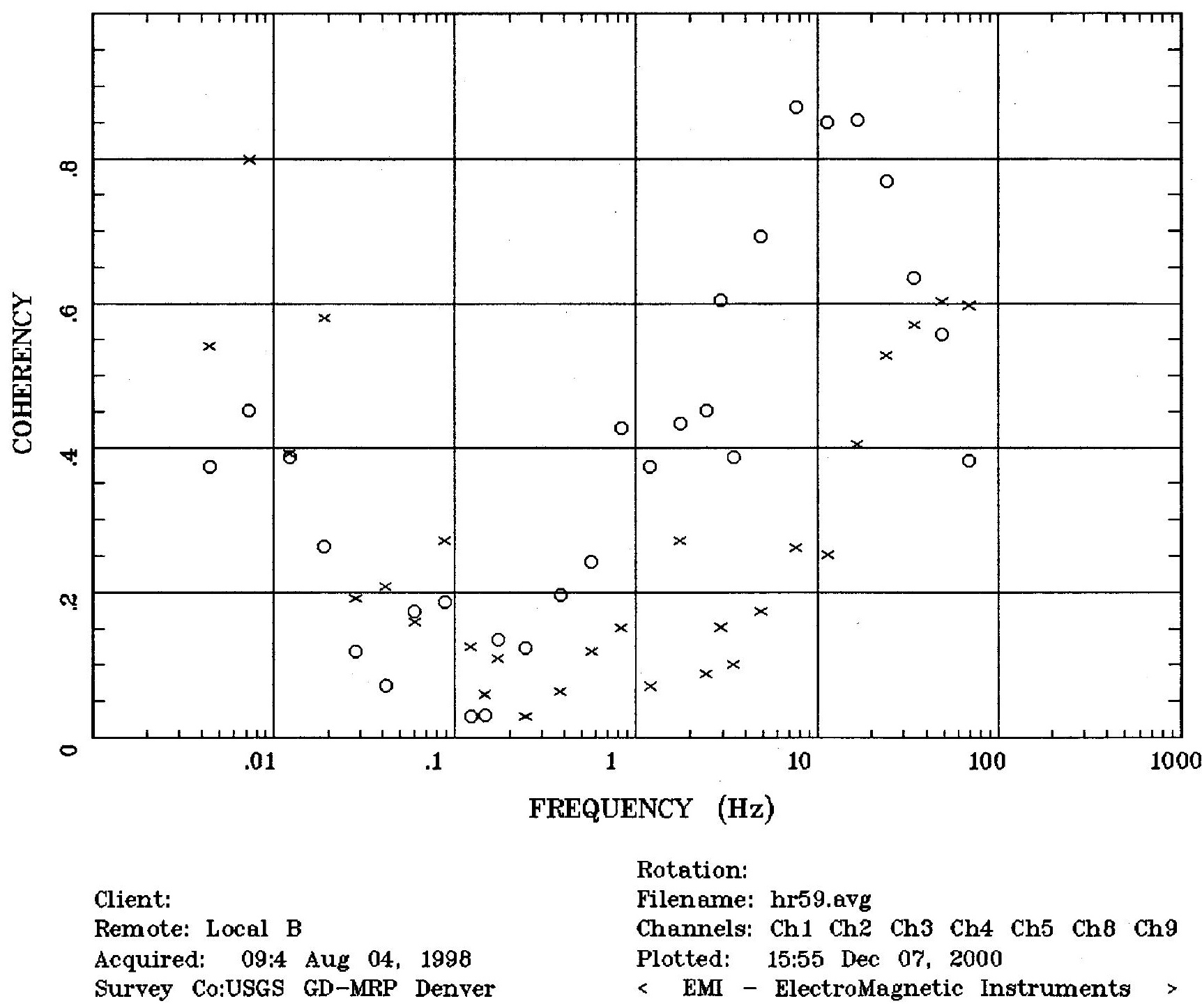


APPARENT RESISTIVITY

Humboldt River Line 1

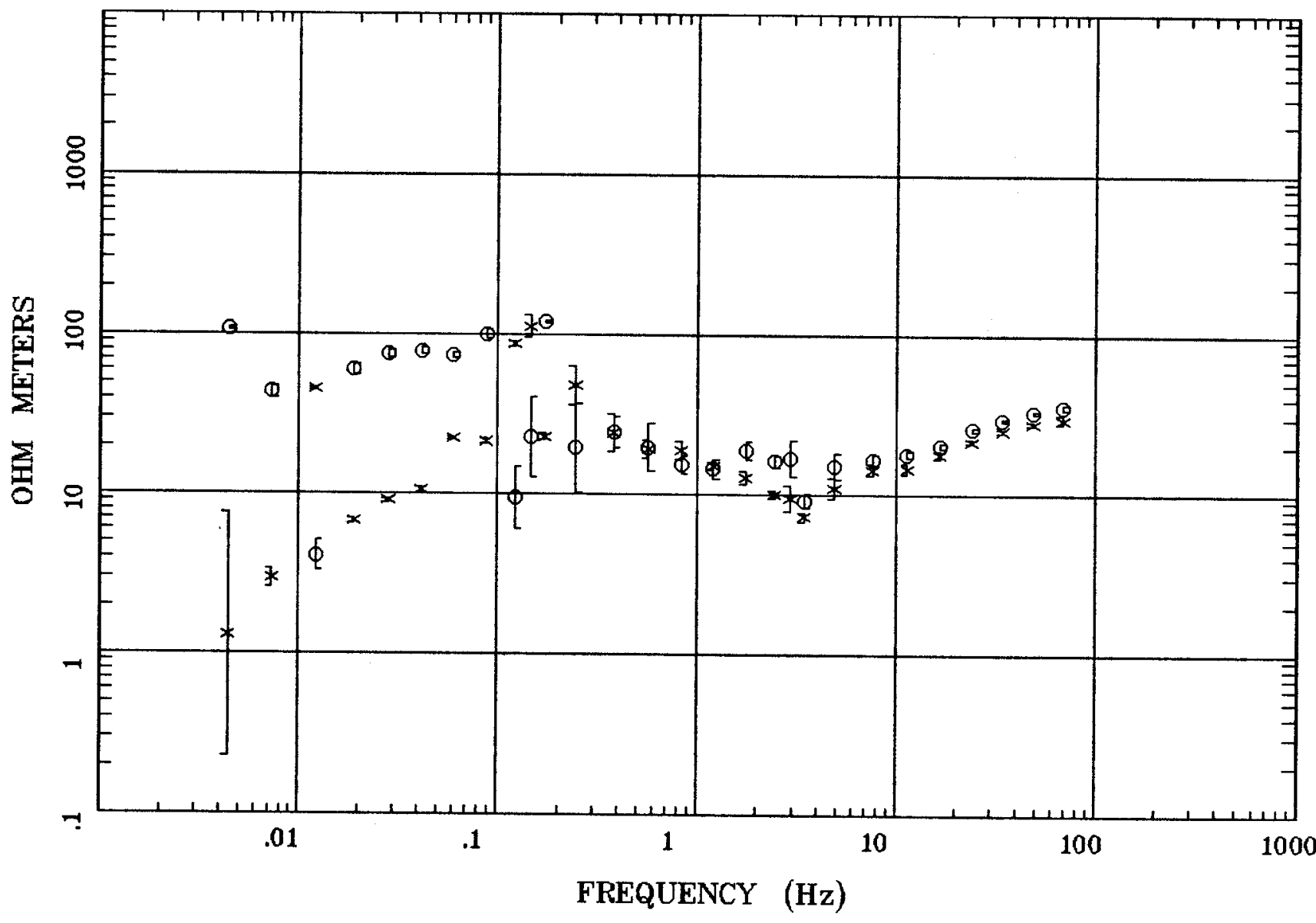

Client:

Remote: Local B

Acquired: 16:2 Aug 03, 1998

Survey Co:USGS GD-MRP Denver
Rotation:

Filename: hr58.avg

Channels: Ch1 Ch2 Ch3 Ch4 Ch5 Ch8 Ch9

Plotted: 13:58 Dec 07, 2000

< EMI - ElectroMagnetic Instruments > 
IMPEDANCE PHASE

Humboldt River Line 1

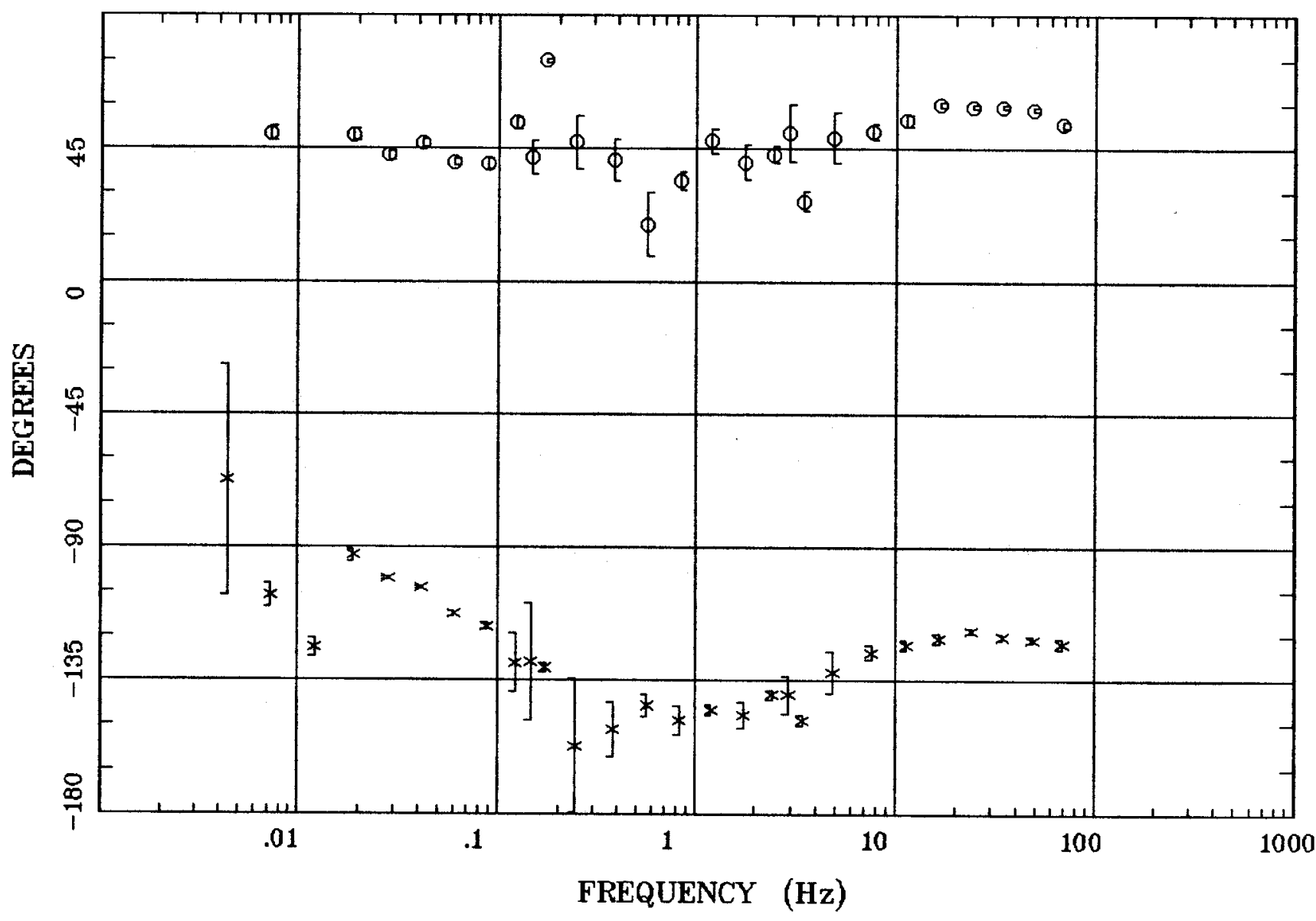

Client:

Remote: Local $B$

Acquired: 16:2 Aug 03, 1998

Survey Co:USGS GD-MRP Denver
Rotation:

Filename: hr58.avg

Channels: Ch1 Ch2 Ch3 Ch4 Ch5 Ch8 Ch9

Platted: 13:58 Dec 07, 2000

< EMI - ElectroMagnetic Instruments 


\section{Station 58}

ROTATION ANGLE

Humboldt River Line 1

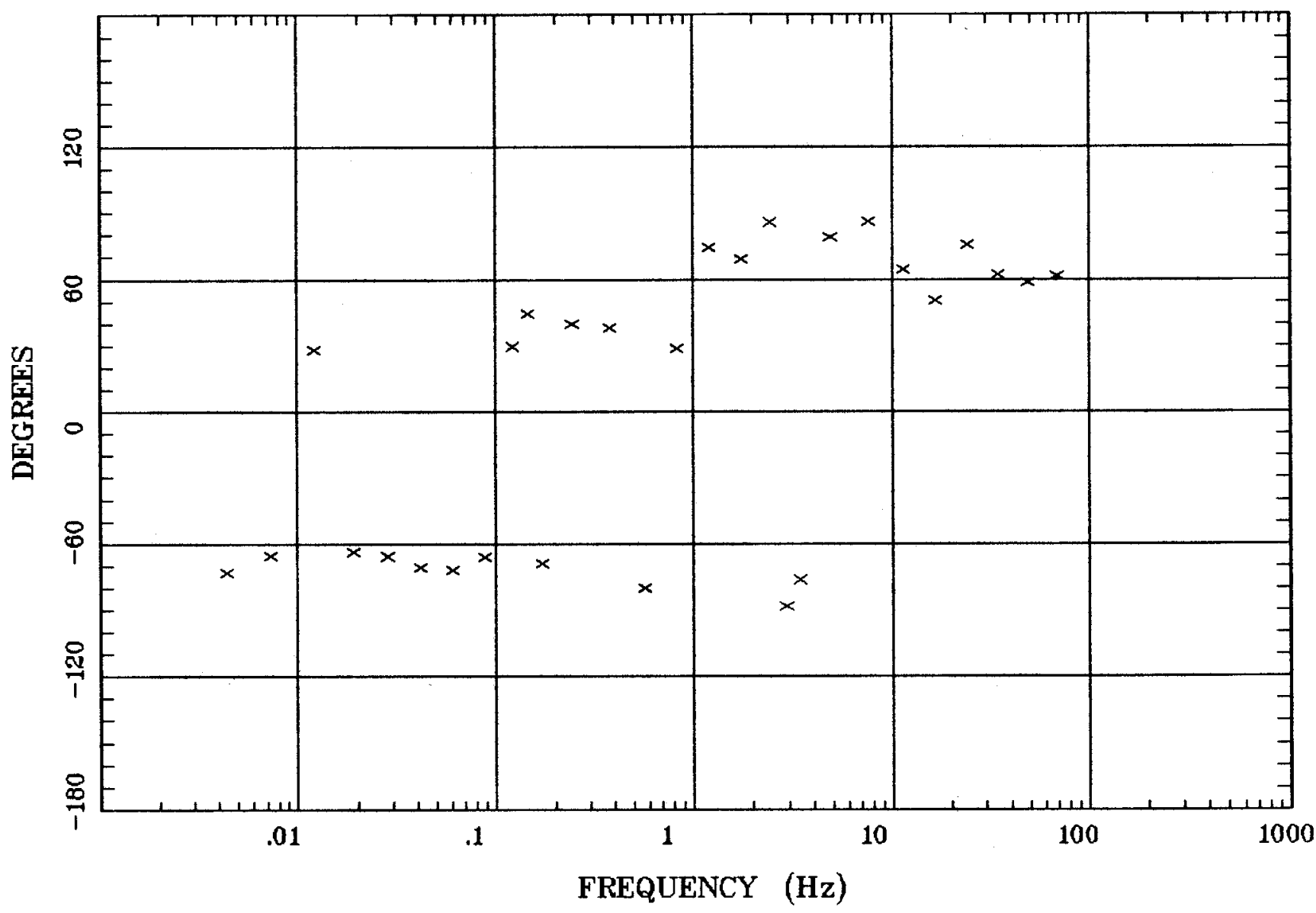

Client:

Remote: Local B

Acquired: 16:2 Aug 03, 1998

Survey Co:USGS GD-MRP Denver

Ratation:

Filename: hr58.avg

Channels: Ch1 Ch2 Ch3 Ch4 Ch5 Ch8 Ch9

Plotted: 13:58 Dec 07, 2000

< EMI - ElectroMagnetic Instruments > 




Client:

Remote: Local B

Acquired: 16:2 Aug 03, 1998 Survey Co:USGS GD-MRP Denver
Rotation:

Filename: hr58.avg

Channels: Ch1 Ch2 Ch3 ch4 Ch5 Ch8 Ch9

Plotted: 13:58 Dec 07, 2000

$<$ EMI - ElectroMagnetic Instruments 


\section{Station 58}

E MULT Coh.

Humboldt River Line 1

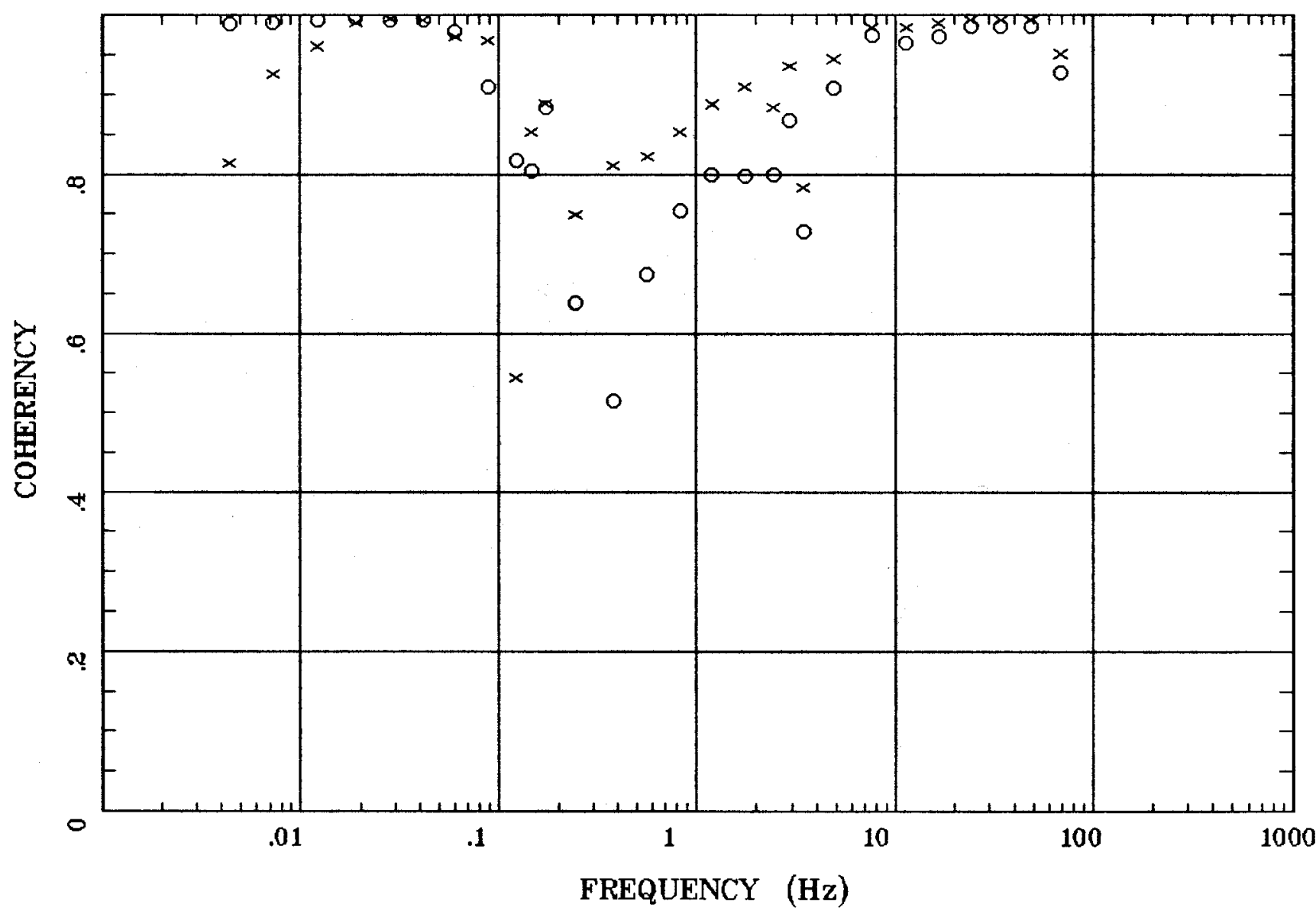

Client:

Remote: Local $B$

Acquired: 16:2 Aug 03, 1998 Survey Co:USGS GD-MRP Denver
Rotation:

Filename: hr58.avg

Channels: Ch1 Ch2 Ch3 Ch4 Ch5 Ch8 Ch9

Plotted: 13:58 Dec 07, 2000

$<$ EMI - ElectroMagnetic Instruments 
Humboldt River Line 1

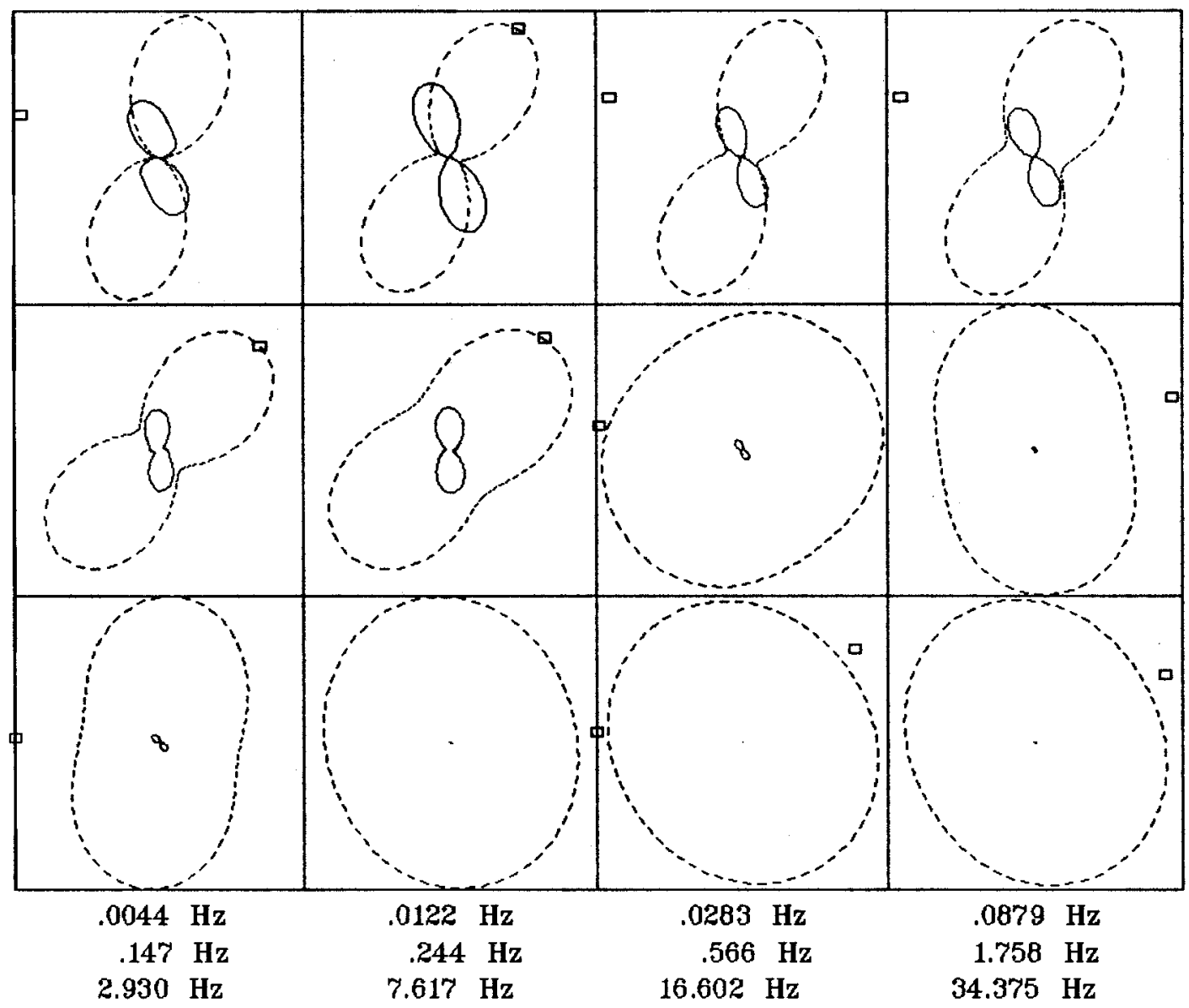

Client:

Remote: Local $B$

Acquired: 16:2 Aug 03, 1998

Survey Co:USGS GD-MRP Denver
Rotation:

Filename: hr58.avg

Channels: Ch1 Ch2 Ch3 Ch4 Ch5 Ch8 Ch9 Plotted: 13:58 Dec 07, 2000

< EMI - ElectroMagnetic Instruments > 
APPARENT RESISTIVITY

Humboldt River Line 1

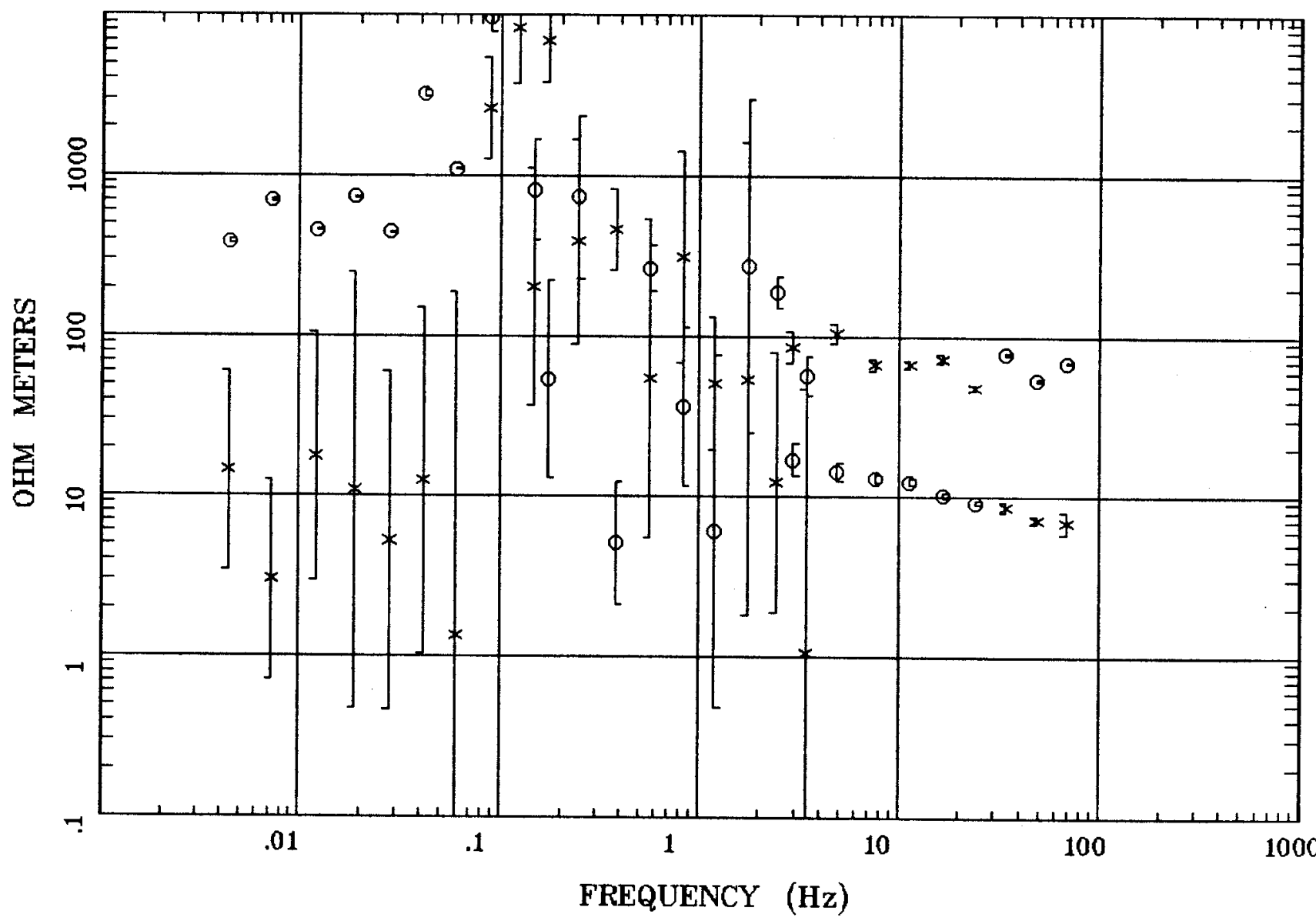

Client:

Remote: Local E

Acquired: 11:1 Aug 03, 1998

Survey Co:USGS GD-MRP Denver
Rotation:

Filename: hr57.avg

Channels: Ch1 Ch2 Ch3 Ch4 Ch5 Ch6 Ch7

Plotted: 13:57 Dec 07, 2000

< EMI - ElectroMagnetic Instruments 
IMPEDANCE PHASE

Humboldt River Line 1



Client:

Remote: Local E

Acquired: 11:1 Aug 03, 1998

Survey Co:USGS GD-MRP Denver
Rotation:

Filename: hr57.avg

Channels: Ch1 Ch2 Ch3 Ch4 Ch5 Ch6 Ch7

Platted: 13:57 Dec 07, 2000

< EMI - ElectroMagnetic Instruments > 


\section{Station 57}

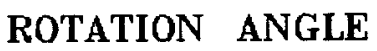

Humboldt River Line 1

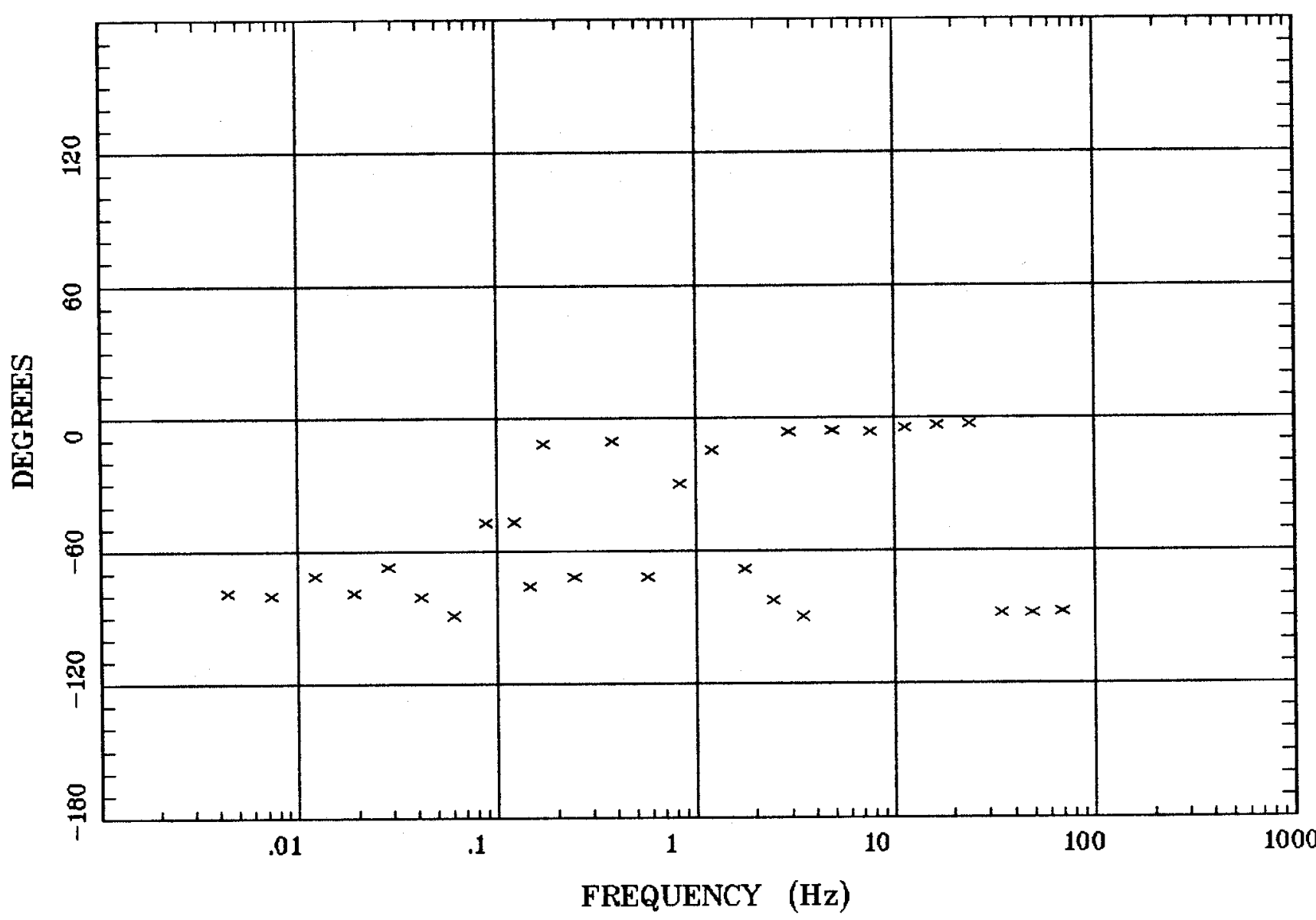

Client:

Remote: Local E

Acquired: 11:1 Aug 03, 1998 Survey Co:USGS GD-MRP Denver
Rotation:

Filename: hr57.avg

Channels: Ch1 Ch2 Ch3 Ch4 Ch5 Ch6 Ch7

Plotted: 13:57 Dec 07, 2000

$<$ EMI - ElectroMagnetic Instruments 


\section{IMPEDANCE SKEW}

\section{Humboldt River Line 1}

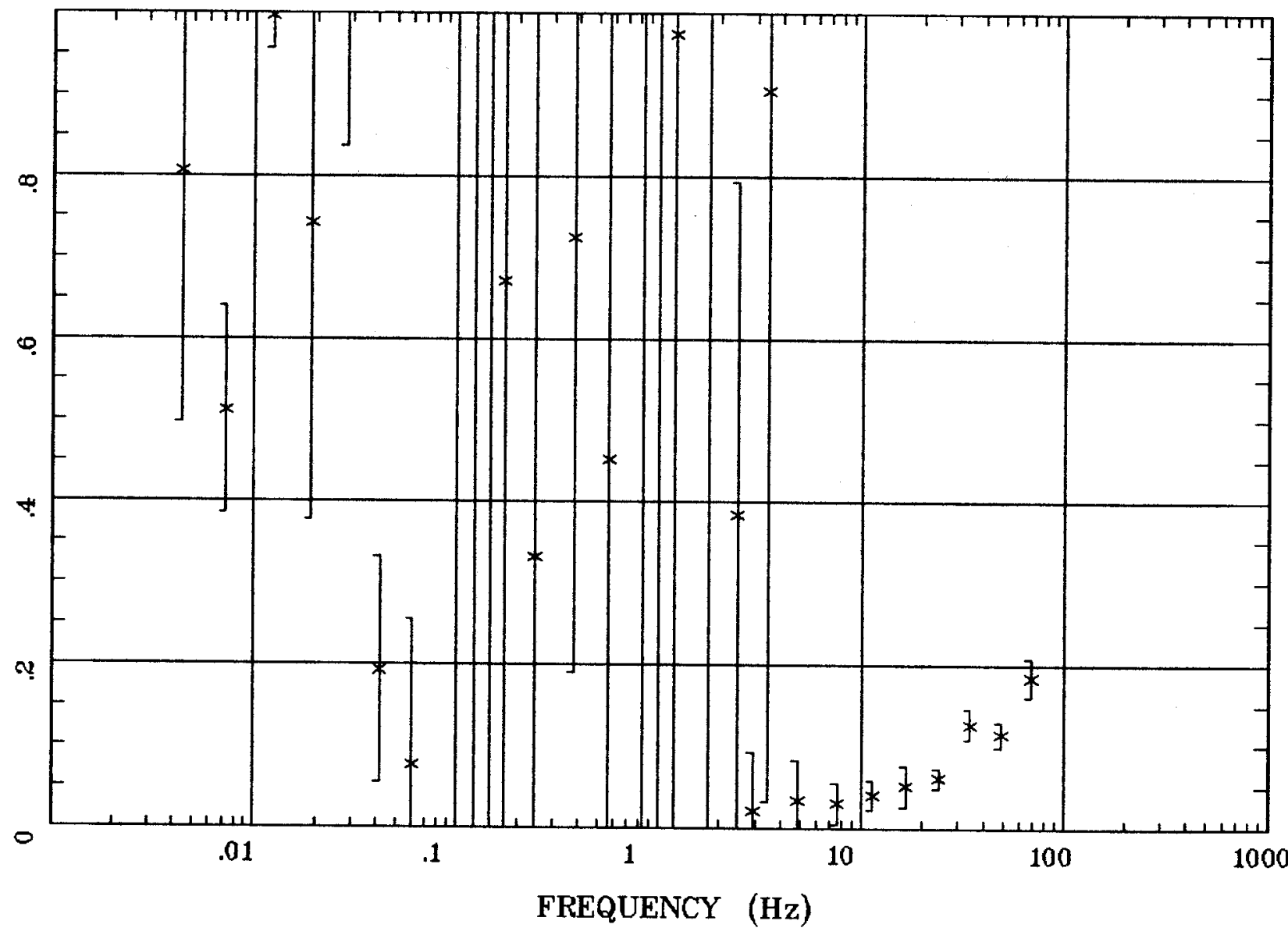

Client:

Remote: Local E

Acquired: 11:1 Aug 03, 1998

Survey Co:USGS GD-MRP Denver
Rotation:

Filename: hr57.avg

Channels: Ch1 Ch2 Ch3 Ch4 Ch5 Ch6 Ch7 Plotted: 13:57 Dec 07, 2000

$\langle$ EMI - ElectroMagnetic Instruments > 
Station 57

E MULT Coh.

Humboldt River Line 1

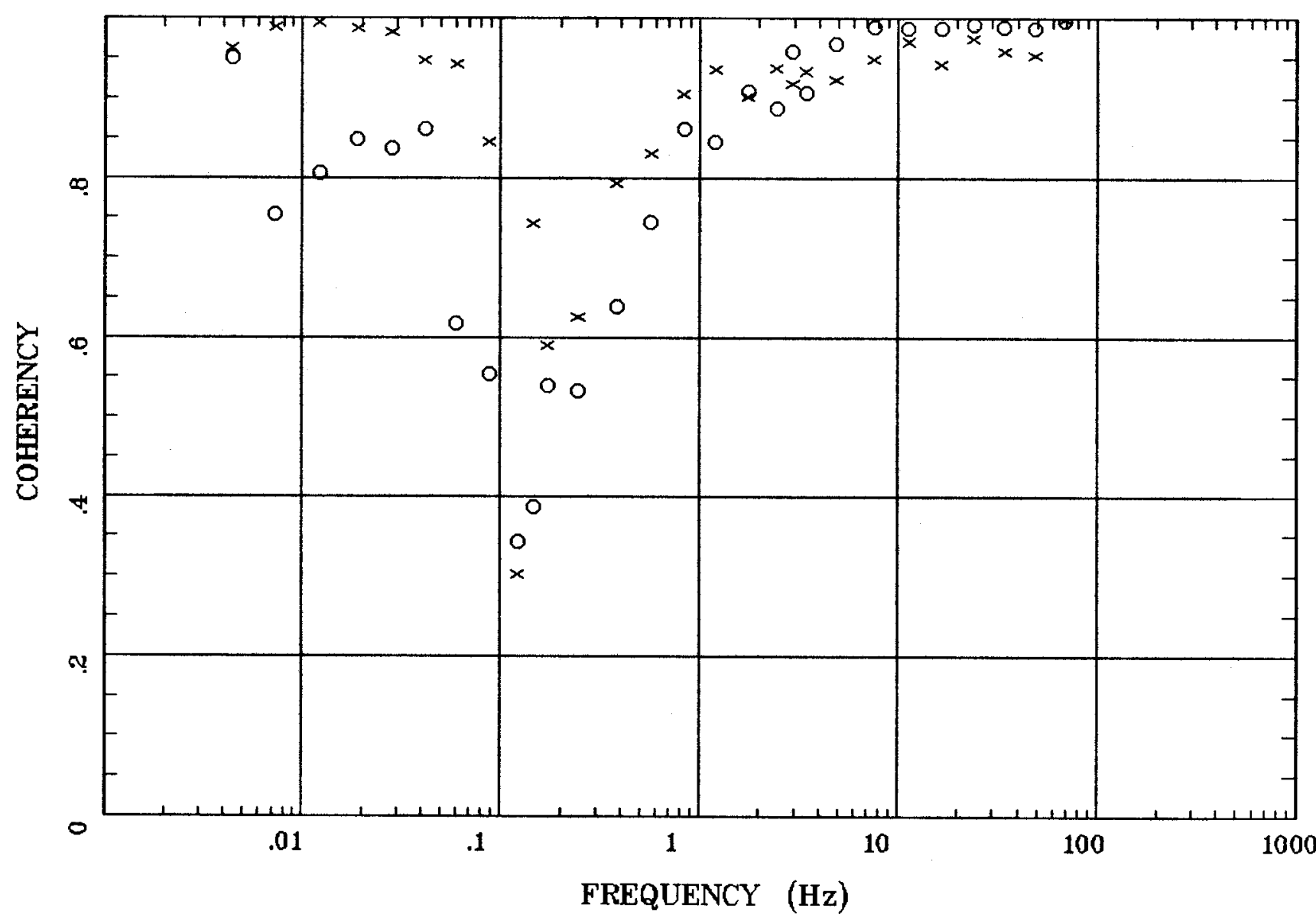

Client:

Remote: Local E

Acquired: 11:1 Aug 03, 1998

Survey Co:USGS GD-MRP Denver
Rotation:

Filename: hr57.avg

Channels: Ch1 Ch2 Ch3 Ch4 Ch5 Ch6 Ch7

Plotted: 13:57 Dec 07, 2000

$<$ EMI - ElectroMagnetic Instruments > 
Humboldt River Line 1

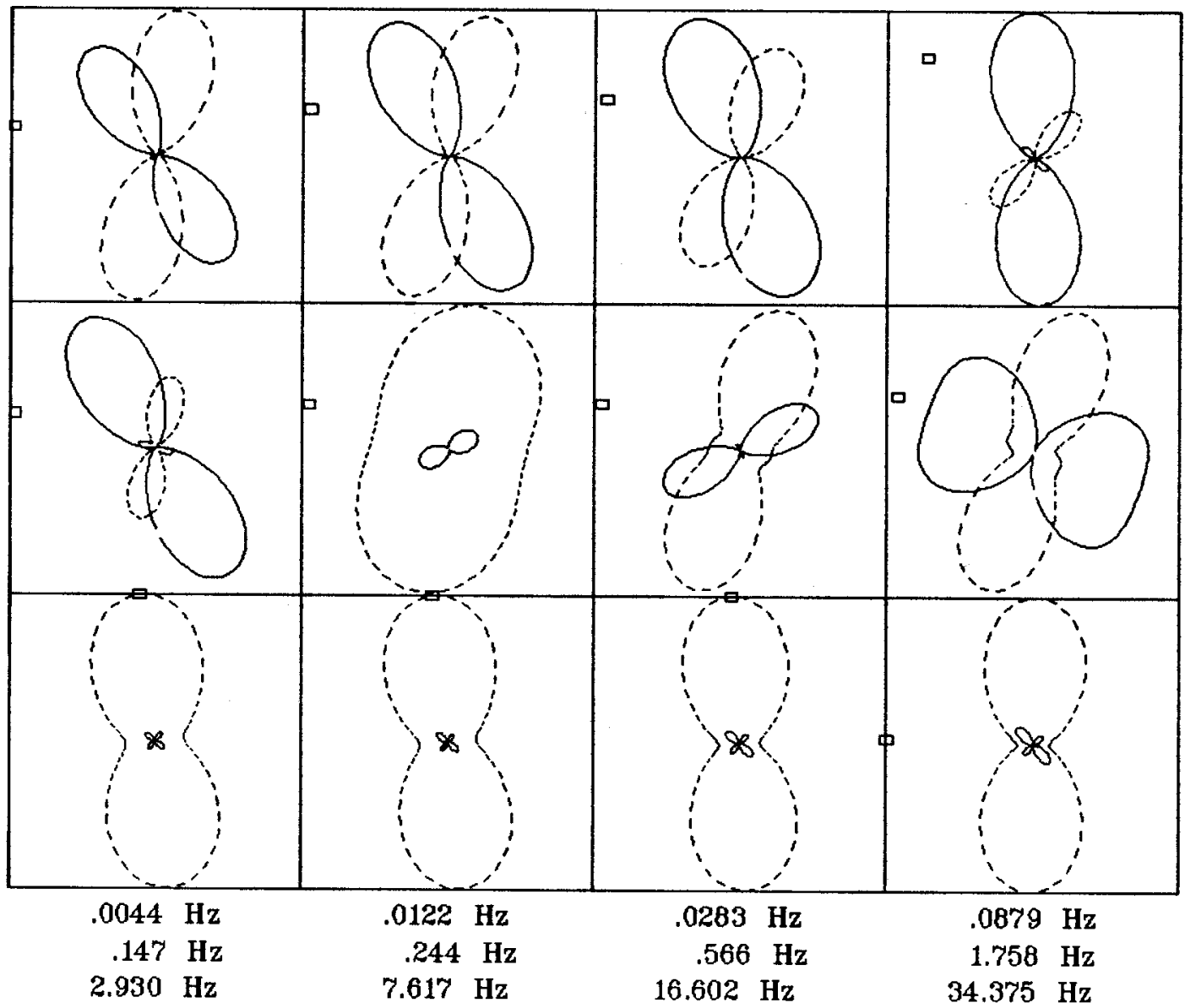

Client:

Remote: Local E

Acquired: 11:1 Aug 03, 1998

Survey Co:USGS GD-MRP Denver
Rotation:

Filename: hr57,avg

Channels: Ch1 Ch2 Ch3 Ch4 Ch5 Ch6 Ch7 Plotted: 13:57 Dec 07, 2000

< EMI - ElectroMagnetic Instruments > 


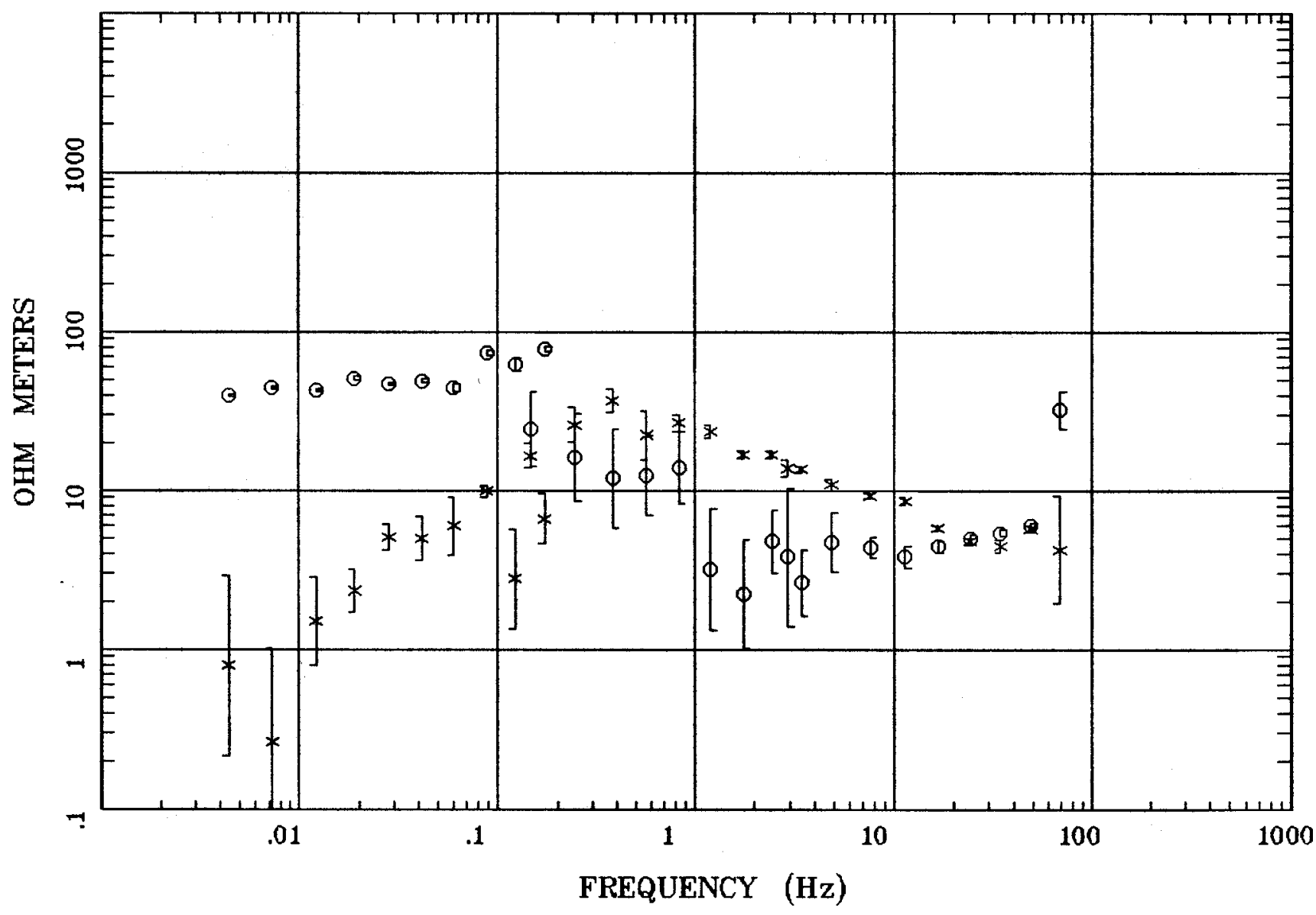

Client:

Remote: Local B

Acquired: 10:1 Aug 01, 1998

Survey Co:USGS GD-MRP Denver
Rotation:

Filename: hr34.avg

Channels: Ch1 Ch2 Ch3 Ch4 Ch5 Ch8 Ch9

Plotted: 13:43 Dec 07, 2000

< EMI - ElectroMagnetic Instruments > 


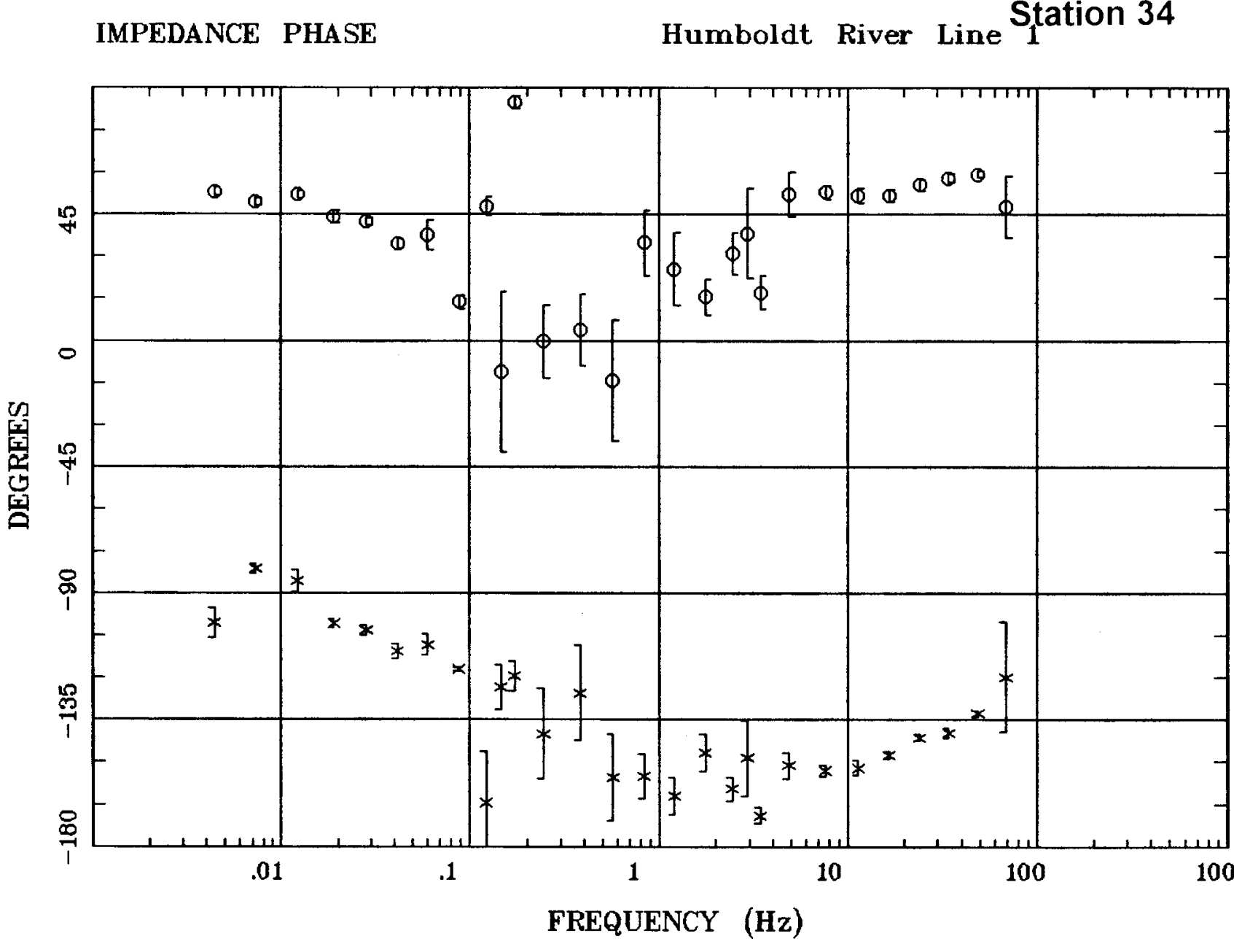

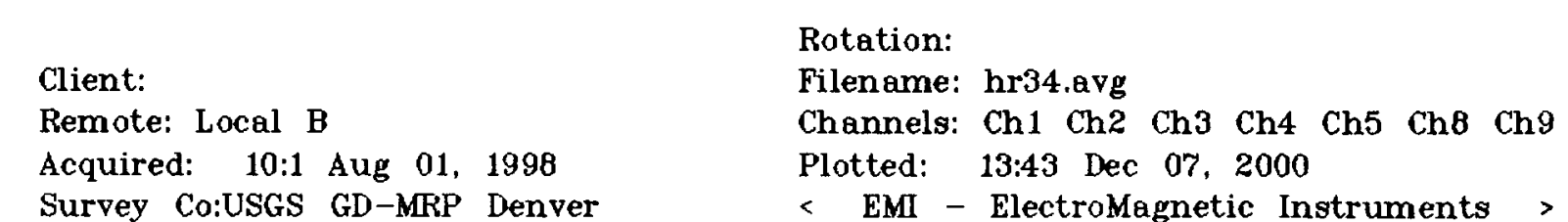




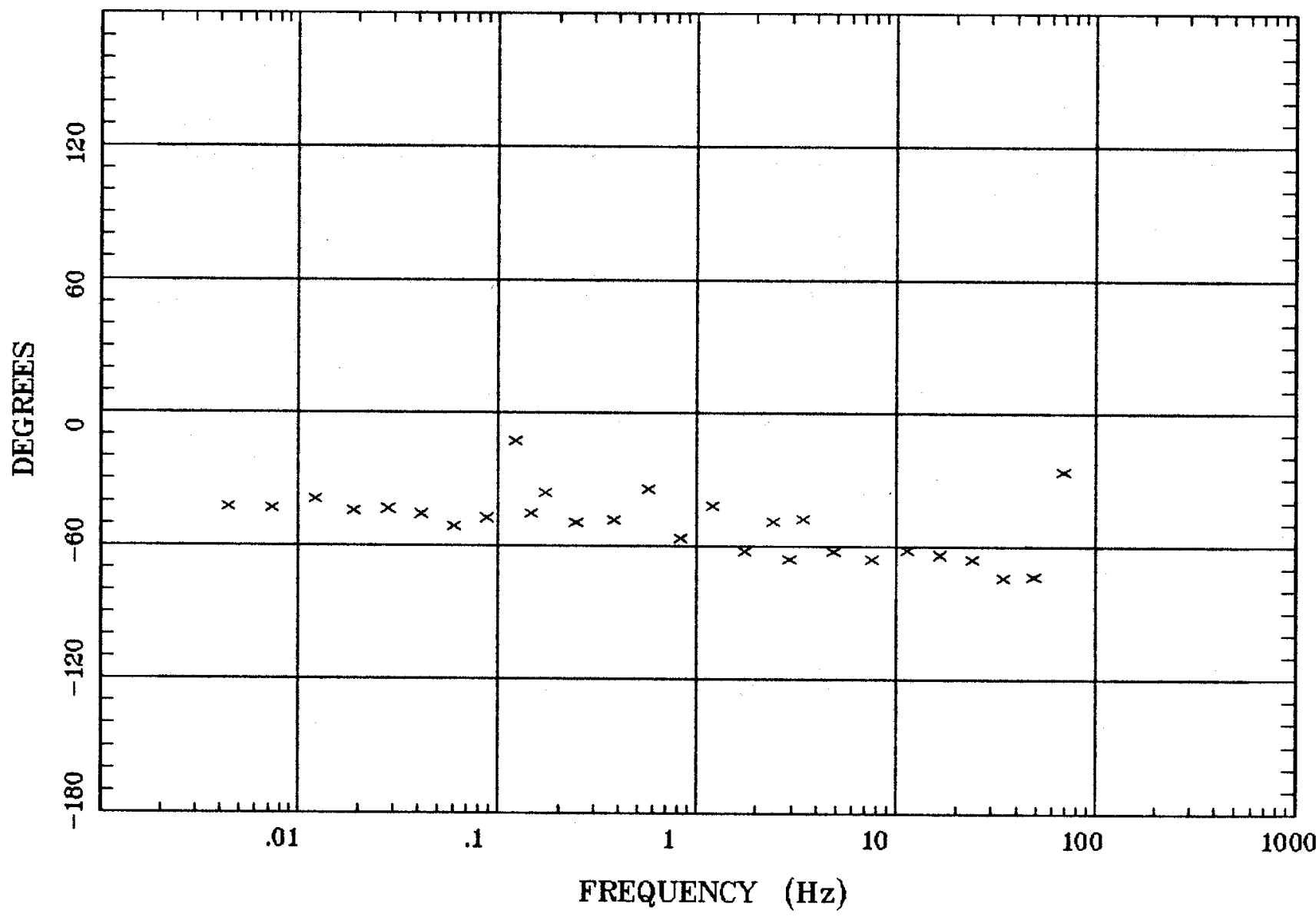

\section{Client:}

Remote: Local B

Acquired: 10:1 Aug 01, 1998 Survey Co:USGS GD-MRP Denver
Rotation:

Filename: hr34.avg

Channels: Ch1 Ch2 Ch3 Ch4 Ch5 Ch8 Ch9 Plotted: 13:43 Dec 07, 2000

$<$ EMI - ElectroMagnetic Instruments 
Humboldt River Line 1

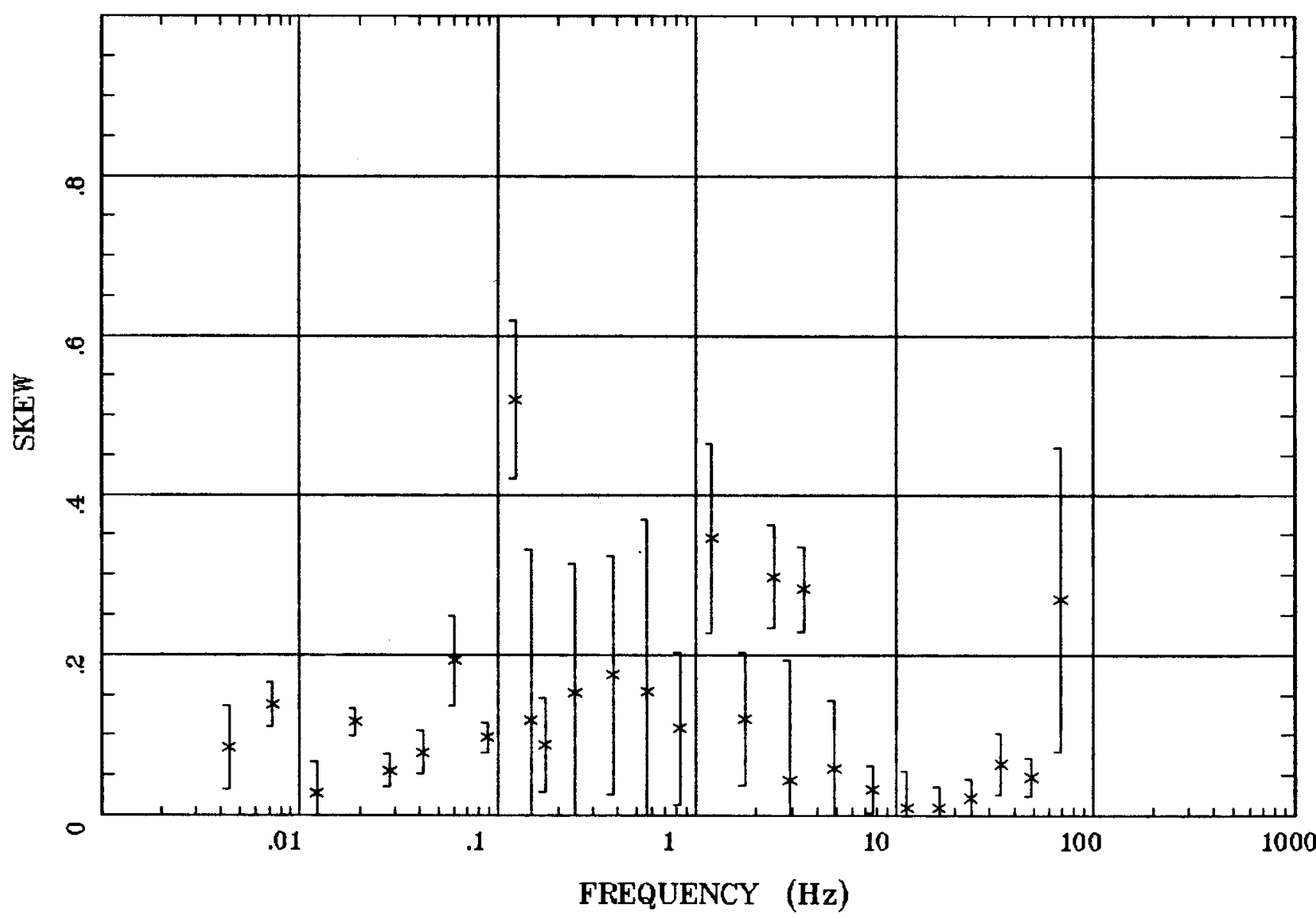

Client:

Remote: Local $B$

Acquired: 10:1 Aug 01, 1998

Survey Co:USGS GD-MRP Denver
Rotation:

Filename: hr34.avg

Channels: Ch1 Ch2 Ch3 Ch4 Ch5 Ch8 Ch9

Plotted: 13:43 Dec 07, 2000

< EMI - ElectroMagnetic Instruments 
E MULT Coh.

Humboldt River Line 1

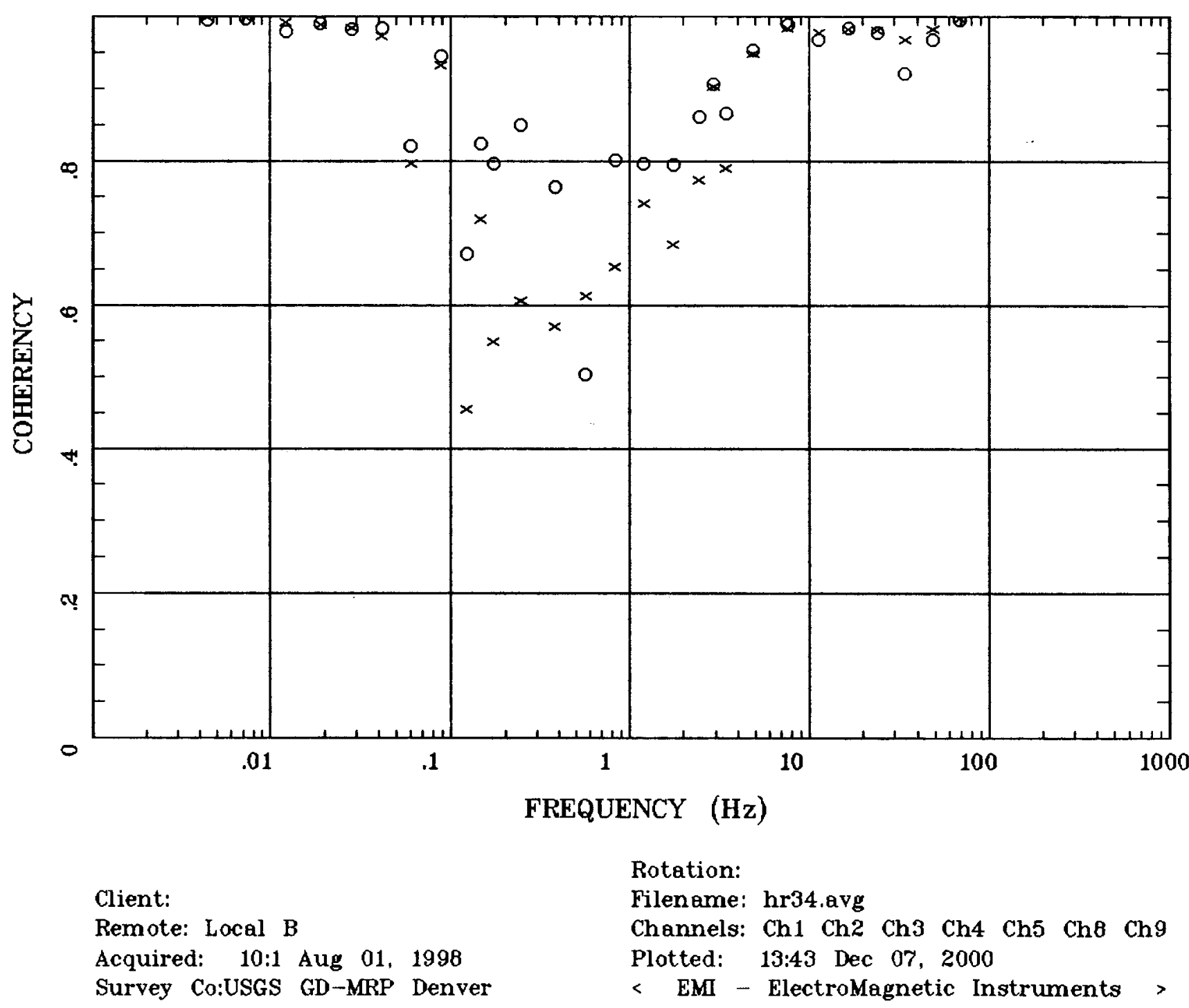




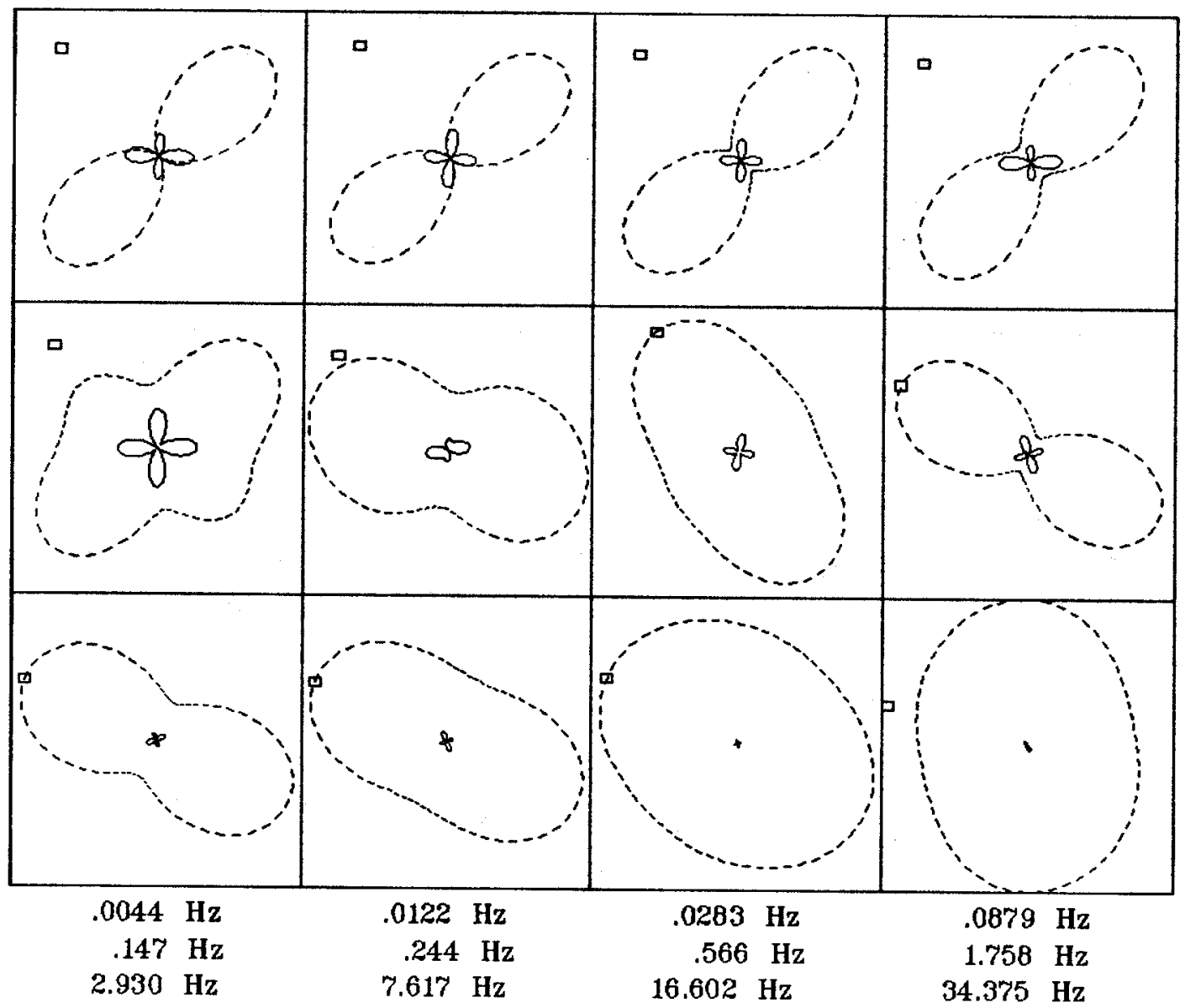

Client:

Remote: Local B

Acquired: 10:1 Aug 01, 1998 Survey Co:USGS GD-MRP Denver
Rotation:

Filename: hr34.avg

Channels: Ch1 Ch2 Ch3 Ch4 Ch5 Ch8 Ch9 Plotted: 13:43 Dec 07, 2000

$<$ EMI - ElectroMagnetic Instruments > 


\section{APPARENT RESISTIVITY}

Humboldt River Line 1

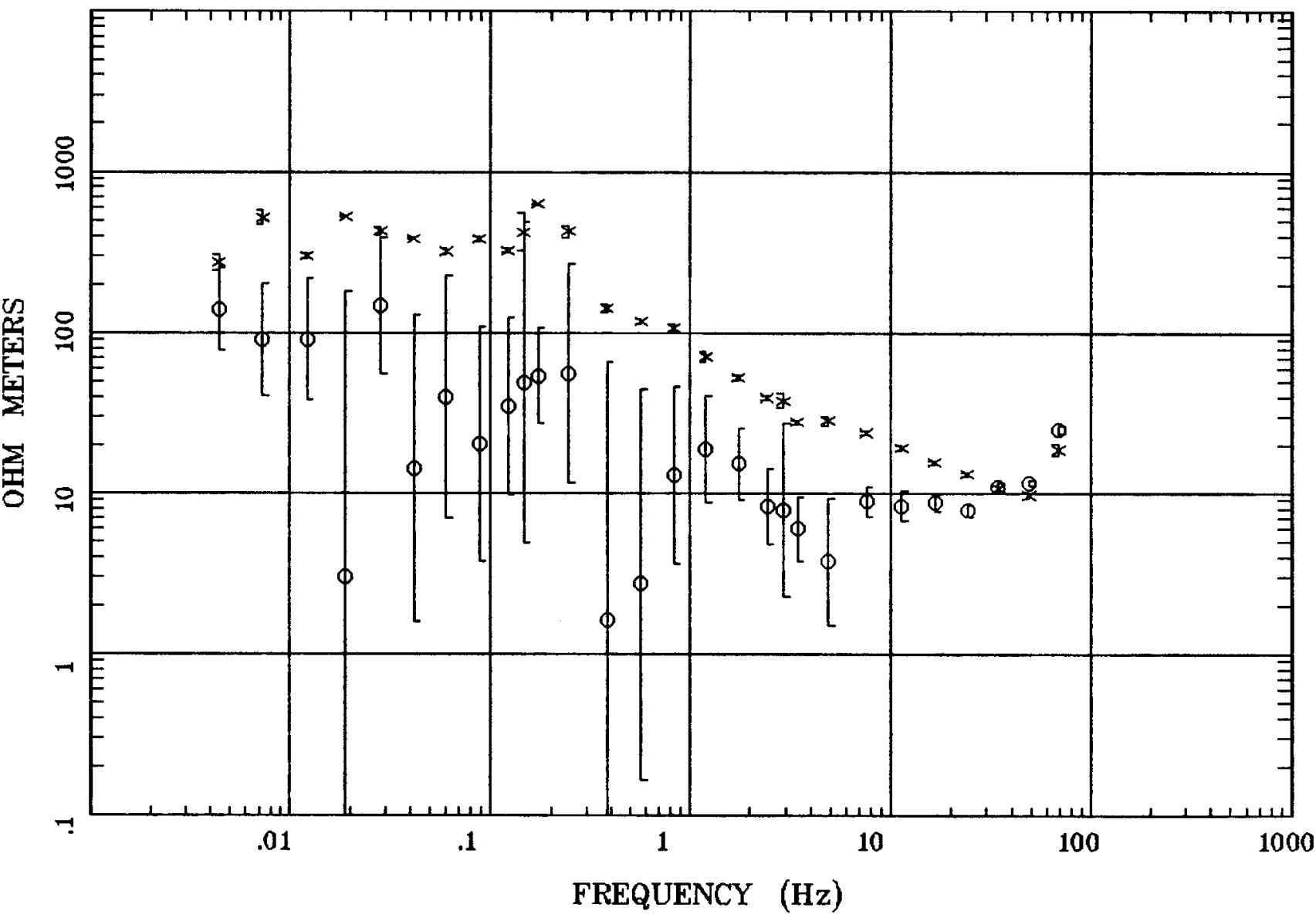

Client:

Remote: none

Acquired: 13:4 Aug 01, 1998

Survey Ca:USGS GD-MRP Denver
Rotation:

Filename: hr37a.avg

Channels: Ch1 Ch2 Ch3 Ch4 Ch5 Ch3 Ch4 Plotted: 13:49 Dec 07, 2000

< EMI - ElectroMagnetic Instruments > 


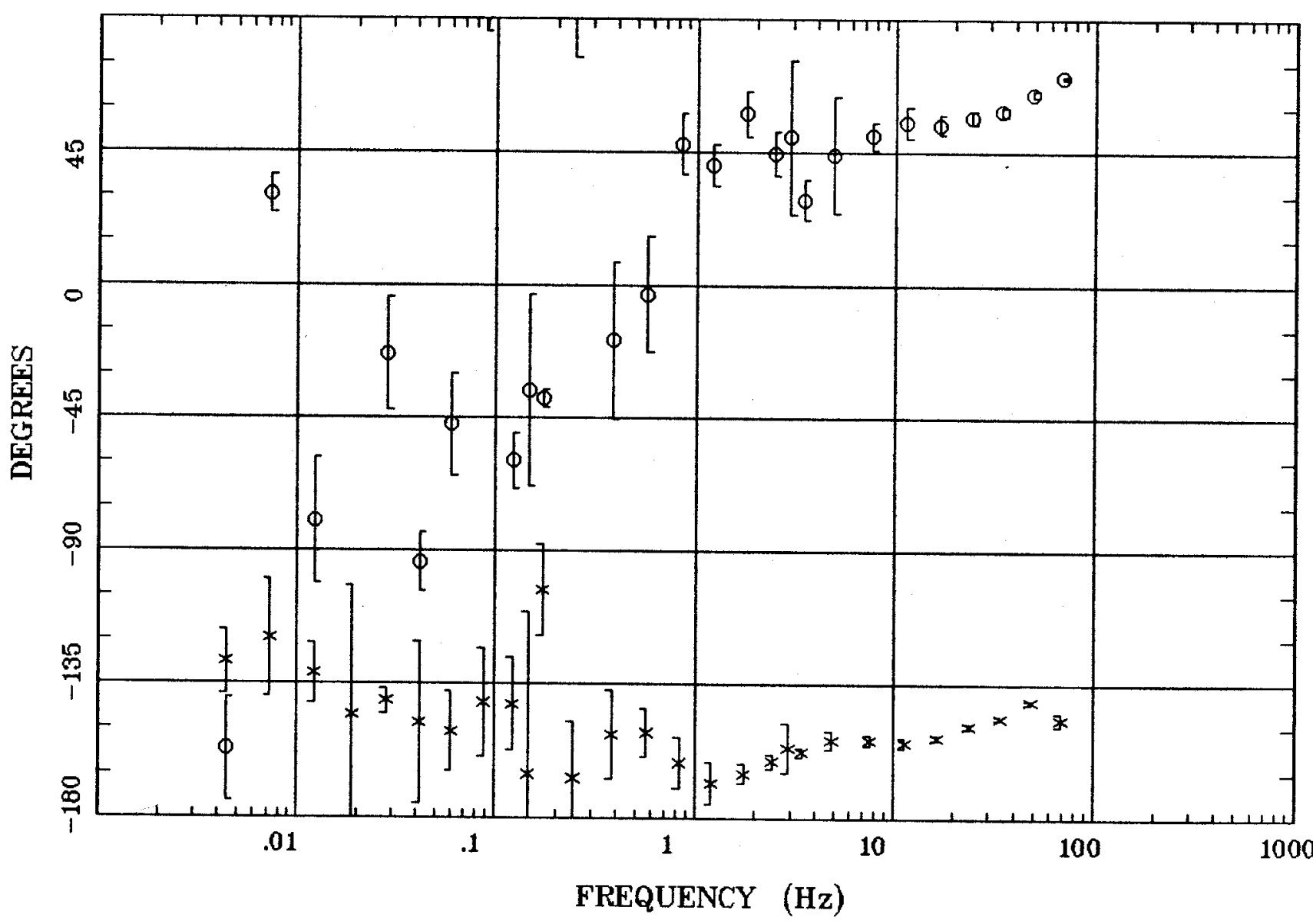

Client:

Remote: none

Acquired: 13:4 Aug 01, 1998

Survey Co:USGS GD-MRP Denver
Rotation:

Filename: hr37a.avg

Channels: Ch1 Ch2 Ch3 Ch4 Ch5 Ch3 Ch4

Plotted: 13:49 Dec 07, 2000

< EMI - ElectroMagnetic Instruments > 


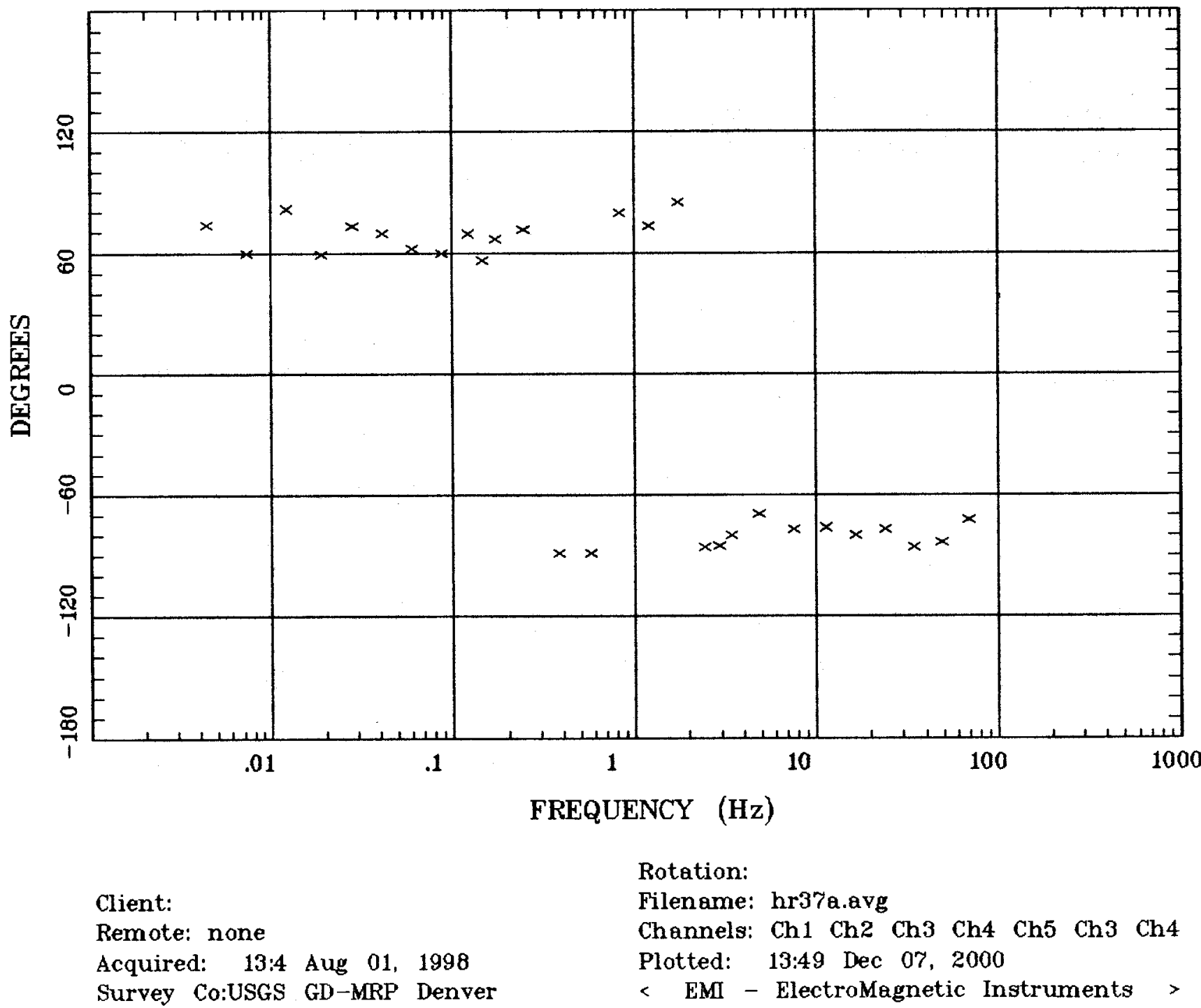




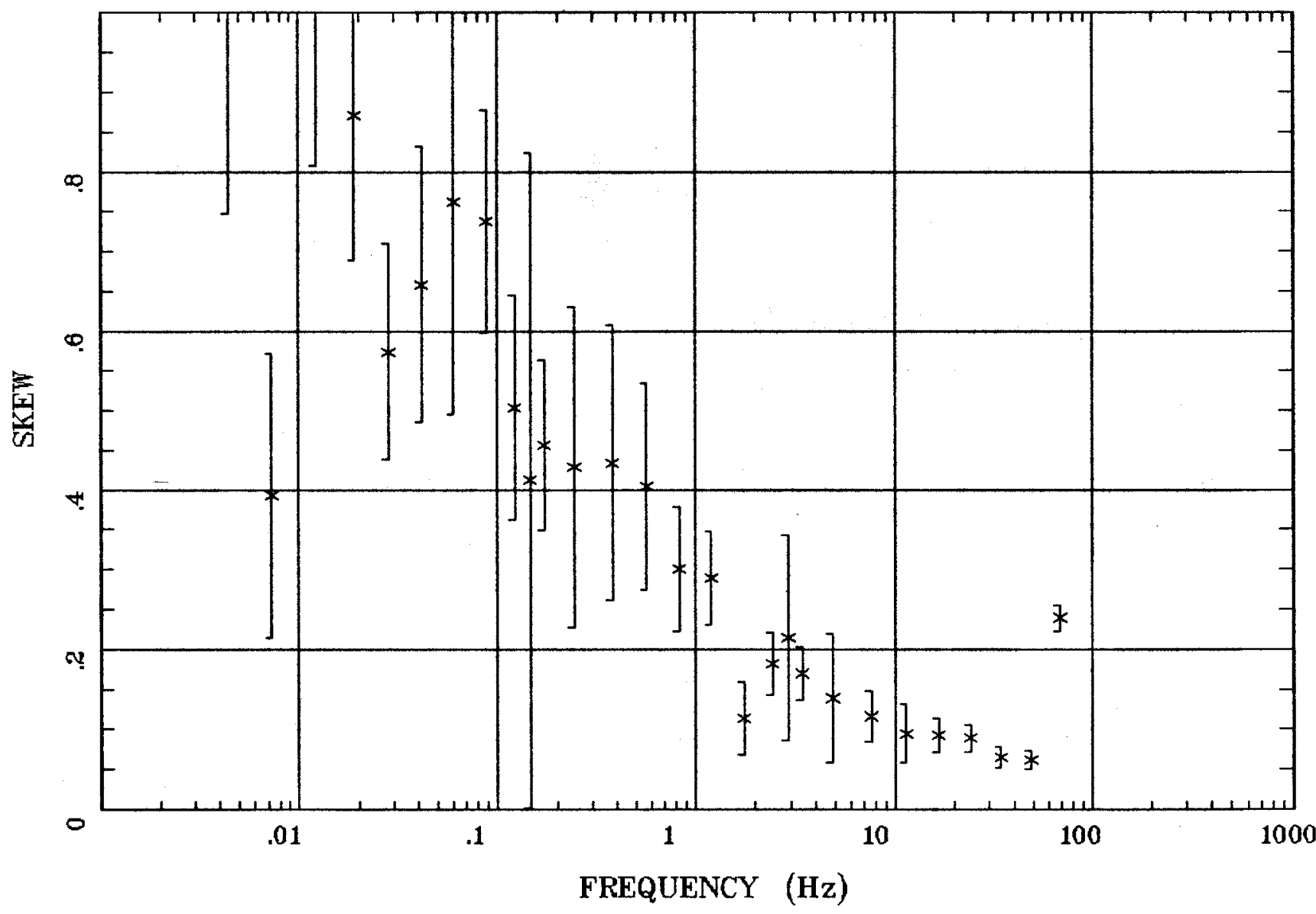

Client:

Remote: none

Acquired: 13:4 Aug 01, 1998

Survey Co:USGS GD-MRP Denver

\section{Rotation:}

Filename: hr37a.avg

Channels: Ch1 Ch2 Ch3 Ch4 Ch5 Ch3 Ch4

Plotted: 13:49 Dec 07, 2000

< EMI - ElectroMagnetic Instruments 




Client:

Remote: none

Acquired: 13:4 Aug 01, 1998

Survey Co:USGS GD-MRP Denver
Rotation:

Filename: hr37a.avg

Channels: Ch1 Ch2 Ch3 Ch4 Ch5 Ch3 Ch4

Plotted: 13:49 Dec 07, 2000

< EMI - ElectroMagnetic Instruments > 


\section{POLAR PLOTS}

Humboldt River Line $1 \quad$ Station 37

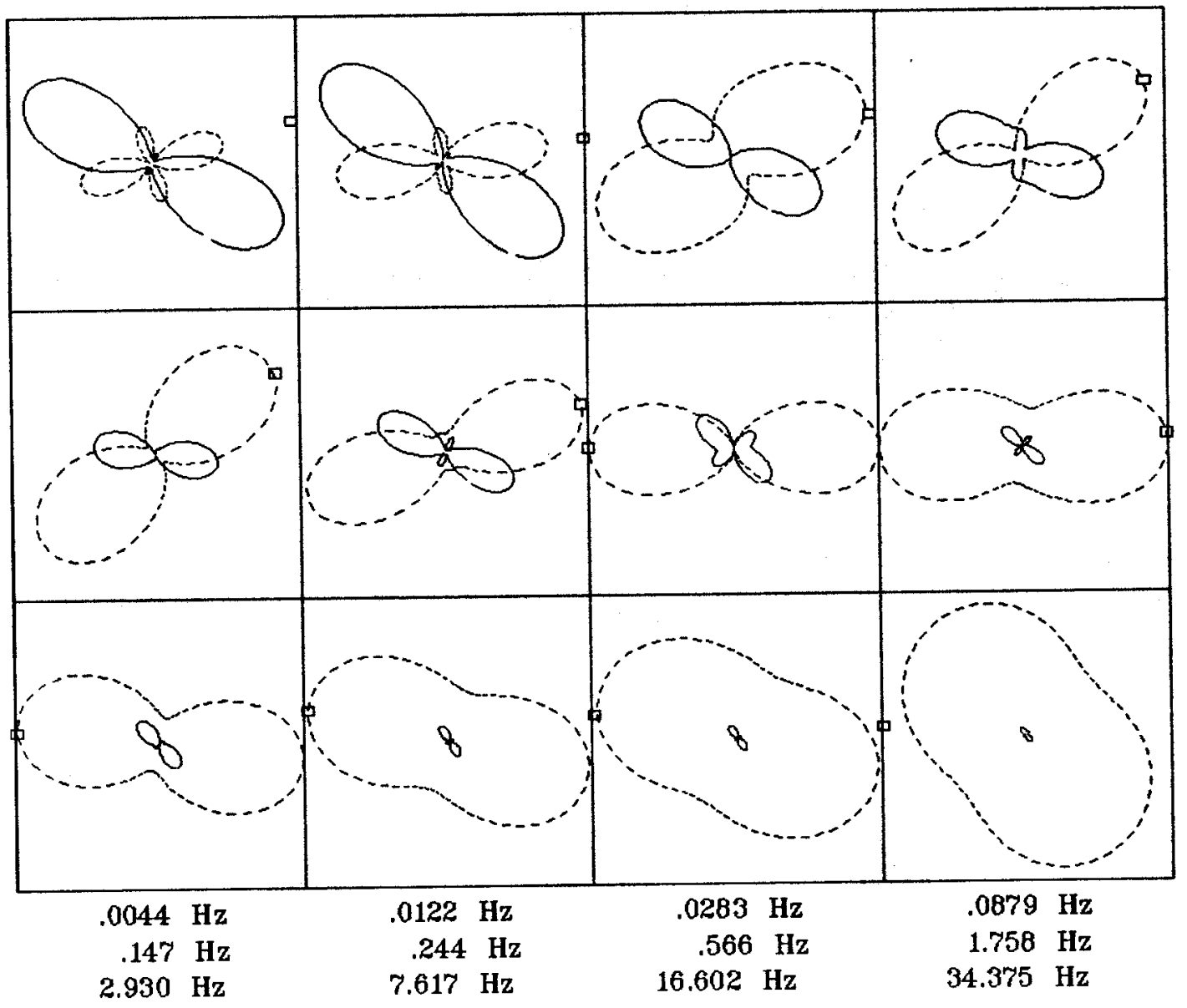

Client:

Remote: none

Acquired: 13:4 Aug 01, 1998

Survey Co:USGS GD-MRP Denver
Rotation:

Filename: hr37a.avg

Channels: Ch1 Ch2 Ch3 Ch4 Ch5 Ch3 Ch4

Plotted: 13:49 Dec 07, 2000

<EMI - ElectroMagnetic Instruments > 


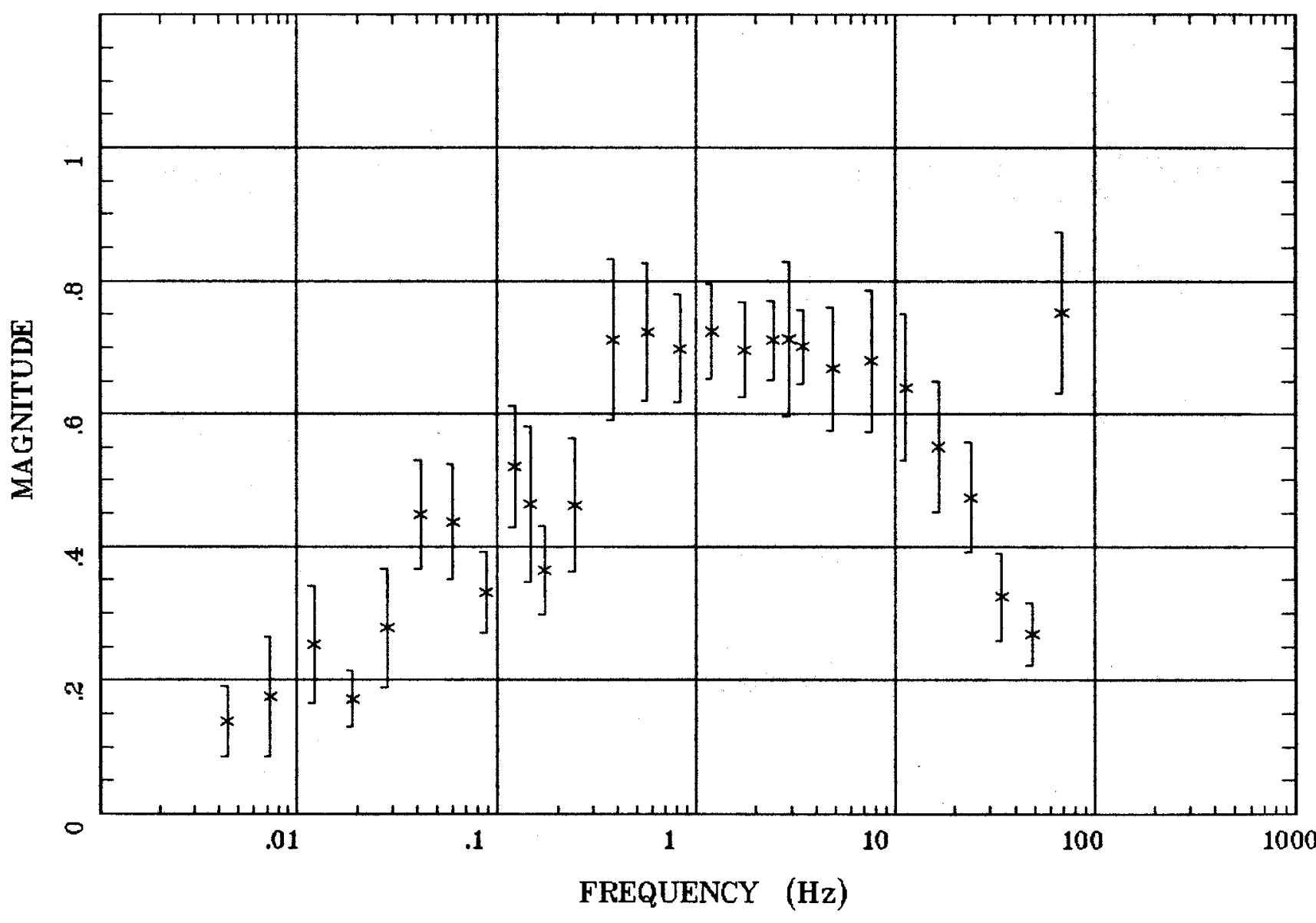

Client:

Remote: none

Acquired: 13:4 Aug 01, 1998 Survey Co:USGS GD-MRP Denver
Rotation:

Filename: hr37a.avg

Channels: Ch1 Ch2 Ch3 Ch4 Ch5 Ch3 Ch4 Plotted: 13:49 Dec 07, 2000

$<$ EMI - ElectroMagnetic Instruments 
Station 37

TIPPER STRIKE

Humboldt River Line 1

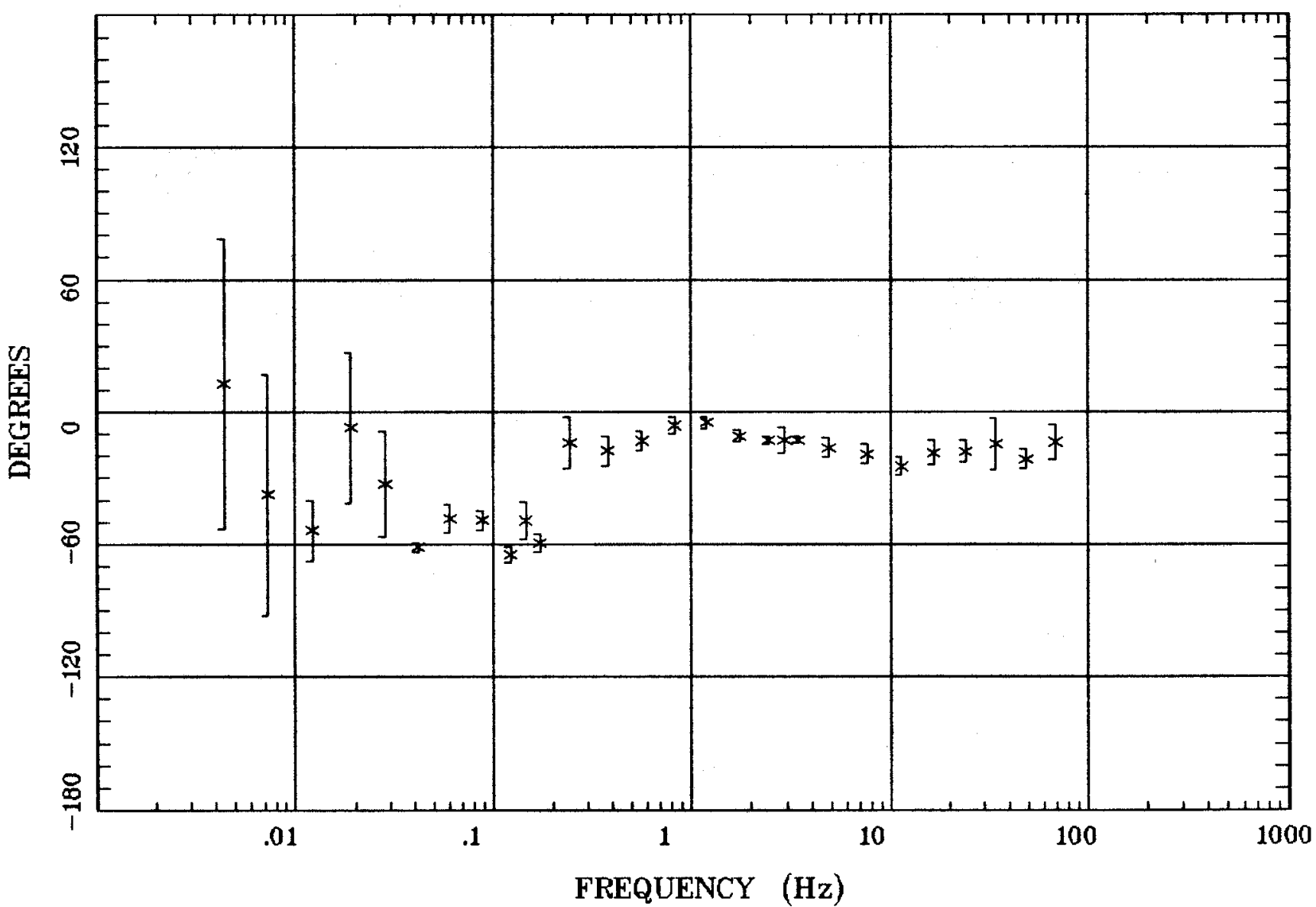

Client:

Remote: none

Acquired: 13:4 Aug 01, 1998

Survey Ca:USGS GD-MRP Denver
Rotation:

Filename: hr37a.avg

Channels: Ch1 Ch2 Ch3 Ch4 Ch5 Ch3 Ch4

Plotted: 13:49 Dec 07, 2000

< EMI - ElectroMagnetic Instruments > 


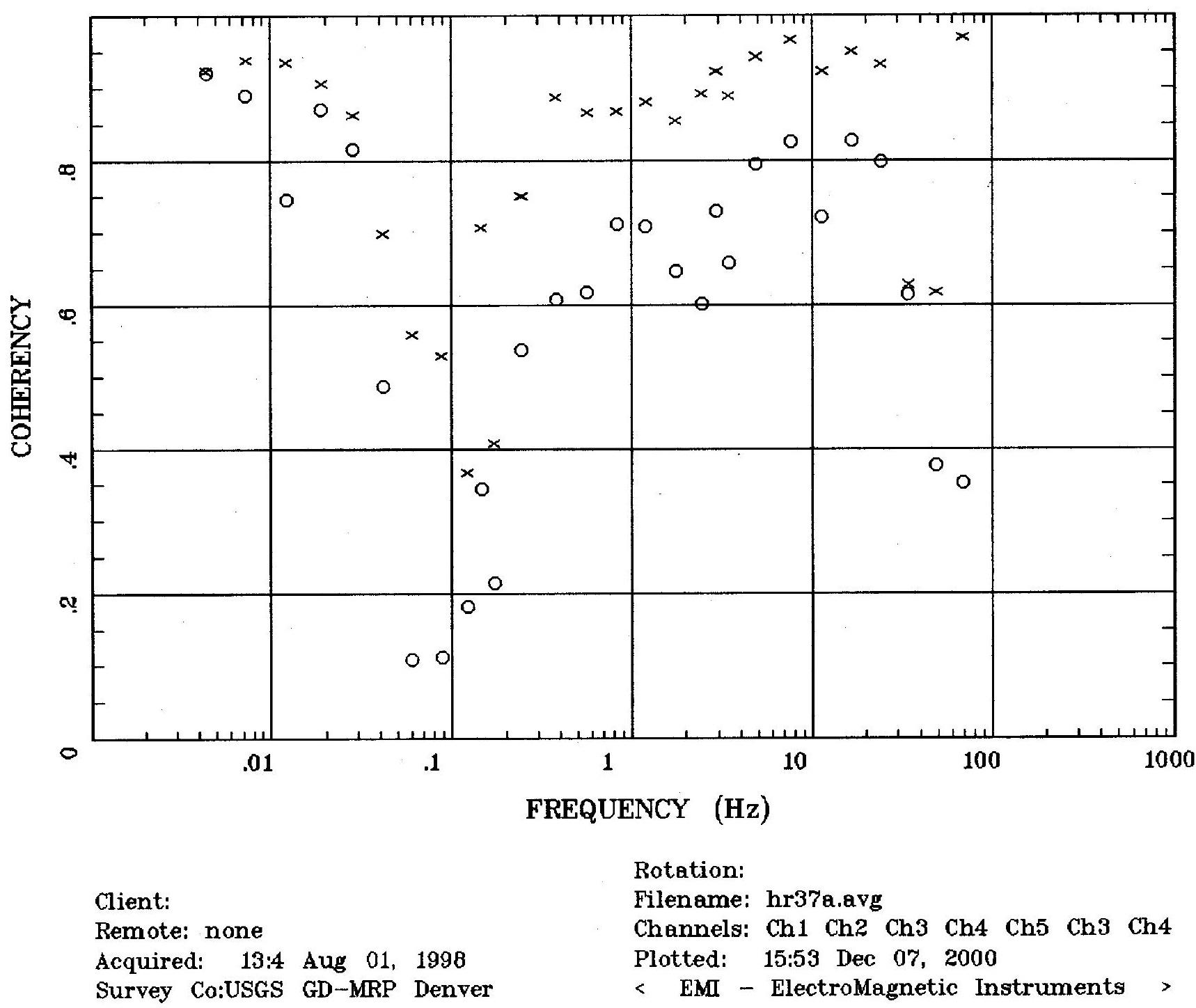




\section{Station 33}

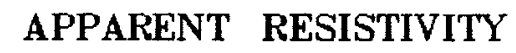

Humboldt River Line 1

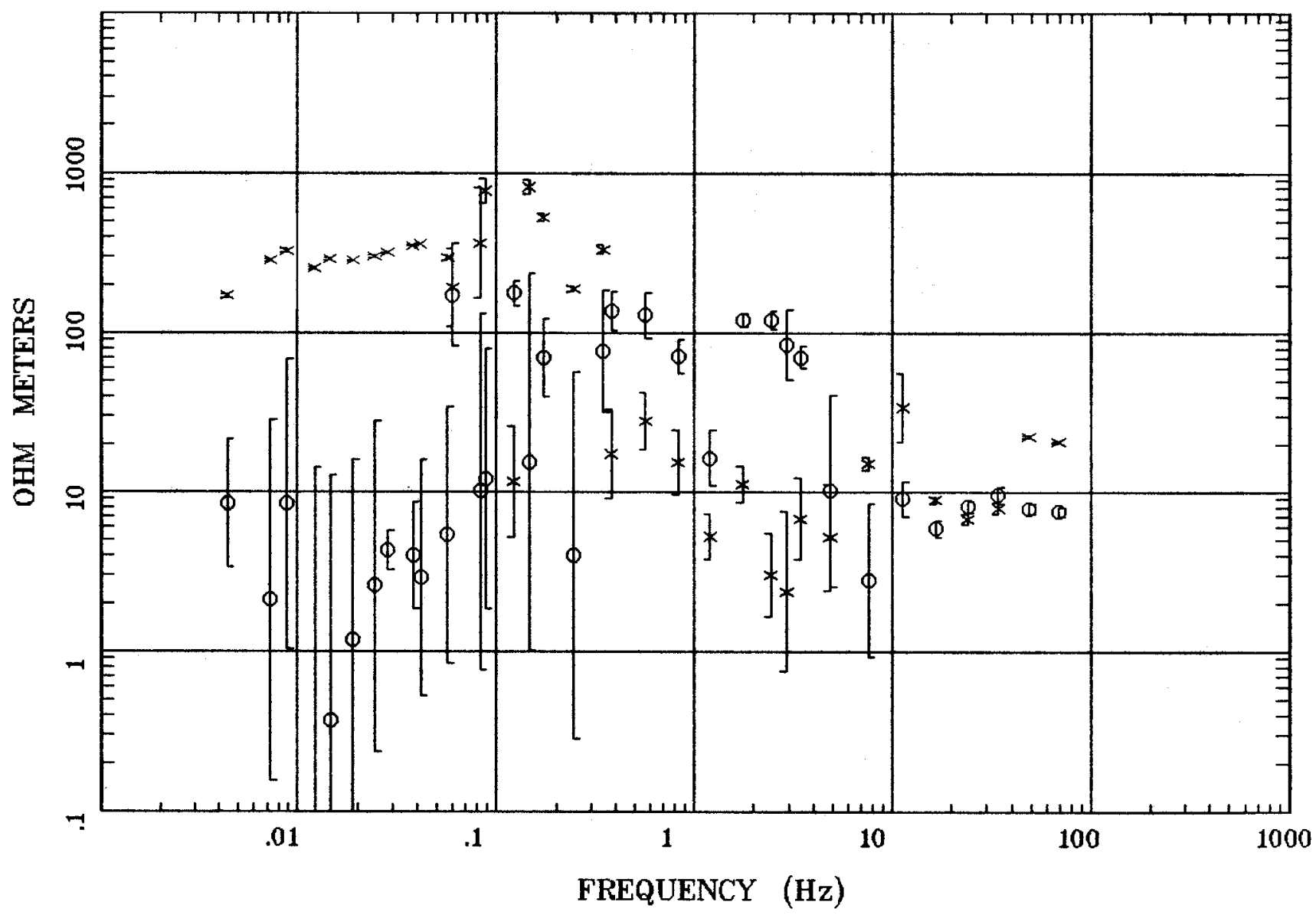

Client:

Remote: Local $B$

Acquired: 10:0 Aug 02, 1998 Survey Co:USGS GD-MRP Denver
Rotation:

Filename: HR33.avg

Channels: Ch1 Ch2 Ch3 Ch4 Ch5 Ch8 Ch9 Plotted: 13:28 Dec 07, 2000

$<$ EMI - ElectroMagnetic Instruments > 
Station 33

Humboldt River Line 1

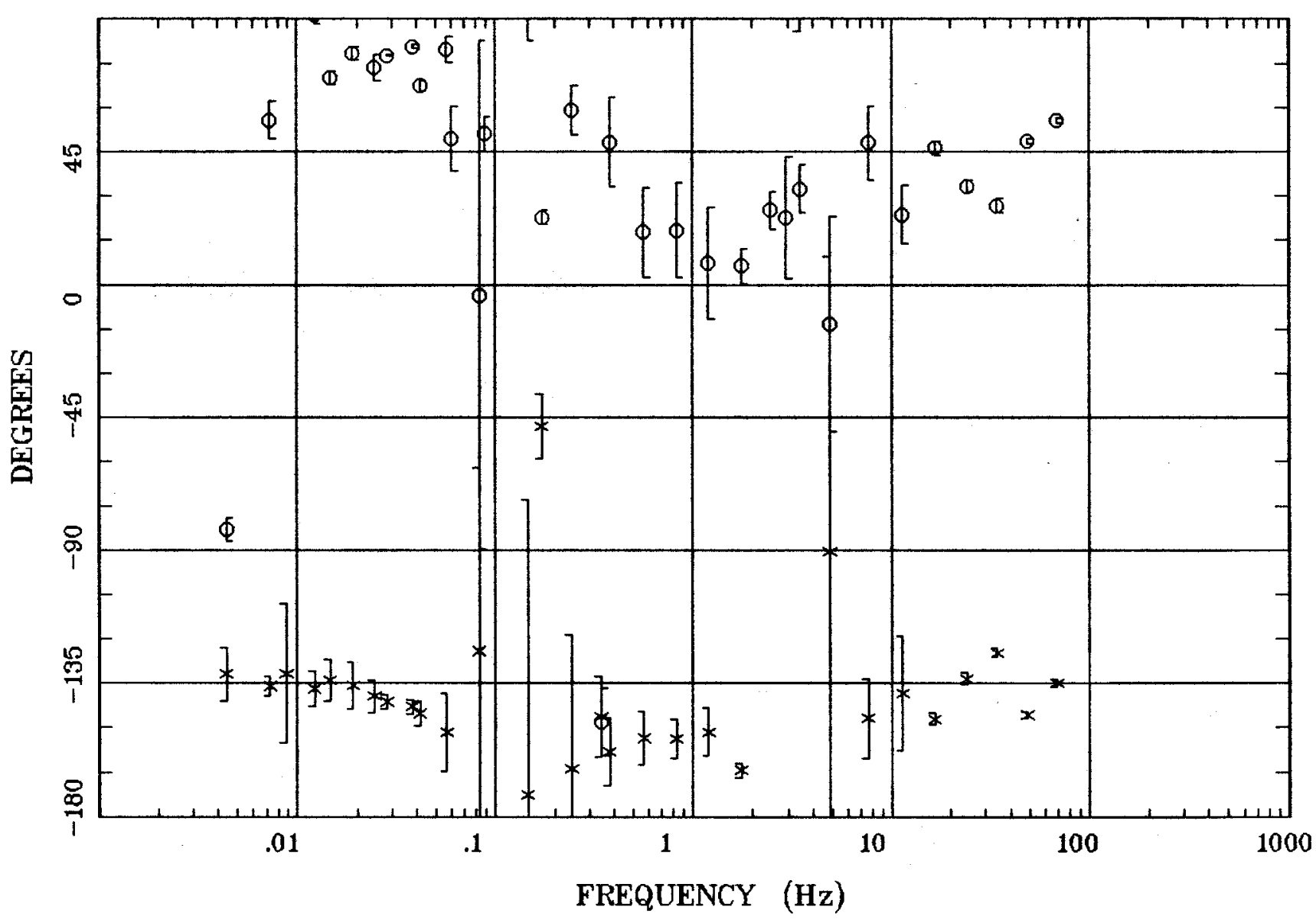

Rotation:

Client:

Filename: HR33.avg

Remote: Local B

Acquired: 10:0 Aug 02, 1998

Channels: Ch1 Ch2 Ch3 Ch4 Ch5 Ch8 Ch9

Survey Co:USGS GD-MRP Denver

Plotted: 13:28 Dec 07, 2000

< EMI - ElectroMagnetic Instruments > 


\section{Station 33}

ROTATION ANGLE

Humboldt River Line 1

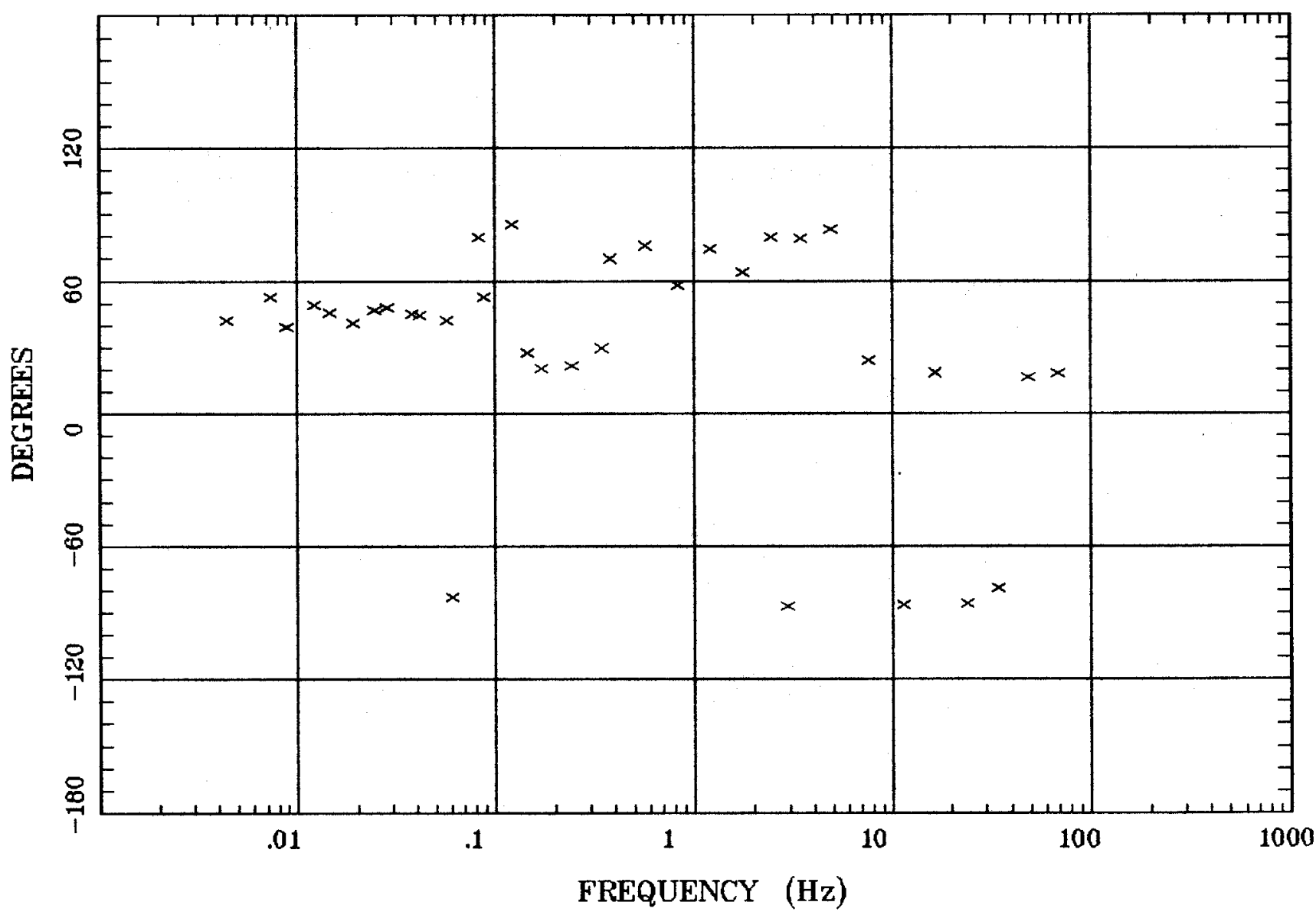

Client:

Remote: Local B

Acquired: 10:0 Aug 02, 1998

Survey Co:USGS GD-MRP Denver
Rotation:

Filename: HR33.avg

Channels: Ch1 Ch2 Ch3 Ch4 Ch5 Ch8 Ch9

Plotted: 13:29 Dec 07, 2000

$<$ EMI - ElectroMagnetic Instruments > 
Humboldt River Line 1



Client:

Remote: Local $B$

Acquired: 10:0 Aug 02, 1998

Survey Co:USGS GD-MRP Denver
Rotation:

Filename: HR33.avg

Channels: Ch1 Ch2 Ch3 Ch4 Ch5 Ch8 Ch9

Plotted: 13:29 Dec 07, 2000

< EMI - ElectroMagnetic Instruments > 
Station 33

E MULT Coh. Humboldt River Line 1

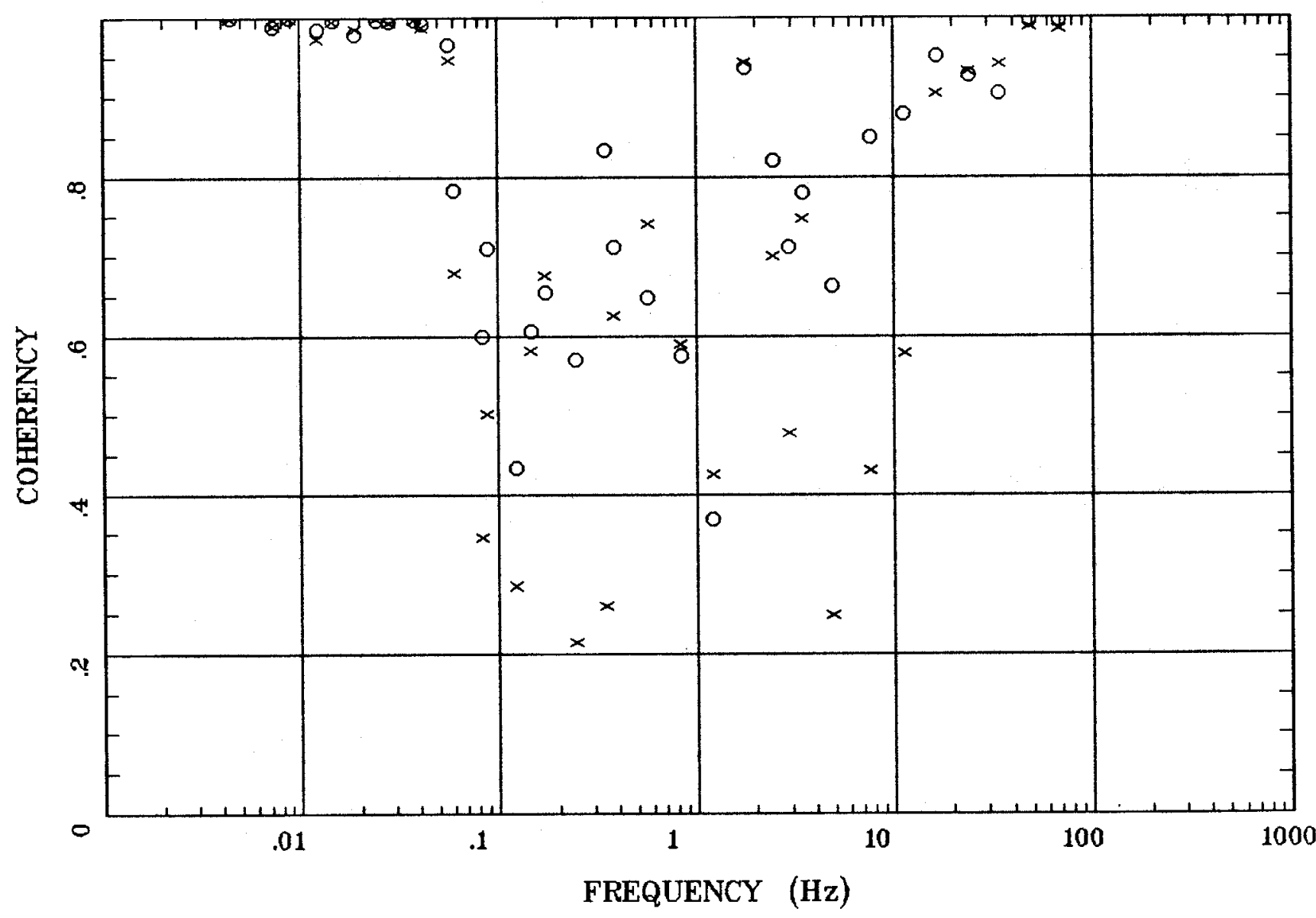

Client:

Remote: Local B

Acquired: 10:0 Aug 02, 1998 Survey Co:USGS GD-MRP Denver
Rotation:

Filename: HR33.avg

Channels: Ch1 Ch2 Ch3 Ch4 Ch5 Ch8 Ch9 Plotted: 13:29 Dec 07, 2000

$<$ EMI - ElectroMagnetic Instruments 
Station 33

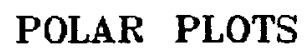

Humboldt River Line 1

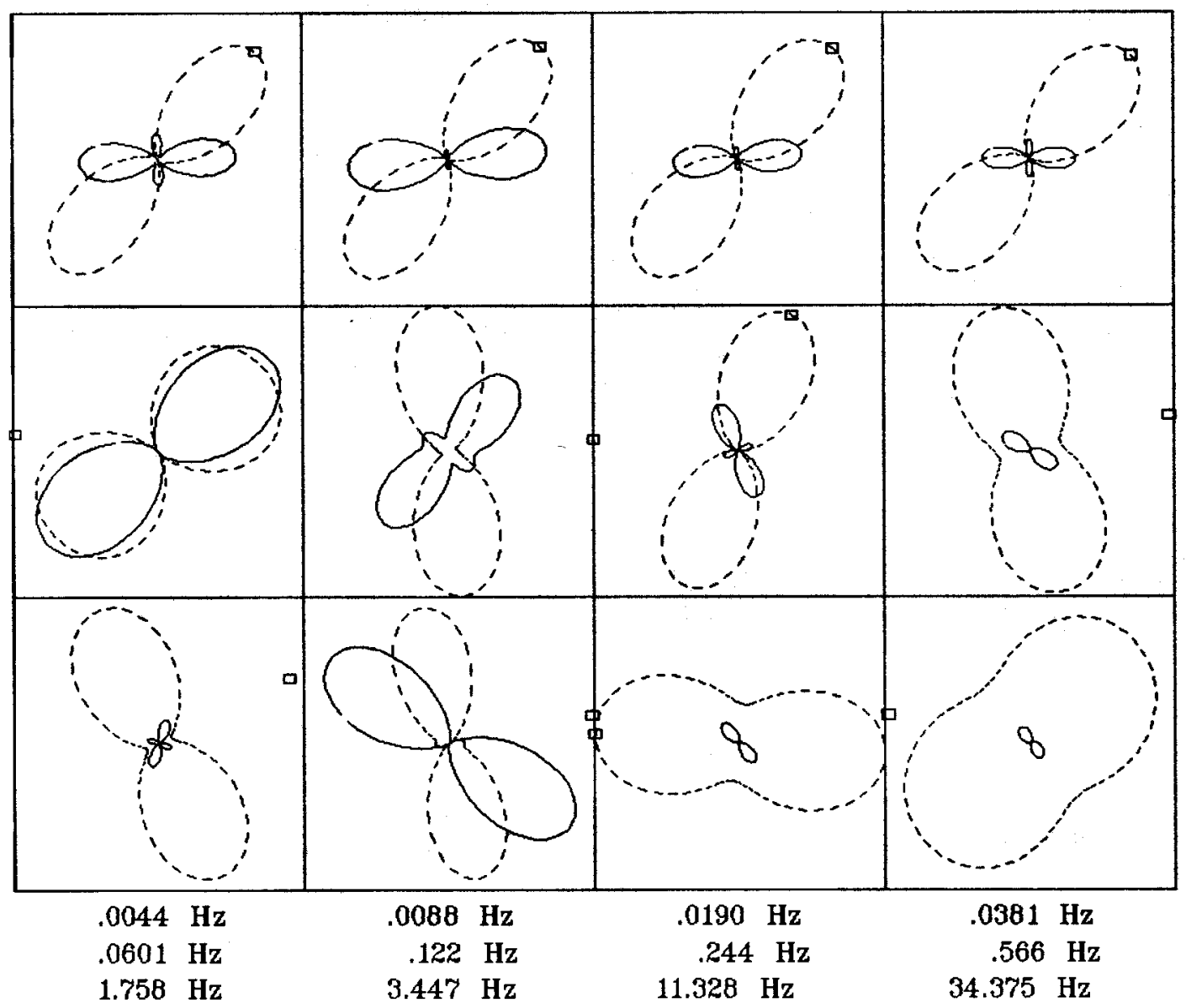

Rotation:

Client:

Remote: Local B

Acquired: 10:0 Aug 02, 1998

Survey Co:USGS GD-MRP Denver
Filename: HR33.avg

Channels: Ch1 Ch2 Ch3 Ch4 Ch5 Ch8 Ch9

Plotted: 13:29 Dec 07, 2000

$<$ EMI - ElectroMagnetic Instruments > 


\section{Station 32}

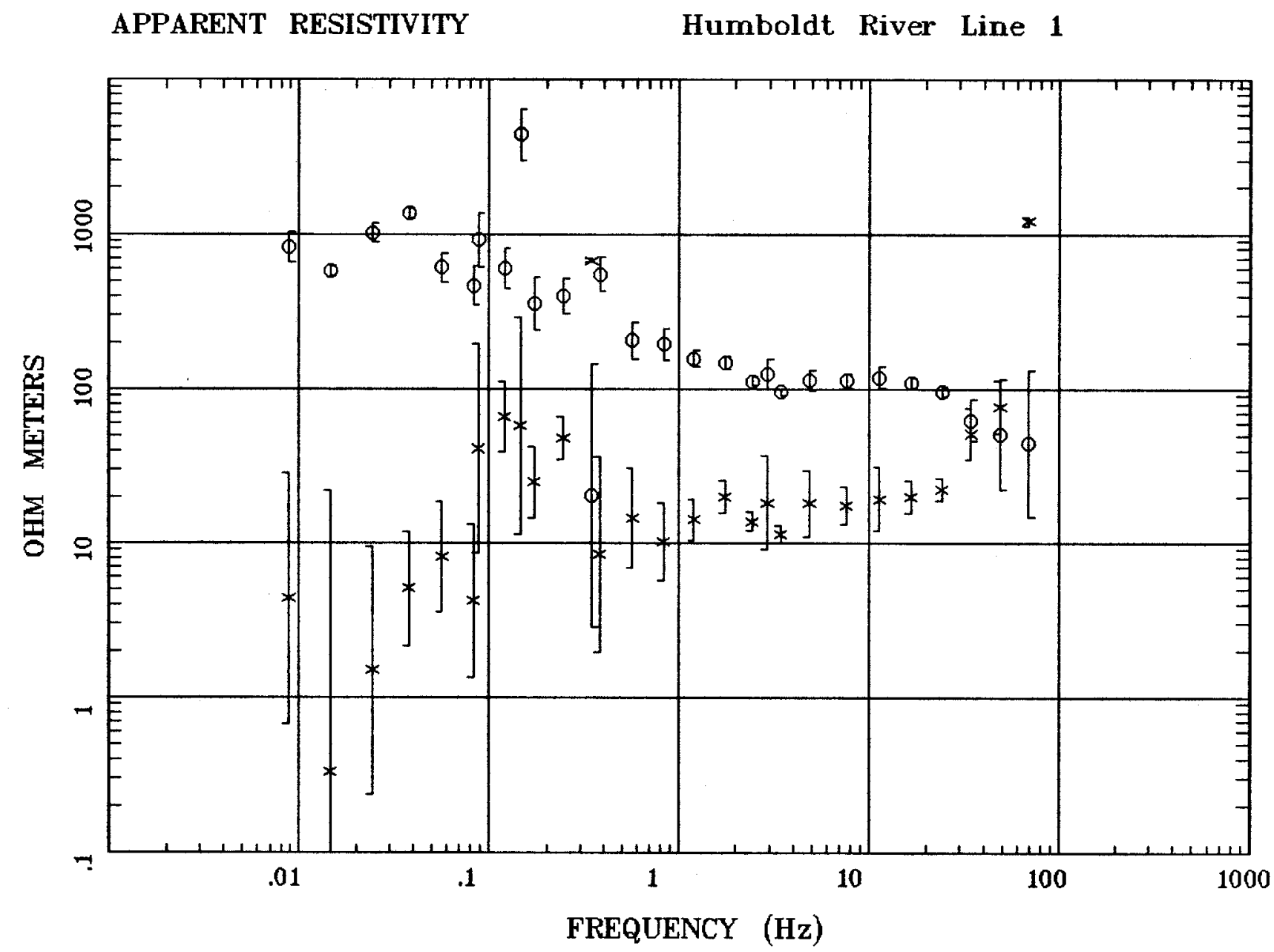

Client:

Remote: Local B

Acquired: 14:1 Aug 02, 1998 Survey Co:USGS GD-MRP Denver
Rotation:

Filename: HR32.avg

Channels: Ch1 Ch2 Ch3 Ch4 Ch5 Ch8 Ch9

Plotted: 13:22 Dec 07, 2000

< EMI - ElectroMagnetic Instruments 
Humboldt River Line 1

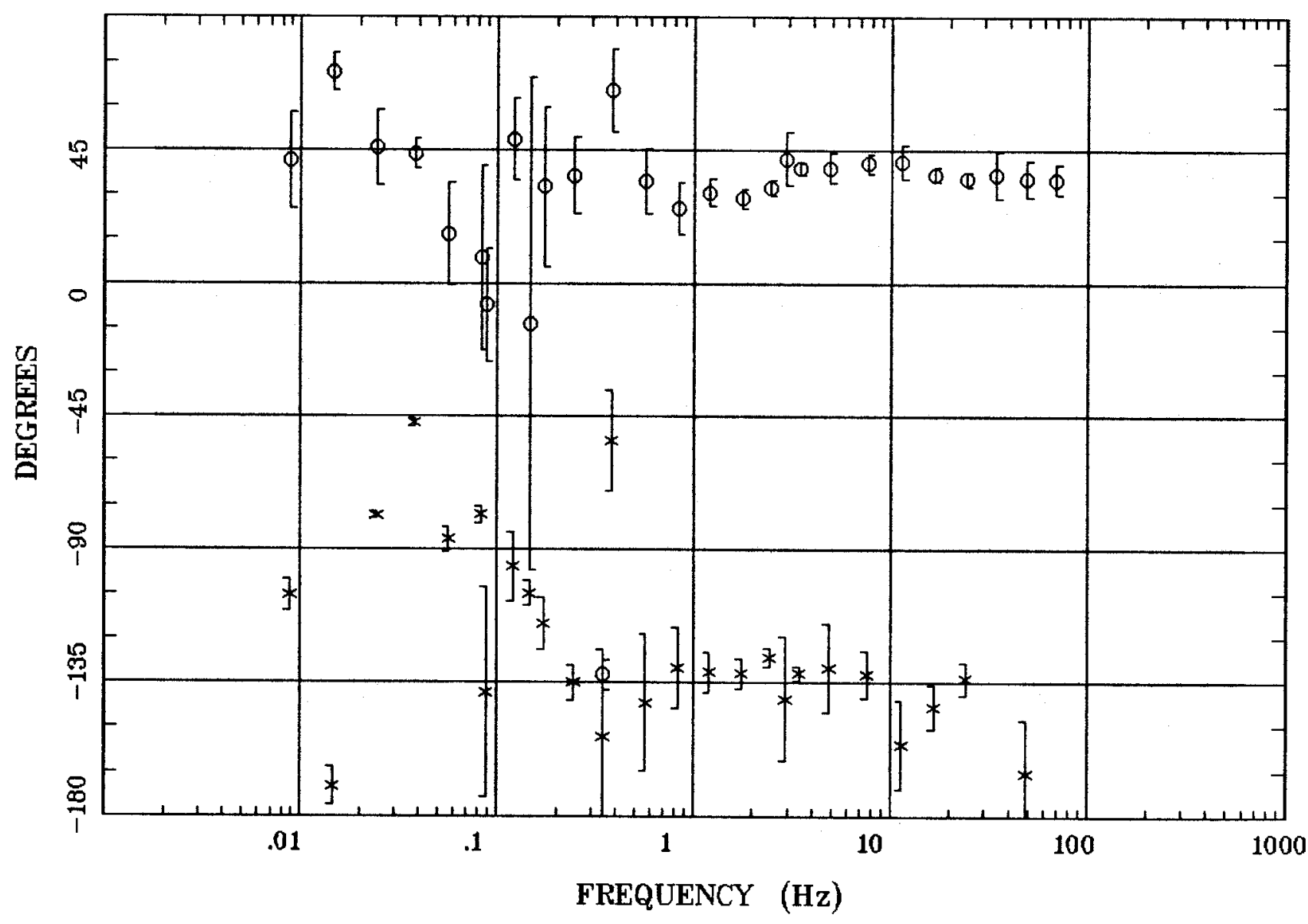

Client:

Remote: Local $B$

Acquired: 14:1 Aug 02, 1998 Survey Co:USGS GD-MRP Denver
Rotation:

Filename: HR32.avg

Channels: Ch1 Ch2 Ch3 Ch4 Ch5 Ch8 Ch9

Plotted: 13:22 Dec 07, 2000

< EMI - ElectroMagnetic Instruments > 


\section{ROTATION ANGLE}

Humboldt River Line 1

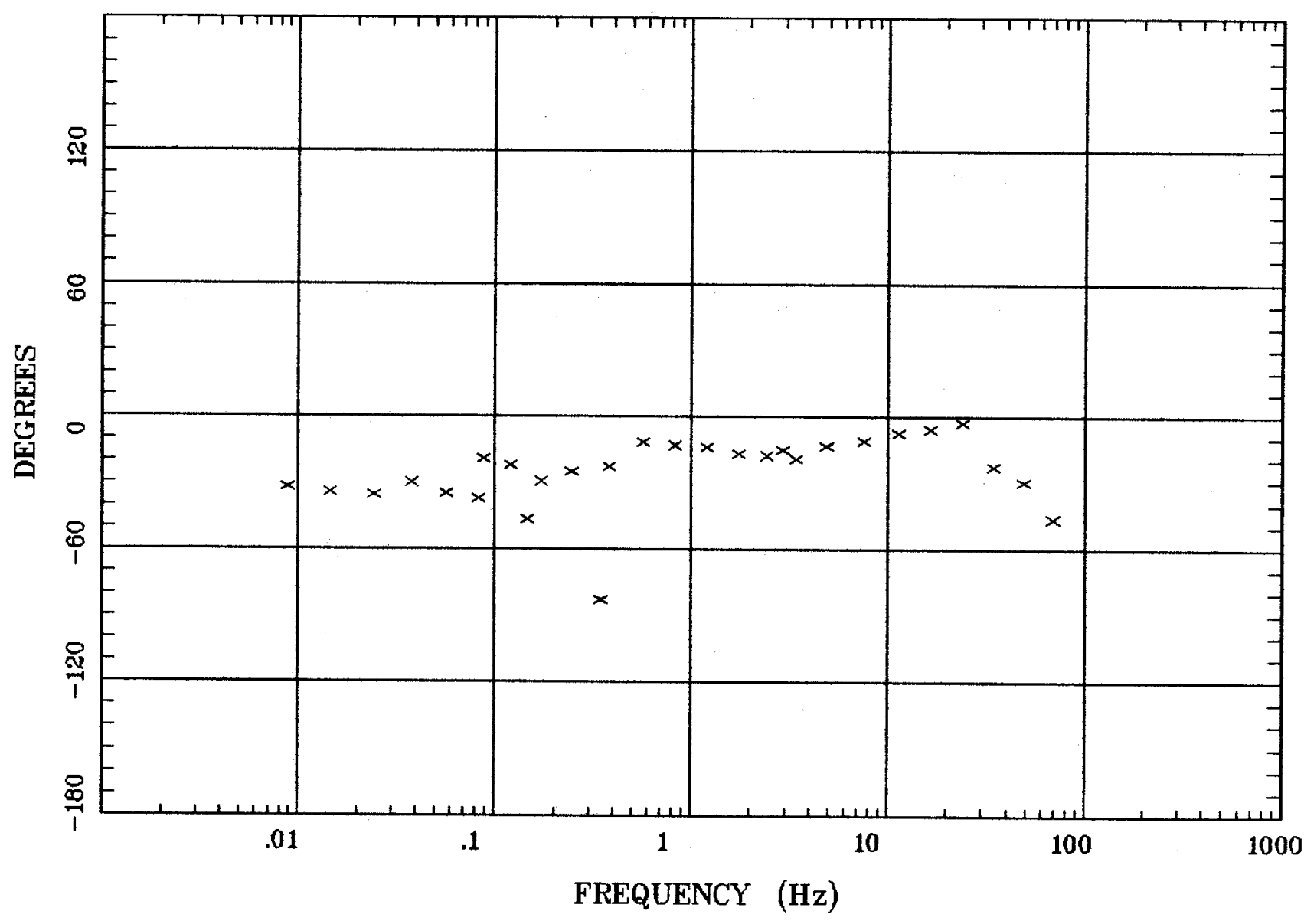

Client:

Remote: Local B

Acquired: 14:1 Aug 02, 1998

Survey Co:USGS GD-MRP Denver
Rotation:

Filename: HR32.avg

Channels: Ch1 Ch2 Ch3 Ch4 Ch5 Ch8 Ch9

Plotted: 13:22 Dec 07, 2000

$<$ EMI - ElectroMagnetic Instruments > 
Station 32

IMPEDANCE SKEW



Client:

Remote: Local $B$

Acquired: 14:1 Aug 02, 1998 Survey Co:USGS GD-MRP Denver
Rotation:

Filename: HR32.avg

Channels: Ch1 Ch2 Ch3 Ch4 Ch5 Ch8 Ch9

Plotted: 13:22 Dec 07, 2000

< EMI - ElectroMagnetic Instruments 


\section{Station 32}

E MULT Coh.

Humboldt River Line 1

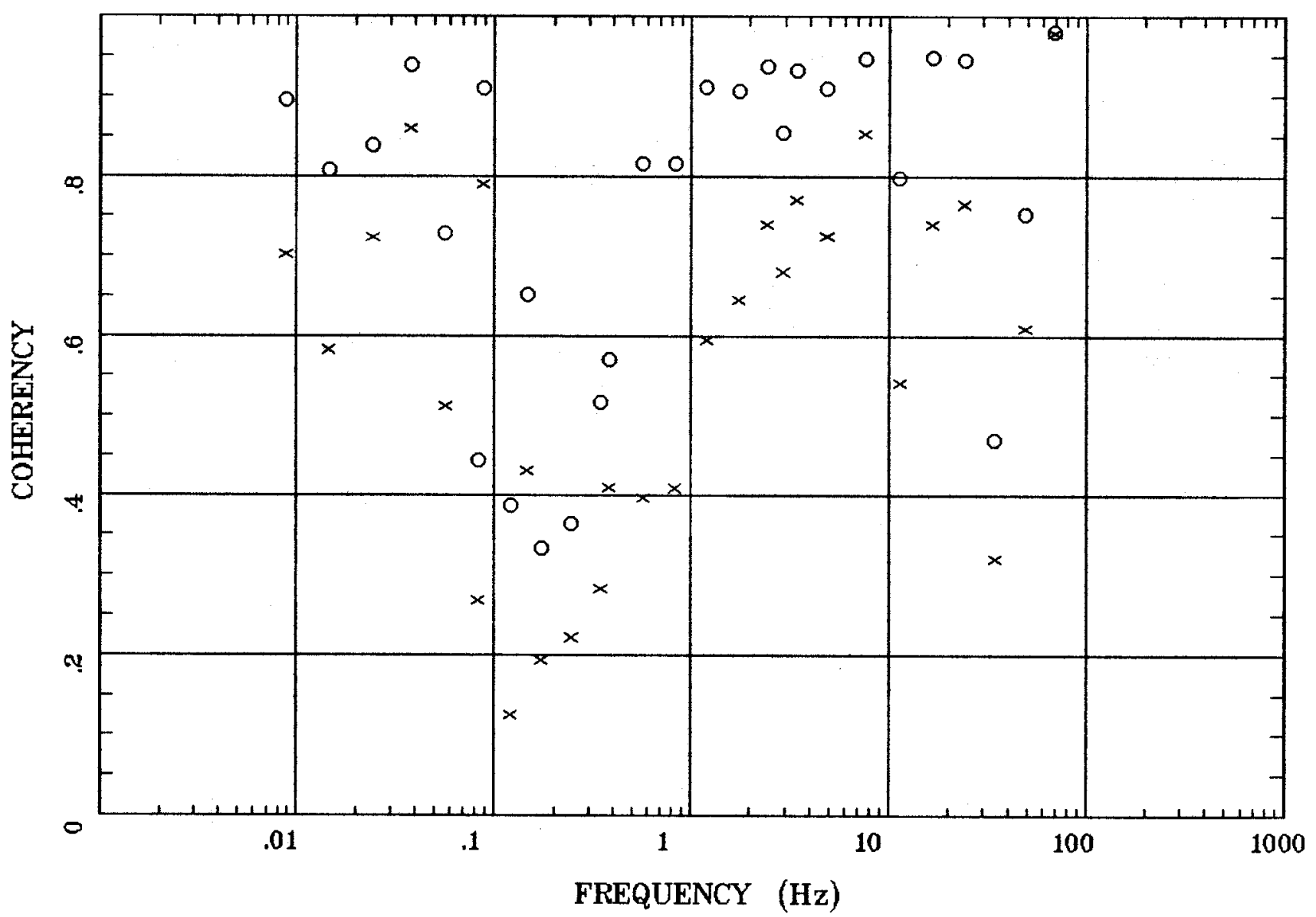

Client:

Remote: Local $\mathbf{B}$

Acquired: 14:1 Aug 02, 1998 Survey Co:USGS GD-MRP Denver
Rotation:

Filename: HR32.avg

Channels: Ch1 Ch2 Ch3 Ch4 Ch5 Ch8 Ch9

Plotted: 13:22 Dec 07, 2000

< EMI - ElectroMagnetic Instruments > 


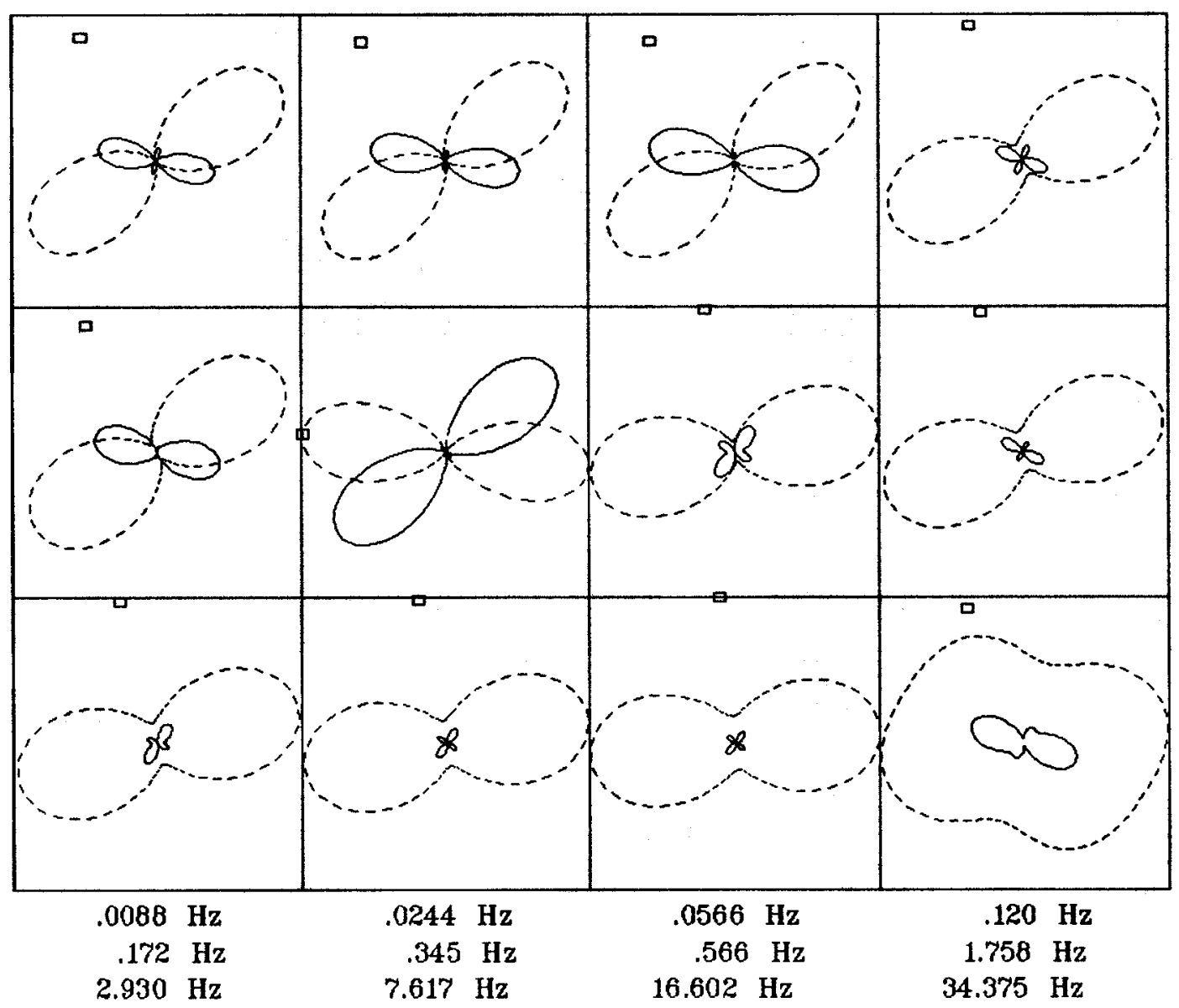

Rotation:

Client:

Remote: Local B

Acquired: 14:1 Aug 02, 1998 Survey Co:USGS GD-MRP Denver
Filename: HR32.avg

Channels: Ch1 Ch2 Ch3 ch4 Ch5 Ch8 Ch9 Plotted: 13:22 Dec 07, 2000

< EMI - ElectroMagnetic Instruments > 
APPARENT RESISTIVITY

Humboldt River Line 1

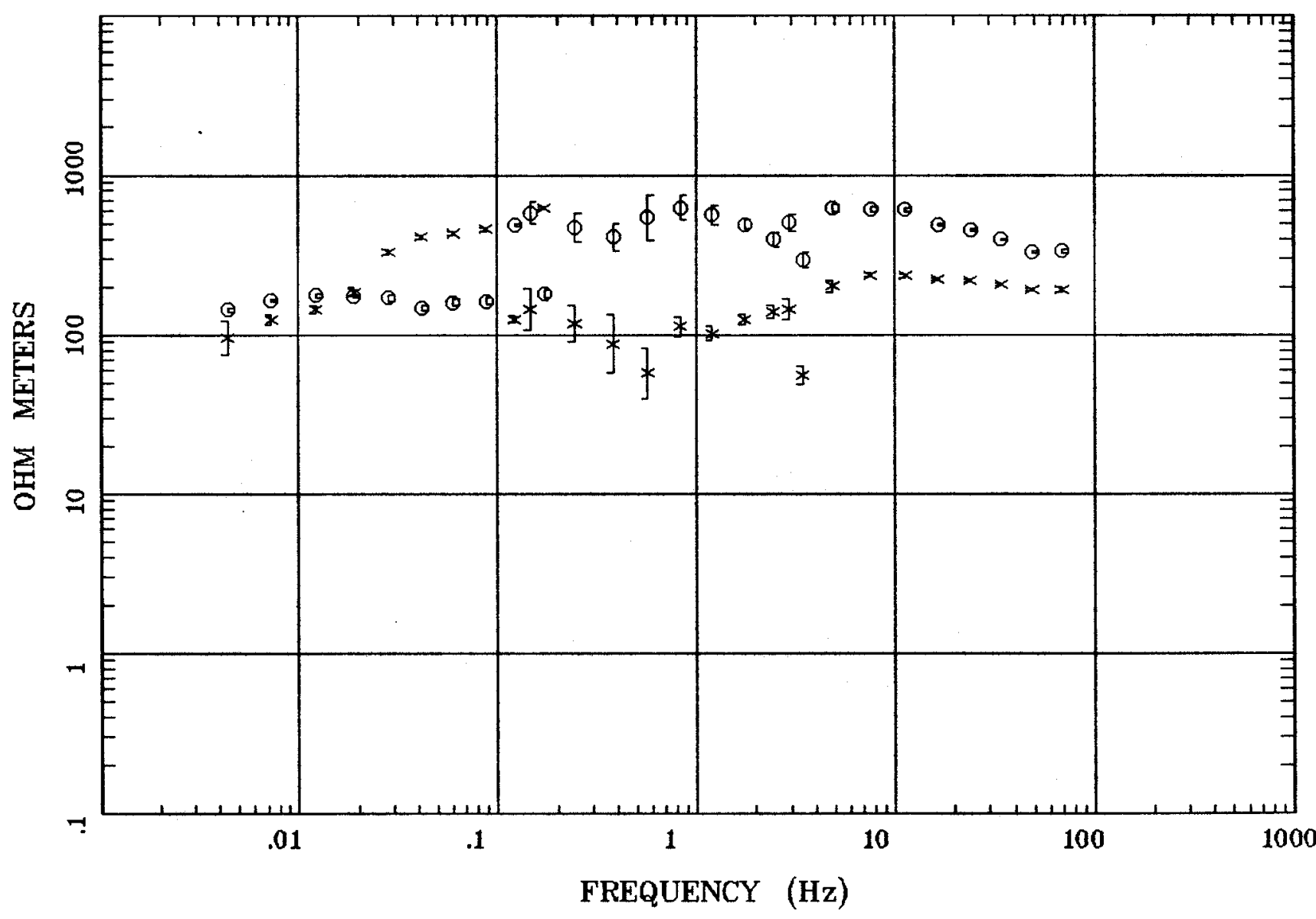

Client:

Remote: Local B

Acquired: 10:2 Jul 31, 1998

Survey Co:USGS GD-MRP Denver
Rotation:

Filename: hr35.avg

Channels: Ch1 Ch2 Ch3 Ch4 Ch5 Ch8 Ch9 Plotted: 13:44 Dec 07, 2000

< EMI - ElectroMagnetic Instruments > 


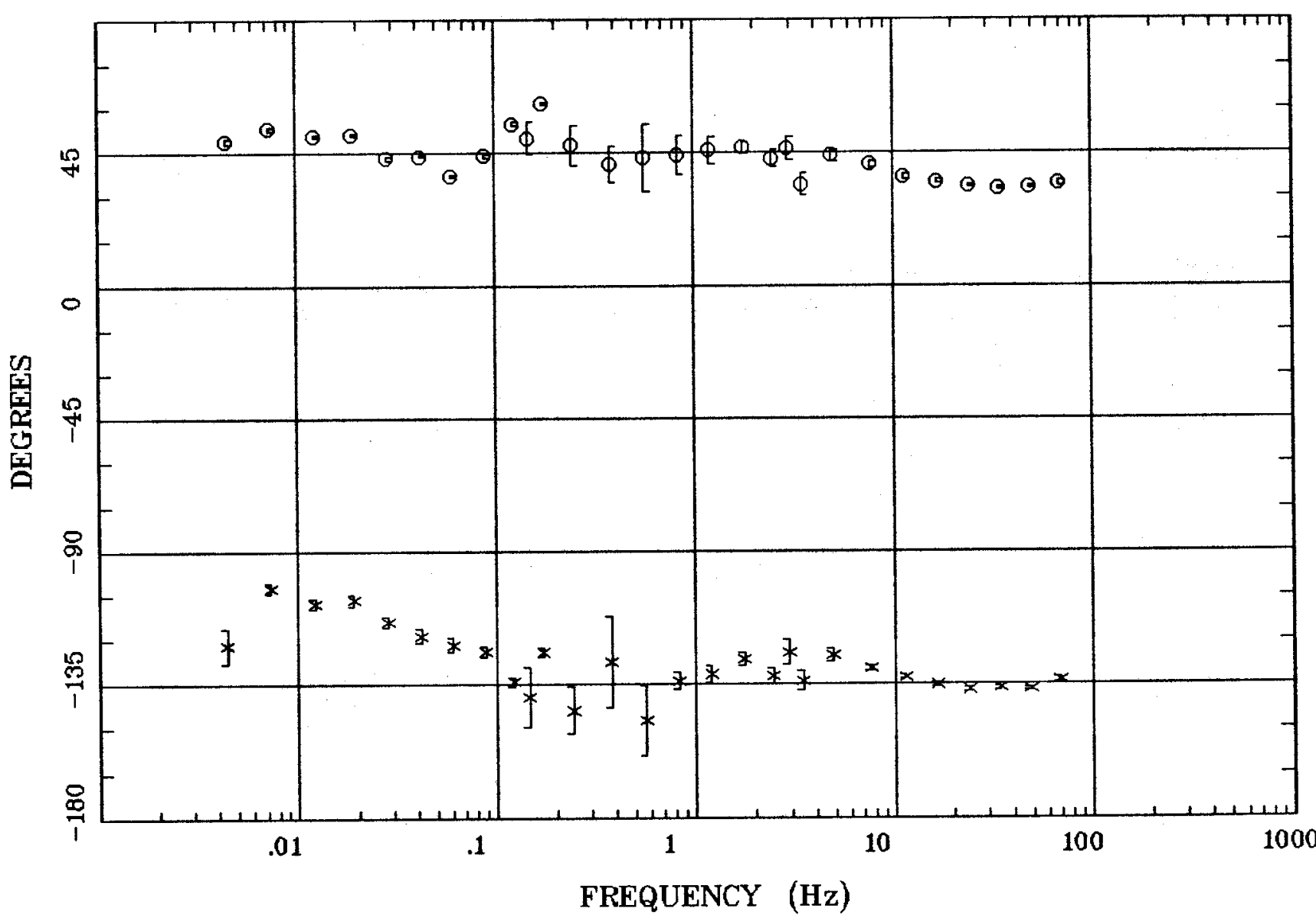

Client:

Remote: Local B

Acquired: 10:2 Jul 31, 1998 Survey Co:USGS GD-MRP Denver
Rotation:

Filename: hr35.avg

Channels: Ch1 Ch2 Ch3 Ch4 Ch5 Ch8 Ch9

Plotted: 13:44 Dec 07, 2000

$<$ EMI - ElectroMagnetic Instruments 


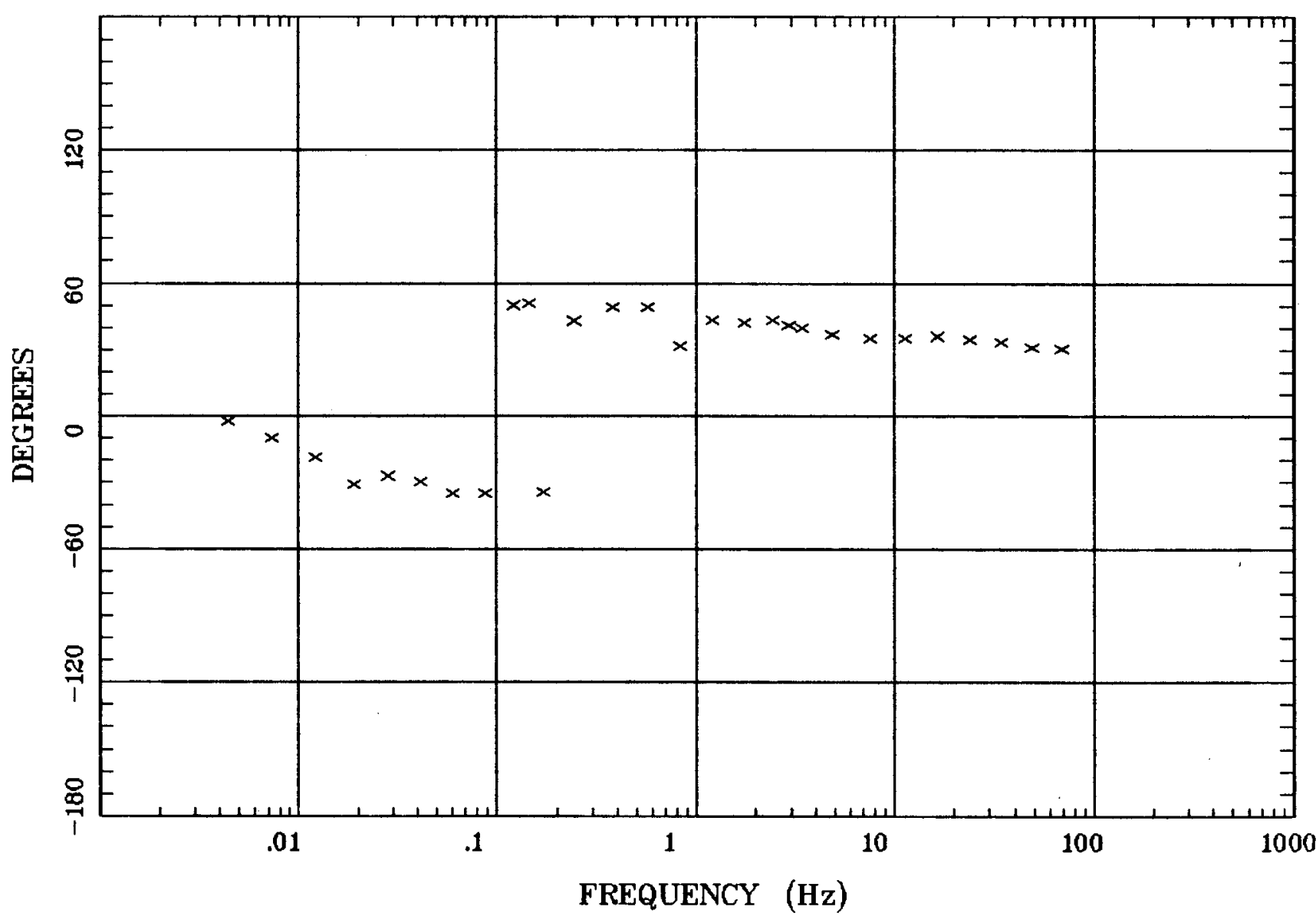

Client:

Remote: Local B

Acquired: 10:2 Jul 31, 1998

Survey Co:USGS GD-MRP Denver

Rotation:

Filename: hr35.avg

Channels: Ch1 Ch2 Ch3 Ch4 Ch5 Ch8 Ch9

Plotted: 13:44 Dec 07, 2000

$<$ EMI - ElectroMagnetic Instruments 
Station 35

IMPEDANCE SKEW

Humboldt River Line 1



Client:

Remote: Local B

Acquired: $10: 2$ Jul 31,1998

Survey Co:USGS GD-MRP Denver

Rotation:

Filename: hr35.avg

Channels: Ch1 Ch2 Ch3 Ch4 Ch5 Ch8 Ch9

Plotted: 13:44 Dec 07, 2000

< EMI - ElectroMagnetic Instruments 


\section{Station 35}

E MULT Coh.

Humboldt River Line 1

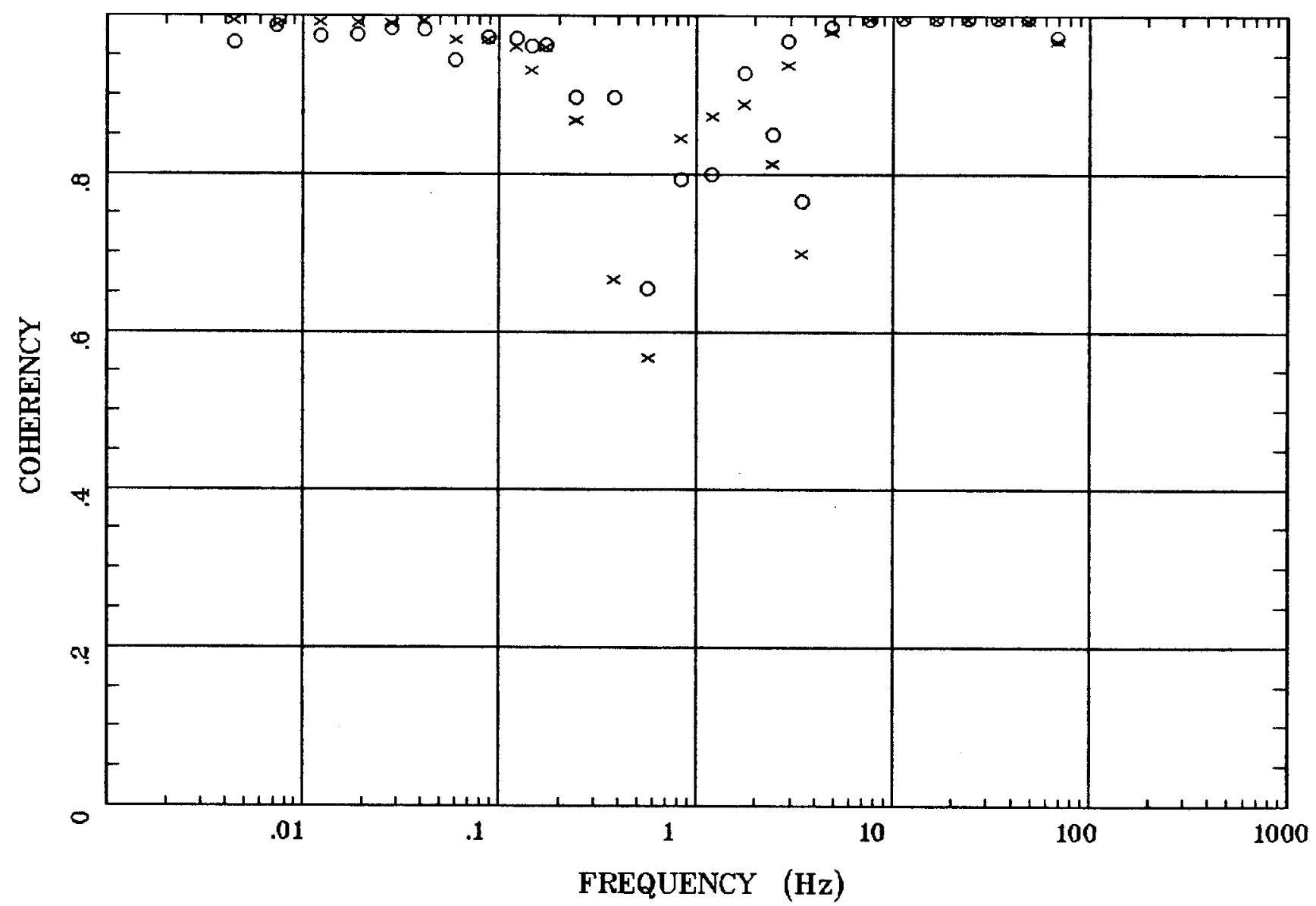

Client:

Remote: Local B

Acquired: 10:2 Jul 31, 1998

Survey Co:USGS GD-MRP Denver
Rotation:

Filename: hr35.avg

Channels: Ch1 Ch2 Ch3 Ch4 Ch5 Ch8 Ch9

Plotted: 13:44 Dec 07, 2000

< EMI - ElectroMagnetic Instruments > 
POLAR PLOTS

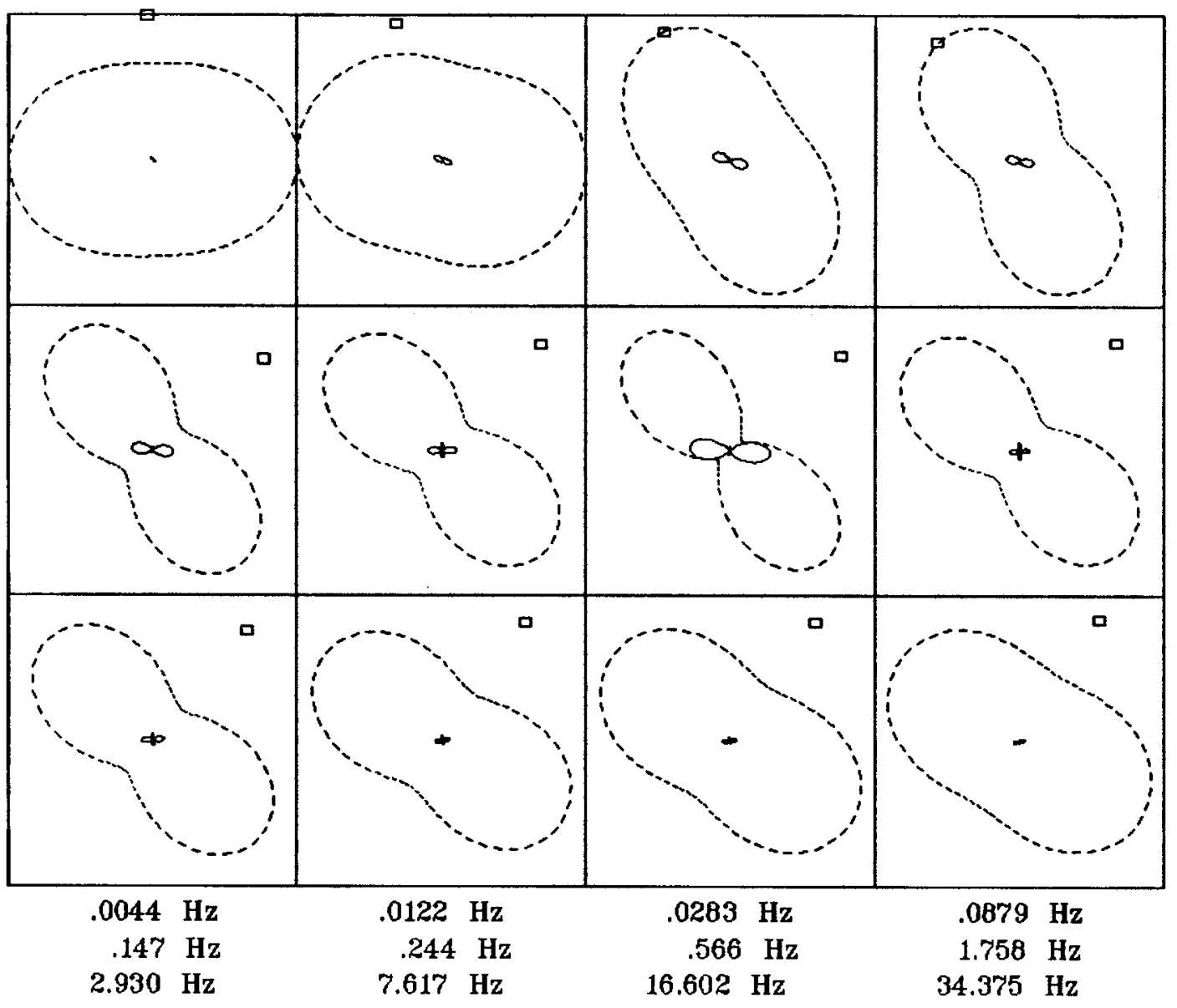

Client:

Remote: Local $B$

Acquired: 10:2 Jul 31, 1998

Survey Co:USGS GD-MRP Denver
Humboldt River Line 1

Rotation:

Filename: hr35.avg

Channels: Ch1 Ch2 Ch3 Ch4 Ch5 Ch8 Ch9

Plotted: 13:45 Dec 07, 2000

$<$ EMI - ElectroMagnetic Instruments 
APPARENT RESISTIVITY

Humboldt River Line 1

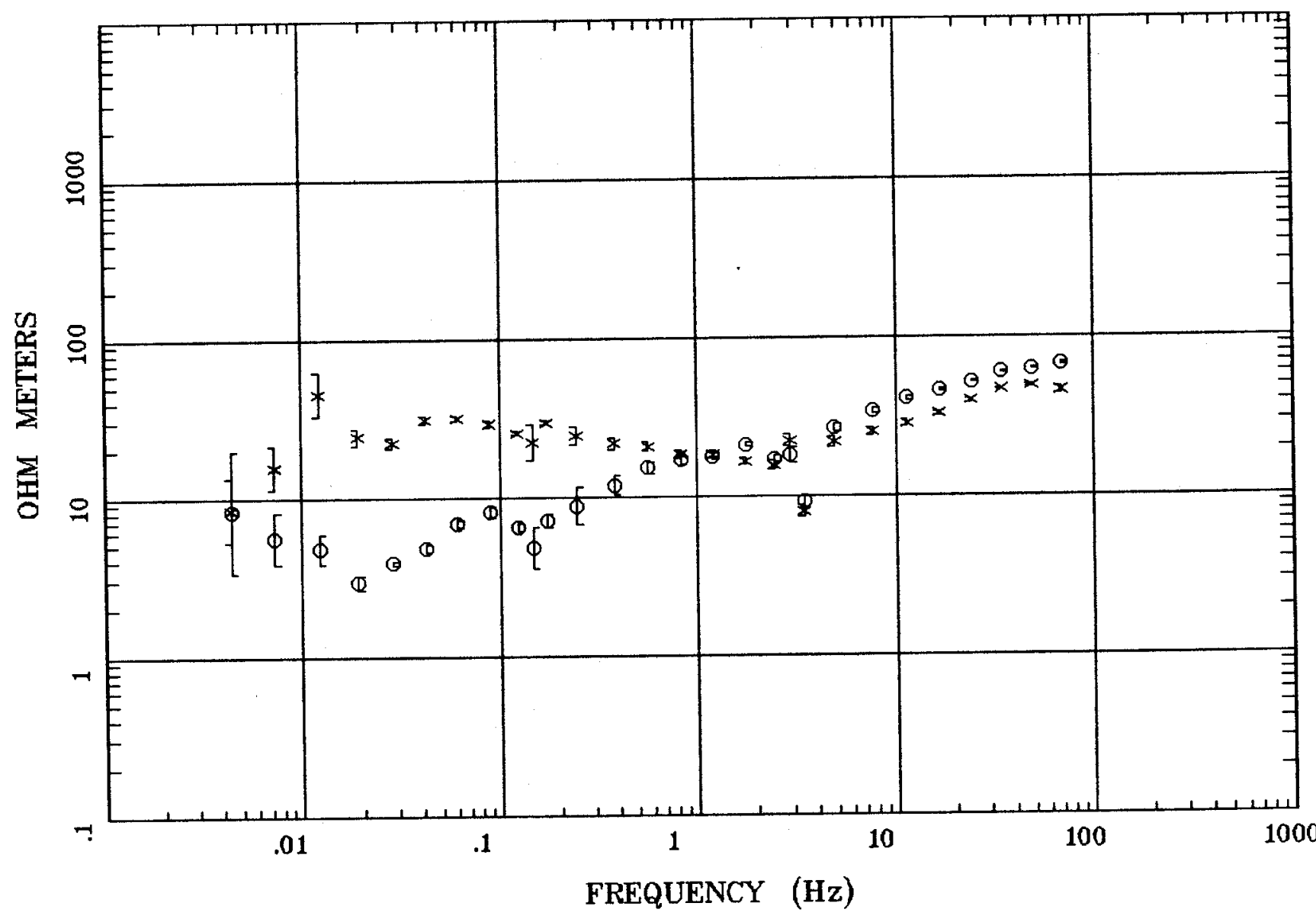

Client:

Remote: Local B

Acquired: 15:1 Jul 31, 1998 Survey Co:USGS GD-MRP Denver
Rotation:

Filename: hr56.avg

Channels: Ch1 Ch2 Ch3 Ch4 Ch5 Ch8 Ch9

Plotted: 13:56 Dec 07, 2000

$<$ EMI - ElectroMagnetic Instruments 
IMPEDANCE PHASE

Humboldt River Line 1

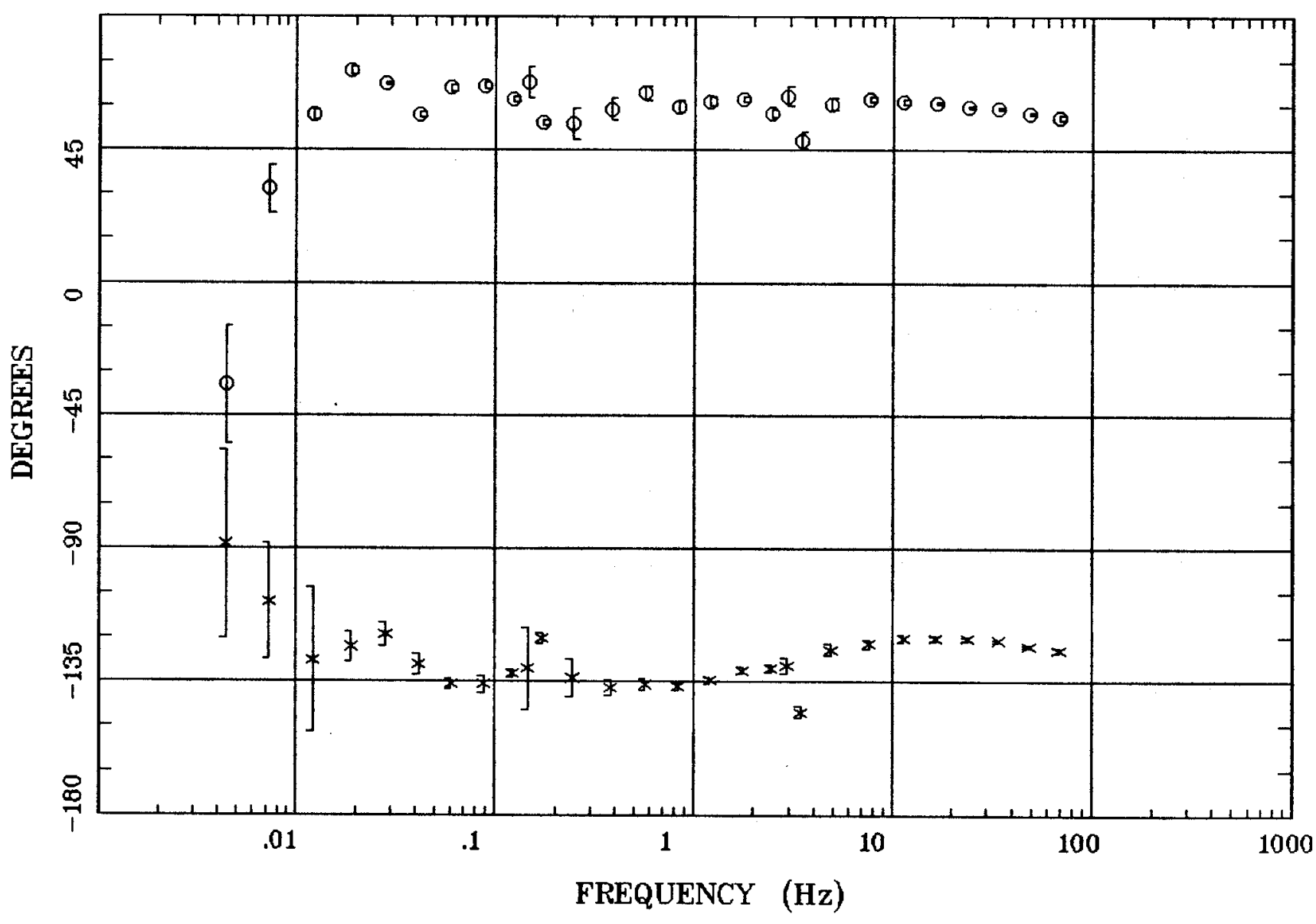

Client:

Remote: Local B

Acquired: $15: 1$ Jul 31,1998 Survey Co:USGS GD-MRP Denver
Rotation:

Filename: hr56.avg

Channels: Ch1 Ch2 Ch3 Ch4 Ch5 Ch8 Ch9

Plotted: 13:56 Dec 07, 2000

$<$ EMI - ElectroMagnetic Instruments > 


\section{ROTATION ANGLE}

Humboldt River Line 1

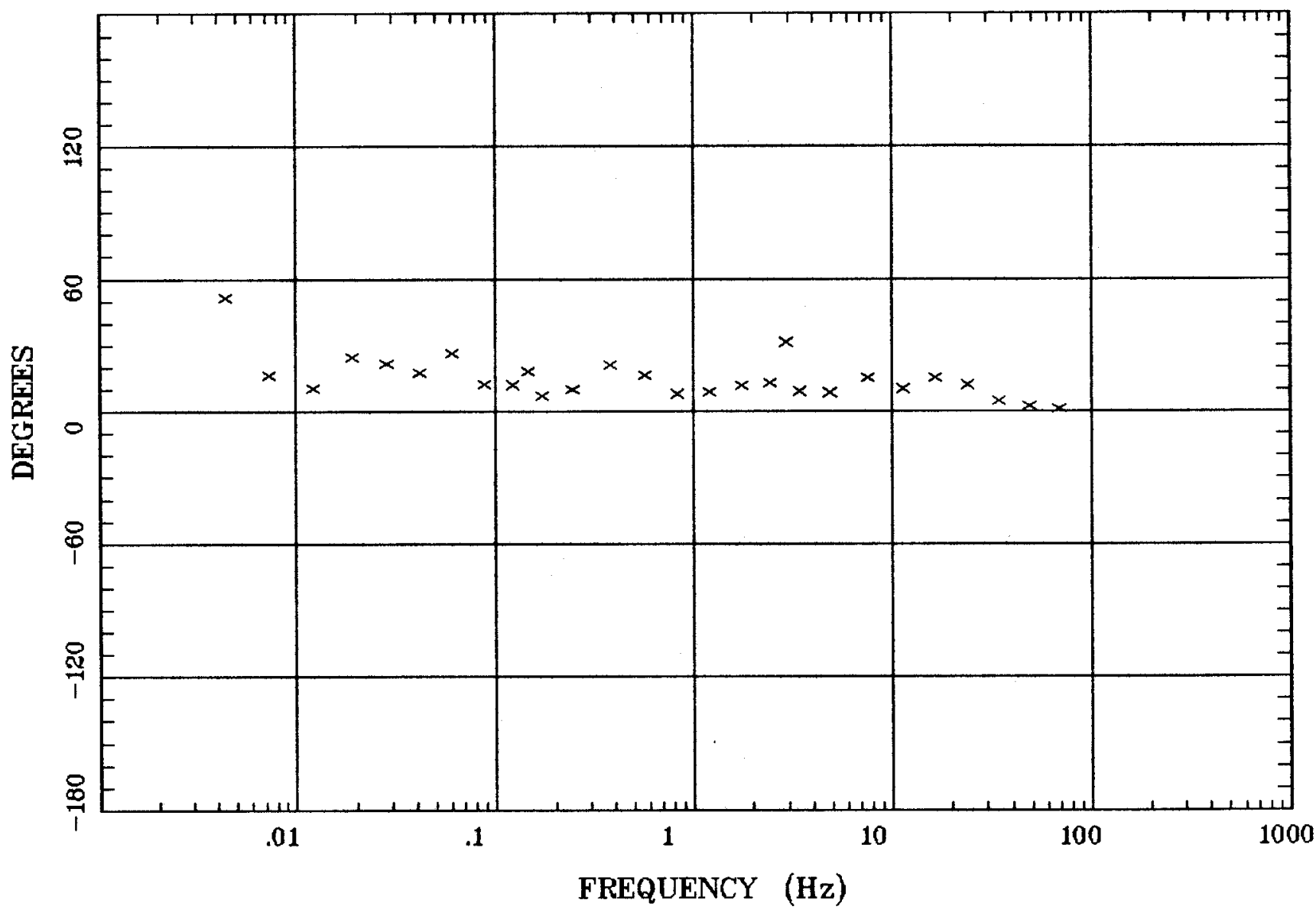

Client:

Remote: Local B

Acquired: 15:1 Jul 31, 1998

Survey Co:USGS GD-MRP Denver
Rotation:

Filename: hr56.avg

Channels: Ch1 Ch2 Ch3 Ch4 Ch5 Ch8 Ch9

Plotted: 13:56 Dec 07, 2000

< EMI - ElectroMagnetic Instruments > 


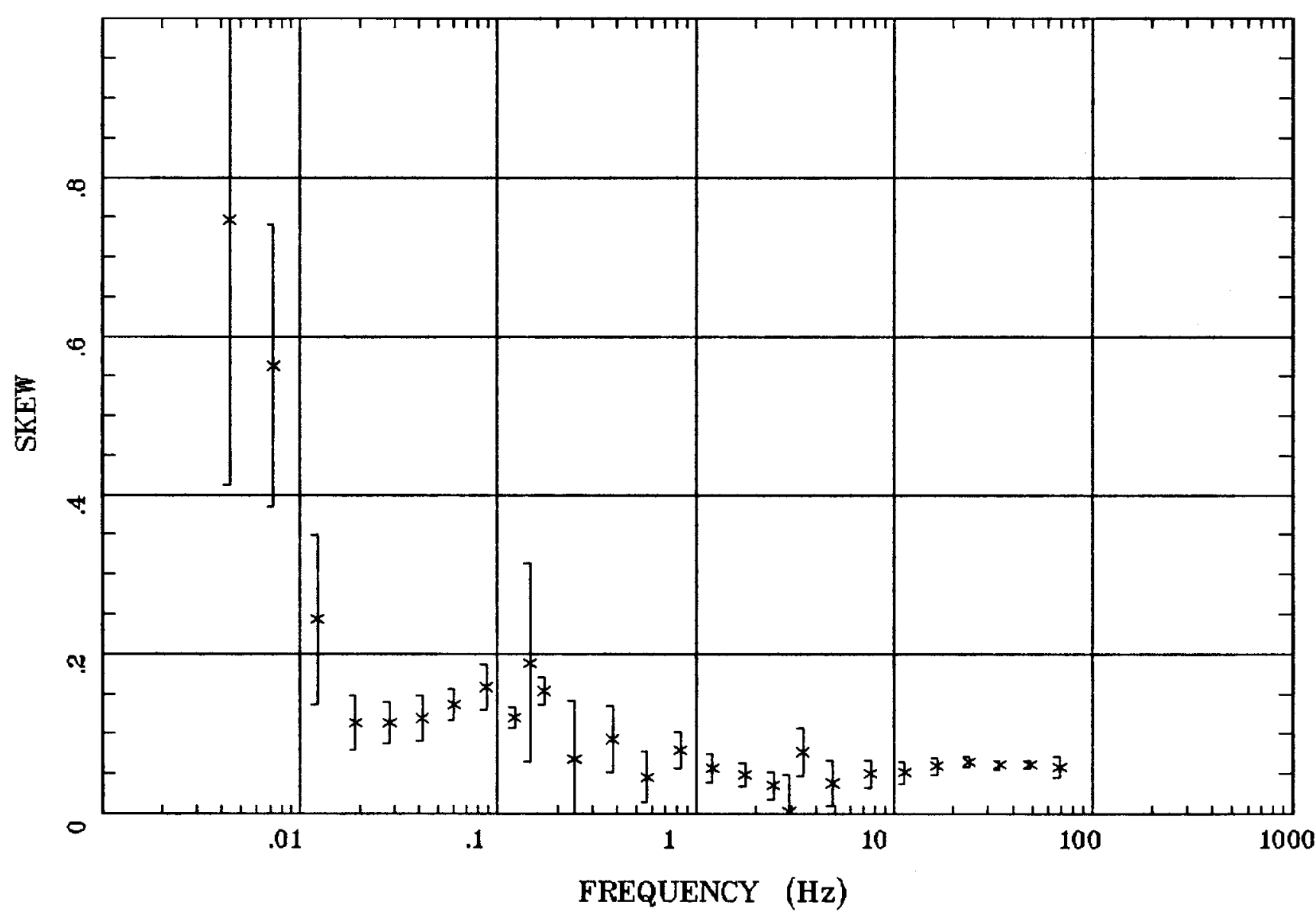

Client:

Remote: Local B

Acquired: 15:1 Jul 31, 1998 Survey Co:USGS GD-MRP Denver
Rotation:

Filename: hr56.avg

Channels: Ch1 Ch2 Ch3 Ch4 Ch5 Ch8 Ch9 Plotted: 13:56 Dec 07, 2000

< EMI - ElectroMagnetic Instruments > 


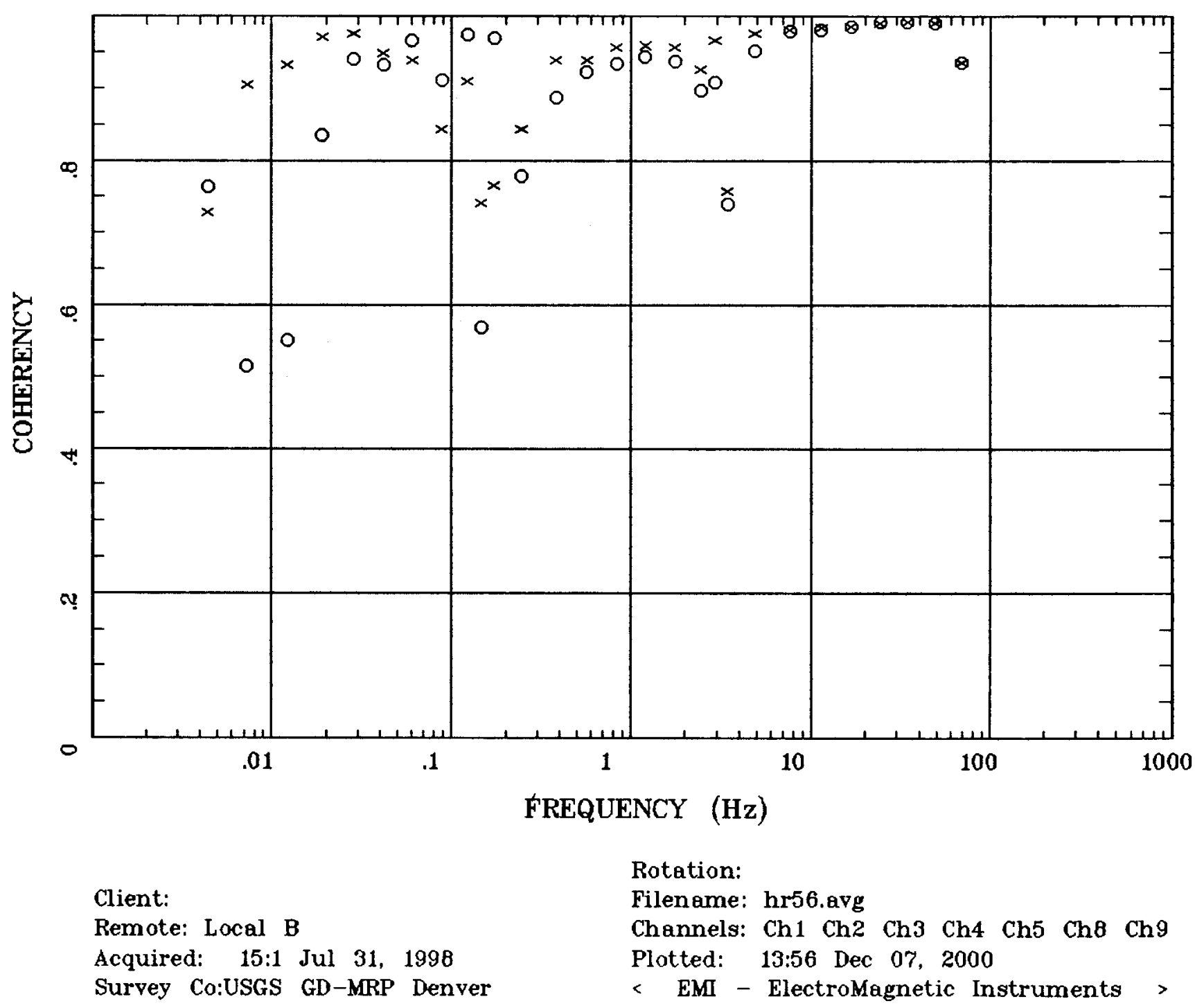


Humboldt River Line 1

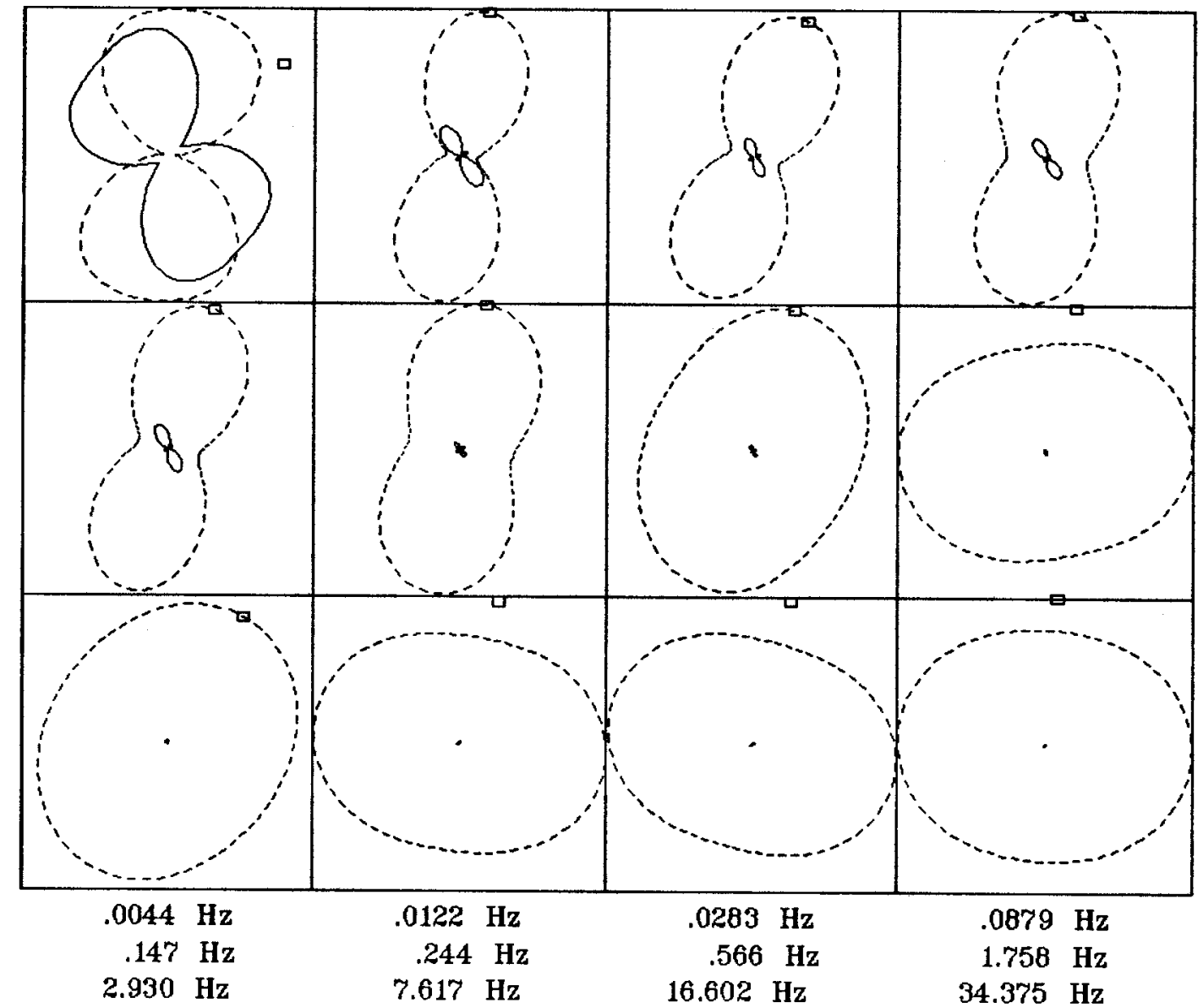

\section{Client:}

Remote: Local B

Acquired: $15: 1$ Jul 31, 1998 Survey Co:USGS GD-MRP Denver
Rotation:

Filename: hr56.avg

Channels: Ch1 Ch2 Ch3 Ch4 Ch5 Ch8 Ch9

Plotted: 13:56 Dec 07, 2000

< EMI - ElectroMagnetic Instruments 